\title{
Efficient Log Likelihood Ratio Estimation for Polar Codes
}

\author{
by \\ Alaa Abdulameer Hasan, M. Sc. \\ A dissertation submitted to the \\ Faculty of Graduate Studies and Research \\ in partial fulfillment of the requirements for the degree of
}

Doctor of Philosophy in Electrical and Computer Engineering

Ottawa-Carleton Institute for Electrical and Computer Engineering (OCIECE)

Department of Systems and Computer Engineering

Carleton University

Ottawa, Ontario

May, 2018

(C) Copyright

Alaa Abdulameer Hasan, 2018 
The undersigned hereby recommends to the Faculty of Graduate Studies and Research acceptance of the dissertation

\title{
Efficient Log Likelihood Ratio Estimation for Polar Codes
}

\author{
submitted by Alaa Abdulameer Hasan, M. Sc. \\ in partial fulfillment of the requirements for the degree of \\ Doctor of Philosophy in Electrical and Computer Engineering
}
Professor Salama Ikki, External Examiner, Department of Electrical Engineering, Lakehead University

Professor Ian D. Marsland, Thesis Supervisor

Professor Yvan Labiche, Chair,

Department of Systems and Computer Engineering

Ottawa-Carleton Institute for Electrical and Computer Engineering (OCIECE)

Department of Systems and Computer Engineering

Carleton University

May, 2018 


\section{Abstract}

Although polar codes, proposed by E. Arikan, achieve maximum capacity for binary input discrete memoryless channels (B-DMCs) and have outstanding asymptotic behaviour, the error rate performance for practical lengths is not as competitive as low-density parity-check (LDPC) or turbo codes. This thesis presents various solutions to this problem and shows that polar codes are suitable not only for channel coding but also achieve optimal performance for several other important problems in information theory.

In the first study we aim at improving the error rate performance of polar codes by applying the well known normalized minimum-sum (NMS) decoding algorithm where the messages are scaled so as to enhance the reliability of the propagated messages and to increase the decoding accuracy. Furthermore, the minimum-sum (MS) algorithm itself is modified through using a correction factor which has been preferred for many practical applications since they offer comparable decoding performance compared to that of sum-product (SP) and can recover the bit error rate (BER) loss of the MS decoding. In addition, the scaling factor of the normalization is not fixed as it depends on polar code length and rate. The optimal parameter for this factor can only be selected by simulation prior to implementation via density evolution (DE); where the threshold values are calculated for various scaling factors and the scaling factor showing the highest threshold in noise level (equivalently the lowest threshold in SNR) is determined as the optimal scaling factor.

We then study the problem of log likelihood ratio (LLR) correction in uncorrelated fading channels when the fading gain is not known at the receiver and propose a method for finding the linear scaling factors that minimize the probability of error of a maximum likelihood decoder. In order to perform LLR scaling that is adaptive to various transmission conditions, an efficient mutual information maximization based scaling factor searching algorithm is developed. Following that, a non-uniform 
quantizer based on the condition of maximum information rate achieved over uncorrelated Rayleigh fading channel and when successive cancellation (SC) decoding algorithm of polar codes is applied. With no important performance degradation, the suggested quantizer shows a considerable complexity decreasing of look-up table (LUT) realization by applying sort of squeeze method.

In the next study, we present a first-order linear LLR approximation with lowcomplexity for calculating soft metrics of polar coded quadrature amplitude modulation (QAM) system in additive white Gaussian noise (AWGN) and Rayleigh fading channels under single/multiple transmit and receive antennas. A simple bit LLR expression is provided for Gray coded QAM signals and realized to approximate the complicated theoretical LLR functions. To minimize the complexity of the max-logMAP algorithm, LLRs can be simplified by changing the mathematical minimum functions with simple linear functions. This is achieved by averaging the LLR values in different regions in order to get a single simplified expression.

Lastly, we examine the use of the SC polar coded QAM constellation to approach the capacity of wireless Rayleigh fading channels over multiple transmit and receive antennas when ideal channel state information is available at both receiver and transmitter. We put some necessary conditions at the transition points that make the optimal distribution determination simple. Specifically, by using Hermite polynomials and under an even-moment constraint, the results show that the information rate is achieved with unique and optimal input distribution. The computational complexity can be reduced by factorizing the optimal distribution into the product of symmetrical distributions. 
"O people! We have created you from a male and a female and made you into nations and tribes that you may know one another."

The Holy Qur'an, Chapter 49, Verses 13 
Dedicated to my wife, Hiba Sahib Mahdi 


\section{Acknowledgments}

First, I thank God, the Gracious and the Merciful, for His countless blessings.

I am truly honored and fortunate to have been advised by Prof. Ian D. Marsland. This thesis would not have been possible without his guidance and support. He has been a constant source of inspiration, knowledge, and creativity. I feel I am one of the few lucky persons whose supervisor is a guide and teacher put together. His doors were always open for discussions which range from coding theory to programming tactics. I would also like to acknowledge his efforts to improve my writing skills which were non-existent before. For all the above and many other reasons I would like to express my deepest gratitude and appreciation to him.

I would like to thank my thesis committee members Prof. Claude D'Amours, Prof. Halim Yanikomeroglu, Prof. Calvin Plett, and Prof. Salama Ikki for their very helpful comments and suggestions. My sincere respects goes to Prof. Yanikomeroglu for his generous support, kindness, and advice.

It was a great pleasure to study at the University of Carleton. I have enjoyed my time spent here, both within and outside my research. I am also very grateful to my awesome lab mates, Yoones Hashemi, Peyman Neshaastegaran, Monirosharieh Vameghestahbanati, and Mohamed Alzenad. They helped me in the hours of distress and made my journey through $\mathrm{PhD}$ a lot easier. Talking to them was always refreshing, and their continuous encouragement kept me focused on my goals.

My PhD study was mostly sponsored by the Iraqi Government through the ministry of higher education and scientific research (MOHESR). I thankfully acknowledge this support.

My words cannot express my gratitude toward my parents who, despite all hardships, provided me with everything I ever needed. At times, the prayers of my parents were the only thing to give me the confidence to overcome my problems. Being away from my parents was the biggest sacrifice I made for graduate studies. In addition to my parents, my sister and my brothers supported me throughout my life. At the end 
I would like to express my heartfelt thanks to my beautiful children as well as my wonderful and beloved wife, Hiba, who carried the burden during the last years and was always my support in moments of doubt and fatigue. Thank you, my family. 


\section{Table of Contents}

Abstract $\quad$ iii

Acknowledgments vii

Table of Contents $\quad$ ix

List of Tables $\quad$ xii

List of Figures $\quad$ xiii

Nomenclature $\quad$ xvi

1 Introduction 1

1.1 Polar Codes . . . . . . . . . . . . . . . . . . . . 1

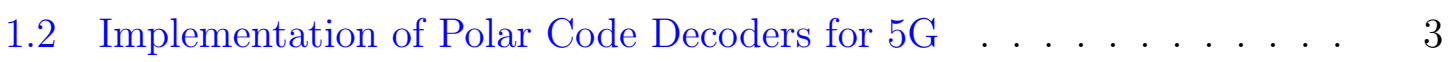

1.3 Summary of Contributions . . . . . . . . . . . . . . 4

1.4 Related Publications . . . . . . . . . . . . . . . . . . 6

1.5 Organization of the Thesis . . . . . . . . . . . . . 7

2 Preliminaries $\quad 8$

2.1 Channel Model . . . . . . . . . . . . . . . . . . . 8

2.2 Block Codes . . . . . . . . . . . . . . . . . . 9

2.2.1 Single Parity-Check Codes . . . . . . . . . . . . . . . 10

2.3 Review of Polar Codes . . . . . . . . . . . . . . . . . . . 13

2.3.1 Channel Combining . . . . . . . . . . . . . . . . . 14

2.3.2 Channel Splitting . . . . . . . . . . . . . . . 17

2.3.3 Recursive Channel Transformation . . . . . . . . . . . . . . . 17

2.4 Phenomenon of Channel Polarization . . . . . . . . . . . . . 18 
2.5 Polar Codes Encoding . . . . . . . . . . . . . . . . . . . . 20

2.6 Successive Cancellation Decoding of Polar Codes . . . . . . . . . . . 20

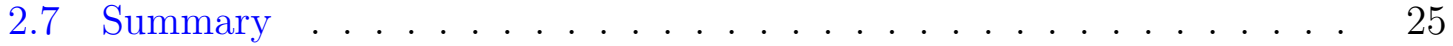

3 Corrected NMS SC Decoding of Polar Codes 26

3.1 Reduced-Complexity Check Node Calculations . . . . . . . . . 28

3.1 .1 Minimum-Sum (MS) Algorithm . . . . . . . . . . . . . . 31

3.1.2 Normalized Minimum-Sum (NMS) Algorithm . . . . . . . . 32

3.1.3 Corrected Minimum-Sum Algorithm . . . . . . . . . . . 32

3.2 Corrected Normalized Minimum-Sum Algorithm . . . . . . . . . . . 33

3.2.1 Normalization Term Calculation Using Rate of Means . . . . . 34

3.2 .2 Complexity Discussion . . . . . . . . . . . . . . . . . 42

3.3 Performance of NMS-SC Polar Decoding Under Density Evolution Method . . . . . . . . . . . . . . . . . 42

3.3.1 Justification of Gaussian Approximation . . . . . . . . . . 43

3.3 .2 Alternative Proof . . . . . . . . . . . . . . . . . . 45

3.3.3 Channel Symmetry Condition . . . . . . . . . . . . . . 47

3.3.4 Optimization Factor, $\boldsymbol{c} \ldots \ldots \ldots \ldots \ldots$

3.4 Numerical Results . . . . . . . . . . . . . . . . . . . . . . . 47

3.5 Summary . . . . . . . . . . . . . . . . . . . . . . . . . . 49

4 Non-Uniform Quantizers with SC Polar Based Channel-Optimized Decoders $\quad 51$

4.1 Low Complexity LLR Calculation Without Channel State Information 52

4.1 .1 System Model . . . . . . . . . . . . . . . . . . . . . 52

4.1 .2 Linear LLR Approximation . . . . . . . . . . . . . 54

4.1.3 Achievable Rates under Unknown CSI . . . . . . . . . . . 54

4.1.4 Optimization of the Scaling Factor . . . . . . . . . . 55

4.2 Non-Uniform Quantization for SC Polar codes . . . . . . . . . . 58

4.2.1 Effects of Quantization on Channel Capacity . . . . . . . . 58

4.2.2 Non-Uniform Quantization Scheme . . . . . . . . . . . . 62

4.3 Numerical Results . . . . . . . . . . . . . . . . . . . . . . . 65

4.4 Summary . . . . . . . . . . . . . . . . . . 65 
5 Low Computational Complexity of SC Polar Decoder in MIMO $\begin{array}{ll}\text { Fading Channel } & 67\end{array}$

5.1 System Model . . . . . . . . . . . . . . . . . . . . . . 68

5.2 The Problem . . . . . . . . . . . . . . . . . . 70

5.2.1 Capacity-Achieving Distribution Characterization . . . . . 71

5.2 .2 The Optimal Input . . . . . . . . . . . . . . . . . . 73

5.3 Capacity Under Amplitude-Limited Inputs . . . . . . . . . . . 77

5.4 Factorizing the Optimal QAM Distribution . . . . . . . . . . 80

5.5 Numerical Results . . . . . . . . . . . . . . . . . . . . . . . . . . 82

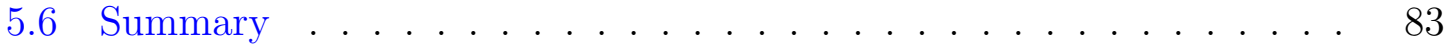

6 Conclusions and Future Work $\quad 84$

$\begin{array}{ll}\text { List of References } & 94\end{array}$

Appendix A Low Computational Complexity LLR Metrics for Polar $\begin{array}{ll}\text { Coded QAM } & 95\end{array}$

A.1 System Model . . . . . . . . . . . . . . . . . . . . 95

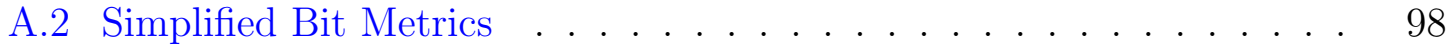

A.3 LLR in Transmit Diversity . . . . . . . . . . . . . . . . 102

A.4 Numerical Results. . . . . . . . . . . . . . . . . . . . 103

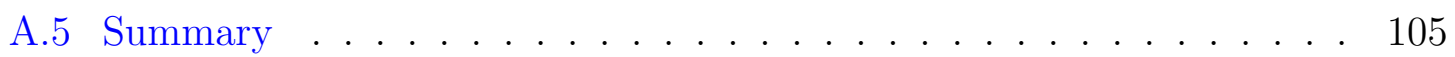

$\begin{array}{ll}\text { Appendix B Proof and Derivation } & 106\end{array}$

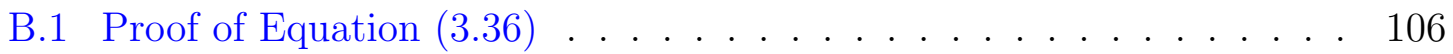

B.2 Derivation of Equation (3.51) $\ldots \ldots \ldots \ldots \ldots \ldots$

B.3 Tangent Line Approximation . . . . . . . . . . . . . . . 117

Appendix C Hermite Polynomials $\mathbf{H}_{n}(x)$ : Some Basics 120 


\section{List of Tables}

3.1 Threshold values for polar code of length 4096 and rate 0.5 with two normalization factor $c$. . . . . . . . . . . . . . . . . 49

4.1 Uniform and non-uniform quantization methods with $\mathrm{q}=6$ and $\mathrm{f}=3 \quad 64$

A.1 LLRs calculations complexity in terms of complex operations. $\mathrm{O}$ is Original and $\mathrm{S}$ is Simplified. . . . . . . . . . . . . . . . . 103 


\section{List of Figures}

1.1 Mutual information of bit channels, $N=1024$. . . . . . . . . . . . 2

1.2 Mutual information of bit channels, $N=32768$. . . . . . . . . . 3

2.1 Essential communication system units. . . . . . . . . . . . . . 8

2.2 Block coding. . . . . . . . . . . . . . . . . . . . . . . . . 10

2.3 Message passing representation. . . . . . . . . . . . . . . . 13

2.4 Left figure: the BEC with erasure probability z. An input symbol to this channel is erased with probability $z$ or passed through the channel with probability $1-z$. Middle figure: the BSC with cross-over probability $\epsilon$. An input to this channel is flipped with probability $\epsilon$ or passed through with probability $1-\epsilon$. Right figure: the BAWGN with noise variance $\sigma^{2}$. The output $y$ of this channel is the sum of its input $x \in\{0,1\}$ and a noise value $n$ which is a Gaussian random variable with mean 0 and variance $\sigma^{2}$. . . . . . . . . . . . . . . . 14

2.5 Channels a) $\boldsymbol{W}_{2}$, b) $\boldsymbol{W}_{4}$.................. 15

2.6 Channel $\boldsymbol{W}_{N} \ldots \ldots \ldots \ldots \ldots$

2.7 Channel polarization examples probability $\frac{1}{2}$ a) $N=2^{0}$; b) $N=2^{1}$, and c) $N=2^{2}$. . . . . . . . . . . . . . . . . . . . . . . . . . . 19

2.8 Encoding algorithm for an $(8,4)$ polar code. . . . . . . . . . . . 21

2.9 Trellis representations of polar codes. . . . . . . . . . . . . . . . 22

2.10 Polar encoder with $N=8$ and $R=0.5 \ldots \ldots . \ldots . . \ldots 24$

2.11 SC polar decoder with $N=8$ and $R=0.5 \ldots \ldots . . . . .25$

3.1 LLR decisions. . . . . . . . . . . . . . . . . . . . . . . . . . 30

3.2 BLER performances for decoding algorithms assuming $N=1024 \mathrm{SC}$ polar code with code rate $R=0.5$ and $c=0.869$. . . . . . . . . 41

3.3 SP/NMS SC decoder performances under different blocklengths with $R=0.5$. . . . . . . . . . . . . . . . . . . . . . 42

3.4 Density function before and after normalization with $c=0.787$. . . 48 
3.5 Effect of $c$ on BLER for $N=4096$ blocklength polar code decoded by NMS-SC.

3.6 Error rates with $N=4096$ and half rate polar code under two different decoding algorithms. . . . . . . . . . . . 50

4.1 The achievable information rate under multiple values of $\sigma \ldots \ldots 58$

4.2 C under true LLR compared with $\widehat{\mathrm{C}}$ under $\hat{\alpha}_{\text {opt }}=0.627 \ldots \ldots . . . \quad 59$

4.3 True LLR compared to two approximated LLRs under $\sigma=0.65$, LLR with $\hat{\alpha}=\mathrm{E}[\alpha]=\frac{\sqrt{\pi}}{2}$ and LLR with $\hat{\alpha}_{\mathrm{opt}}=0.627 \ldots \ldots \ldots$

4.4 BLER under different LLR calculation for SC polar code length of 1024

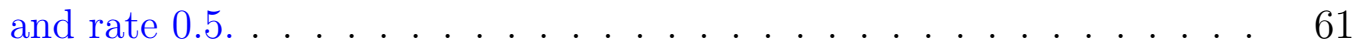

4.5 The quantization scheme. . . . . . . . . . . . . . . . . 61

4.6 Capacity for (un)quantized channel without CSI measurements based on LLR optimized linear approximation with $\hat{\alpha}_{\text {opt }}=0.627 \ldots \ldots .62$

4.7 Simulated BLER for the length 1024 and rate 0.5 SC polar codes with different decoding scenarios. . . . . . . . . . . . . . 63

4.8 The nonlinear function $f(x)=\ln \frac{e^{x}+1}{e^{x}-1}, x>0 \ldots \ldots \ldots \ldots 6$

4.9 Simulated BLER for the length 1024 and rate $0.5 \mathrm{SC}$ polar codes with different decoding scenarios. NUQ stands for non-uniform quantization. 66

5.1 Signal constellation of $\mathcal{X}_{16-\mathrm{QAM}} \ldots \ldots \ldots \ldots \ldots \ldots$

5.2 Achieved capacities under different transmission scenarios. The SC polar codes has code length of 1024 with half rate. . . . . . . . . . 82

5.3 Achieved capacities under different transmission scenarios. The SC polar codes has code length of 1024 with half rate. . . . . . . . . 83

A.1 Detection regions for 16-QAM constellation. . . . . . . . . . 96

A.2 Exact and approximated (piece-wise linear and by averaging and differentiation) LLR $\left(b_{1}\right)$ for 16-QAM with $|h|=1$ and $\sigma=0.5 \ldots \ldots .100$

A.3 Exact and approximated (piece-wise linear and by differentiation) $\operatorname{LLR}\left(b_{1}\right)$ for $64-$ QAM with $|h|=1$ and $\sigma=0.5 \ldots \ldots \ldots 10 \ldots$

A.4 BER performance of uncoded 16/64-QAM with/without approximation and with/without transmit diversity. . . . . . . . . . . 104

A.5 BER performance of SC polar coded 16-QAM with/without approximation. $R=0.5$ and $N=1024$. . . . . . . . . . . . . 105

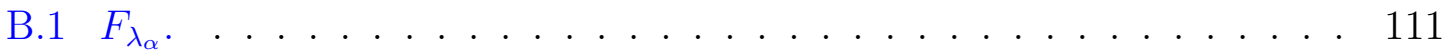

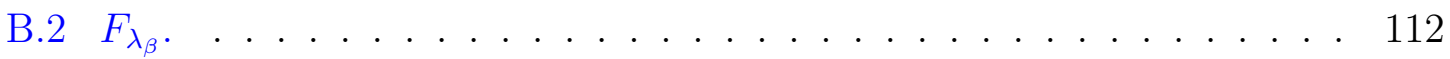




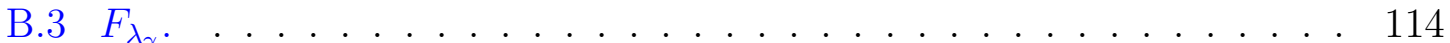

B.4 $\operatorname{Pr}\left\{\lambda_{0}>\frac{-\ell}{2 \lambda_{1}}, \lambda_{0} \geqslant \lambda_{1}\right\}+\operatorname{Pr}\left\{\lambda_{1}>\frac{-\ell}{2 \lambda_{0}}, \lambda_{1} \geqslant \lambda_{0}\right\} \simeq \frac{1}{2} \ldots \ldots .116$

B.5 Tangent as a linear approximation to a curve. . . . . . . . . . 119 


\title{
Nomenclature
}

\author{
B-DMC Binary input Discrete Memoryless Channel \\ MS Minimum-Sum \\ NMS Normalized Minimum-Sum \\ SP Sum-Product \\ DE Density Evolution \\ LDPC Low-Density Parity-Check \\ BER Bit Error Rate \\ BLER Block Error Rate \\ LLR Log Likelihood Ratio \\ SNR Signal-to-Noise Ratio \\ SC Successive Cancellation \\ SCL Successive Cancellation List \\ LUT Look-Up Table \\ QAM Quadrature-Amplitude-Modulation \\ AWGN Additive White Gaussian Noise \\ CSI Channel State Information \\ PAM Pulse-Amplitude-Modulation \\ MIMO Multiple-Input Multiple-Output
}


BP Belief Propagation

BPSK Binary Phase Shift Keying

NUQ Non-Uniform Quantization

CDF Cumulative Distribution Function

PDF Probability Density Function

MGF Moment Generating Function

i.i.d. Independent and Identically Distributed

i.u.d. Independent and Uniformly Distributed 


\section{Chapter 1}

\section{Introduction}

In real world communication systems, where the transmitted signal is corrupted by channel noise, it is useful for redundancy to be added to the transmitted signal to assist the receiver in recovering the transmitted data. This procedure is known as the channel coding.

\section{$1.1 \quad$ Polar Codes}

While turbo and LDPC codes have brought capacity-approaching performance within reach of implementable systems, implementable does not necessarily mean practical. The complexity of codes that perform well under practical constraints such as limited decoding delay and high spectral efficiency is still a major hurdle for low power implementations in integrated circuits. There is a serious need for new methods that simplify code design, construction, storage, and decoder implementation.

Polar codes, proposed by E. Arikan [1], are provably able to achieve maximum capacity under B-DMCs with low encoding/decoding computation of $\mathrm{O}\left(\mathrm{N} \mathrm{log}_{2} \mathrm{~N}\right)(\mathrm{N}$ is the code length). The capacity of the channel created between input and output bits polarizes to zero or to one. The analysis and construction of polar codes are summarized as follows: (1) Given a symmetric B-DMC, channels between the bits at the input of a linear encoder and the channel output sequence are created, such that the capacity of these channels polarizes to either zero or one; the proportion of such channels with capacity close to one converges to the capacity of the original channel. This phenomenon is termed as channel polarization. (2) By transmitting data bits through the noiseless channels, polar codes achieve the capacity under successive cancellation (SC) decoding. Fig. 1.1 and Fig. 1.2 show the mutual information as the 


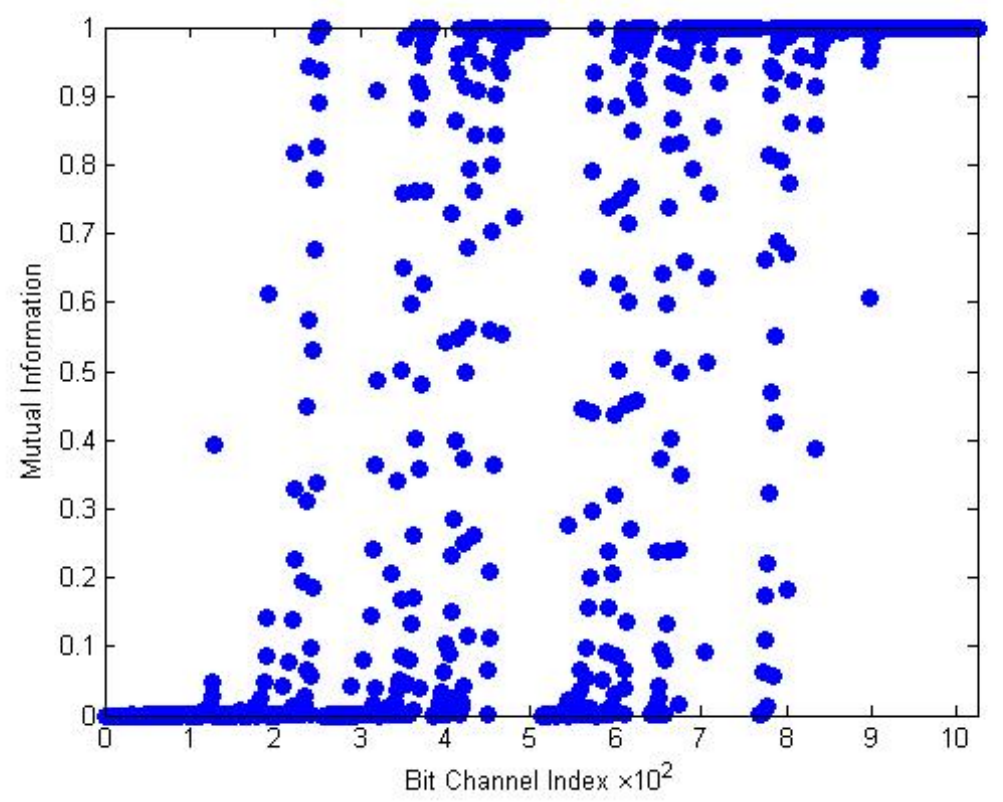

Figure 1.1: Mutual information of bit channels, $N=1024$.

blocklength increases.

In his work, the Arikan's input transformation matrix is $\mathbf{G}_{2}^{\otimes n}$ where $\mathbf{G}_{2}=\left[\begin{array}{ll}1 & 0 \\ 1 & 1\end{array}\right]$, and $\otimes n$ denotes the $n$th Kronecker product. Polar codes are presented so that some bits are chosen as data bits whereas the others are settled to nominate $\mathbf{G}_{2}^{\otimes n}$ rows. In Reed-Muller codes [2,3], these rows are chosen so that the minimum distance of the code is maximized; while in polar codes those rows are picked to optimize the achievement under the successive cancellation (SC) decoding algorithm.

Polar codes were initially introduced with low complexity using the successive cancellation (SC) algorithm [1]. As a generalization of the classic SC decoder, the successive cancellation list (SCL) decoder for polar codes is proposed in [4], where $L$ decoding paths are considered concurrently at each decoding stage. The soft cancellation (SCAN) decoder achieves better performance than SC one at the expense of increased complexity [30]. A cyclic-redundancy check (CRC) is constructed as an outer code for concatenating to the polar code. This increases neither the computational complexity of the encoder nor that of the decoder by a notable amount, while reducing the block-error probability significantly, making the error-rate performance of the modified polar codes under SCL decoding comparable to the state-of-the-art 


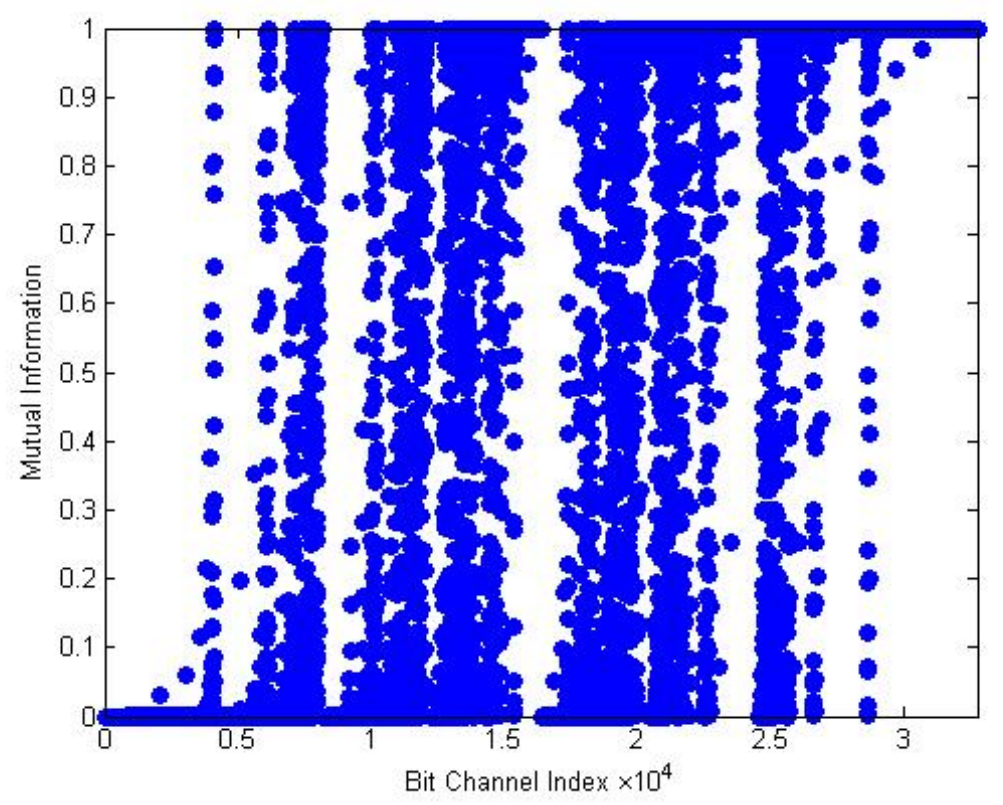

Figure 1.2: Mutual information of bit channels, $N=32768$.

LDPC codes [6].

In order to reduce latency and increase throughput, Simplified SCL (SSCL) $[7,8]$ and Fast-SSCL decoding algorithms were proposed, that guarantee significant reduction in the number of required time-steps with respect to SCL without relying on approximations or code specific design. The partitioned SCL (PSCL) algorithm in $[9,10]$ breaks the code into constituent codes (partitions) and each constituent code is decoded with the SCL algorithm.

\subsection{Implementation of Polar Code Decoders for $5 \mathrm{G}$}

Since their invention by Arikan, polar codes have been well investigated in the literature. Recent studies [11-13] have demonstrated the superior performance of polar codes compared to low-density parity-check (LDPC) and turbo codes in the context of $5 \mathrm{G}$ test scenarios.

The $5 \mathrm{G}$ standardization process is putting a particular focus on improved errorcorrection performance, lower power consumption and higher throughput. SCL-based 
decoders are currently one of the best candidates to meet $5 \mathrm{G}$ error-correction performance requirements and throughput. An FPGA implementation of SC-based decoding algorithm was able to achieve an information throughput of 1 Gbps at a clock frequency of $106 \mathrm{MHz}$ [10,14]. A double-column 1024-parallel architecture of belief propagation (BP) polar decoder enables a 4.68 Gbps throughput at a clock frequency of $300 \mathrm{MHz}$ [15]. However, some new proposed decoder implementations have been showed how decoding performance, throughput and decoder implementation correlate for a range of applicative parameters, software optimizations and hardware architectures $[16,17]$. Near future applications are based on machine-to-machine (M2M) communications that demand lower latencies than humancentric communications. For this purpose, one of the investigations is to decrease the average detection latency of a multicarrier communication system so that higher frequency diversity gain can be achieved [18].

Furthermore, the demand for various accepted cases in 5G like enhanced mobile broadband (eMBB), massive internet of things (IoT) and ultra reliable and low latency communications (URLCC) increases, thus reliable and efficient encoding and decoding methods need to be designed [19].

Polar codes have been introduced to support increased channel capacity and improved bit error rate and can be constructed/decoded using simple algorithms that are more computationally efficient than turbo and LDPC codecs, which makes them suitable for a wide range of applications.

\subsection{Summary of Contributions}

\section{Corrected NMS-SC Polar Decoders}

This work aims at improving the bit error rate (BER) performance of polar codes by applying normalized minimum-sum (NMS) decoding which is a modified version of the sum-product (SP) algorithm. The optimal parameter for the normalization factor is selected by simulation prior to implementation via density evolution (DE). This factor is calculated as a ratio of the expected value of log-likelihood ratios (LLR) and it is found that the performance of the minimum-sum (MS) algorithm could approach that of the SP algorithm. The threshold values then are calculated for various scaling factors, and the scaling factor showing the highest threshold in noise level (equivalently the lowest threshold in signal-to-noise ratio, SNR) is determined 
as the optimal scaling factor. At the same time, the maximum achievable scaling gain is estimated by the difference of threshold values.

\section{Non-Uniform Quantizers with SC Polar Based Channel- Optimized Decoders}

In this work we present a non-uniform quantizer based on the condition of maximum information rate achieved over uncorrelated Rayleigh fading channel and when the $\mathrm{SC}$ decoding algorithm is applied. Based on symmetry condition of random variables, we start with theoretical model where uniform scaling factors are applied to correct soft messages at the absence of channel state information (CSI) at the receiver side. The searching method followed to find the required optimal factor stayed away from using exhaustive search methods.

\section{Low Computational Complexity LLR Metrics for Polar Coded QAM}

An approach for calculating soft metrics of a polar coded quadrature amplitude modulation (QAM) system is presented in this study. The computational complexity of the max-log-MAP algorithm could be decreased by exchanging mathematical minimum functions of LLR expressions with new more simplified functions. We present supplementary simplification by averaging the LLR values in different regions to get a single approximate expression.

\section{Low Computational Complexity of SC Polar Decoder in MIMO Fading Channel}

Motivated by large capacity gains in multiple antenna systems when ideal CSI is available at both receiver and transmitter and QAM modulation is applied, we examine the achievable rates of Rayleigh fading channel measurement based optimization techniques. We consider a complex Gaussian noise distribution and try to determine the optimal input distribution of fixed signaling points with the aid of using Hermite polynomials and under an even-moment constraint. The computational complexity can be reduced by factorizing the optimal distribution into the product of symmetrical distributions. 


\subsection{Related Publications}

Our main contributions in this thesis, which are published or submitted for publication in [20-29] can be summarized as follows:

- [20-22] We have concentrated on the decoding of polar codes. We have shown by incorporating a piecewise linear correcting term to the LLR results through the SC polar decoder process, then the NMS algorithm performance can approach the SP decoder but there is still a noticeable degradation in performance for codes with moderately long blocklength. For this, we adopted the Gaussian approximation by means of DE which can help in obtaining the optimal parameters faster and easier, and accordingly get a very close performance to the SP decoding algorithm. This can help more for hardware realizations.

- [23-25] We have proposed a method to find the linear correction factor of the approximate LLRs for decoding SC polar codes over unknown fading channels and unavailable fading gain. Hoping to maximize the channel capacity, we proved that this approximation is fulfilled the consistency condition and maximized only with true LLRs so that it works as a measure for LLR reliability. We then suggested a quantization method for floating point on the above algorithm and proposed a non-uniform quantization scheme. From the numerical results the suggested realization for polar decoder showed $50 \%$ lowering in hardware realization with no considerable loss in performance as compared with uniform quantized decoders.

- [26-28] An optimization method is introduced in this work for approaching the capacity of fading channels with multi-antennas. Under 16-QAM modulation with the condition $\mathrm{E}\left[x^{H} x\right] \leqslant P$ for some $P>0$ and the aid of Hermit polynomials, an optimum Gaussian distribution with its correction factor are determined. Moreover, we have proved that the complexity of the proposed algorithm could be much reduced by factoring the determined Gaussian distribution into a couple of 4-PAM signals.

- [29] We analyzed the BER performance of 16/64-QAM schemes in AWGN and Rayleigh fading channels without and with transmit diversity. This analysis extends to the use of SC polar coded 16-QAM scheme, and both of BER performances are simulated with exact and approximated LLRs calculations. It 
is showed that for 16-QAM system the new simplified metrics have the same performance as the original ones while the complexity of a polar decoder can be significantly lowered without compromising the performance. The 64-QAM scheme exhibited a noticeable degradation.

\subsection{Organization of the Thesis}

The rest of this thesis is organized as follows. Basic background is provided in Chapter 2. In Chapter 3, we explore the principle of normalized minimum-sum SC decoding of polar codes. Via DE, parameters for NMS algorithm can be properly chosen to achieve very close performance to that of SP algorithm. As well, the complexity reduction is realized by using linear functions instead of nonlinear ones. In Chapter 4, we propose a methodology for correcting the soft information exchanged in the process without the presence of CSI at the receiver. Uniform scaling factors are selected so as to the achieved information rate approaches the channel capacity. This methodology helps us to minimize the number of quantization bits and, accordingly, design a non-uniform quantizer in significantly reduced hardware form. In Chapter 5, we present a numerical method to compute the capacity capacity-achieving distribution of the power constrained memoryless multiple-input multiple-output (MIMO) fading channel with a finite input constellation. An algorithm was proposed to compute the optimal input distribution as well as the optimal scaling parameter of the amplifier in the transmitter. By using Hermite polynomials and under an even-moment constraint $\mathrm{E}\left[x^{H} x\right] \leqslant P$ for some $P>0$, the optimal input distribution is only Gaussian and continuous. Some ideas for future work are presented in Chapter 6 and, finally in Appendix A, a linear approximation for calculating soft decision metrics of polar coded QAM is presented. We introduce a suboptimality by averaging over the possible linear pieces which could reduce the complexity in terms of complex operations needed for each bit metric. 


\section{Chapter 2}

\section{Preliminaries}

An essential background is presented in this chapter. First we introduce the channel transformation and how virtual channels are generated. We then describe the phenomenon of channel polarization, and lastly explain numerically the encoding scheme as well as the SC decoding scheme of polar codes.

\subsection{Channel Model}

In Fig. 2.1, let $m$ be the transmitted message, $\mathbf{x}$ is the encoded sequence, and $\mathbf{y}$ be the observed sequence. For a given $\mathbf{y}$ there is decoded version of $m, \hat{m}$, which is an estimation of the transmitted data. Throughout this thesis, we will focus on discrete memoryless channels (DMCs). Let $\operatorname{Pr}\{\mathbf{y} \mid \mathbf{x}\}$ be the channel transition probabilities. We can define the following

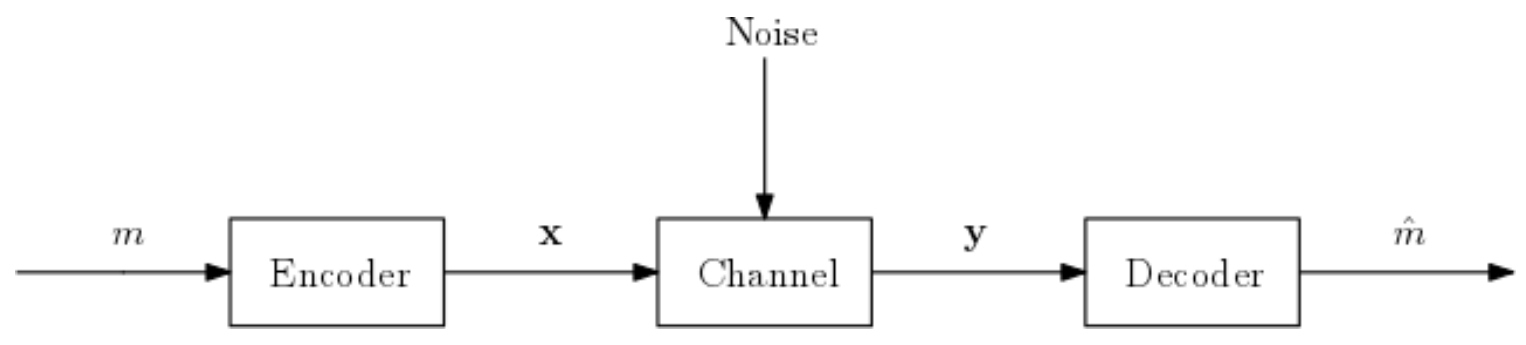

Figure 2.1: Essential communication system units. 
Definition 1. When there exists a permutation between matrix rows of any DMC $\operatorname{Pr}\{y \mid x\}$ we say that the DMC is symmetric.

For example, a DMC with transition matrix

$$
\operatorname{Pr}\{y \mid x\}=\left[\begin{array}{ll}
0.8 & 0.2 \\
0.2 & 0.8
\end{array}\right]
$$

is a symmetric DMC.

Definition 2. The DMC is known as binary-DMC (B-DMC) when its input alphabet has two elements.

Definition 3. The mutual information $\mathrm{I}(X ; Y)$ of a $D M C \operatorname{Pr}: \mathcal{X} \rightarrow \mathcal{Y}$ can be written as

$$
\mathrm{I}(X ; Y)=\sum_{y \in \mathcal{Y}} \sum_{x \in \mathcal{X}} p(x) \operatorname{Pr}\{y \mid x\} \log _{2} \frac{\operatorname{Pr}\{y \mid x\}}{\sum_{x^{\prime} \in \mathcal{X}} p\left(x^{\prime}\right) \operatorname{Pr}\left\{y \mid x^{\prime}\right\}}
$$

where $p(x)$ is the input distribution.

Definition 4. The information capacity of a $D M C \operatorname{Pr}: \mathcal{X} \rightarrow \mathcal{Y}$ can be written as

$$
\mathrm{C}=\max _{p(x)} \mathrm{I}(X ; Y)
$$

Definition 5. An $(M, N)$ code for the channel $\operatorname{Pr}: \mathcal{X} \rightarrow \mathcal{Y}$ has the following:

1. An index set $\{1,2, \ldots, M\}$, representing messages.

2. An encoding function $f:\{1,2, \ldots, M\} \rightarrow \mathcal{X}^{N}$, and the codeword is generated by setting $x=f(m), m \in\{1,2, \ldots, M\}$.

3. The rate $R$ of an $(M, N)$ code is defined as

$$
R=\frac{\log _{2} M}{N} \quad \text { bits } / \text { transmission }
$$

\subsection{Block Codes}

Encoding messages is the main principle of block coding so that the receiver is capable of detecting errors. Fig. 2.2 shows the block codes diagram. When the messages are 


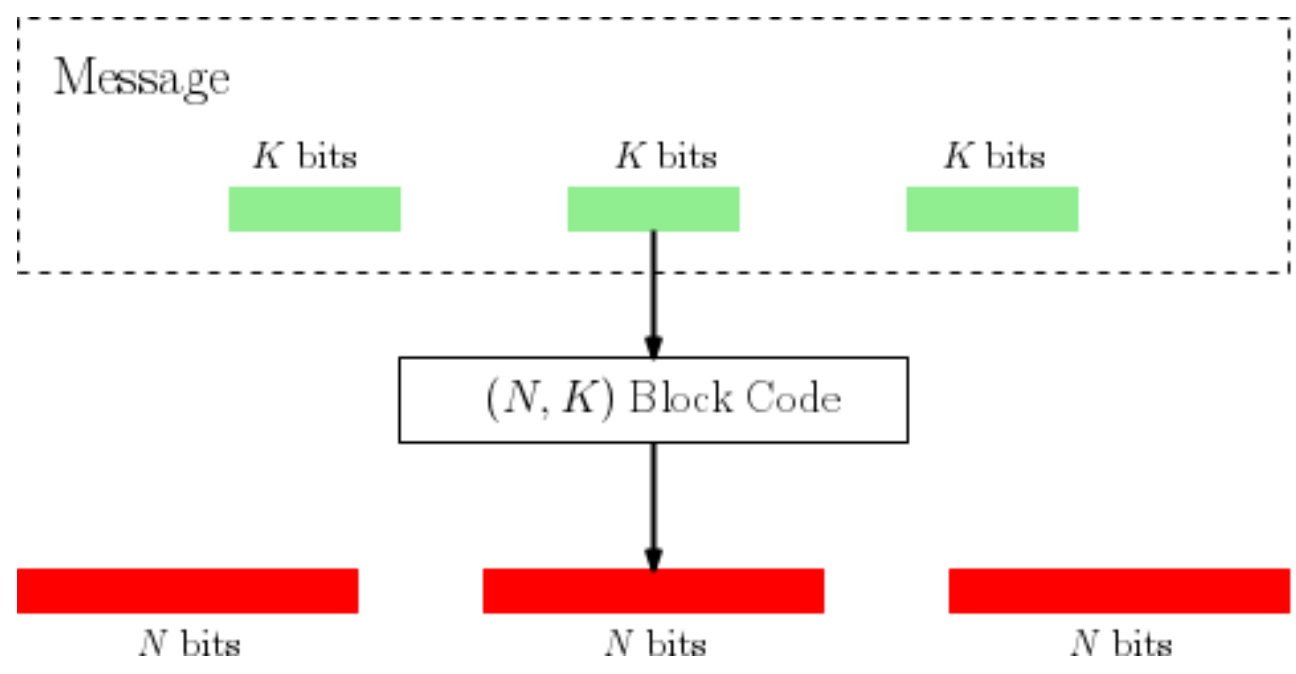

Figure 2.2: Block coding.

divided into $K$ bits and the code word length is $N$, then the set of $2^{K}$ codewords is represented as $(N, K)$ with $K / N=R$ is the code rate.

Single parity-check codes is one of the most important block codes that used to generate other most important codes like low-density parity-check codes (LDPC).

\subsubsection{Single Parity-Check Codes}

Assume a message word $\mathbf{m}=\left[\begin{array}{ll}m_{0} & m_{1}\end{array}\right]$ is encoded with a single parity check code, giving codeword $\mathbf{c}=\left[\begin{array}{lll}c_{0} & c_{1} & c_{2}\end{array}\right]$ according to $c_{0}=m_{0}, c_{1}=m_{1}$, and $c_{2}=m_{0} \oplus m_{1}$, where $\oplus$ denotes binary addition (exclusive OR). The codeword is transmitted over a binary-discrete memoryless channel (B-DMC) with transition probabilities $p\left(y_{i} \mid c_{i}\right)$ where $\boldsymbol{y}=\left[\begin{array}{lll}y_{0} & y_{1} & y_{2}\end{array}\right]$ is the received samples. The maximum a posterior (MAP) decision rule for $c_{i}$ is

$$
\hat{c}_{i}(\boldsymbol{y})=\underset{c_{i}}{\arg \max } p\left(c_{i} \mid \boldsymbol{y}\right),
$$

or

$$
p\left(c_{i}=0 \mid \boldsymbol{y}\right) \underset{0}{\stackrel{1}{\lessgtr}} p\left(c_{i}=1 \mid \boldsymbol{y}\right) .
$$

Equivalently the rule can be written as

$$
\frac{p\left(c_{i}=0 \mid \boldsymbol{y}\right)}{p\left(c_{i}=1 \mid \boldsymbol{y}\right)} \underset{0}{\stackrel{1}{\lessgtr}} 1,
$$


or

$$
\lambda_{i}=\ln \frac{p\left(c_{i}=0 \mid \boldsymbol{y}\right)}{p\left(c_{i}=1 \mid \boldsymbol{y}\right)} \underset{0}{\lessgtr} 0 .
$$

By using Bayes' rule we can express $\lambda_{i}$ as

$$
\lambda_{i}=\ln \frac{\frac{p\left(\boldsymbol{y} \mid c_{i}=0\right) p\left(c_{i}=0\right)}{p(\boldsymbol{y})}}{\frac{p\left(\boldsymbol{y} \mid c_{i}=1\right) p\left(c_{i}=1\right)}{p(\boldsymbol{y})}}=\ln \frac{p\left(\boldsymbol{y} \mid c_{i}=0\right)}{p\left(\boldsymbol{y} \mid c_{i}=1\right)}
$$

under the assumption that $p\left(c_{i}=0\right)=p\left(c_{i}=1\right)=\frac{1}{2}$.

Suppose we are interested in detecting $c_{2}$, then $\lambda_{2}$ can further be simplified as

$$
\begin{aligned}
\lambda_{2} & =\ln \frac{p\left(\boldsymbol{y} \mid c_{i}=0\right)}{p\left(\boldsymbol{y} \mid c_{i}=1\right)} \\
& =\ln \left(\frac{p\left(y_{0} \mid c_{0}=0\right) p\left(y_{1} \mid c_{1}=0\right)+p\left(y_{0} \mid c_{0}=1\right) p\left(y_{1} \mid c_{1}=1\right)}{p\left(y_{0} \mid c_{0}=0\right) p\left(y_{1} \mid c_{1}=1\right)+p\left(y_{0} \mid c_{0}=1\right) p\left(y_{1} \mid c_{1}=0\right)} \times \frac{p\left(y_{2} \mid c_{2}=0\right)}{p\left(y_{2} \mid c_{2}=1\right)}\right) \\
& =\ln \left(\frac{\frac{p\left(y_{0} \mid c_{0}=0\right)}{p\left(y_{0} \mid c_{0}=1\right)} \frac{p\left(y_{1} \mid c_{1}=0\right)}{p\left(y_{1} \mid c_{1}=1\right)}+1}{\frac{p\left(y_{0} \mid c_{0}=0\right)}{p\left(y_{0} \mid c_{0}=1\right)}+\frac{p\left(y_{1} \mid c_{1}=0\right)}{p\left(y_{1} \mid c_{1}=1\right)}}\right)+\ln \frac{p\left(y_{2} \mid c_{2}=0\right)}{p\left(y_{2} \mid c_{2}=1\right)} \\
& =\ln \frac{L_{0} L_{1}+1}{L_{0}+L_{1}}+\ln L_{2}
\end{aligned}
$$

where $L_{i}=\frac{p\left(y_{i} \mid c_{i}=0\right)}{p\left(y_{i} \mid c_{i}=1\right)}$

Let $\lambda_{i}=\ln L_{i}=\ln \frac{p\left(y_{i} \mid c_{i}=0\right)}{p\left(y_{i} \mid c_{i}=1\right)}$, then

$$
\begin{aligned}
\lambda_{2} & =\ln \frac{e^{\lambda_{0}^{(i)}} e^{\lambda_{1}^{(i)}}+1}{e^{\lambda_{0}^{(i)}}+e^{\lambda_{1}^{(i)}}}+\ln e^{\lambda_{2}^{(i)}} \\
& =\underbrace{\lambda_{2}^{(e)}}_{\text {Extrinsic LLR }}+\underbrace{\lambda_{2}^{(i)}}_{\text {Intrinsic LLR }},
\end{aligned}
$$

where

$$
\lambda_{2}^{(e)}=\ln \frac{e^{\lambda_{0}^{(i)}} e^{\lambda_{1}^{(i)}}+1}{e^{\lambda_{0}^{(i)}}+e^{\lambda_{1}^{(i)}}} .
$$

Extrinsic LLRs provide the reliability information generated from code constraints, whereas intrinsic LLRs provide the reliability information raised directly from channel observations [30]. To make a decision about bit $c_{2}$, the decoder adds these two LLRs to compute the total LLR, $\lambda_{2}$, and declares the bit zero if the sum is positive and one 
otherwise, as described by the rule (2.7).

We can use trigonometric identities to simplify (2.11). Since

$$
e^{\lambda_{0}^{(i)}} e^{\lambda_{1}^{(i)}}+1=\frac{1}{2}\left(e^{\lambda_{0}^{(i)}}+1\right)\left(e^{\lambda_{1}^{(i)}}+1\right)+\frac{1}{2}\left(e^{\lambda_{0}^{(i)}}-1\right)\left(e^{\lambda_{1}^{(i)}}-1\right)
$$

and

$$
e^{\lambda_{0}^{(i)}}+e^{\lambda_{1}^{(i)}}=\frac{1}{2}\left(e^{\lambda_{0}^{(i)}}+1\right)\left(e^{\lambda_{1}^{(i)}}+1\right)-\frac{1}{2}\left(e^{\lambda_{0}^{(i)}}-1\right)\left(e^{\lambda_{1}^{(i)}}-1\right)
$$

then

$$
\lambda_{2}^{(e)}=\ln \frac{1+\frac{\left(e^{\lambda_{0}^{(i)}}-1\right)\left(e^{\lambda_{1}^{(i)}}-1\right)}{\left(e^{\lambda_{0}^{(i)}}+1\right)\left(e^{\lambda_{1}^{(i)}}+1\right)}}{1-\frac{\left(e^{\lambda_{0}^{(i)}}-1\right)\left(e^{\lambda_{1}^{(i)}}-1\right)}{\left(e^{\lambda_{0}^{(i)}}+1\right)\left(e^{\lambda_{1}^{(i)}}+1\right)}} .
$$

Since

$$
\tanh \frac{x}{2}=\frac{e^{x}-1}{e^{x}+1}
$$

therefore

$$
\lambda_{2}^{(e)}=\ln \frac{1+\tanh \frac{\lambda_{0}^{(i)}}{2} \tanh \frac{\lambda_{1}^{(i)}}{2}}{1-\tanh \frac{\lambda_{0}^{(i)}}{2} \tanh \frac{\lambda_{1}^{(i)}}{2}}
$$

and also

$$
\tanh \frac{\lambda_{2}^{(e)}}{2}=\frac{e^{\lambda_{2}^{(e)}}-1}{e^{\lambda_{2}^{(e)}}+1} .
$$

By substituting (2.13) in (2.14) we get

$$
\begin{aligned}
\tanh \frac{\lambda_{2}^{(e)}}{2}= & \frac{\frac{1+\tanh \frac{\lambda_{0}^{(i)}}{2} \tanh \frac{\lambda_{1}^{(i)}}{2}}{1-\tanh \frac{\lambda_{0}^{(i)}}{2} \tanh \frac{\lambda_{1}^{(i)}}{2}}-1}{\frac{1+\tanh \frac{\lambda_{0}^{(i)}}{2} \tanh \frac{\lambda_{1}^{(i)}}{2}}{1-\tanh \frac{\lambda_{0}^{(i)}}{2} \tanh \frac{\lambda_{1}^{(i)}}{2}}+1} \\
= & \tanh \frac{\lambda_{0}^{(i)}}{2} \tanh \frac{\lambda_{1}^{(i)}}{2} .
\end{aligned}
$$

Hence

$$
\lambda_{2}^{(e)}=2 \tanh ^{-1}\left(\tanh \frac{\lambda_{0}^{(i)}}{2} \tanh \frac{\lambda_{1}^{(i)}}{2}\right) .
$$

A similar computation is followed to find the LLRs for $c_{0}$ and $c_{1}$ bits. So at all 


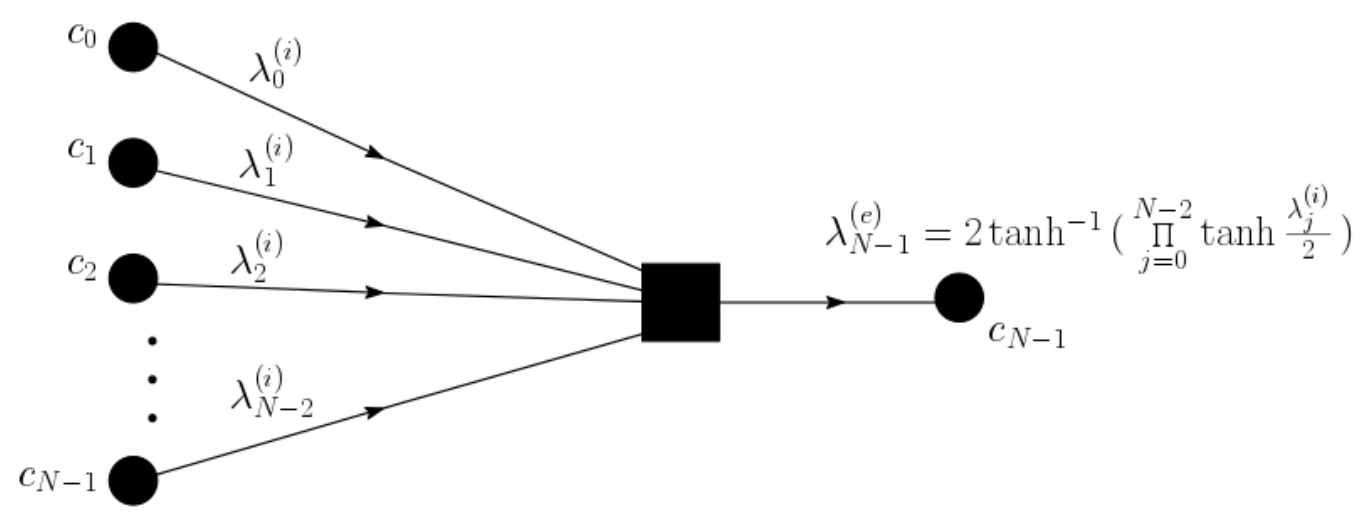

Figure 2.3: Message passing representation.

events, the extrinsic LLR of $k$ th bit is

$$
\lambda_{k}^{(e)}=2 \tanh ^{-1}\left(\prod_{j \neq k} \tanh \frac{\lambda_{j}^{(i)}}{2}\right)
$$

In Fig. 2.3, the rectangle shows the check node and the circle shows the variable node.

\subsection{Review of Polar Codes}

Over a B-DMC channel ${ }^{1}$, let $W: \mathcal{X} \rightarrow \mathcal{Y}$ with input alphabet $\mathcal{X}=\{0,1\}$ and $\mathcal{Y}$ is the output. Let $W(y \mid x)$ be the transition probability of $W$ for $x \in \mathcal{X}, y \in \mathcal{Y}$.

Definition 6. $W: \mathcal{X} \rightarrow \mathcal{Y}$ is symmetric over a $B-D M C$ if there exists a permutation $\pi: \mathcal{Y} \rightarrow \mathcal{Y}$ such that $\pi=\pi^{-1}$ and $W(y \mid+1)=W(\pi(y) \mid-1)$ for all $y \in \mathcal{Y}$.

Definition 7. The symmetric mutual information of a $B$-DMC $W: \mathcal{X} \rightarrow \mathcal{Y}$ is

\footnotetext{
${ }^{1}$ There are some binary memoryless symmetric channels of particular interest. Here we introduce these channels and give a brief illustration of them in Fig. 2.4.

- Binary erasure channel (BEC) with erasure probability $\epsilon$. The value of $\epsilon$ lies inside $[0,1]$.

- Binary symmetric channel (BSC) with cross over probability $\epsilon$. The value of $\epsilon$ lies inside $\left[0, \frac{1}{2}\right]$.

- Binary additive white Gaussian noise channel (BAWGNC) with noise variance $\sigma^{2}$. The value of $\sigma$ lies inside $(0, \infty)$.
} 
defined as

$$
\mathrm{I}(W)=\sum_{y \in \mathcal{Y}} \sum_{x \in \mathcal{X}} \frac{1}{2} W(y \mid x) \log _{2} \frac{W(y \mid x)}{\frac{1}{2} W(y \mid 0)+\frac{1}{2} W(y \mid 1)} .
$$

For a channel $W$ and under B-DMC, $N$ channels $\left(W_{N}^{(i)}\right)$ are reproduced via channel transformation [1] where polar codes are then able to get symmetric information rates. Channel transformation is done over the following steps.

\subsubsection{Channel Combining}

In this step, $\boldsymbol{W}_{N}: \mathcal{X}_{N} \rightarrow \mathcal{Y}_{N}$ is a vector channel which gathers all reproduced channels with $\boldsymbol{x}_{1}^{N}$ is an encoded channel input from the mapping $\boldsymbol{u}_{1}^{N} \rightarrow \boldsymbol{x}_{1}^{N}$ such that $\boldsymbol{x}_{1}^{N}=\boldsymbol{u}_{1}^{N} \mathbf{G}_{N}$, and $\boldsymbol{y}_{1}^{N}$ is the output vector. ${ }^{2}$

$$
\boldsymbol{W}_{N}\left(\boldsymbol{y}_{1}^{N} \mid \boldsymbol{u}_{1}^{N}\right)=\prod_{i=1}^{N} W\left(y_{i} \mid u_{i}\right)
$$

The generator matrix, $\mathbf{G}_{N}$, is used to generate the input vector. With $n=\log _{2} N$, suppose

$$
\mathbf{G}_{2}=\left[\begin{array}{ll}
1 & 0 \\
1 & 1
\end{array}\right] .
$$

\footnotetext{
${ }^{2}$ The notation $x_{a}^{b}$ refers to the vector $\left[\begin{array}{llll}x_{a} & x_{a-1} & \ldots & x_{b}\end{array}\right]$.
}

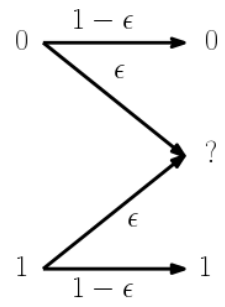

$\mathrm{BEC}$

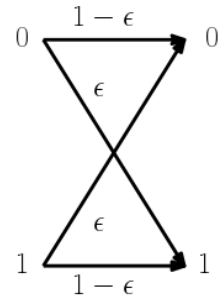

$\mathrm{BSC}$

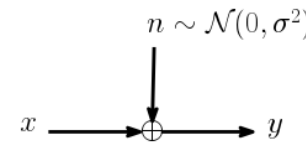

BAWGN

Figure 2.4: Left figure: the BEC with erasure probability $z$. An input symbol to this channel is erased with probability $z$ or passed through the channel with probability $1-z$. Middle figure: the BSC with cross-over probability $\epsilon$. An input to this channel is flipped with probability $\epsilon$ or passed through with probability $1-\epsilon$. Right figure: the BAWGN with noise variance $\sigma^{2}$. The output $y$ of this channel is the sum of its input $x \in\{0,1\}$ and a noise value $n$ which is a Gaussian random variable with mean 0 and variance $\sigma^{2}$. 
Then

$$
\begin{aligned}
\mathbf{G}_{N} & =\mathbf{G}_{\frac{N}{2}}^{\otimes 2} \\
& =\left(\mathbf{G}_{\frac{N}{4}}^{\otimes 2}\right)^{\otimes 2} \\
& \vdots \\
& =\mathbf{G}_{2}^{\otimes n}
\end{aligned}
$$

where $\otimes$ denotes the Kronecker product.

As in Fig. 2.5 a, we have

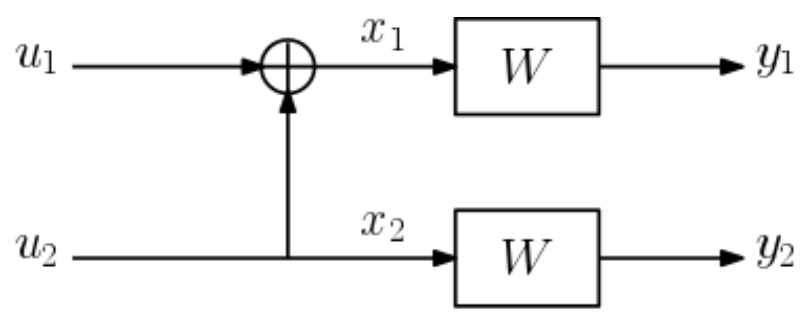

a

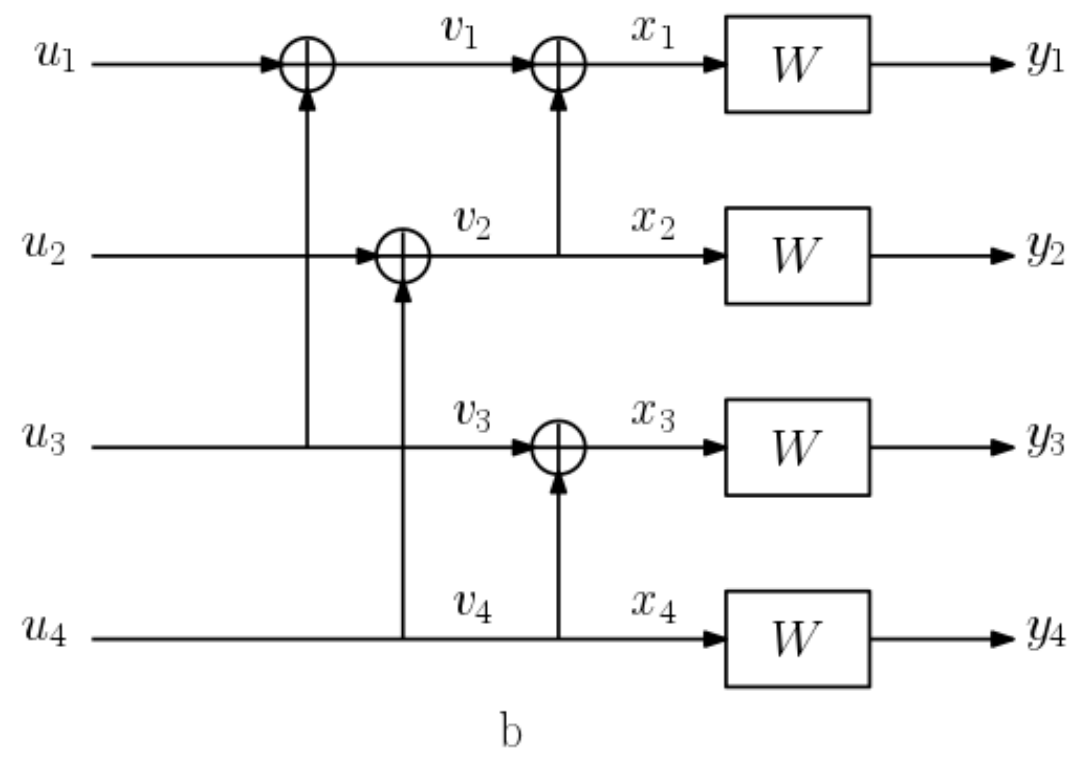

Figure 2.5: Channels a) $\boldsymbol{W}_{2}$, b) $\boldsymbol{W}_{4}$. 


$$
\begin{aligned}
\boldsymbol{x}_{1}^{2} & =\boldsymbol{u}_{1}^{2} \mathbf{G}_{2} \\
& =\left(u_{1} \oplus u_{2}, u_{2}\right) .
\end{aligned}
$$

Similarly in Fig. 2.5 b, the mapping from $\boldsymbol{u}_{1}^{4} \rightarrow \boldsymbol{x}_{1}^{4}$ can be formulated as

$$
\begin{aligned}
\boldsymbol{x}_{1}^{4} & =\boldsymbol{u}_{1}^{4} \mathbf{G}_{4} \\
& =\boldsymbol{u}_{1}^{4} \mathbf{G}_{2}^{\otimes 2} \\
& =\left(\boldsymbol{u}_{1}^{2}, \boldsymbol{u}_{3}^{4}\right)\left[\begin{array}{ll}
\mathbf{G}_{2} & 0 \\
\mathbf{G}_{2} & \mathbf{G}_{2}
\end{array}\right] \\
& =\left(\left(\boldsymbol{u}_{1}^{2} \oplus \boldsymbol{u}_{3}^{4}\right) \mathbf{G}_{2}, \boldsymbol{u}_{3}^{4} \mathbf{G}_{2}\right) .
\end{aligned}
$$

And in general (see Fig. 2.6)

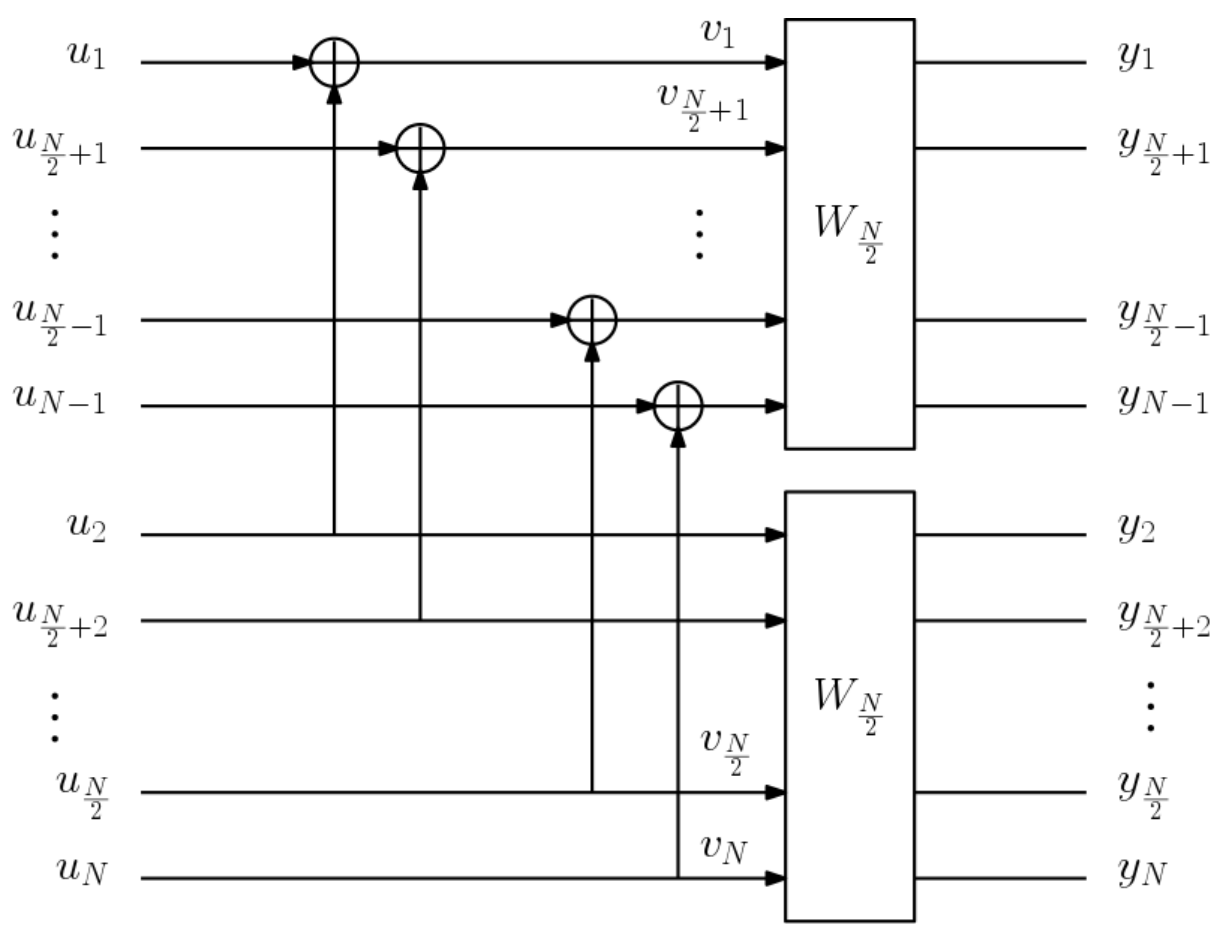

Figure 2.6: Channel $\boldsymbol{W}_{N}$. 


$$
\begin{aligned}
\boldsymbol{x}_{1}^{N} & =\boldsymbol{u}_{1}^{N} \mathbf{G}_{N} \\
& =\boldsymbol{u}_{1}^{N} \mathbf{G}_{\frac{N}{2}}^{\otimes 2} \\
& =\left(\left(\boldsymbol{u}_{1}^{\frac{N}{2}} \oplus \boldsymbol{u}_{\frac{N}{2}}^{N}\right) \mathbf{G}_{\frac{N}{2}}, \boldsymbol{u}_{\frac{N}{2}}^{N} \mathbf{G}_{\frac{N}{2}}\right) .
\end{aligned}
$$

\subsubsection{Channel Splitting}

In this step the vector $\boldsymbol{W}_{N}$ splits into $N$ channels $\boldsymbol{W}_{N}^{(i)}[1]$ [33]

$$
W_{N}^{(i)}\left(\boldsymbol{y}_{1}^{N}, \boldsymbol{u}_{1}^{N} \mid u_{i}\right)=\sum_{\boldsymbol{u}_{i+1}^{N} \in \mathcal{X}^{N-i}} \frac{1}{2^{N-1}} \boldsymbol{W}_{N}\left(\boldsymbol{y}_{1}^{N} \mid \boldsymbol{u}_{1}^{N}\right)
$$

\subsubsection{Recursive Channel Transformation}

For the channel $W$, the two channels $W_{2}^{(1)}$ and $W_{2}^{(2)}$ can be calculated recursively in the following way.

$$
W_{2}^{(1)}\left(\boldsymbol{y}_{1}^{2} \mid u_{1}\right)=\sum_{u_{2}} \frac{1}{2} W\left(y_{1} \mid u_{1} \oplus u_{2}\right) W\left(y_{2} \mid u_{2}\right)
$$

and

$$
W_{2}^{(2)}\left(\boldsymbol{y}_{1}^{2}, u_{1} \mid u_{2}\right)=\frac{1}{2} W\left(y_{1} \mid u_{1} \oplus u_{2}\right) W\left(y_{2} \mid u_{2}\right) .
$$

At the $i$ th step, the last two equations can be re-expressed as

$$
\begin{aligned}
& W_{2^{i}}^{2 j-1}\left(\boldsymbol{y}_{1}^{2^{i}}, u_{1}^{2 j-2} \mid u_{2 j-1}\right)= \\
& \sum_{u_{2 j}} \frac{1}{2} W_{2^{i-1}}^{j}\left(\boldsymbol{y}_{1}^{2^{i-1}}, \boldsymbol{u}_{1, o}^{2 j-2} \oplus \boldsymbol{u}_{1, e}^{2 j-2} \mid u_{2 j-1} \oplus u_{2 j}\right) W_{2^{i-1}}^{j}\left(\boldsymbol{y}_{2^{i-1}+1}^{2^{i}}, \boldsymbol{u}_{1, e}^{2 j-2} \mid u_{2 j}\right) \\
& W_{2^{i}}^{2 j}\left(\boldsymbol{y}_{1}^{2^{i}}, u_{1}^{2 j-1} \mid u_{2 j}\right)= \\
& \frac{1}{2} W_{2^{i-1}}^{j}\left(\boldsymbol{y}_{1}^{2^{i-1}}, \boldsymbol{u}_{1, o}^{2 j-2} \oplus \boldsymbol{u}_{1, e}^{2 j-2} \mid u_{2 j-1} \oplus u_{2 j}\right) W_{2^{i-1}}^{j}\left(\boldsymbol{y}_{2^{i-1}+1}^{2^{i}}, \boldsymbol{u}_{1, e}^{2 j-2} \mid u_{2 j}\right)
\end{aligned}
$$

where $j=1, \ldots, 2^{i-1}, \boldsymbol{u}_{1, o}^{2 j-2}=\left(u_{1}, u_{3}, \ldots, u_{2 j-1}\right)$, and $\boldsymbol{u}_{1, e}^{2 j-2}=\left(u_{2}, u_{4}, \ldots, u_{2 j-2}\right)$.

Lemma 1. For $W$ is a $B-D M C$, we have

$$
\begin{aligned}
& \mathrm{I}\left(W_{2^{i}}^{2 j-1}\right) \leqslant \mathrm{I}\left(W_{2^{i-1}}^{j}\right) \leqslant \mathrm{I}\left(W_{2^{i}}^{2 j}\right), \\
& \mathrm{I}\left(W_{2^{i}}^{2 j-1}\right)+\mathrm{I}\left(W_{2^{i}}^{2 j}\right)=2 \mathrm{I}\left(W_{2^{i-1}}^{j}\right)
\end{aligned}
$$


Proof. For the proof, assume $N=2$. If the answer satisfies then we can generalize the case.

Referring to Fig. 2.5 a the second equality follows from the chain rule of mutual information as

$$
\mathrm{I}\left(W_{2}^{1}\right)+\mathrm{I}\left(W_{2}^{2}\right)=\mathrm{I}\left(\mathrm{U}_{1} ; \mathrm{Y}_{1}\right)=\mathrm{I}\left(\mathrm{X}_{1} ; \mathrm{Y}_{1}\right)=2 \mathrm{I}(W) .
$$

For the first inequality, combine the second equality with

$$
\begin{aligned}
\mathrm{I}\left(W_{2}^{2}\right) & =\mathrm{I}\left(\mathrm{U}_{2} ; \mathrm{Y}_{1}, \mathrm{U}_{1}\right) \\
& =\mathrm{H}\left(\mathrm{U}_{2}\right)-\mathrm{H}\left(\mathrm{U}_{2} \mid \mathrm{Y}_{1}, \mathrm{U}_{1}\right) \\
& \geqslant \mathrm{H}\left(\mathrm{U}_{2}\right)-\mathrm{H}\left(\mathrm{U}_{2} \mid \mathrm{Y}_{2}\right) \\
& =\mathrm{I}(W) .
\end{aligned}
$$

\subsection{Phenomenon of Channel Polarization}

Using the chain rule of mutual information, we can write

$$
\begin{aligned}
\mathrm{I}\left(\mathbf{X}_{1}^{N}, \mathbf{U}_{1}^{N} ; \mathbf{Y}_{1}^{N}\right) & =\mathrm{I}\left(\mathbf{X}_{1}^{N} ; \mathbf{Y}_{1}^{N}\right)+\mathrm{I}\left(\mathbf{U}_{1}^{N} \mid \mathbf{X}_{1}^{N} ; \mathbf{Y}_{1}^{N}\right) \\
& =\mathrm{I}\left(\mathbf{U}_{1}^{N} ; \mathbf{Y}_{1}^{N}\right)+\mathrm{I}\left(\mathbf{X}_{1}^{N} \mid \mathbf{U}_{1}^{N} ; \mathbf{Y}_{1}^{N}\right) .
\end{aligned}
$$

because of

$$
\mathrm{I}\left(\mathbf{U}_{1}^{N} \mid \mathbf{X}_{1}^{N} ; \mathbf{Y}_{1}^{N}\right)=\mathrm{I}\left(\mathbf{X}_{1}^{N} \mid \mathbf{U}_{1}^{N} ; \mathbf{Y}_{1}^{N}\right)=0
$$

then

$$
\mathrm{I}\left(\mathbf{X}_{1}^{N} ; \mathbf{Y}_{1}^{N}\right)=\mathrm{I}\left(\mathbf{U}_{1}^{N} ; \mathbf{Y}_{1}^{N}\right)
$$

Therefore,

$$
\begin{aligned}
\mathrm{I}\left(\boldsymbol{W}_{N}\right) & =\mathrm{I}\left(\mathbf{U}_{1}^{N} ; \mathbf{Y}_{1}^{N}\right) \\
& =\mathrm{I}\left(\mathbf{X}_{1}^{N} ; \mathbf{Y}_{1}^{N}\right) \\
& =N \mathrm{I}(W) .
\end{aligned}
$$

As an example, the input $x$ in Fig. 2.7 a takes value 0 or 1 . If the transition probability is 0.5 , then the achieved rate is $\mathrm{I}(W)=1-0.5=0.5$. Similarly in Fig. $2.7 \mathrm{~b}$, there are two inputs $x_{1}, x_{2}$ and two outputs $y_{1}, y_{2}$ where the achieved rate is 


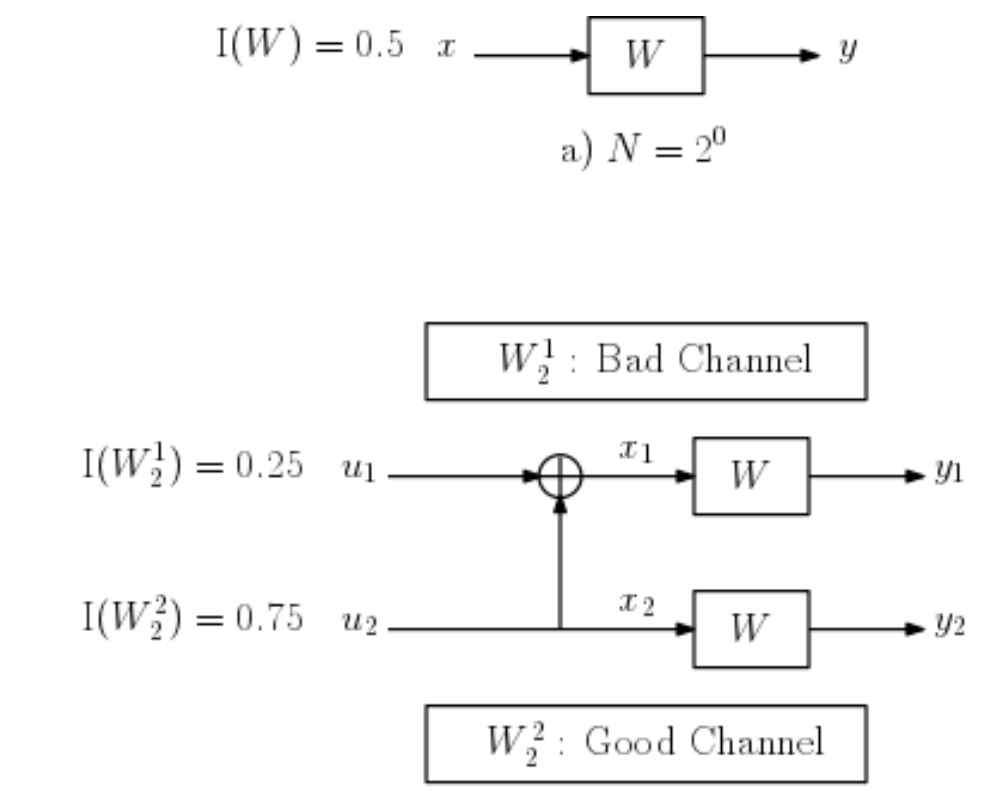

b) A Butterfly. $N=2^{1}$

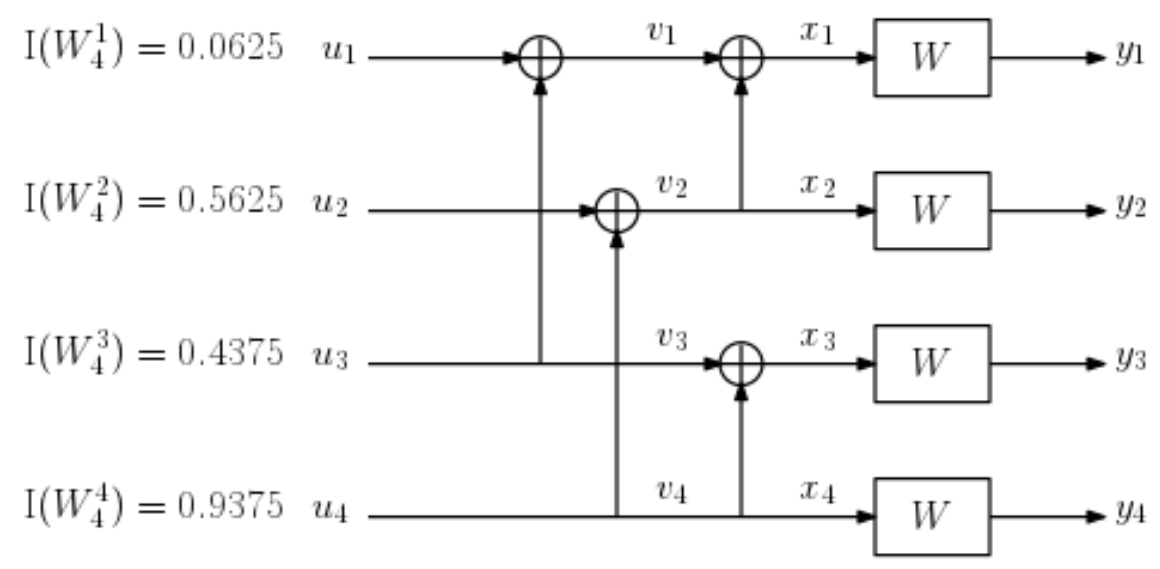

c) $N=2^{2}$

Figure 2.7: Channel polarization examples probability $\frac{1}{2}$ a) $N=2^{0}$; b) $N=2^{1}$, and c) $N=2^{2}$.

$2 \mathrm{I}(W)$. By using the chain rule, two channels are created $W_{2}^{1}$ and $W_{2}^{2}$. After channel combining and channel splitting, two independent channels with the same reliability are transformed into two polarized channels with new achieved rates (Fig. 2.7 b) $\mathrm{I}\left(W_{2}^{1}\right)+\mathrm{I}\left(W_{2}^{2}\right)=2 \mathrm{I}(W)$. Now, since $\mathrm{I}\left(W_{2}^{2}\right)=2 \mathrm{I}(W)-\mathrm{I}^{2}(W)$ where $\mathrm{I}\left(W_{2}^{1}\right)=\mathrm{I}^{2}(W)$ therefore $\mathrm{I}\left(W_{2}^{1}\right)$ has a smaller capacity than the given $W$, whereas the good channel 
$\mathrm{I}\left(W_{2}^{2}\right)$ has a larger capacity, that is $\mathrm{I}\left(W_{2}^{1}\right) \leqslant \mathrm{I}(W) \leqslant \mathrm{I}\left(W_{2}^{2}\right)$. Specifically, with $\mathrm{I}(W)$ the achieving rates of two polarized channels are $\mathrm{I}\left(W_{2}^{1}\right)=0.25$ and $\mathrm{I}\left(W_{2}^{2}\right)=0.75$. Moreover, new four channels $W_{4}^{1}, W_{4}^{2}, W_{4}^{3}$, and $W_{4}^{4}$ are derived from $W_{2}^{1}$ and $W_{2}^{2}$ as shown in Fig. 2.7 c. So, the $W_{2}^{1}$ channel is used instead of channel $W$ and the capacities of of channels $W_{4}^{1}$ and $W_{4}^{2}$ are obtained, that is $\mathrm{I}\left(W_{4}^{1}\right)=\mathrm{I}^{2}\left(W_{2}^{1}\right)=0.0625$ and $\mathrm{I}\left(W_{4}^{2}\right)=2 \mathrm{I}\left(W_{2}^{1}\right)-\mathrm{I}^{2}\left(W_{2}^{1}\right)=0.4375$. Similarly, the achieving rates of channels $W_{4}^{3}$, and $W_{4}^{4}$ are $\mathrm{I}\left(W_{4}^{3}\right)=\mathrm{I}^{2}\left(W_{2}^{2}\right)=0.5625$ and $\mathrm{I}\left(W_{4}^{4}\right)=2 \mathrm{I}\left(W_{2}^{2}\right)-\mathrm{I}^{2}\left(W_{2}^{2}\right)=0.9375$.

\subsection{Polar Codes Encoding}

For a B-DMC $W$, let $\mathcal{F} \in[N]$ indicates the channels indices whose mutual information is almost 0 [32]. Like channels are unable to transmit information bits dependably, and will freeze their corresponding input $u_{\mathcal{F}}$ to specific values (zeros for example) that are assumed to be known at both the encoder and decoder. This set is known as the frozen set. The complement of $u_{\mathcal{F}}, u_{\mathcal{F}^{c}}$, instead is used to transmit data and thus $u_{\mathcal{F}^{c}}$ is the information (data) bits. Fig. 2.8 is an example which shows the encoding process for an $(N, K)=(8,4)$ polar code designed with a transition probability of $\frac{1}{2}$.

Because of the recursive structure of $\mathbf{G}_{N}$, the computational complexity of polar codes encoding algorithm is found to be $O\left(N^{2}\right)$, which is the complexity of vectormatrix operations, to $O\left(N \log _{2} N\right)$. The encoding here is done stage by stage for $n=\log _{2} N$ stages with complexity of $O(N)$ per stage [34].

\subsection{Successive Cancellation Decoding of Polar Codes}

In his work, Arikan presented that SC decoding can be realized via code factor graph with a structure has almost the form that of the Fast Fourier Transform. In SC algorithm, each bit depends on some antecedent bits through the trellis of polar codes.

Let $\boldsymbol{u}_{1}^{N}$ is an input sequence, $\boldsymbol{x}_{1}^{N}$ is the corresponding encoded sequence and $\boldsymbol{y}_{1}^{N}$ is the output one. $u_{j}$ is estimated according to the values of $\boldsymbol{y}$ and $\hat{\boldsymbol{u}}_{1}^{j-1}$ (see Fig. 2.9). Suppose the likelihood ratio $(L)$ is: 


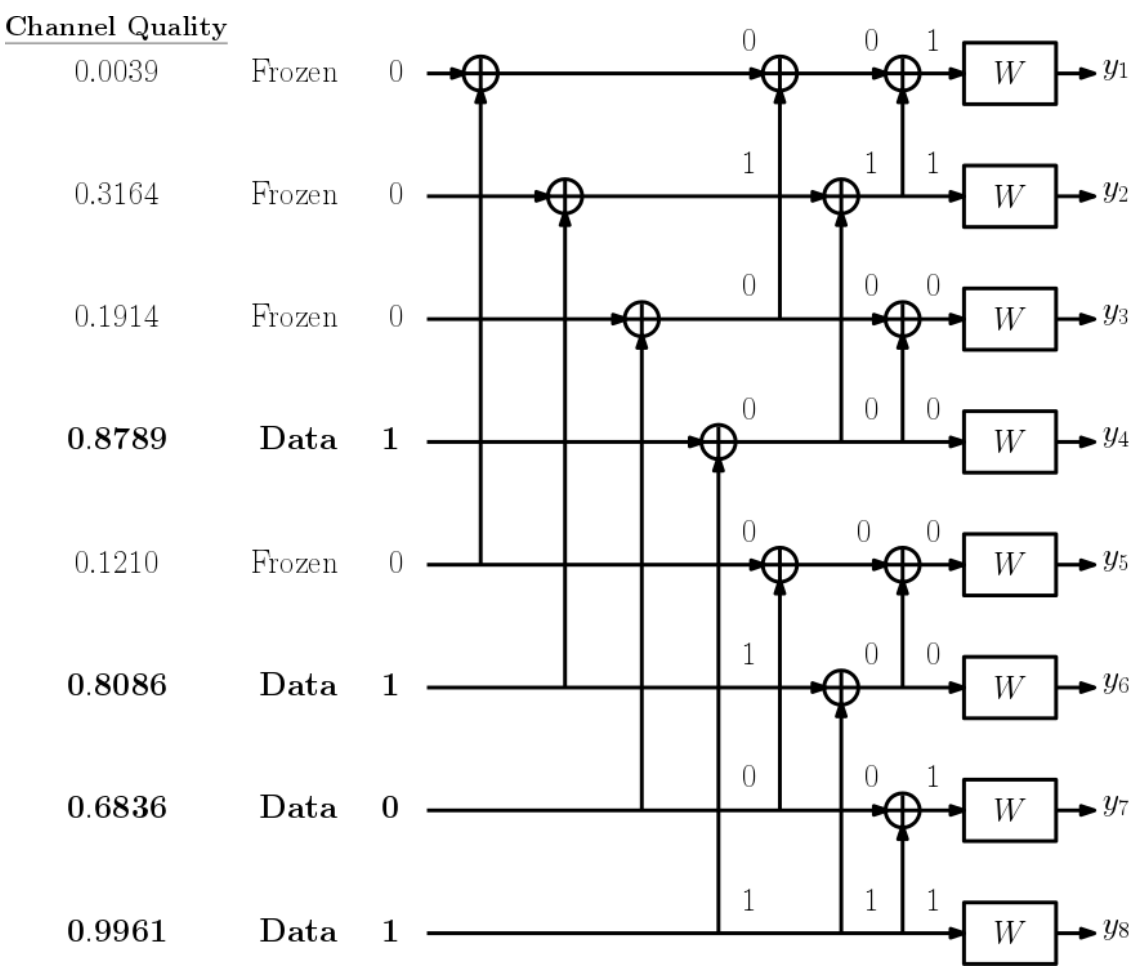

Figure 2.8: Encoding algorithm for an $(8,4)$ polar code.

$$
L_{N}^{2 j-1}\left(\boldsymbol{y}_{1}^{N}, \hat{\boldsymbol{u}}_{1}^{2 j-2}\right)=\frac{W_{N}^{2 j-1}\left(\boldsymbol{y}_{1}^{N}, \hat{\boldsymbol{u}}_{1}^{2 j-2} \mid u_{j}=0\right)}{W_{N}^{2 j-1}\left(\boldsymbol{y}_{1}^{N}, \hat{\boldsymbol{u}}_{1}^{2 j-2} \mid u_{j}=1\right)}
$$

$\boldsymbol{u}_{j}$ is then estimated as:

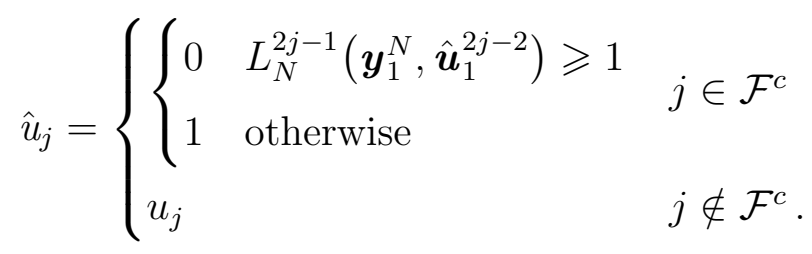

Now, let $s_{1, j}=\hat{u}_{j}$ and the $\log$-likelihood ratio $(\lambda)$ is $\lambda_{1, j}=\lambda\left(\hat{u}_{j}\right)$, then according to $(2.13-2.16)$, and as in Fig. 2.9 the updated soft message and decision condition can be expressed as follows [36]:

$$
\begin{aligned}
\lambda_{i, j} & =\lambda_{i+1, j} \boxplus \lambda_{i+1, j+2^{i-1}} \\
& =2 \tanh ^{-1}\left(\tanh \frac{\lambda_{i+1, j}}{2} \tanh \frac{\lambda_{i+1, j+2^{i-1}}}{2}\right)
\end{aligned}
$$




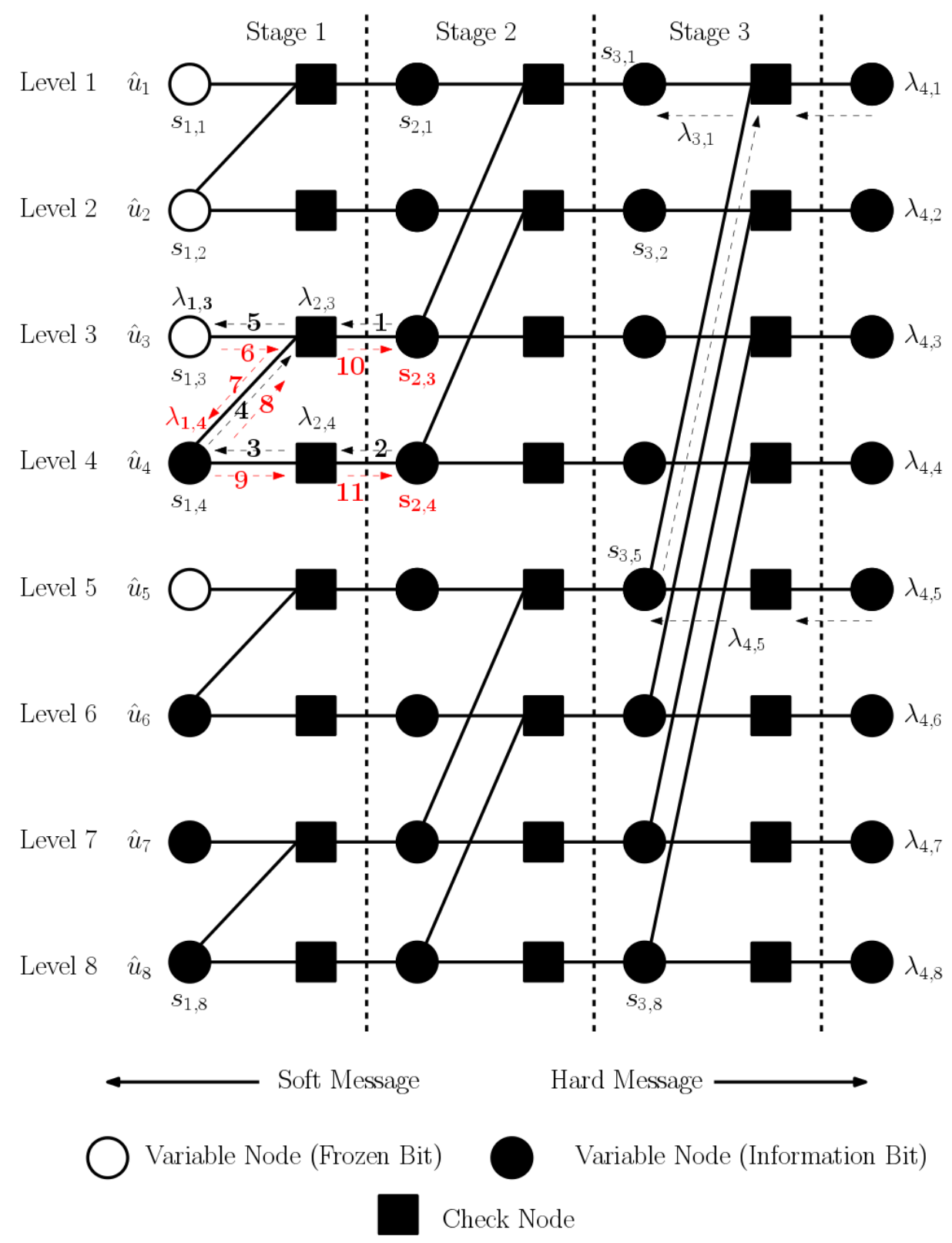

Figure 2.9: Trellis representations of polar codes.

in case of

$$
\left\lfloor\frac{j-1}{2^{i-1}}\right\rfloor \bmod 2=0,
$$

where $\boxplus$ is the boxplus operator with $\lambda \boxplus \infty=\lambda, \lambda \boxplus-\infty=-\lambda$, and $\lambda \boxplus 0=0$. Otherwise:

$$
\lambda_{i, j}=\left(1-2 s_{i, j-2^{i-1}}\right) \lambda_{i+1, j-2^{i-1}}+\lambda_{i+1, j}
$$


where the hard message $s_{1, j}$ is updated as:

$$
s_{i+1, j}= \begin{cases}s_{i, j} \oplus s_{i, j+2^{i-1}} & \text { if }\left\lfloor\frac{j-1}{2^{i-1}}\right\rfloor \bmod 2=0 \\ s_{i, j} & \text { otherwise }\end{cases}
$$

In the trellis structure, the SC decoder performs the soft messages calculations which pass through the trellis from right to left; while the hard messages are calculated and propagated in the reverse direction. As an instance, the soft message $\lambda_{3,1}$ in Fig. 2.9 is computed by using $\lambda_{4,1}$ and $\lambda_{4,5}$ according to (2.36) and then to (2.35) as follows: To calculate $\lambda_{3,1}$, we have $i=3$ and $j=1$. Then because of $\left\lfloor\frac{1-1}{2^{3-1}}\right\rfloor \bmod 2=$ $\left\lfloor\frac{0}{4}\right\rfloor \bmod 2=0$ we get $\lambda_{3,1}=2 \tanh ^{-1}\left(\tanh \frac{\lambda_{4,1}}{2} \tanh \frac{\lambda_{4,5}}{2}\right)$.

Moreover, soft messages $\lambda_{2,3}$ and $\lambda_{2,4}$ are sent to the related check nodes after step 1 and 2 (illustrated by the arrows and numbers), respectively. Then after steps $3 \sim 5$, the soft message $\lambda_{1,3}$ is obtained as $\lambda_{1,3}=2 \tanh ^{-1}\left(\tanh \frac{\lambda_{2,3}}{2} \tanh \frac{\lambda_{2,4}}{2}\right)$ where $\left\lfloor\frac{3-1}{2^{1-1}}\right\rfloor \bmod 2=0$. However, no matter what value $\lambda_{1,3}$ takes, the corresponding hard message $s_{1,3}=0$ is sent back to the check node because bit $u_{3}$ is a frozen bit. Then soft message $\lambda_{1,4}$ is calculated in step 6 (red color) by using (2.37) with the hard message $s_{1,3}=0$ and soft messages $\lambda_{2,4}$ and $\lambda_{2,3}$ as: $\left\lfloor\frac{4-1}{2^{1-1}}\right\rfloor \bmod 2=1$ gives $\lambda_{1,4}=\left(1-2 s_{1,3}\right) \lambda_{2,3}+\lambda_{2,4}=\lambda_{2,3}+\lambda_{2,4}$. The corresponding hard message $s_{1,4}$ is sent back to the check nodes in steps 8 and 9, respectively. In steps 10 and 11, the hard message $s_{2,3}$ and $s_{2,4}$ (in red) have been determined according to (2.38) as: $s_{2,3}=s_{1,3} \oplus s_{1,4}=0 \oplus s_{1,4}=s_{1,4}$ and $s_{2,4}=s_{1,4}$.

Fig. (2.10) and Fig. (2.11) show the transmission process on a BEC $(\epsilon=0.2)$ at $R=0.5$ and with $N=8$. Each component of the received sequence is erased (has unknown value) with probability less than $\epsilon$.

Those update conditions are complex to realize in hardware because they contain complex operations like exponentials; therefore in Chapter 3 we are going to simplify the function expressed in (2.37) more than what is presented in the literature.

Let us now look at the SC decoding complexity [1] [35]. Using (2.28) and (2.29) 


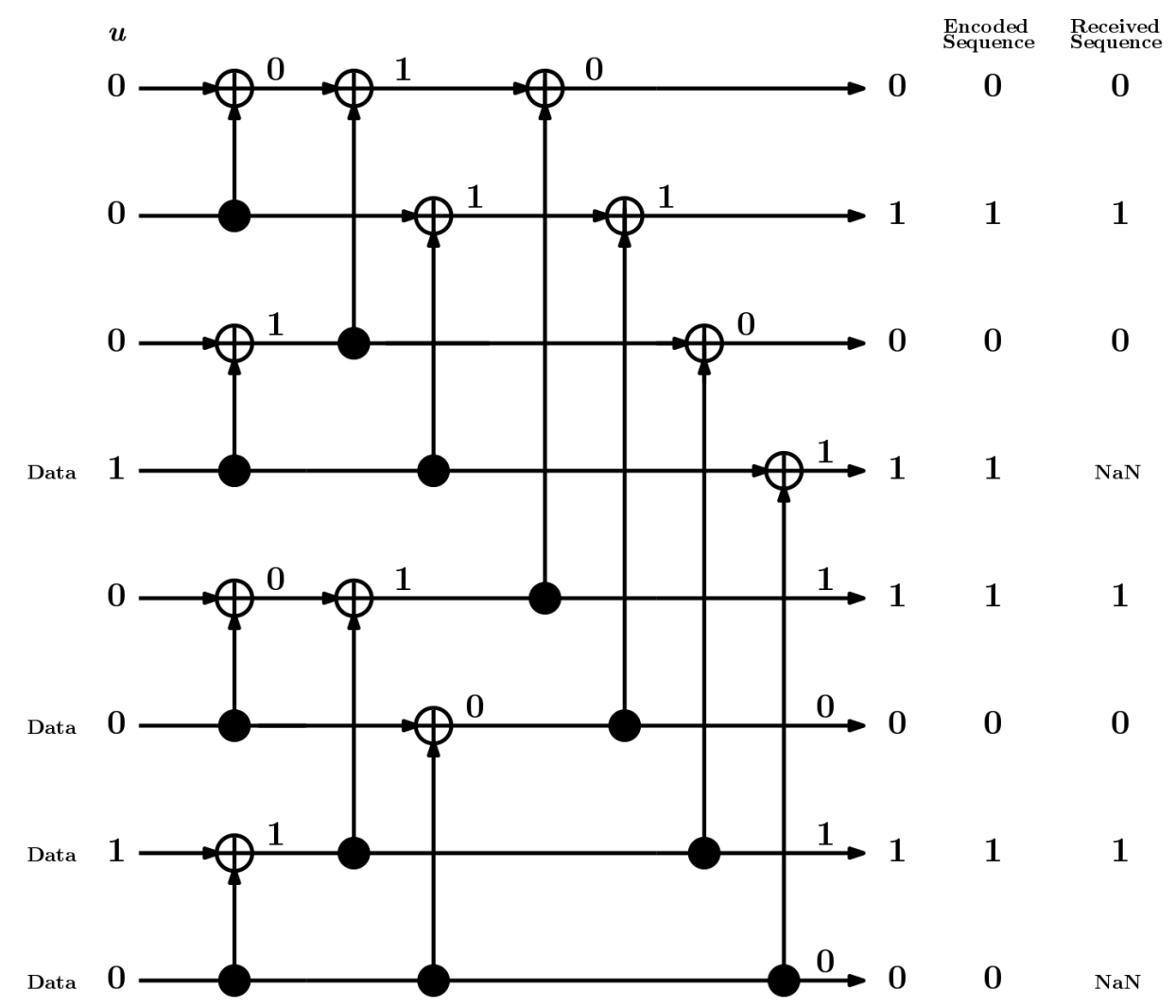

Figure 2.10: Polar encoder with $N=8$ and $R=0.5$.

we can prove that

$$
\begin{aligned}
L_{N}^{2 j-1}\left(\boldsymbol{y}_{1}^{N}, \hat{\boldsymbol{u}}_{1}^{2 j-2}\right) & =\frac{1+L_{\frac{N}{2}}^{j}\left(\boldsymbol{y}_{1}^{\frac{N}{2}}, \hat{\boldsymbol{u}}_{1, o}^{2 j-2} \oplus \hat{\boldsymbol{u}}_{1, e}^{2 j-2}\right) L_{\frac{N}{2}}^{j}\left(\boldsymbol{y}_{\frac{N}{2}+1}^{N}, \hat{\boldsymbol{u}}_{1, e}^{2 j-2}\right)}{L_{\frac{N}{2}}^{j}\left(\boldsymbol{y}_{1}^{\frac{N}{2}}, \hat{\boldsymbol{u}}_{1, o}^{2 j-2} \oplus \hat{\boldsymbol{u}}_{1, e}^{2 j-2}\right)+L_{\frac{N}{2}}^{j}\left(\boldsymbol{y}_{\frac{N}{2}+1}^{N}, \hat{\boldsymbol{u}}_{1, e}^{2 j-2}\right)}, \\
L_{N}^{2 j}\left(\boldsymbol{y}_{1}^{N}, \hat{\boldsymbol{u}}_{1}^{2 j-1}\right) & =L_{\frac{N}{2}}^{j}\left(\boldsymbol{y}_{1}^{\frac{N}{2}}, \hat{\boldsymbol{u}}_{1, o}^{2 j-2} \oplus \hat{\boldsymbol{u}}_{1, e}^{2 j-2}\right)^{1-2 \hat{\boldsymbol{u}}_{2 j-1}} L_{\frac{N}{2}}^{j}\left(\boldsymbol{y}_{\frac{N}{2}+1}^{N}, \hat{\boldsymbol{u}}_{1, e}^{2 j-2}\right) .
\end{aligned}
$$

Therefore, $L_{N}^{j}$ can be implemented with $O(N)$ computations as

$$
\begin{gathered}
L_{\frac{N}{2}}^{j}\left(\boldsymbol{y}_{1}^{\frac{N}{2}}, \hat{\boldsymbol{u}}_{1, o}^{2 j-2} \oplus \hat{\boldsymbol{u}}_{1, e}{ }^{2 j-2}\right): i \in\left[0,1, \ldots, \frac{N}{2}\right], \\
L_{\frac{N}{2}}^{j}\left(\boldsymbol{y}_{\frac{N}{2}+1}^{N}, \hat{\boldsymbol{u}}_{1, e}^{2 j-2}\right): i \in\left[0,1, \ldots, \frac{N}{2}\right] .
\end{gathered}
$$

The trellis structure contains $\frac{N}{2} \log _{2} N$ units and requires $O\left(N \log _{2} N\right)$ memory units to do the overall SC decoding algorithm.

Finally, several alternative decoding schemes have been proposed to improve the 


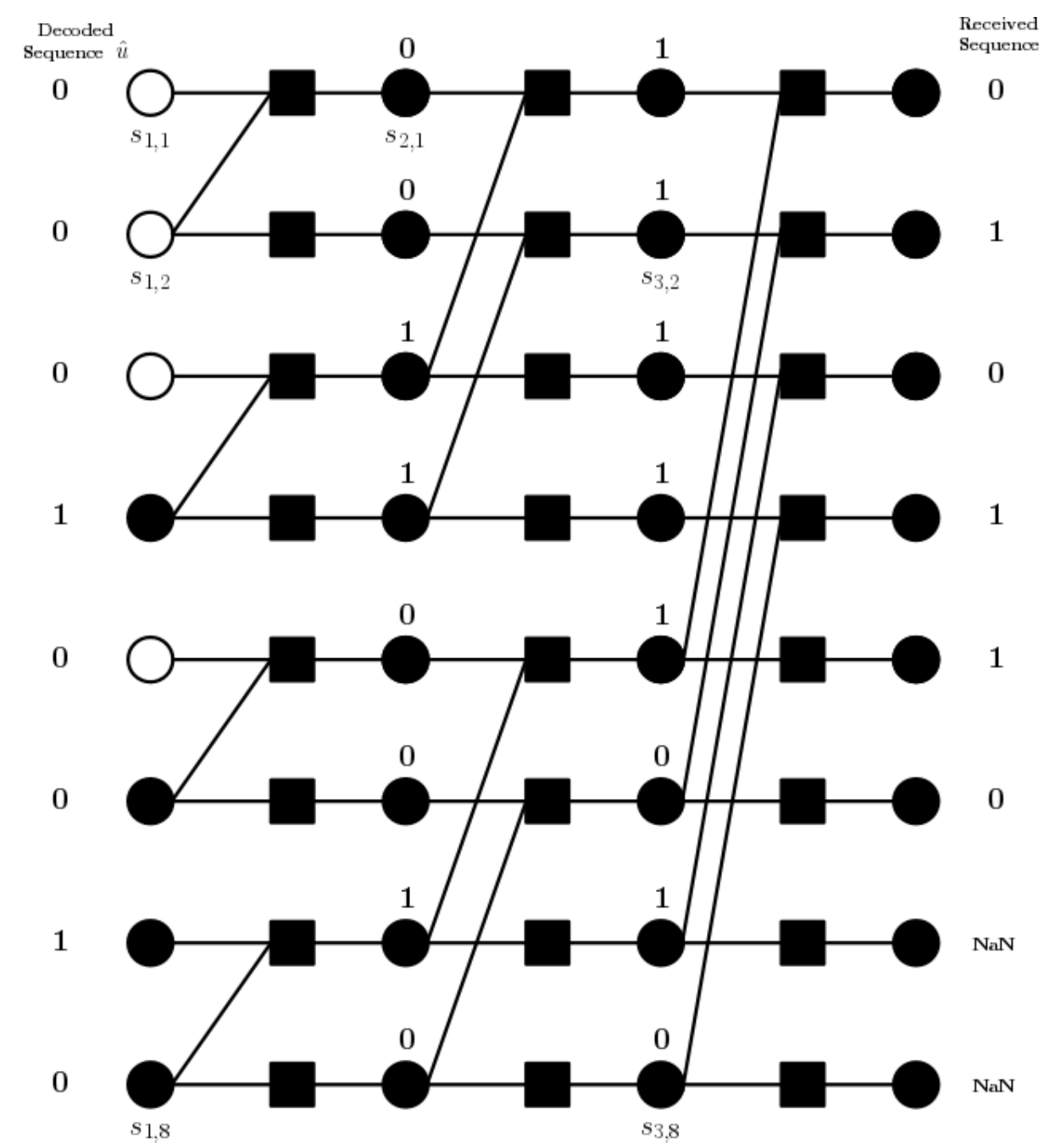

Figure 2.11: SC polar decoder with $N=8$ and $R=0.5$.

performance of polar codes, such as successive cancellation list/stack (SCL/SCS) and belief propagation (BP) decoding algorithms [36]. We will mention to these schemes in the introduction of the next chapter.

\subsection{Summary}

In this chapter, we gave a brief review of standard polar codes for B-DMCs, which include channel polarization, the encoding scheme, and the construction method. The $\mathrm{SC}$ decoding technique is investigated from the theoretical and practical viewpoints. 


\section{Chapter 3}

\section{Corrected NMS SC Decoding of Polar Codes}

Although polar codes have outstanding asymptotic behaviour, the error rate performance is not as competitive as LDPC or turbo codes under SC decoding. For this, some modified versions of the SC decoding algorithm were introduced either to improve the performance or to reduce the complexity and provide an efficient hardware implementation.

In [37], it shows that the belief propagation (BP) decoder can perform better than the $\mathrm{SC}$ decoder but there is still a performance gap with respect to that of LDPC decoders. A successive cancellation list (SCL) decoder is proposed after this as an improved version of SC decoding which performs almost the same as the optimal ML decoding at high SNR, with acceptable complexity [4]. With the help of a cyclic redundancy check (CRC) code, SCL decoders even outperforme that of turbo codes. Later, another improved decoding algorithm based on SC, named successive cancellation stack (SCS) decoding, is proposed with the explicit aim of increasing the throughput and reducing the latency [36]. The SCS decoder has much lower computational complexity than SCL but it comes at the price of much larger space complexity and will fail to work when the stack is too small. Recently, a low complexity soft-output version of the SC decoder, called soft cancellation (SCAN) decoder, was proposed in [30]. Compared to the BP decoder, the SCAN decoder has much lower computational complexity and requires less memory to store the soft messages. Despite the wide work that has been done in the area of polar decoding in recent years, where none provide the soft outputs with low complexity that would be re-

quired by many practical applications of turbo processing, it is still an open issue to 
find a decoding algorithm with both good error rate performance and low complexity.

Arikan originally described the SC decoding algorithm in the likelihood ratio (LR) domain, and although mathematically simple, operation in this domain is numerically instable, particularly when limited precision arithmetic is used. Instead the SC decoder is usually implemented using the sum-product (SP) algorithm, which operates in the log likelihood ratio (LLR) domain. The SP algorithm achieves optimal decoding performance, but the algorithm requires the evaluation of a large number exponentials and logarithms, which are computationally expensive. The minimum-sum (MS) algorithm is a suboptimal low-complexity alternative to the SP algorithm where the update rules are replace by minimum comparisons and summation operations.

In this chapter, we aim to reduce the decoding complexity of polar codes by applying the normalized minimum-sum (NMS) decoding algorithm, which is a modified version of SP algorithm as demonstrated in [38]. The NMS algorithm provides a compromise between the SP and the MS algorithms with performance similar to the former and complexity close to the latter. The MS messages are modified by scaling the check node messages, so as to enhance the reliability of the propagated messages and to increase the decoding accuracy $[39,40]$.

Since the scaling factor of the normalization is not fixed as it depends on polar code length and rate, the optimal value for this factor can selected by simulation beforehand the optimized implementation by density evolution (DE). DE, proposed by Richardson in [41], is to keep track of extrinsic information density for the messages in the decoding process. Chung [42] presented a threshold estimation of LDPC SP decoding over AWGN in order to approximate the LLRs density function via the symmetry property of the messages. Their idea is to approximate the density of messages (expressed as LLR values) by a Gaussian distribution. And by using the symmetry property of the messages, the variance of Gaussian distributed messages is found to be equal to twice the mean and then it is able to further simplify the analysis by using a single parameter, either the mean or the variance of the message density, to track down the probabilistic evolution. In [43] the factor is calculated as a ratio of expectation value of LLR and found that the performance of the MS algorithm could approach that of the SP algorithm, and thus in this chapter we prove that the optimal scaling factor is the one that the highest noise (lowest SNR) threshold level and the maximum scaling gain equals difference of threshold values. 


\subsection{Reduced-Complexity Check Node Calcula- tions}

The algorithm presented in Section 2.6 relies heavily on the computation of complex nonlinear functions that require a lot of computational power. If one is willing to accept a little degradation in the performance in exchange for smaller delays and less complexity, then the resulting simplification can make the design easier and more tenable to hardware implementation.

At the heart of the SC decoding algorithm is the boxplus operation, which combines two input LLRs, $\lambda_{0}$ and $\lambda_{1}$, to produce one output LLR, $\lambda_{2}$, according to

$$
\lambda_{2}=\lambda_{0} \boxplus \lambda_{1}=\ln \frac{e^{\lambda_{0}} e^{\lambda_{1}}+1}{e^{\lambda_{0}}+e^{\lambda_{1}}} .
$$

Because this calculation must be performed $\frac{N}{2} \log _{2} N$ times to decode a codeword of length $N$, and because it involves evaluating one logarithm and two exponentials (which in turn are difficult to evaluate), evaluation of the boxplus operator is the most resource-intensive component of the SC decoder. Reducing the complexity of the calculation of the boxplus operator is the primary focus of this chapter.

In addition to the complexity issue, direct calculation of (3.1) is numerically unstable when finite precision variables are used. For example, mathematically we expect $\lambda_{2} \rightarrow \lambda_{1}$ as $\lambda_{0} \rightarrow \infty$, but because $e^{\lambda_{0}}$ overflows a finite precision variable even for moderately large $\lambda_{0}$, the value calculated by (3.1) is undefined. To address this issue, (2.16) is an alternative but mathematically equivalent form of (3.1) and is often recommended:

$$
\lambda_{2}=2 \tanh ^{-1}\left(\tanh \frac{\lambda_{0}}{2} \tanh \frac{\lambda_{1}}{2}\right) .
$$

Although this form is roughly equivalent to (3.1) in terms of computational complexity (three nonlinear functions must be evaluated in both cases), (3.2) is more stable [44]. Whereas (3.1) fails if either $\lambda_{0}$ or $\lambda_{1}$ is large, (3.2) only fails if both are large.

There are many other forms for expressing the boxplus operator that are more stable, and in the following we derive a form that is both stable and is helpful for motivating the following discussion on suboptimal reduced-complexity forms. 
By Jacobian logarithm, where

$$
\begin{aligned}
\ln \left(e^{A}+e^{B}\right) & = \begin{cases}A+\ln \left(1+e^{B-A}\right) & \text { if } A \geqslant B \\
B+\ln \left(1+e^{A-B}\right) & \text { otherwise }\end{cases} \\
& =\max (A, B)+\ln \left(1+e^{-|A-B|}\right),
\end{aligned}
$$

we have

$$
\ln \left(e^{\lambda_{0}}+e^{\lambda_{1}}\right)=\max \left(\lambda_{0}, \lambda_{1}\right)+\ln \left(1+e^{-\left|\lambda_{0}-\lambda_{1}\right|}\right),
$$

and

$$
\begin{aligned}
\ln \left(e^{\lambda_{0}} e^{\lambda_{1}}+1\right) & =\ln \left(e^{\lambda_{0}+\lambda_{1}}+e^{0}\right) \\
& =\max \left(\lambda_{0}+\lambda_{1}, 0\right)+\ln \left(1+e^{-\left|\lambda_{0}+\lambda_{1}\right|}\right),
\end{aligned}
$$

so

$$
\begin{aligned}
\lambda_{2} & =\ln \left(e^{\lambda_{0}} e^{\lambda_{1}}+1\right)-\ln \left(e^{\lambda_{0}}+e^{\lambda_{1}}\right) \\
& =\max \left(\lambda_{0}+\lambda_{1}, 0\right)+\ln \left(1+e^{-\left|\lambda_{0}+\lambda_{1}\right|}\right)-\max \left(\lambda_{0}, \lambda_{1}\right)-\ln \left(1+e^{-\left|\lambda_{0}-\lambda_{1}\right|}\right) \\
& =\max \left(\lambda_{0}+\lambda_{1}, 0\right)-\max \left(\lambda_{0}, \lambda_{1}\right)+\ln \frac{1+e^{-\left|\lambda_{0}+\lambda_{1}\right|}}{1+e^{-\left|\lambda_{0}-\lambda_{1}\right|}} .
\end{aligned}
$$

Let $C=\max (A+B, 0)-\max (A, B)$ and consider the following four cases (see Fig. 3.1).

1. $A+B \geqslant 0$ and $A \geqslant B$.

This implies $A \geqslant 0$ and $|A| \geqslant|B|$, along with $C$ where $C=A+B-A=B=\operatorname{sgn}(B)|B|=\operatorname{sgn}(A) \operatorname{sgn}(B)|B|$.

2. $A+B \geqslant 0$ and $A \leqslant B$.

This implies $B \geqslant 0$ and $|A| \leqslant|B|$, along with $C$ where $C=A+B-B=A=\operatorname{sgn}(A)|A|=\operatorname{sgn}(B) \operatorname{sgn}(A)|A|$.

3. $A+B \leqslant 0$ and $A \geqslant B$.

This implies $B \leqslant 0$ and $|A| \leqslant|B|$, along with $C$ where 


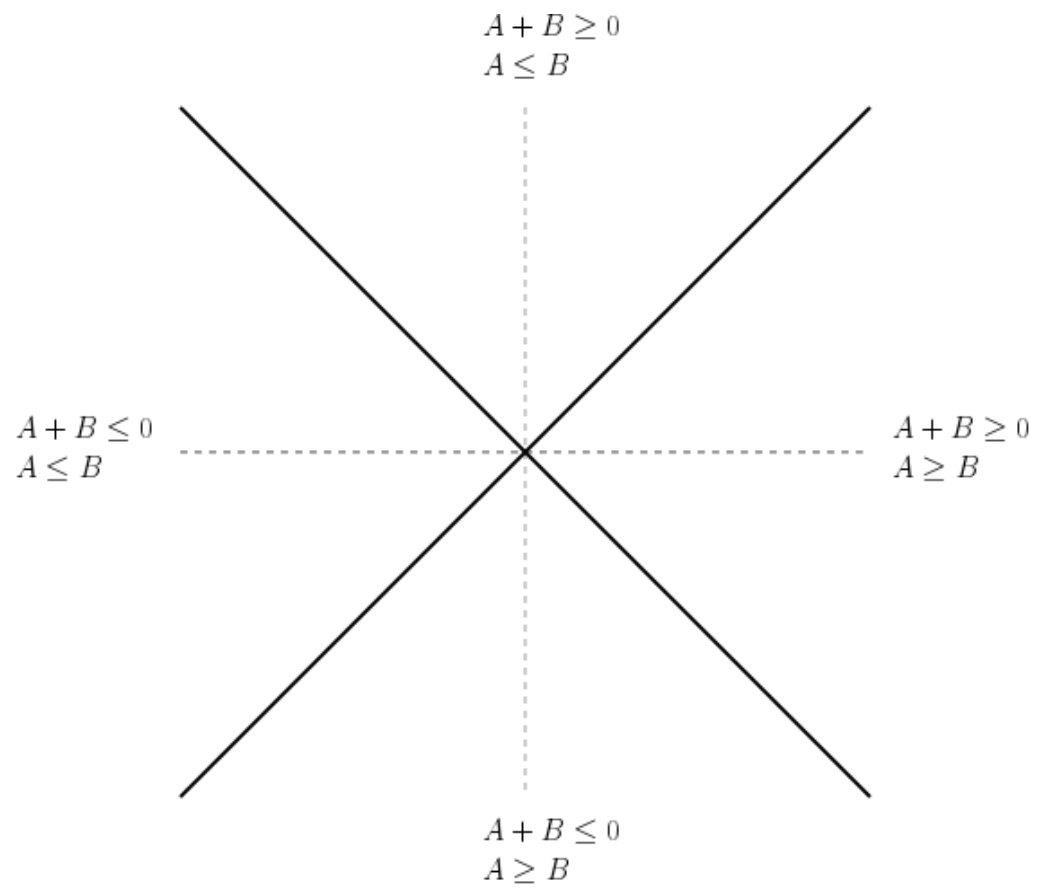

Figure 3.1: LLR decisions.

$C=0-A=-A=-\operatorname{sgn}(A)|A|=\operatorname{sgn}(B) \operatorname{sgn}(A)|A|$.

4. $A+B \leqslant 0$ and $A \leqslant B$.

This implies $A \leqslant 0$ and $|A| \geqslant|B|$, along with $C$ where $C=0-B=-B=-\operatorname{sgn}(B)|B|=\operatorname{sgn}(A) \operatorname{sgn}(B)|B|$.

Since $B \leqslant A$ in cases 1 and 4 , and $A \leqslant B$ in cases 2 and 3 , we can combine all cases as

$$
C=\operatorname{sgn}(A) \operatorname{sgn}(B) \cdot \min (|A|,|B|)
$$

Using (3.7), we can express $\lambda_{2}$ as

$$
\lambda_{2}=\operatorname{sgn}\left(\lambda_{0}\right) \operatorname{sgn}\left(\lambda_{1}\right) \cdot \min \left(\left|\lambda_{0}\right|,\left|\lambda_{1}\right|\right)+\ln \frac{1+e^{-\left|\lambda_{0}+\lambda_{1}\right|}}{1+e^{-\left|\lambda_{0}-\lambda_{1}\right|}},
$$

Although (3.8) is mathematically equivalent to (3.1) and (3.2), it is numerically stable in that it always produces approximately correct results when finite-precision arithmetic is used, regardless of the size of $\lambda_{0}$ and $\lambda_{1}$. However, the complexity in calculating (3.8) is still high (even slightly higher than (3.1)).

We note that (3.1), (3.2) and (3.8) are mathematically "exact". In the context of 
decoding LDPC codes, where the boxplus operator is an essential component of the check node update rule in the BP decoding algorithm, the BP algorithm with an exact implementation of the boxplus operator is referred to as the SP algorithm. Because most of the prior research into reduced complexity implementations of the boxplus operator been done in the context of LDPC codes, and to facilitate relating our work to that research, we keep the same nomenclature and refer to any implementation of SC decoding of polar codes using an exact form of the boxplus operator as SP decoding.

In the literature on LDPC codes, reducing the complexity of the boxplus operator involves using suboptimal inexact implementations. The three main techniques are known as the MS algorithm, the normalized minimum-sum (NMS) algorithm, and the corrected minimum-sum algorithm. We present these techniques in the following.

\subsubsection{Minimum-Sum (MS) Algorithm}

Suppose the LLR produced by the SP algorithm is $\lambda_{2|| \mathrm{SP}}$, then (3.8) can be rewritten as

$$
\lambda_{2|| \mathrm{SP}}=\operatorname{sgn}\left(\lambda_{0} \lambda_{1}\right) \cdot \min \left(\left|\lambda_{0}\right|,\left|\lambda_{1}\right|\right)+r\left(\lambda_{0}, \lambda_{1}\right),
$$

where

$$
r\left(\lambda_{0}, \lambda_{1}\right)=\ln \frac{1+e^{-\left|\lambda_{0}+\lambda_{1}\right|}}{1+e^{-\left|\lambda_{0}-\lambda_{1}\right|}},
$$

is referred to as the correction term.

To reduce the complexity of the check node calculations, the MS algorithm simply neglects the correction term, instead calculating

$$
\lambda_{2|| \mathrm{MS}}=\operatorname{sgn}\left(\lambda_{0} \lambda_{1}\right) \cdot \min \left(\left|\lambda_{0}\right|,\left|\lambda_{1}\right|\right),
$$

but this comes at the expense of a reduction in the performance of the decoder. The correction term has a non-negligible effect on the performance of the decoder [44,45], so it is worthwhile to investigate some of the properties of this term.

Lemma 2. According to (3.10), we have $\left|r\left(\lambda_{0}, \lambda_{1}\right)\right|<\ln 2$ and $\operatorname{sgn}\left(r\left(\lambda_{0}, \lambda_{1}\right)\right)=$ $-\operatorname{sgn}\left(\lambda_{0} \lambda_{1}\right)$ for $\lambda_{0} \lambda_{1} \neq 0$.

Proof. When $\lambda_{0} \lambda_{1}=0$ we have $r\left(\lambda_{0}, \lambda_{1}\right)=0$. When $\lambda_{0}$ and $\lambda_{1}$ are nonzero and have the same sign such that $\lambda_{0} \lambda_{1}>0$ then $\left|\lambda_{0}+\lambda_{1}\right|>\left|\lambda_{0}-\lambda_{1}\right|$, so $r\left(\lambda_{0}, \lambda_{1}\right)<0$. If we 
assume $0<\lambda_{1} \leqslant \lambda_{0}$, then

$$
\begin{aligned}
r\left(\lambda_{0}, \lambda_{1}\right) & =\ln \frac{1+e^{-\lambda_{0}-\lambda_{1}}}{1+e^{-\lambda_{0}+\lambda_{1}}} \\
& =\ln \frac{e^{\lambda_{0}}+e^{-\lambda_{1}}}{e^{\lambda_{0}}+e^{\lambda_{1}}} \\
& \geqslant \ln \frac{e^{\lambda_{0}}+e^{-\lambda_{0}}}{e^{\lambda_{0}}+e^{\lambda_{0}}} \\
& >\ln \frac{1}{2}=-\ln 2 .
\end{aligned}
$$

Thus, $-\ln 2<r\left(\lambda_{0}, \lambda_{1}\right)<0$. Similarly, $r\left(\lambda_{0}, \lambda_{1}\right)>0$ and $0<r\left(\lambda_{0}, \lambda_{1}\right)<\ln 2$ when $\lambda_{0} \lambda_{1}<0$.

\subsubsection{Normalized Minimum-Sum (NMS) Algorithm}

The LLRs produced by the MS algorithm have the same sign as those produced by the $\mathrm{SP}$ algorithm (i.e. $\left.\operatorname{sgn}\left(\lambda_{2|| \mathrm{MS}}\right)=\operatorname{sgn}\left(\lambda_{2|| \mathrm{SP}}\right)\right)$, the MS algorithm overestimates the magnitude (i.e. $\left.\left|\lambda_{2|| \mathrm{MS}}\right| \geqslant\left|\lambda_{2|| \mathrm{SP}}\right|\right)$.

To get performance closer to the SP algorithm, Chen et al. [38] suggested attenuating the magnitude of the MS LLRs, resulting in what is known as the normalized minimum-sum (NMS) algorithm. The check node LLRs are given by

$$
\lambda_{2|| \mathrm{NMS}}=c \lambda_{2|| \mathrm{MS}},
$$

for some scaling factor $c, 0<c \leqslant 1$. To determine the appropriate scaling factor, in [38] the idea of forcing the mean of $\left|\lambda_{2|| \mathrm{NMS}}\right|$ to be equal to the mean of $\left|\lambda_{2|| \mathrm{SP}}\right|$. That is,

$$
c=\frac{\mathrm{E}\left[\left|\lambda_{2|| \mathrm{SP}}\right|\right]}{\mathrm{E}\left[\left|\lambda_{2|| \mathrm{MS}}\right|\right]} .
$$

\subsubsection{Corrected Minimum-Sum Algorithm}

An alternative method is introduced in order to reduce the complexity of the SP algorithm by finding a simple approximation for the correction term. This can be done by approximating the $\ln \left(1+e^{-|\lambda|}\right)$ operations, for which results lie in the interval 
$(0, \ln 2]$. The following two-piece linear approximation of [46] is often used

$$
\begin{aligned}
\ln \left(1+e^{-|\lambda|}\right) & \cong \begin{cases}\ln 2-0.5|\lambda| & \text { for }|\lambda|<2 \ln 2 \\
0 & \text { otherwise }\end{cases} \\
& =\max (\ln 2-0.5|\lambda|, 0)
\end{aligned}
$$

Using this approximation, the check node update is approximated by

$$
\lambda_{2|| \mathrm{MS}+r}=\operatorname{sgn}\left(\lambda_{0} \lambda_{1}\right) \cdot \min \left(\left|\lambda_{0}\right|,\left|\lambda_{1}\right|\right)+\tilde{r}\left(\lambda_{0}, \lambda_{1}\right),
$$

where

$$
\tilde{r}\left(\lambda_{0}, \lambda_{1}\right)=\max \left(\ln 2-0.5\left|\lambda_{0}+\lambda_{1}\right|, 0\right)-\max \left(\ln 2-0.5\left|\lambda_{0}-\lambda_{1}\right|, 0\right)
$$

is the approximation of the exact correction term, $r\left(\lambda_{0}, \lambda_{1}\right)$, given by (3.10).

\subsection{Corrected Normalized Minimum-Sum Algo- rithm}

Although the three techniques offer reduced complexity compared to the SP algorithm, we will show later in this chapter that they leave a gap in the probability of error performance when used in SC decoding of polar codes. Certainly the gap is reduced when NMS or corrected MS is used instead of just MS, but a noticeable gap remains. To help close this gap, we propose the corrected NMS algorithm, which is simply a combination of corrected MS and NMS. The check node uses

$$
\begin{aligned}
\lambda_{2|| \mathrm{N}(\mathrm{MS}+r)} & =c \lambda_{2|| \mathrm{MS}+r} \\
& =c\left[\operatorname{sgn}\left(\lambda_{0} \lambda_{1}\right) \cdot \min \left(\left|\lambda_{0}\right|,\left|\lambda_{1}\right|\right)+\tilde{r}\left(\lambda_{0}, \lambda_{1}\right)\right],
\end{aligned}
$$

where $\tilde{r}\left(\lambda_{0}, \lambda_{1}\right)$ is given by (3.16). In the following we extend the technique of Chen et al. for calculating the scaling factor, $c$, proposed for corrected MS [38], to corrected NMS. Later, we explore the use of density evolution as an alternate method to find the best scaling factor. 


\subsubsection{Normalization Term Calculation Using Rate of Means}

By extending the approach of [38], we force the mean of $\left|c \lambda_{2|| \mathrm{MS}+r}\right|$. For convenience, let $\tilde{\lambda}_{2}=\lambda_{2 \| \mathrm{MS}+r}$ and $\lambda_{2}=\lambda_{2|| \mathrm{SP}}$. The scaling factor is then given by

$$
c=\frac{\mathrm{E}\left[\left|\lambda_{2}\right|\right]}{\mathrm{E}\left[\left|\tilde{\lambda}_{2}\right|\right]}
$$

where

$$
\mathrm{E}\left[\left|\lambda_{2}\right|\right]=2 \mathrm{E}\left[\left|\tanh ^{-1}\left(\tanh \frac{\left|\lambda_{0}\right|}{2} \tanh \frac{\left|\lambda_{1}\right|}{2}\right)\right|\right]
$$

and

$$
\begin{aligned}
\mathrm{E}\left[\left|\tilde{\lambda}_{2}\right|\right]=\mathrm{E}[\mid & \operatorname{sgn}\left(\lambda_{0}\right) \operatorname{sgn}\left(\lambda_{1}\right) \cdot \min \left(\left|\lambda_{0}\right|,\left|\lambda_{1}\right|\right) \\
& +\max \left(\ln 2-0.5\left|\lambda_{0}+\lambda_{1}\right|, 0\right) \\
& \left.-\max \left(\ln 2-0.5\left|\lambda_{0}-\lambda_{1}\right|, 0\right) \mid\right] .
\end{aligned}
$$

Although there are no known closed form expressions for $E\left[\left|\lambda_{2}\right|\right]$ and $E\left[\left|\tilde{\lambda}_{2}\right|\right]$, in the following we derive simple expressions that are readily evaluated numerically.

To calculate $\mathrm{E}\left[\left|\lambda_{2}\right|\right]$, we can rewrite $\lambda_{2}$ as

$$
\begin{aligned}
\lambda_{2} & =2 \tanh ^{-1}\left(\tanh \frac{\lambda_{0}}{2} \tanh \frac{\lambda_{1}}{2}\right) \\
& =\ln \frac{1+\tanh \frac{\lambda_{0}}{2} \tanh \frac{\lambda_{1}}{2}}{1-\tanh \frac{\lambda_{0}}{2} \tanh \frac{\lambda_{1}}{2}}
\end{aligned}
$$

By using Taylor series expansion of $\ln \frac{1+x}{1-x}$ we get

$$
\lambda_{2}=\sum_{n=1}^{\infty} \frac{2}{2 n-1}\left(\tanh \frac{\lambda_{0}}{2} \tanh \frac{\lambda_{1}}{2}\right)^{2 n-1} .
$$

Let

$$
g\left(\lambda_{0}, \lambda_{1}\right)=\left|\lambda_{2}\right|=\left|\sum_{n=1}^{\infty} \frac{2}{2 n-1}\left(\tanh \frac{\lambda_{0}}{2} \tanh \frac{\lambda_{1}}{2}\right)^{2 n-1}\right|
$$


so

$$
\begin{aligned}
\mathrm{E}\left[\left|\lambda_{2}\right|\right]= & \mathrm{E}\left[g\left(\lambda_{0}, \lambda_{1}\right)\right] \\
= & \int_{-\infty}^{\infty} \int_{-\infty}^{\infty} g\left(\lambda_{0}, \lambda_{1}\right) f_{\lambda_{0}, \lambda_{1}}\left(\lambda_{0}, \lambda_{1}\right) \mathrm{d} \lambda_{1} \mathrm{~d} \lambda_{0} \\
= & \int_{0}^{\infty} \int_{0}^{\infty} g\left(\lambda_{0}, \lambda_{1}\right) f_{\lambda_{0}, \lambda_{1}}\left(\lambda_{0}, \lambda_{1}\right) \mathrm{d} \lambda_{1} \mathrm{~d} \lambda_{0} \\
& +\int_{0}^{\infty} \int_{0}^{\infty} g\left(\lambda_{0},-\lambda_{1}\right) f_{\lambda_{0}, \lambda_{1}}\left(\lambda_{0},-\lambda_{1}\right) \mathrm{d} \lambda_{1} \mathrm{~d} \lambda_{0} \\
& +\int_{0}^{\infty} \int_{0}^{\infty} g\left(-\lambda_{0}, \lambda_{1}\right) f_{\lambda_{0}, \lambda_{1}}\left(-\lambda_{0}, \lambda_{1}\right) \mathrm{d} \lambda_{1} \mathrm{~d} \lambda_{0} \\
& +\int_{0}^{\infty} \int_{0}^{\infty} g\left(-\lambda_{0},-\lambda_{1}\right) f_{\lambda_{0}, \lambda_{1}}\left(-\lambda_{0},-\lambda_{1}\right) \mathrm{d} \lambda_{1} \mathrm{~d} \lambda_{0} .
\end{aligned}
$$

We note that

$$
g\left(\lambda_{0}, \lambda_{1}\right)=g\left(\lambda_{0},-\lambda_{1}\right)=g\left(-\lambda_{0}, \lambda_{1}\right)=g\left(-\lambda_{0},-\lambda_{1}\right)
$$

and since $\lambda_{0}$ and $\lambda_{1}$ are i.i.d, with same $\operatorname{PDF} f_{\lambda}(\lambda)$, we have

$$
\mathrm{E}\left[\left|\lambda_{2}\right|\right]=\int_{0}^{\infty} \int_{0}^{\infty} g\left(\lambda_{0}, \lambda_{1}\right)\left[f_{\lambda}\left(\lambda_{0}\right)+f_{\lambda}\left(-\lambda_{0}\right)\right]\left[f_{\lambda}\left(\lambda_{1}\right)+f_{\lambda}\left(-\lambda_{1}\right)\right] \mathrm{d} \lambda_{1} \mathrm{~d} \lambda_{0}
$$

For $\lambda_{0}, \lambda_{1} \geqslant 0$ the absolute value in $(3.22)$ is redundant, so

$$
\begin{aligned}
\mathrm{E}\left[\left|\lambda_{2}\right|\right]= & \int_{0}^{\infty} \int_{0}^{\infty} \sum_{n=1}^{\infty} \frac{2}{2 n-1}\left(\tanh \frac{\lambda_{0}}{2}\right)^{2 n-1}\left(\tanh \frac{\lambda_{1}}{2}\right)^{2 n-1} \\
& \times\left[f_{\lambda}\left(\lambda_{0}\right)+f_{\lambda}\left(-\lambda_{0}\right)\right]\left[f_{\lambda}\left(\lambda_{1}\right)+f_{\lambda}\left(-\lambda_{1}\right)\right] \mathrm{d} \lambda_{1} \mathrm{~d} \lambda_{0} \\
= & \sum_{n=1}^{\infty} \frac{2}{2 n-1}\left[\int_{0}^{\infty}\left(\tanh \frac{\lambda}{2}\right)^{2 n-1}\left(f_{\lambda}(\lambda)+f_{\lambda}(-\lambda)\right) \mathrm{d} \lambda\right]^{2}
\end{aligned}
$$

To find an expression for $f_{\lambda}(\lambda)$, suppose the $x \in\{ \pm 1\}$ with equal a prior probability is transmitted over an AWGN channel with double-sided noise power spectral density $\sigma^{2}=\frac{N_{o}}{2}$. The received sample, $y$, has a Gaussian likelihood function given $x$ transmitted of

$$
f_{Y}(y \mid x)=\frac{1}{\sqrt{2 \pi \sigma^{2}}} e^{-\frac{(y-x)^{2}}{2 \sigma^{2}}}
$$


and the PDF of $y$ is

$$
f_{Y}(y)=\frac{1}{2} f_{Y}(y \mid x=-1)+\frac{1}{2} f_{Y}(y \mid x=+1) .
$$

The calculated LLR of the channel output is

$$
\begin{aligned}
\lambda & =\ln \frac{\operatorname{Pr}\{y \mid x=+1\}}{\operatorname{Pr}\{y \mid x=-1\}} \\
& =\ln \frac{\frac{1}{\sqrt{2 \pi \sigma^{2}}} e^{-\frac{(y-1)^{2}}{2 \sigma^{2}}}}{\frac{1}{\sqrt{2 \pi \sigma^{2}}} e^{-\frac{(y+1)^{2}}{2 \sigma^{2}}}} \\
& =\ln \left(e^{\frac{4 y}{2 \sigma^{2}}}\right) \\
& =\frac{2 y}{\sigma^{2}} .
\end{aligned}
$$

The PDF of $\lambda$ is then

$$
\begin{aligned}
f_{\lambda}(\lambda) & =\frac{\sigma^{2}}{2} f_{Y}\left(\frac{\sigma^{2}}{2} \lambda\right) \\
& =\frac{\sigma^{2}}{4} f_{Y}\left(\frac{\sigma^{2}}{2} \lambda \mid x=-1\right)+\frac{\sigma^{2}}{4} f_{Y}\left(\frac{\sigma^{2}}{2} \lambda \mid x=+1\right) .
\end{aligned}
$$

Because $f_{Y}(y \mid x=+1)=f_{Y}(-y \mid x=-1)$, we also have $f_{\lambda}(\lambda)=f_{\lambda}(-\lambda)$, so

$$
f_{\lambda}(\lambda)+f_{\lambda}(-\lambda)=2 f_{\lambda}(\lambda) .
$$

As a result

$$
\mathrm{E}\left[\left|\lambda_{2}\right|\right]=\sum_{n=1}^{\infty} \frac{8}{2 n-1}\left[\int_{0}^{\infty}\left(\tanh \frac{\lambda}{2}\right)^{2 n-1} f_{\lambda}(\lambda) \mathrm{d} \lambda\right]^{2},
$$

where $f_{\lambda}(\lambda)$ is given by (3.30). We note that only the first few terms of the summation are needed for accurate results, and the integral is easily evaluated numerically. Furthermore $\mathrm{E}\left[\left|\lambda_{2}\right|\right]$ is a function of $\sigma^{2}$. 
To calculate $\mathrm{E}\left[\left|\tilde{\lambda}_{2}\right|\right]$, we define $\lambda_{a}$ as

$$
\begin{aligned}
\lambda_{a} & =\min \left(\left|\lambda_{0}\right|,\left|\lambda_{1}\right|\right) \\
& =\min \left(W_{0}, W_{1}\right),
\end{aligned}
$$

where $W_{n}=\left|\lambda_{n}\right|$ for $n \in\{0,1\}$ is defined for convenience. Since $W_{0}$ and $W_{1}$ are i.i.d., with PDF $f_{W}(w)=2 f_{\lambda}(w)$ and $f_{\lambda}(\lambda)$ is given by (3.30), the CDF of $\lambda_{a}$ is

$$
\begin{array}{rlr}
F_{\lambda_{a}}\left(\ell_{a}\right) & =F_{W_{0}}\left(\ell_{a}\right)+F_{W_{1}}\left(\ell_{a}\right)-F_{W_{0}}\left(\ell_{a}\right) F_{W_{1}}\left(\ell_{a}\right) \\
& =2 F_{W_{0}}\left(\ell_{a}\right)-F_{W_{0}}^{2}\left(\ell_{a}\right) & \\
& =1-\left(1-F_{W_{0}}\left(\ell_{a}\right)\right)^{2} & \\
& =1-\operatorname{Pr}\left\{\lambda_{a}>\ell_{a}\right\} & \text { (by definition) }
\end{array}
$$

Hence

$$
\operatorname{Pr}\left\{\lambda_{a}>\ell_{a}\right\}=\left(1-F_{W_{0}}\left(\ell_{a}\right)\right)^{2} .
$$


It follows with $\mathrm{Q}(x)=\frac{1}{\sqrt{2 \pi}} \int_{x}^{\infty} e^{-\frac{u^{2}}{2}} \mathrm{~d} u$ that

$$
\begin{aligned}
\mathrm{E}\left[\lambda_{a}\right]= & \int_{0}^{\infty} \operatorname{Pr}\left\{\lambda_{a}>\ell_{a}\right\} \mathrm{d} \ell_{a} \quad\left(\text { since } \lambda_{a}>0\right) \\
= & \int_{0}^{\infty}\left(1-F_{W_{0}}\left(\ell_{a}\right)\right)^{2} \mathrm{~d} \ell_{a} \\
= & \int_{0}^{\infty}\left[1-\left(1-\mathrm{Q}\left(\frac{\ell_{a}+\frac{2}{\sigma^{2}}}{\frac{2}{\sigma}}\right)-\mathrm{Q}\left(\frac{\ell_{a}-\frac{2}{\sigma^{2}}}{\frac{2}{\sigma}}\right)\right)\right]^{2} \mathrm{~d} \ell_{a}{ }^{1} \\
= & \int_{0}^{\infty}\left[\mathrm{Q}\left(\frac{\ell_{a}+\frac{2}{\sigma^{2}}}{\frac{2}{\sigma}}\right)+\mathrm{Q}\left(\frac{\ell_{a}-\frac{2}{\sigma^{2}}}{\frac{2}{\sigma}}\right)\right]^{2} \mathrm{~d} \ell_{a} \\
= & \int_{\frac{2}{\sigma^{2}}}^{\infty}\left[\mathrm{Q}\left(\frac{\ell_{a}+\frac{2}{\sigma^{2}}}{\frac{2}{\sigma}}\right)+\mathrm{Q}\left(\frac{\ell_{a}-\frac{2}{\sigma^{2}}}{\frac{2}{\sigma}}\right)\right]^{2} \mathrm{~d} \ell_{a} \\
& +\int_{0}^{\frac{2}{\sigma^{2}}}\left[\mathrm{Q}\left(\frac{\ell_{a}+\frac{2}{\sigma^{2}}}{\frac{2}{\sigma}}\right)+1-\mathrm{Q}\left(\frac{\frac{2}{\sigma^{2}}-\ell_{a}}{\frac{2}{\sigma}}\right)\right]^{2} \mathrm{~d} \ell_{a} \\
\approx & \int_{0}^{\frac{2}{\sigma^{2}}}\left[\mathrm{Q}\left(\frac{\ell_{a}+\frac{2}{\sigma^{2}}}{\frac{2}{\sigma}}\right)+1-\mathrm{Q}\left(\frac{\frac{2}{\sigma^{2}}-\ell_{a}}{\frac{2}{\sigma}}\right)\right]^{2} \mathrm{~d} \ell_{a} .
\end{aligned}
$$

Again, let

$$
\lambda_{b}=\max \left(\ln 2-0.5\left|\lambda_{0}+\lambda_{1}\right|, 0\right)
$$

and in order to simplify the analysis we are going to assume the following: Let

$$
\stackrel{+}{W}_{2}=\left|\lambda_{0}+\lambda_{1}\right| \quad \text { (i.i.d. r.vs.) }
$$

then its density

$$
f_{W_{2}}(\stackrel{+}{w})=2 f_{\lambda_{0}+\lambda_{1}}(\stackrel{+}{w}) u(\stackrel{+}{w})
$$

where

$$
\lambda_{0}+\lambda_{1} \sim N\left(\frac{2}{\sigma^{2}}, \frac{8}{\sigma^{2}}\right) .
$$

Also, let

$$
\tilde{W}_{2}=\ln 2-0.5 \stackrel{+}{W}_{2}
$$

${ }^{1}$ See Section B.1 for $F_{W_{0}}\left(\ell_{a}\right)=1-\mathrm{Q}\left(\frac{\ell_{a}+\frac{2}{\sigma^{2}}}{\frac{2}{\sigma}}\right)-\mathrm{Q}\left(\frac{\ell_{a}-\frac{2}{\sigma^{2}}}{\frac{2}{\sigma}}\right)$. 
which gives

$$
\begin{aligned}
F_{\tilde{W}_{2}}(\tilde{w}) & =\operatorname{Pr}\left\{\tilde{W}_{2} \leqslant \tilde{w}\right\} \\
& =\operatorname{Pr}\left\{\ln 2-0.5 \stackrel{+}{W}_{2}<\tilde{w}\right\} \\
& =\operatorname{Pr}\left\{\stackrel{+}{W}_{2}>\frac{\ln 2-\tilde{w}}{0.5}\right\} \\
& =1-F_{W_{2}}\left(\frac{\ln 2-\tilde{w}}{0.5}\right) .
\end{aligned}
$$

But $\lambda_{b}=\max \left(\tilde{W}_{2}, 0\right)$, this yields

$$
\begin{array}{rlrl}
F_{\lambda_{b}}\left(\ell_{b}\right) & =F_{o}\left(\ell_{b}\right) F_{\tilde{W}_{2}}\left(\ell_{b}\right) & & \\
& =F_{\widetilde{W}_{2}}\left(\ell_{b}\right) & & F_{o}\left(\ell_{b}\right)=1 \text { for } \ell_{b} \geqslant 0 \text { according } \\
& =1-F_{W_{2}}\left(\frac{\ln 2-\ell_{b}}{0.5}\right) & & \\
& \text { to zero-variance limit theorem }
\end{array}
$$

Hence

$$
\operatorname{Pr}\left\{\ell_{b}>w\right\}=F_{W_{2}}\left(\frac{\ln 2-\ell_{b}}{0.5}\right) .
$$


Therefore

$$
\begin{aligned}
\mathrm{E}\left[\lambda_{b}\right]= & \int_{-\infty}^{\infty} \operatorname{Pr}\left\{\lambda_{b}>\ell_{b}\right\} \mathrm{d} \ell_{b} \\
= & \int_{0}^{\infty} F_{W_{2}}\left(\frac{\ln 2-\ell_{b}}{0.5}\right) \mathrm{d} \ell_{b} \\
= & \int_{0}^{\infty} 1-\mathrm{Q}\left(\frac{\frac{\ln 2-\ell_{b}}{0.5}+\frac{4}{\sigma^{2}}}{\frac{\sqrt{8}}{\sigma}}\right)-\mathrm{Q}\left(\frac{\frac{\ln 2-\ell_{b}}{0.5}-\frac{4}{\sigma^{2}}}{\frac{\sqrt{8}}{\sigma}}\right) \mathrm{d} \ell_{b} \\
= & \int_{0}^{\ln 2-0.5 \frac{4}{\sigma^{2}}} 1-\mathrm{Q}\left(\frac{\frac{\ln 2-\ell_{b}}{0.5}+\frac{4}{\sigma^{2}}}{\frac{\sqrt{8}}{\sigma}}\right)-\mathrm{Q}\left(\frac{\frac{\ln 2-\ell_{b}}{0.5}-\frac{4}{\sigma^{2}}}{\frac{\sqrt{8}}{\sigma}}\right) \mathrm{d} \ell_{b} \\
& +\int_{\ln 2-0.5 \frac{4}{\sigma^{2}}}^{\ln 2+0.5 \frac{4}{\sigma^{2}}} \mathrm{Q}\left(\frac{\frac{4}{\sigma^{2}}-\frac{\ln 2-\ell_{b}}{0.5}}{\frac{\sqrt{8}}{\sigma}}\right)-\mathrm{Q}\left(\frac{\frac{\ln 2-\ell_{b}}{0.5}+\frac{4}{\sigma^{2}}}{\frac{\sqrt{8}}{\sigma}}\right) \mathrm{d} \ell_{b} .
\end{aligned}
$$

Finally, following the similar analysis above in calculating $\mathrm{E}\left[\lambda_{b}\right]$ we can find $\mathrm{E}\left[\lambda_{c}\right]$ by assuming that

$$
\begin{gathered}
\bar{W}_{2}=\left|\lambda_{0}-\lambda_{1}\right| \\
\lambda_{0}-\lambda_{1} \sim N\left(0, \frac{8}{\sigma^{2}}\right) \\
\lambda_{c}=\max \left(\ln 2-0.5\left|\lambda_{0}-\lambda_{1}\right|, 0\right) .
\end{gathered}
$$

Then,

$$
\begin{aligned}
\mathrm{E}\left[\lambda_{c}\right] & =\left.\mathrm{E}\left[\lambda_{b}\right]\right|_{\text {mean }=0, \text { variance }=\frac{8}{\sigma^{2}}} \\
& =\int_{0}^{\ln 2} 1-2 \mathrm{Q}\left(\frac{\ln 2-\ell_{c}}{0.5 \frac{\sqrt{8}}{\sigma}}\right) \mathrm{d} \ell_{c}
\end{aligned}
$$

As a result, the factor $c$ is obtained from (3.18).

Fig. 3.2 depicts the block error rate (BLER) performance with code length $N$ set as 1024 and the code rate $R=0.5$. It shows that the loss relative to the $\mathrm{SP}$ decoder suffered by the MS decoder is about $0.37 \mathrm{~dB}$, while all other reduced complexity 


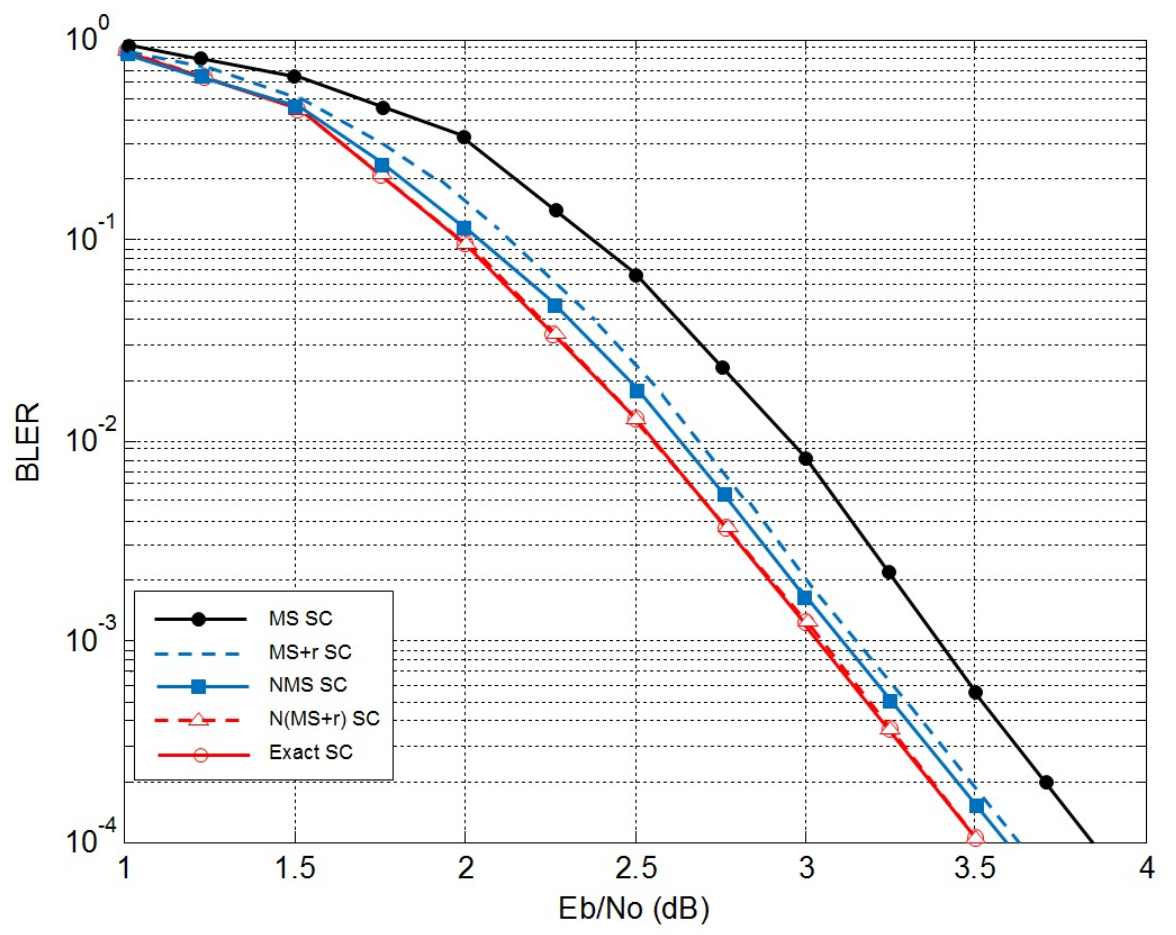

Figure 3.2: BLER performances for decoding algorithms assuming $N=1024 \mathrm{SC}$ polar code with code rate $R=0.5$ and $c=0.869$.

variants of the SP algorithm perform very close to the conventional SP. In particular, the NMS appears no difference for small $E_{b} / N_{0}$ and a barely noticeable degradation $(\approx 0.08 \mathrm{~dB})$ at high $E_{b} / N_{0}$ with respect to SP algorithm. The MS at the presence of $r$ is approximately $0.16 \mathrm{~dB}$ inferior to $\mathrm{SP}$ and when scaling is applied, it outperforms the NMS achieving essentially optimal SP performance.

Fig. 3.3 shows the performance of NMS SC decoder as compared to SP decoder performance under three blocklengths $N=1024,2048,4096$ and $R$ is half over a BAWGN channel. It is shown that applying the correction term with normalization could achieve essentially optimal SP performance, where there is no remarkable performance loss for moderate length codes and is very small (roughly evaluated at 0.15 dB) for longer codes. 


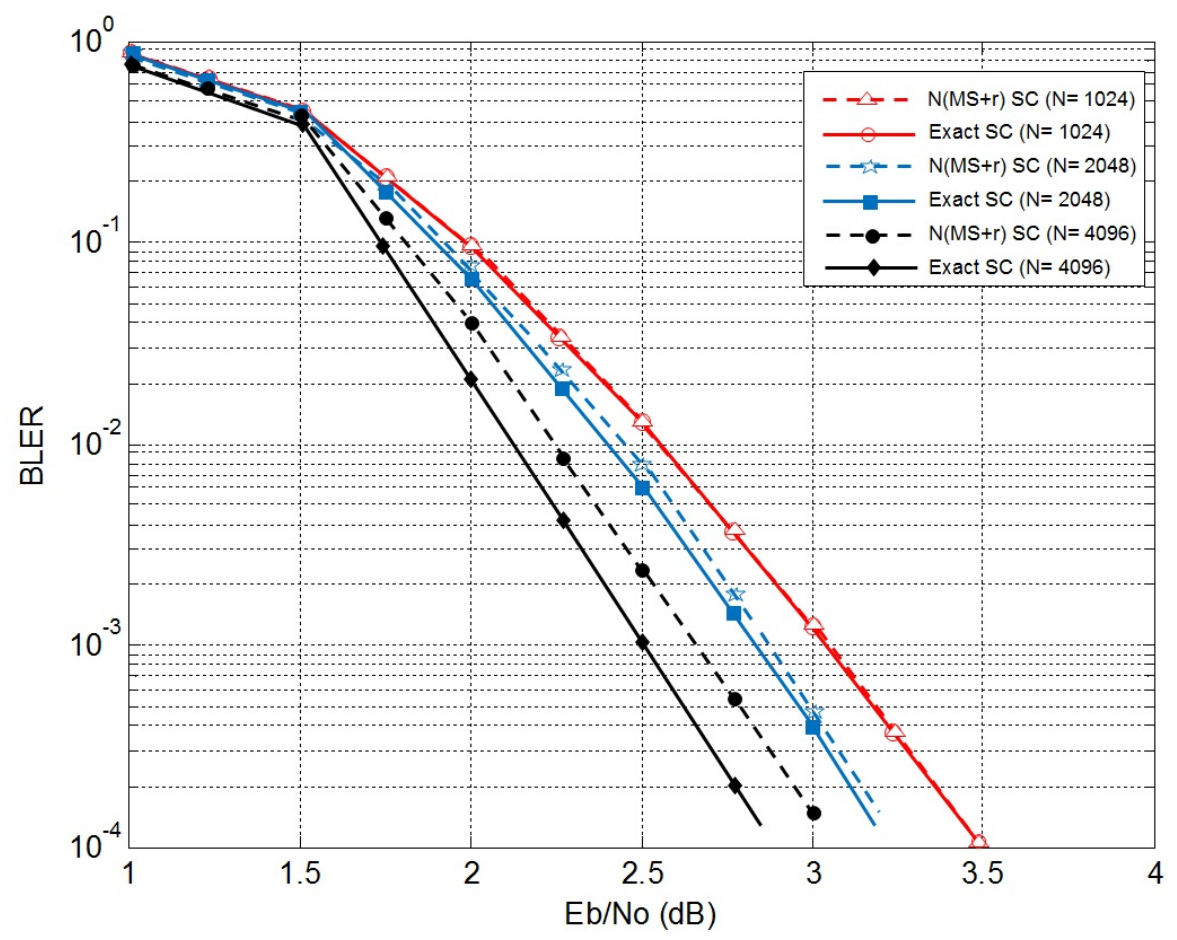

Figure 3.3: SP/NMS SC decoder performances under different blocklengths with $R=0.5$.

\subsubsection{Complexity Discussion}

Although SC-based decoding algorithms have the same time complexity $O\left(N \log _{2} N\right)$, their own hardware realization are different. SC-Arikan algorithm has three hyperbolic core operations as in (2.35) while SC-MS in (3.8) includes nonlinear functions like logarithm and division which have been reduced in NMS-SC in (3.15). This approach exhibited near optimum decoding performance, reduced the complexity and increased robustness against numerical problems. For more details about basic operators and their weights see [47].

\subsection{Performance of NMS-SC Polar Decoding Un- der Density Evolution Method}

Density evolution is an effective numerical method to analyze the performance of message passing iterative decoding algorithms based on graphs. The idea for this method can be traced back to Gallager's work [48]. DE assumed that all messages 
are independent [41]. Clearly, if the $\lambda_{2}$ messages were truly Gaussian, then so would the final $Y$ close to Gaussian. So that to calculate the distribution function $f_{\tilde{\lambda}\left(\ell \mid \mu_{\ell}, \sigma_{\ell}^{2}\right)}$ in an easy way, it is more convenience to derive its variance $\left(\sigma_{\ell}^{2}\right)$ analytically depending on the values of $\mathrm{E}\left[\left|\tilde{\lambda}_{2}\right|\right]$ and $\mathrm{E}\left[\left|\tilde{\lambda}_{2}\right|^{2}\right]$ assuming all $\lambda_{2} \mathrm{~s}$ are independent and Gaussian distributed $[49,50]$.

The derivation is rather lengthy (Section B.2) and leads to the formulas:

$$
\begin{aligned}
\mathrm{E}\left[\left|\tilde{\lambda}_{2}\right|^{2}\right]= & \int_{0}^{\left(\frac{2}{\sigma^{2}}\right)^{2}} 1-4\left[\mathrm{Q}\left(\frac{\mu_{\lambda_{0}}+\sqrt{\ell}}{\sigma_{\lambda_{0}}}\right)-\mathrm{Q}\left(\frac{\mu_{\lambda_{0}}-\sqrt{\ell}}{\sigma_{\lambda_{0}}}\right)\right] \mathrm{d} \ell \\
& +\int_{-2\left(\frac{2}{\sigma^{2}}\right)^{2}}^{0} 1-3\left[\mathrm{Q}\left(\frac{\mu_{\lambda_{0}}+\sqrt{\frac{-\ell}{2}}}{\sigma_{\lambda_{0}}}\right)-\mathrm{Q}\left(\frac{\mu_{\lambda_{0}}-\sqrt{\frac{-\ell}{2}}}{\sigma_{\lambda_{0}}}\right)\right] \mathrm{d} \ell .
\end{aligned}
$$

With $\sigma_{\ell}^{2}=\mathrm{E}\left[\left|\tilde{\lambda}_{2}\right|^{2}\right]-\left(\mathrm{E}\left[\left|\tilde{\lambda}_{2}\right|\right]\right)^{2} \Longrightarrow$

$$
f_{\tilde{\lambda}_{2}}(\ell) \approx \frac{1}{\sqrt{2 \pi} \sigma_{\ell}} e^{\frac{-\left(\ell-\mu_{\ell}\right)^{2}}{2 \sigma_{\ell}^{2}}} .
$$

\subsubsection{Justification of Gaussian Approximation}

To explain why the message densities are approximately Gaussian, we revisit (3.2) again; then the following equivalent update rule is often used:

$$
\begin{aligned}
\lambda_{2} & =2 \tanh ^{-1}\left(\tanh \frac{\lambda_{0}}{2} \tanh \frac{\lambda_{1}}{2}\right) \\
& =\operatorname{sgn}\left(\lambda_{0} \lambda_{1}\right) \cdot 2 \tanh ^{-1}\left(\ln ^{-1}\left(\ln \left(\tanh \left|\frac{\lambda_{0}}{2}\right| \tanh \left|\frac{\lambda_{1}}{2}\right|\right)\right)\right) \\
& =\operatorname{sgn}\left(\lambda_{0} \lambda_{1}\right) \cdot 2 \tanh ^{-1}\left(\ln ^{-1}\left(\ln \left(\tanh \left|\frac{\lambda_{0}}{2}\right|\right)+\ln \left(\tanh \left|\frac{\lambda_{1}}{2}\right|\right)\right)\right) \\
& =\operatorname{sgn}\left(\lambda_{0} \lambda_{1}\right) \cdot f^{-1}\left(f\left(\left|\lambda_{0}\right|\right)+f\left(\left|\lambda_{1}\right|\right)\right) .
\end{aligned}
$$

Where the function $f(|x|)=-\ln \left(\tanh \left(\frac{|x|}{2}\right)\right)=\ln \left(\frac{e^{|x|}+1}{e^{|x|}-1}\right)$ [48] is an involution transform, i.e., has the property $f(f(x))=\ln \left(\frac{e^{f(x)}+1}{e^{f(x)}-1}\right)=x$, and $f(0)=\infty, f(\infty)=$ 0 . 
So, in order to see how this term, $f^{-1}\left(f\left(\left|\lambda_{0}\right|\right)+f\left(\left|\lambda_{1}\right|\right)\right)$, of the LLR messages approximately preserves the Gaussian property, we need to establish a few useful properties of log-normal distributions.

Definition 8. The random variable $X=\ln Y$ has a Gaussian distribution if $Y$ has log-normal distribution. The log-normal PDF for $Y$ can be formulated as

$$
f_{Y}(x)=\frac{1}{\sqrt{2 \pi} x \sigma_{x}} e^{\frac{-\left(\ln x-\mu_{x}\right)^{2}}{2 \sigma_{x}^{2}}} \quad \text { where } x>0 .
$$

Here we have $X \sim N\left(\mu_{x}, \sigma_{x}^{2}\right)$ and $Y \sim L N\left(\mu_{x}, \sigma_{x}^{2}\right)$ with mean $(Y)$ (or $\left.\mu_{y}\right)$ and $\operatorname{variance}(Y)\left(\right.$ or $\left.\sigma_{y}^{2}\right)$ as

$$
\begin{aligned}
\mu_{y} & =e^{\mu_{x}+\frac{1}{2} \sigma_{x}^{2}} \\
\sigma_{y}^{2} & =\mu_{y}^{2}\left(e^{\sigma_{x}^{2}}-1\right) .
\end{aligned}
$$

Lemma 3. If $|x|$ shows a Gaussian distribution, then $f(x)=\ln \left(\frac{e^{|x|}+1}{e^{|x|}-1}\right)$ has approximate log-normal distribution.

Proof. Using Taylor expansion for $e^{|x|}>1$,

$$
\begin{aligned}
f(x) & =\ln \left(\frac{e^{|x|}+1}{e^{|x|}-1}\right) \\
& =2\left[\frac{1}{e^{|x|}}+\frac{1}{3}\left(\frac{1}{e^{|x|}}\right)^{3}+\frac{1}{5}\left(\frac{1}{e^{|x|}}\right)^{5}+\ldots\right] \\
& =2\left[e^{-|x|}+\frac{e^{-3|x|}}{3}+\frac{e^{-5|x|}}{5}+\frac{e^{-7|x|}}{7}+\ldots\right] .
\end{aligned}
$$

We have $|x|$ has approximated Gaussian distribution (Definition 8 where the distribution of the absolute value of a random variable with normal distribution is called folded normal distribution) then by the definition $e^{|x|}$ follows the approximated lognormal distribution. As well, $\beta|x|$ still satisfies Gaussian distribution for $\pm \beta$ integers, so that each term of $f(x), \alpha e^{-\beta|x|}$, also preserves its log-normality with $\alpha \neq 0$. By continuity ${ }^{2}, f(x)$ has approximated log-normal distribution.

\footnotetext{
${ }^{2}$ The distribution of the sum of $n$ independent log-normal variables is approximately log-normal [51-53].
} 
Lemma 4. If $|x|$ shows a log-normal distribution, then $f(x)=\ln \left(\frac{e^{|x|}+1}{e^{|x|}-1}\right)$ has approximate Gaussian distribution.

Proof. This claim looks like the reverse of what stated in Lemma 3, and for this, we are going to use the inverse of $f(x), f^{-1}(x)$. Verify:

$$
f^{-1}(x)=\ln \left(\frac{e^{|x|}+1}{e^{|x|}-1}\right)=f(x) .
$$

We have $f^{-1}(x)=f(x)$, where $f(x)$ shows log-normal distribution to Gaussian messages (Lemma 3), this follows that log-normal distribution messages produce an approximate Gaussian distribution $f(x)$.

\subsubsection{Alternative Proof}

We introduce an alternative proof for the above claim $(f(x)$ has approximate Gaussian Distribution) as the following:

According to the strong law of large numbers (LLN),

$$
\frac{\sigma_{y}}{\mu_{y}} \stackrel{a . s .}{\longrightarrow} 0
$$

then by (3.56), we have

$$
\frac{\mu_{y} \sqrt{e^{\sigma_{x}^{2}}-1}}{\mu_{y}} \longrightarrow 0 \quad \text { or } \quad \sigma_{x} \longrightarrow 0
$$

From $(3.60), \sigma_{x}^{2}=\ln \left(\frac{\sigma_{y}^{2}}{\mu_{y}^{2}}+1\right)$, and due to (3.59) we also have

$$
\sigma_{x}^{2} \longrightarrow \frac{\sigma_{y}^{2}}{\mu_{y}^{2}} \quad \text { or } \quad \sigma_{x} \longrightarrow \frac{\sigma_{y}}{\mu_{y}} .
$$

For any $(\ell)$ in the support of the distribution (set of all numbers whose PDF is strictly positive), we have

$$
\frac{\ell}{\mu_{y}} \stackrel{\text { a.s. }}{\longrightarrow} 1
$$


then by (3.61), this yields

$$
\sigma_{x} \longrightarrow \frac{\sigma_{y}}{\ell} \quad \text { or } \quad \ell \sigma_{x} \longrightarrow \sigma_{y} .
$$

Hence, the first part of $f_{Y}(x)$ will modify to

$$
\sqrt{2 \pi} \ell \sigma_{x} \longrightarrow \sqrt{2 \pi} \sigma_{y}
$$

Now, let

$$
\ell=\mu_{y} \frac{\ell}{\mu_{y}}
$$

then

$$
\ln (\ell)=\ln \left(\mu_{y}\right)+\ln \left(\frac{\ell}{\mu_{y}}\right) .
$$

By (3.62), we have

$$
\ln \left(\frac{\ell}{\mu_{y}}\right) \longrightarrow \frac{\ell}{\mu_{y}}-1=\frac{\ell-\mu_{y}}{\mu_{y}} \cdot 3
$$

From (3.55)

$$
\mu_{x}=\ln \left(\mu_{y}\right)-\frac{1}{2} \sigma_{x}^{2}
$$

and due to (3.60) then

$$
\mu_{x} \longrightarrow \ln \left(\mu_{y}\right) .
$$

Hence, the second part of $f_{Y}(x)$ above will also update as

$$
\begin{array}{cl} 
& \frac{\ln (\ell)-\mu_{x}}{\sigma_{x}}, \\
\stackrel{\text { by (3.66) }}{\longrightarrow} & \frac{\ln \left(\mu_{y}\right)+\ln \left(\frac{\ell}{\mu_{y}}\right)-\mu_{x}}{\sigma_{x}}, \\
\stackrel{\text { by }(3.67,3.69)}{\longrightarrow} & \frac{\mu_{x}+\frac{\ell-\mu_{y}}{\mu_{y}}-\mu_{x}}{\sigma_{x}}=\frac{\ell-\mu_{y}}{\mu_{y} \sigma_{x}}, \\
& \frac{\ell-\mu_{y}}{\sigma_{y}} .
\end{array}
$$

\footnotetext{
${ }^{3}$ See Section B.3 for linear approximation to $\ln x$ at $x \rightarrow 1$.
} 
Finally, by $(3.64,3.70)$ the sum distribution of a very large set of independent lognormal random variables exhibits Gaussian

$$
f_{Y}(y) \approx \frac{1}{\sqrt{2 \pi} \sigma_{y}} e^{\frac{-\left(y-\mu_{y}\right)^{2}}{2 \sigma_{y}^{2}}} .
$$

\subsubsection{Channel Symmetry Condition}

Base Gaussian approximation, the principle of symmetry is the main condition that most DE methods use it [49]. It states that for an LLR density function $f(x)$ then it is satisfied that

$$
f(x)=e^{x} f(-x)
$$

for all messages. Also it is supposed that $\sigma^{2}=2 \mu$ where $\mu$ is the mean and $\sigma^{2}$ is variance [42].

\subsubsection{Optimization Factor, $c$}

According to $(3.18,3.71)$, here we set

$$
c f_{Y}(c y) \Rightarrow f_{Y}(y) \text {. }
$$

It is shown in Fig. 3.4 that the densities are approximately Gaussian distribution. For a given polar code, the performances of the NMS algorithm with different normalization factor $c$ are different. The impact of $c$ on the BLER of different $E_{b} / N_{0}$ is shown in Fig. 3.5. For a particular $E_{b} / N_{0}$, we took the value of that causes the minimum error rate. It can be seen that the optimal amount of scaling required at each $E_{b} / N_{0}$ increases with $E_{b} / N_{0}$. One can also observe that at lower values of $E_{b} / N_{0}$, BLER is less sensitive to $c$ and the optimal value of $c$ is not very sensitive to $E_{b} / N_{0}$.

\subsection{Numerical Results}

Convergence thresholds at a rate of one half polar code with blocklength of 4096 under NMS-SC decoding are given in Table 3.1. For this code, the MNS without narmalization $(c=1)$ loses about $0.4 \mathrm{~dB}$ gain over the standard $\mathrm{SC}$ version. However, 


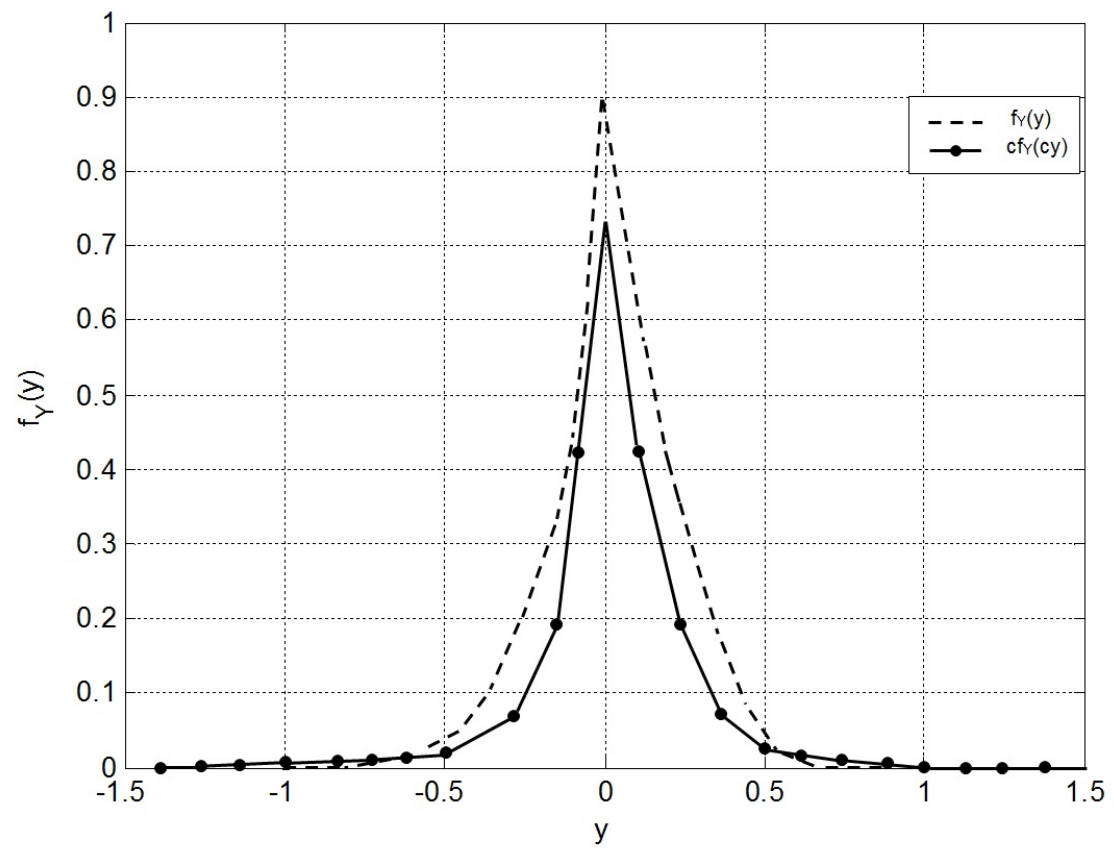

Figure 3.4: Density function before and after normalization with $c=0.787$.

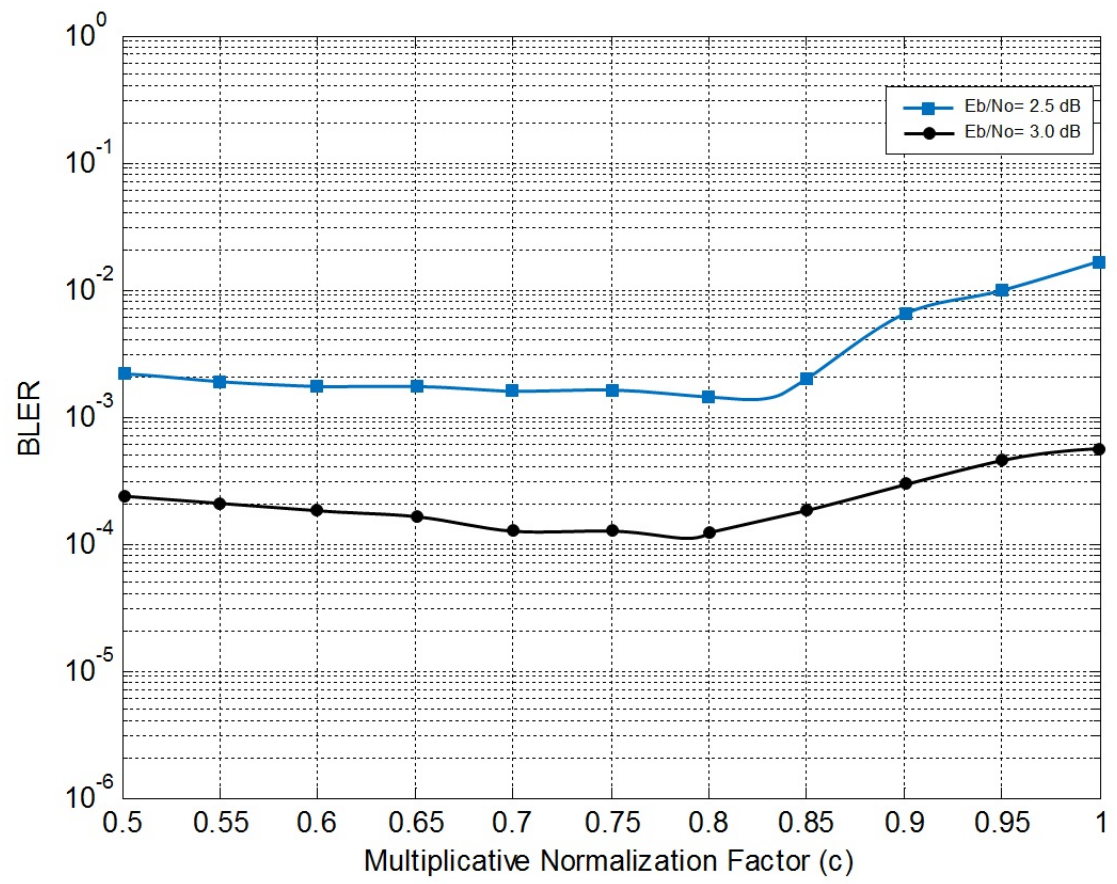

Figure 3.5: Effect of $c$ on BLER for $N=4096$ blocklength polar code decoded by NMS-SC. 
Table 3.1: Threshold values for polar code of length 4096 and rate 0.5 with two normalization factor $c$

\begin{tabular}{cc}
\hline Normalization Factor & $E_{b} / N_{0}(\mathrm{~dB})$ \\
\hline 1.0 & 2.58 \\
0.90 & 2.54 \\
0.787 & 2.20 \\
\hline
\end{tabular}

with the normalized MS algorithm, the gain is reduced to $0.04 \mathrm{~dB}$ when $c=0.787$ as in Fig. 3.5. For comparison, we also plot the performance with $c=0.90$ which is not as good as the latter.

In Fig. 3.6, we have BLER curve for standard SC along with the curves of NMS$\mathrm{SC}$ density evolution technique corresponding to different normalization factor. As mentioned, the threshold depends on $c$. With the optimal $c=0.787$, the threshold is $2.58-2.20=0.38 \mathrm{~dB}$ lower than that of decoding without normalization ( or $c=1)$. This value closes to the performance difference corresponded to the factors 0.787 and 1 as shown in Fig. 3.6.

\subsection{Summary}

We have shown in this chapter that by incorporating a piece-wise linear correcting term to the LLR results through the SC polar decoder process, then the NMS algorithm performance can approach the SP decoder but there is still a noticeable degradation in performance for codes with blocklength moderately long. For this, we adopted the Gaussian approximation by means of DE which could huge help in obtaining the optimal parameters faster and easier, and accordingly get a very close performance with SP decoding algorithm. This can more help in the side of hardware realization. 


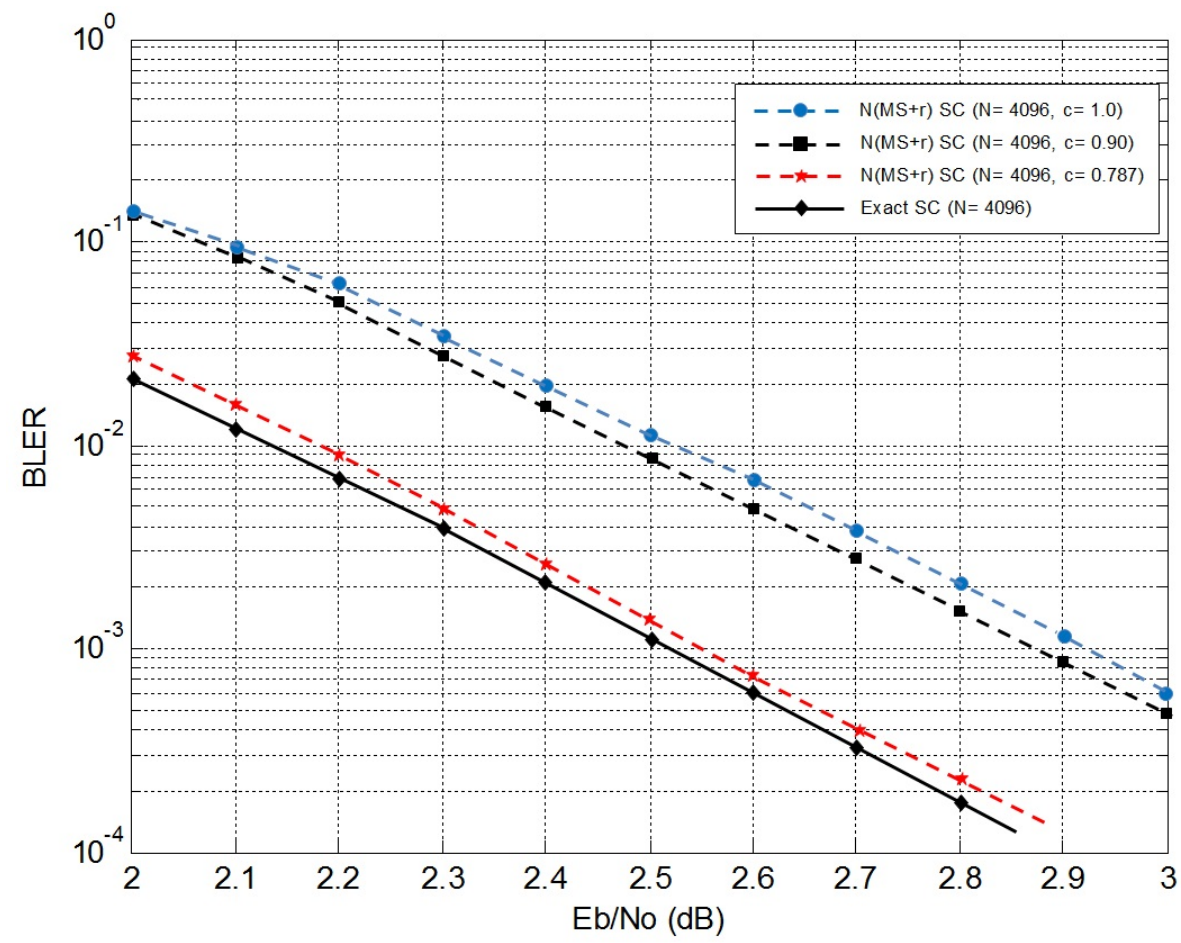

Figure 3.6: Error rates with $N=4096$ and half rate polar code under two different decoding algorithms. 


\section{Chapter 4}

\section{Non-Uniform Quantizers with SC Polar Based Channel-Optimized Decoders}

In real communication systems the achievable rate over a B-DMC with the SC decoder is inferior than the actual channel capacity because of imperfections in the receiver, e.g., limited depth interleaving, imperfect channel estimation, suboptimal detection, etc. $[54,55]$.

Usually, for decoding of information and correction of errors, the receiver should have knowledge of the communication channel parameters. For example, parameters such as noise power, fading gain, timing and synchronization information, etc., should be known at the receiver. Without such information, perfect decoding is usually not possible. Therefore, most communication systems are equipped with channel estimation and timing recovery modules at the receiver side. Error-correction codes are also designed based on the assumption that the receiver has perfect channel knowledge and is perfectly synchronized with the transmitter. Channel estimation and timing techniques, however, are themselves subject to imperfections. They also increase the complexity of the system and can incur a great cost in terms of implementation complexities, required chip area, power dissipation, data overhead, and decoding latency. In modern high-throughput communication systems, the receiver may not be able to handle this extra complexity or overhead. As a solution, a method of scaling the soft information messages before the decoding process can guarantee reduction in the complexity as well as improvement on the achieved throughput of the system [56].

In this chapter we aim to improve the SC decoding algorithm based on approximated calculation of the reliability metrics (LLR values) exchanged during the decoding process. The improvement of the performance is sought via appropriate scaling 
of the LLRs. In details, since LLRs have complex representation then there is a great increasing in the complexity of the LLR calculation metrics making decoders incompetent in manipulating such intricacy in high throughput wireless applications. As a solution, it is found that approximating LLRs with a linear optimized function (i.e. multiplication by a correction factor) of the channel output can improve the performance of the decoding algorithm [57] with low implementation complexity.

In the proposed solution we are looking for optimum scaling factor and then optimum LLR value that maximizes the transmission rate. Although this is a well known method [58], the search for the optimum scaling factor is conventionally based on a brute-force search, which requires extensive simulations. As opposed to this approach, we propose a method that has a low computational complexity, and provides a formal explanation about why a linear scaling factor works in practice. Based on BPSK modulation over uncorrelated Rayleigh fading channel with no channel state information (CSI) at the receiver.

On the other hand, memory is the a hindrance in the implementation of large high speed coding systems. Therefore, if it is possible to find decoders which work with only a few bits per message then this can provide a respect balance achieved between decoder output and hardware realization. So that we also interest in this chapter in designing a quantized polar decoder.

\subsection{Low Complexity LLR Calculation Without Channel State Information}

\subsubsection{System Model}

Suppose $x \in\{-1,+1\}$ is a symbol transmitted over an uncorrelated Rayleigh fading channel using binary phase shift keying (BPSK), and let $y$ be the received sample such that

$$
y=\alpha e^{j \theta} x+n,
$$

where $\alpha, \alpha \geq 0$, is the channel gain, $\theta$ is the channel phase rotation, and $n$ is random noise with zero mean, a variance of $\sigma^{2}$, and has a Gaussian distribution. $\theta$ is uniformly distributed over $[0,2 \pi)$, and while generally unknown at the receiver, is readily estimated by a phase tracking loop. Addressing imperfections in this estimation process 
are beyond the scope of this thesis, and instead we consider the channel model:

$$
y=\alpha x+n
$$

$\alpha$ has probability density function (PDF) of $f(\alpha)=2 \alpha e^{-\alpha^{2}}$ and $\mathrm{E}\left[\alpha^{2}\right]=1$. When $\alpha=1$ then the channel is AWGN (no fading). Depending on knowledge of $\alpha$ at the receiver, we consider two cases:

1) Ideal Channel State Information (Ideal CSI): The Rayleigh fading gain $\alpha$ is known at the receiver with PDF as

$$
f_{y \mid \alpha, x}(y \mid \alpha, x)=\frac{1}{\sqrt{2 \pi \sigma^{2}}} e^{\frac{-(y-\alpha x)^{2}}{2 \sigma^{2}}} .
$$

Hence the LLR, $\lambda$, can be determined in a similar calculation as in (3.29) and gives:

$$
\lambda(y)=\mathrm{LLR}=\ln \frac{f_{y \mid \alpha, x}(y \mid \alpha, x=+1)}{f_{y \mid \alpha, x}(y \mid \alpha, x=-1)}=\frac{2 \alpha}{\sigma^{2}} y,
$$

which has a linear form with $y$.

2) No Channel State Information (No CSI): In this case the CSI is not known at the receiver. The likelihood function of $y$ given $x$, marginalized over $\alpha$, is [59]:

$$
\begin{aligned}
f(y \mid x) & =\int_{0}^{\infty} f_{y \mid \alpha, x}(y \mid \alpha, x) f_{\alpha}(\alpha) \mathrm{d} \alpha \\
& =\int_{0}^{\infty} \frac{1}{\sqrt{2 \pi \sigma^{2}}} e^{\frac{-(y-\alpha x)^{2}}{2 \sigma^{2}}} 2 \alpha e^{-\alpha^{2}} \mathrm{~d} \alpha \\
& =\frac{2 \sigma^{2}}{1+2 \sigma^{2}} \frac{1}{\sqrt{2 \pi \sigma^{2}}} e^{\frac{-(y-\alpha x)^{2}}{2 \sigma^{2}}} \Phi\left(\frac{y x}{\sqrt{2 \sigma^{2}\left(1+2 \sigma^{2}\right)}}\right),
\end{aligned}
$$

when $x \in\{-1,+1\}$, where

$$
\Phi(z)=1+\sqrt{\pi} z e^{z^{2}} \operatorname{erfc}(-z)
$$


The LLR can then be expressed as

$$
\begin{aligned}
\lambda(y) & =\ln \frac{f_{y \mid x}(y \mid x=+1)}{f_{y \mid x}(y \mid x=-1)} \\
& =\ln \frac{\Phi\left(\frac{y}{\sqrt{2 \sigma^{2}\left(1+2 \sigma^{2}\right)}}\right)}{\Phi\left(\frac{-y}{\sqrt{2 \sigma^{2}\left(1+2 \sigma^{2}\right)}}\right)} .
\end{aligned}
$$

Because the expression for the LLR is difficult to evaluate, a linear approximation is often used.

\subsubsection{Linear LLR Approximation}

To reduce the complexity of the LLR calculation when there is no CSI at the receiver, a simple linear approximation has been proposed [59,60]:

$$
\hat{\lambda}=\frac{2 \hat{\alpha}}{\sigma^{2}} y .
$$

This form of approximation is appealing because of its similarity with (4.4), the LLR expression when perfect CSI is available. The scaling factor $\hat{\alpha}$ can be adjusted to control the accuracy of the approximation.

Several different methods have been proposed to select optimal values of $\hat{\alpha}$. Lechner in [61] uses the weighted least-square method such that $\hat{\alpha}=\arg \min _{\alpha>0} \mathrm{E}\left[|\hat{\lambda}-\alpha \lambda|^{2}\right]$. Jilie in [59] used a simple solution of $\hat{\alpha}=\mathrm{E}[\alpha]=\frac{\sqrt{\pi}}{2}$ while in [62] the mutual information is maximized for the optimum factor as $\frac{\mathrm{d}}{\mathrm{d} \alpha} \int_{0}^{\infty} f_{\lambda}(\lambda \mid 0) \log _{2}\left(1+e^{-\lambda}\right) \mathrm{d} \lambda=0$.

\subsubsection{Achievable Rates under Unknown CSI}

An expression for the channel capacity in the absence of CSI at the receiver is presented in this subsection. When the transmitted symbols are constrained to the equiprobably BPSK symbols, $x \in\{-1,+1\}$, the constellation-constrained channel capacity is the average mutual information between $x$ and $y$ :

$$
\mathrm{C}=\mathrm{I}(y ; x)=H(y)-H(y \mid x),
$$


where $H(y)$ and $H(y \mid x)$ are the differential entropy of $y$ and the conditional differential entropy of $y$ given $x$, respectively.

We note that the likelihood function of $y$ given $x$ is symmetric in the sense that

$$
f_{y \mid x}(y \mid x=+1)=f_{y \mid x}(-y \mid x=-1) .
$$

Because of the symmetry property $H(y \mid x=+1)=H(y \mid x=-1)$, so [64]

$$
\begin{aligned}
\mathrm{C}= & H(y)-H(y \mid x=+1) \\
= & -\int_{-\infty}^{\infty} f_{y}(y) \log _{2} f_{y}(y) \mathrm{d} y+\int_{-\infty}^{\infty} f_{y \mid x}(y \mid x=+1) \log _{2} f_{y \mid x}(y \mid x=+1) \mathrm{d} y \\
= & -\int_{-\infty}^{\infty} f_{y \mid x}(y \mid x=+1) \log _{2} f_{y}(y) \mathrm{d} y+\int_{-\infty}^{\infty} f_{y \mid x}(y \mid x=+1) \log _{2} f_{y \mid x}(y \mid x=+1) \mathrm{d} y \\
= & \int_{-\infty}^{\infty} f_{y}(y \mid x=+1) \log _{2} \frac{2 f_{y \mid x}(y \mid x=+1)}{f_{y \mid x}(y \mid x=+1)+f_{y \mid x}(y \mid x=-1)} \mathrm{d} y \\
= & \int_{-\infty}^{\infty} f_{y \mid x}(y \mid x=+1) \log _{2} 2 \mathrm{~d} y \\
& -\int_{-\infty}^{\infty} f_{y \mid x}(y \mid x=+1) \log _{2} \frac{f_{y \mid x}(y \mid x=+1)+f_{y \mid x}(y \mid x=-1)}{f_{y \mid x}(y \mid x=+1)} \mathrm{d} y \\
= & 1-\int_{-\infty}^{\infty} f_{y \mid x}(y \mid x=+1) \log _{2}\left(1+\frac{f_{y \mid x}(y \mid x=-1)}{f_{y \mid x}(y \mid x=+1)} \mathrm{d} y\right. \\
= & 1-\int_{-\infty}^{\infty} f_{y \mid x}(y \mid x=+1) \log _{2}\left(1+e^{-\lambda(y)}\right) \mathrm{d} y,
\end{aligned}
$$

where $f_{y \mid x}(y \mid x=+1)$ is given by (4.5) and $\lambda(y)$ is given by (4.7).

\subsubsection{Optimization of the Scaling Factor}

To find the optimal scaling factor, we apply the following single variable optimization problem [63]

$$
\hat{\alpha}_{\mathrm{opt}}=\arg \max _{\hat{\alpha}>0} \widehat{\mathrm{C}}(\hat{\alpha})
$$

where

$$
\widehat{\mathrm{C}}(\hat{\alpha})=1-\int_{-\infty}^{\infty} f_{y \mid x}(y \mid x=+1) \log _{2}\left(1+e^{-\hat{\lambda}_{\hat{\alpha}}(y)}\right) \mathrm{d} y
$$


is similar to the expression for $\mathrm{C}$ given by (4.11), except that the true LLR, $\lambda(y)$, has been replaced by its linear approximation, $\hat{\lambda}_{\hat{\alpha}}(y)$, given by $(4.8)$.

Since $\mathrm{C}$ represents the true channel capacity then $\widehat{\mathrm{C}} \leqslant \mathrm{C}$ and accordingly under that unique $\hat{\alpha}_{\text {opt }}, \widehat{\mathrm{C}}$ approaches $\mathrm{C}$.

Lemma 5. The approximated channel capacity $(\widehat{\mathrm{C}})$ at the optimum LLR (or at $\left.\hat{\alpha}_{\text {opt }}\right)$ is equal to the true channel capacity achieved by true LLR. This means that $\hat{\alpha}_{\text {opt }}$ is unique and maximizes $\widehat{\mathrm{C}}$. Thus $\widehat{\mathrm{C}}$ acts as a measure of $\hat{\lambda}$ reliability.

Proof. The second derivative of $\widehat{\mathrm{C}}$ can help in proving this claim. We have

$$
\begin{aligned}
\widehat{\mathrm{C}}(\hat{\alpha})=1-\int_{-\infty}^{\infty} f_{y \mid x}(y \mid x=+1) \log _{2}\left(1+e^{-\hat{\lambda}_{\hat{\alpha}}(y)}\right) \mathrm{d} y \\
=1-\int_{0}^{\infty} f_{y \mid x}(y \mid x=+1) \log _{2}\left(1+e^{-\hat{\lambda}_{\hat{\alpha}}(y)}\right) \mathrm{d} y \\
\quad-\int_{-\infty}^{0} f_{y \mid x}(y \mid x=+1) \log _{2}\left(1+e^{-\hat{\lambda}_{\hat{\alpha}}(y)}\right) \mathrm{d} y \\
=1-\int_{0}^{\infty} f_{y \mid x}(y \mid x=+1) \log _{2}\left(1+e^{-\hat{\lambda}_{\hat{\alpha}}(y)}\right) \mathrm{d} y \\
\quad-\int_{-\infty}^{0} f_{y \mid x}(y \mid x=-1) \log _{2}\left(1+e^{\hat{\lambda}_{\hat{\alpha}}(y)}\right) \mathrm{d} y .
\end{aligned}
$$

Then

$\frac{\mathrm{d} \widehat{\mathrm{C}}(\hat{\alpha})}{\mathrm{d} y}=-f_{y \mid x}(y \mid x=+1) \log _{2}\left(1+e^{-\hat{\lambda}_{\hat{\alpha}}(y)}\right)-f_{y \mid x}(y \mid x=-1) \log _{2}\left(1+e^{\hat{\lambda}_{\hat{\alpha}}(y)}\right)$,

and

$$
\frac{\mathrm{d}^{2} \widehat{\mathrm{C}}(\hat{\alpha})}{\mathrm{d} y \mathrm{~d} \hat{\lambda}_{\hat{\alpha}}(y)}=\frac{-1}{\ln 2}\left[-f_{y \mid x}(y \mid x=+1) \frac{e^{-\hat{\lambda}_{\hat{\alpha}}(y)}}{1+e^{-\hat{\lambda}_{\hat{\alpha}}(y)}}+f_{y \mid x}(y \mid x=-1) \frac{e^{\hat{\lambda}_{\hat{\alpha}}(y)}}{1+e^{\hat{\lambda}_{\hat{\alpha}}(y)}}\right] .
$$

The last result shows that the function is concave $(<0)$ and means that $\hat{\alpha}$ is optimum 
only when $\widehat{\mathrm{C}}$ is maximum (Fig. 4.1).

Put $\frac{\mathrm{d}^{2} \widehat{\mathrm{C}}(\hat{\alpha})}{\mathrm{d} y \mathrm{~d} \hat{\lambda}_{\hat{\alpha}}(y)}=0 \quad \Rightarrow$

$f_{y \mid x}(y \mid x=+1) e^{-\hat{\lambda}_{\hat{\alpha}}(y)}\left(1+e^{\hat{\lambda}_{\hat{\alpha}}(y)}\right)=f_{y \mid x}(y \mid x=-1) e^{\hat{\lambda}_{\hat{\alpha}}(y)}\left(1+e^{-\hat{\lambda}_{\hat{\alpha}}(y)}\right) \quad \Rightarrow$

$\frac{f_{y \mid x}(y \mid x=+1)}{f_{y \mid x}(y \mid x=-1)}=\frac{e^{\hat{\lambda}_{\hat{\alpha}}(y)}\left(1+e^{-\hat{\lambda}_{\hat{\alpha}}(y)}\right)}{e^{-\hat{\lambda}_{\hat{\alpha}}(y)}\left(1+e^{\hat{\lambda}_{\hat{\alpha}}(y)}\right)}=\frac{e^{\hat{\lambda}_{\hat{\alpha}}(y) / 2}}{e^{-\hat{\lambda}_{\hat{\alpha}}(y) / 2}}=e^{\hat{\lambda}_{\hat{\alpha}}(y)}$.

Or $\hat{\lambda}_{\hat{\alpha}}(y)=\ln \frac{f_{y \mid x}(y \mid x=+1)}{f_{y \mid x}(y \mid x=-1)}=$ True LLR.

A recursive method to calculate $\hat{\alpha}_{\text {opt }}$ is given below.

Initialization: set $\hat{\alpha}=0.3$ and $s=1.2$.

1. Calculate $\widehat{\mathrm{C}}(\hat{\alpha})$ and $\widehat{\mathrm{C}}(s \hat{\alpha})$ according to $\widehat{\mathrm{C}}=1-\int_{0}^{\infty} f_{\hat{\lambda}}(\hat{\lambda}) \log _{2}\left(1+e^{-\hat{\lambda}}\right) \mathrm{d} \lambda$ where $\hat{\lambda}=\frac{2 \hat{\alpha}}{\sigma^{2}} y$.

2. If $\widehat{\mathrm{C}}(\hat{\alpha})>\widehat{\mathrm{C}}(s \hat{\alpha})$, set $s=\frac{1}{s}$. Otherwise, let $\widehat{\mathrm{C}}(\hat{\alpha})=\widehat{\mathrm{C}}(s \hat{\alpha})$, set $\hat{\alpha}=s \hat{\alpha}$.

3. Calculate $\widehat{\mathrm{C}}(s \hat{\alpha})$. If $\widehat{\mathrm{C}}(\hat{\alpha})>\widehat{\mathrm{C}}(s \hat{\alpha})$, set $\hat{\alpha}_{\text {opt }}=\frac{\hat{\alpha}+s \hat{\alpha}}{2}$. Stop.

4. Otherwise, let $\widehat{\mathrm{C}}(\hat{\alpha})=\widehat{\mathrm{C}}(s \hat{\alpha})$, set $\hat{\alpha}=s \hat{\alpha}$. Go back to step (3).

Fig. 4.1 illustrates different $\widehat{\mathrm{C}}$ curves with respect to $\hat{\alpha}$ for different values of $\sigma$. For $\sigma=0.5, \hat{\alpha}_{\mathrm{opt}}=0.461$, for $\sigma=0.6, \hat{\alpha}_{\mathrm{opt}}=0.597$ and for $\sigma=0.7, \hat{\alpha}_{\mathrm{opt}}=0.638$. As noticed, each one of the optimum (which maximizes the capacity) values is unique. It can be seen from Fig. 4.2 how close the performance of the proposed approximation is to the true channel capacity over the uncorrelated Rayleigh fading channel. Fig. 4.3 shows an LLR comparison between the true LLR obtained from (4.7) and other linear approximated versions, LLR based on $\hat{\alpha}=\mathrm{E}[\alpha]$ and LLR under $\hat{\alpha}=\hat{\alpha}_{\text {opt }}$. It is noticed that both the approximated curves diverge at high values of $y$.

Even though neither linear approximation for the LLR appears accurate, the perfor- 


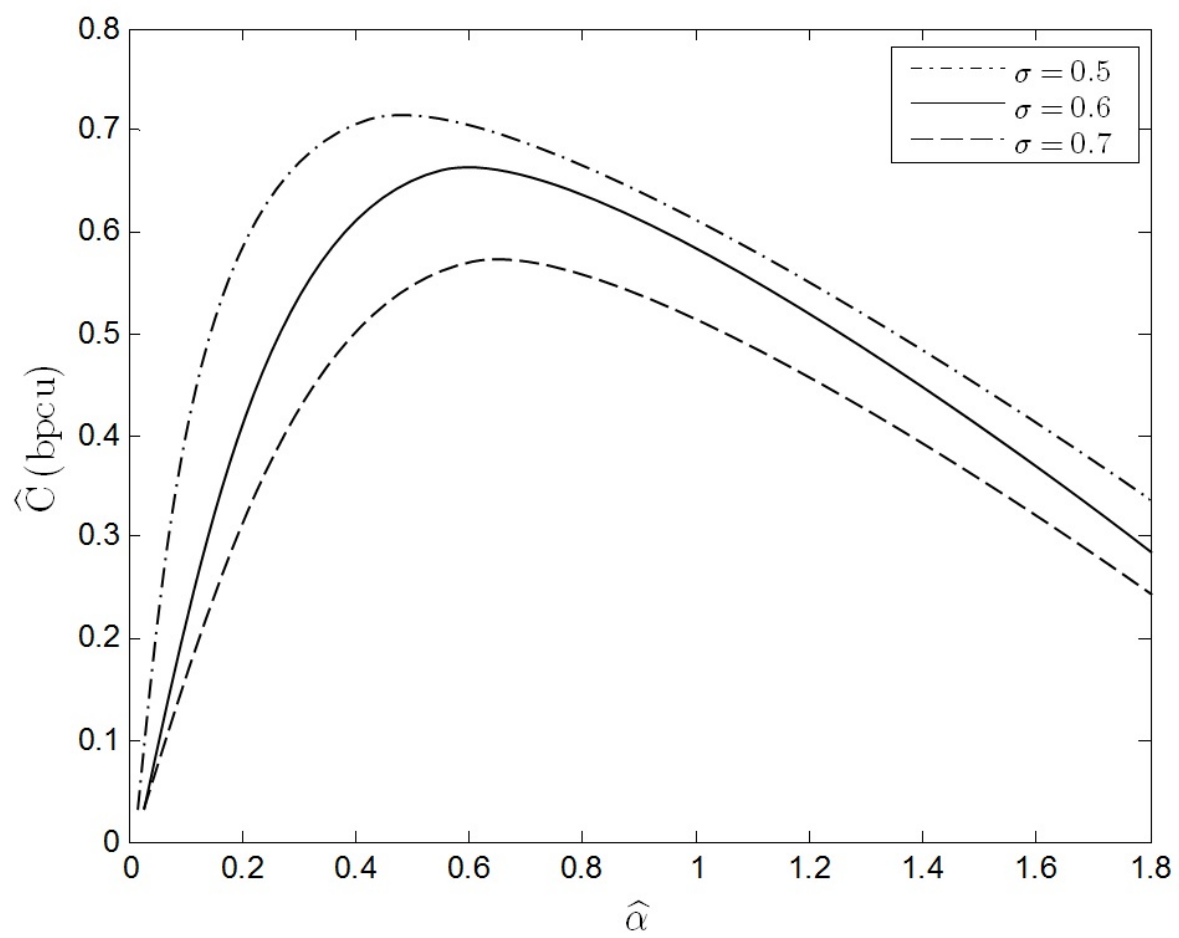

Figure 4.1: The achievable information rate under multiple values of $\sigma$.

mance of an SC decoded polar code is not adversely impacted when the optimized linear approximation proposed here is used, as shown in Fig. 4.4. The best achieved $\mathrm{SC}$ polar decoding threshold is $3.74 \mathrm{~dB}$ at $\hat{\alpha}_{\mathrm{opt}}=0.627$.

As a result and according to all above figures, it is observed that the approach we presented gives results very close to the true LLR calculation (4.7) performance over the uncorrelated Rayleigh fading channel with unknown CSI; with the significant advantage of very low LLR computational complexity.

\subsection{Non-Uniform Quantization for SC Polar codes}

\subsubsection{Effects of Quantization on Channel Capacity}

In this section, we focus on understanding how quantization affects the performance of the SC polar soft-output decoder. First, we begin by demonstrating the effects of the number of quantization bits on the channel capacity.

We consider a binary communication system where a single bit, $\tilde{x} \in\{0,1\}$, is mapped to a BPSK symbol $x \in\{-1,+1\}$ as $x=1-2 \tilde{x}$ which is transmitted over a 


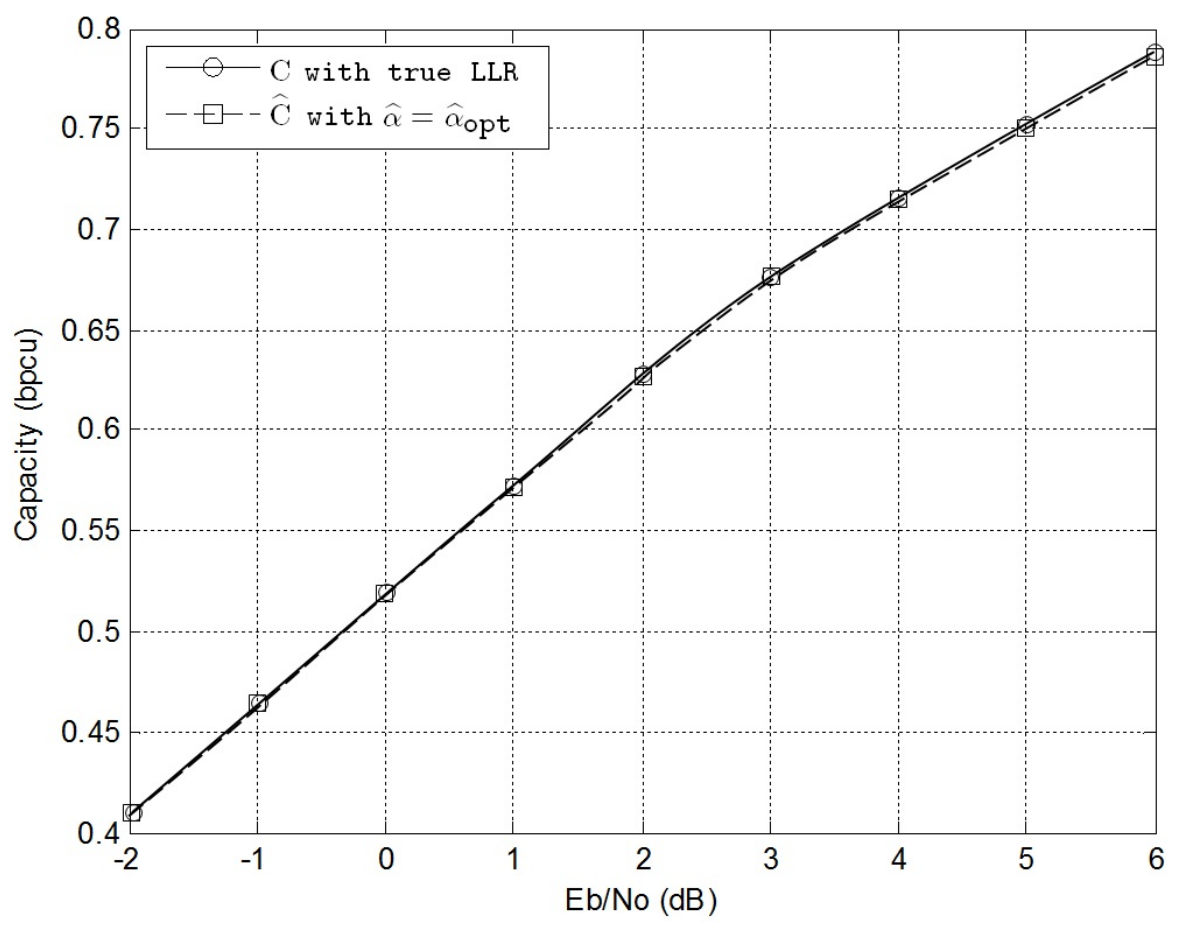

Figure 4.2: $\mathrm{C}$ under true LLR compared with $\widehat{\mathrm{C}}$ under $\hat{\alpha}_{\text {opt }}=0.627$.

Rayleigh fading channel, either with or without CSI at the receiver, yielding received sample $y \in \mathbb{R}$. The received sample is quantized to one of $L$ distinct levels, giving $\tilde{y}=\mathbf{Q}(y) \in\{0,1, \ldots, L-1\}$, according to the quantization rule $\mathbf{Q}(y)=j$ if $y \in N_{j}$ for $j \in\{0,1, \ldots, L-1\}$, where the $j$ th quantization interval is $N_{j}=\left(n_{j}, n_{j+1}\right]$ where $n_{j}$ is the boundary between $N_{j-1}$ and $N_{j}$ with $n_{0}=-\infty$ and $n_{L}=\infty$.

As an example, consider the symmetric uniform quantization rule with $L=5$ levels shown in Fig. 4.5. This is uniform in the sense that all intervals have the same width, $\Delta$ (i.e. $n_{j+1}-n_{j}=\Delta \quad \forall j \in[1,2, \ldots, L-2]$ ), and symmetric in the sense that $n_{j}=-n_{L-j} \quad \forall j$.

Associated with the quantization scheme are the channel transition probabilities [65].

$$
\begin{aligned}
f_{i j} & \equiv \operatorname{Pr}\{\tilde{y}=j \mid \tilde{x}=i\} \\
& =\operatorname{Pr}\left\{y \in N_{j} \mid \tilde{x}=i\right\} \\
& =\int_{N_{j}} f(y \mid x=1-2 i) \mathrm{d} y,
\end{aligned}
$$




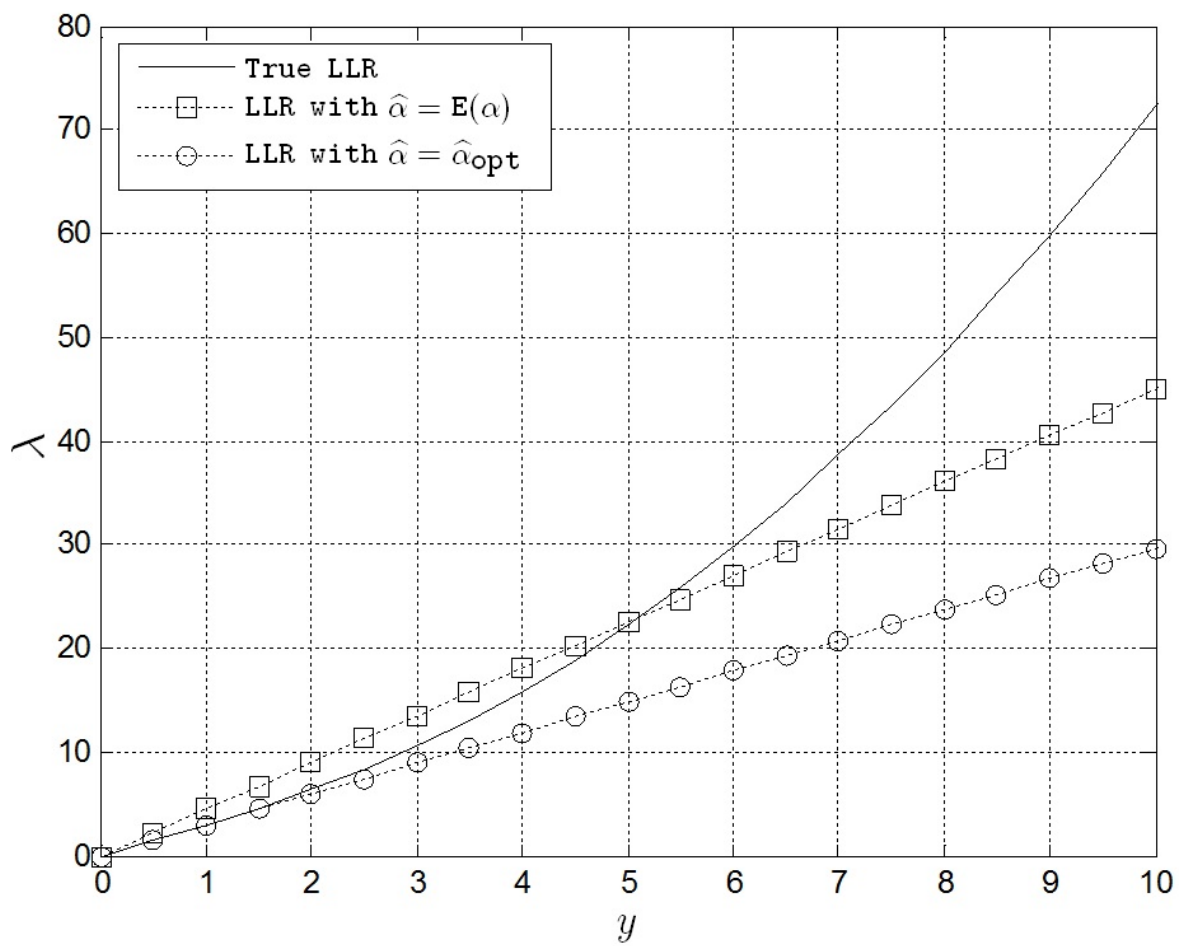

Figure 4.3: True LLR compared to two approximated LLRs under $\sigma=0.65$, LLR with $\hat{\alpha}=\mathrm{E}[\alpha]=\frac{\sqrt{\pi}}{2}$ and LLR with $\hat{\alpha}_{\text {opt }}=0.627$.

where $f(y \mid x)$ is the likelihood function of the underlying unquantized channel. Because of the symmetry properties of the unquantized channel and the quantization rule, we have $f_{0 j}=f_{1(L-1-j)}$, and so the capacity of the quantized channel can be expressed as $[66,67]$

$$
\begin{aligned}
\mathrm{C}_{\mathrm{q}} & =H(y)-H(\tilde{y} \mid \tilde{x}) \\
& =1-\sum_{j=0}^{L-1} \hat{f}_{0 j} \log _{2}\left(1+\frac{\hat{f}_{1 j}}{\hat{f}_{0 j}}\right),
\end{aligned}
$$

where $\mathrm{q}$ is number of bits required to identify the quantized value.

To investigate the effects of quantization on capacity and polar code performance, we consider uniform quantization scheme with $L=2^{\mathrm{q}}$ levels and with a separation between levels of $\Delta$. The most relevant results are presented in Fig. 4.6. The results show that 6 -bit uniform quantization with $\Delta=0.025$ offers nearly the same capacity as unquantized demodulation, exhibiting unnoticeable SNR loss. The results also 


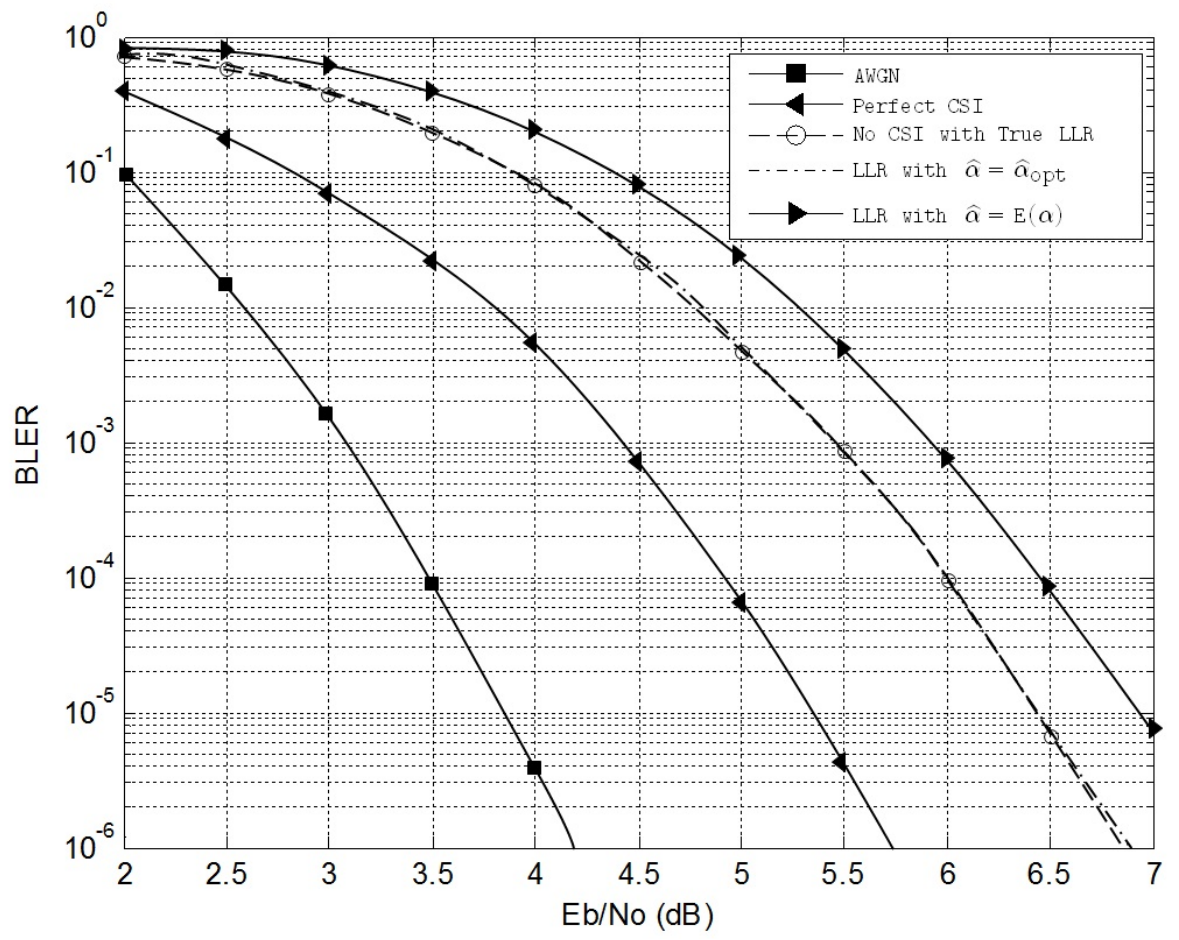

Figure 4.4: BLER under different LLR calculation for SC polar code length of 1024 and rate 0.5 .

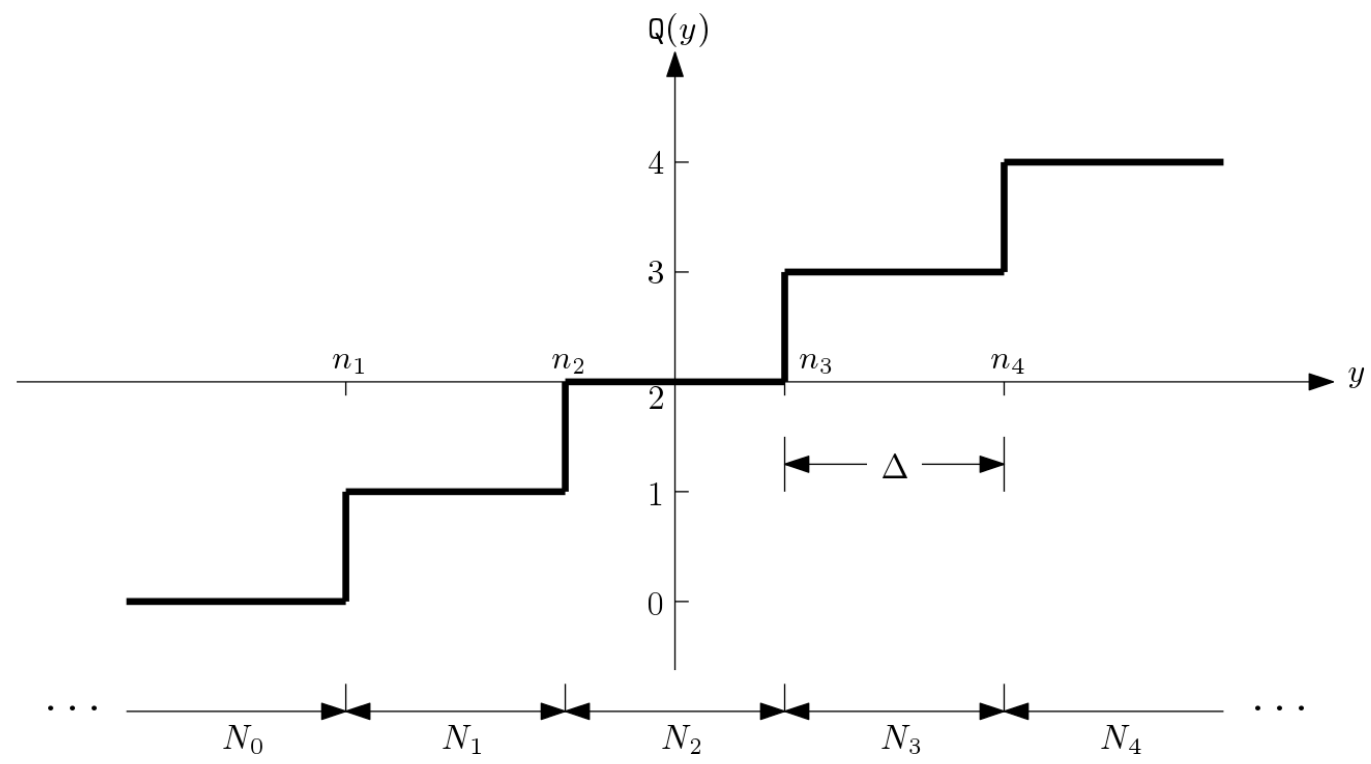

Figure 4.5: The quantization scheme.

show that $\Delta=0.05$ is worse than $\Delta=0.025$ at high SNRs. Fig. 4.7 shows the block error rate (BLER) of a SC decoded polar code with and without quantization. The 
performance with $\mathrm{q}=5$ is poor while when $\mathrm{q}=6$ the gap with the unquantized one is around $0.1 \mathrm{~dB}$.

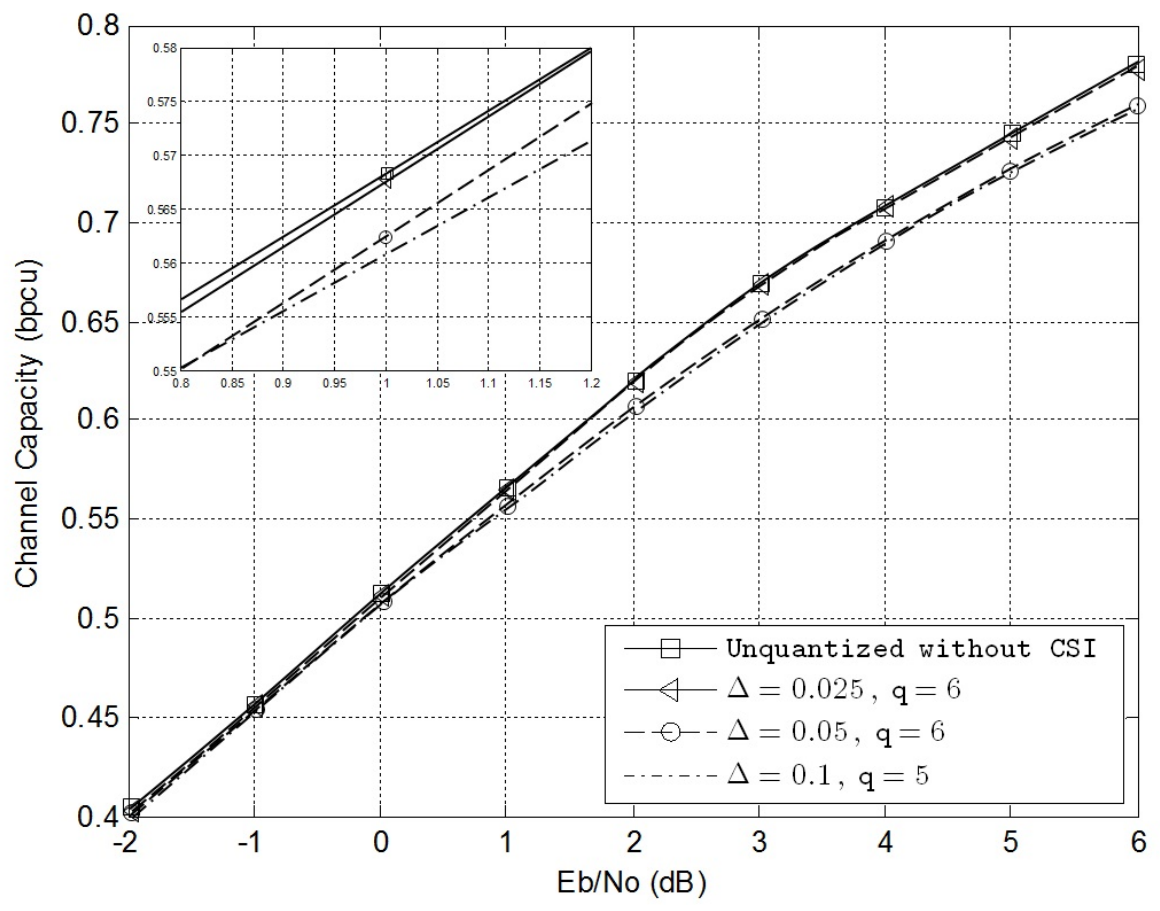

Figure 4.6: Capacity for (un)quantized channel without CSI measurements based on LLR optimized linear approximation with $\hat{\alpha}_{\text {opt }}=0.627$.

\subsubsection{Non-Uniform Quantization Scheme}

Although the performance differences shown in Fig. 4.7 between the unquantized and the 6-bit quantized schemes are almost undetectable, it is always desirable to use fewer quantization bits if possible, in order to reduce the decoder complexity.

Let us recall the function $f(x)$ explained in Subsection 3.3.1, $f(x)=\ln \frac{e^{x}+1}{e^{x}-1}$, which is shown in Fig. 4.8. We can see that $f(x)$ decreases logarithmically as $x$ increases. In detail, there are two slope regions of $f(x)$ as shown, the floor and water-fall regions. The sharp slope in water-fall region requires more output bits while the converse is true in the floor region due the slender slope (less required bits). Thus, this suggests the use of non-uniform quantization to better capture the intricacy of $f(x)$ in a look-up table (LUT) realization $[68,69]$. 


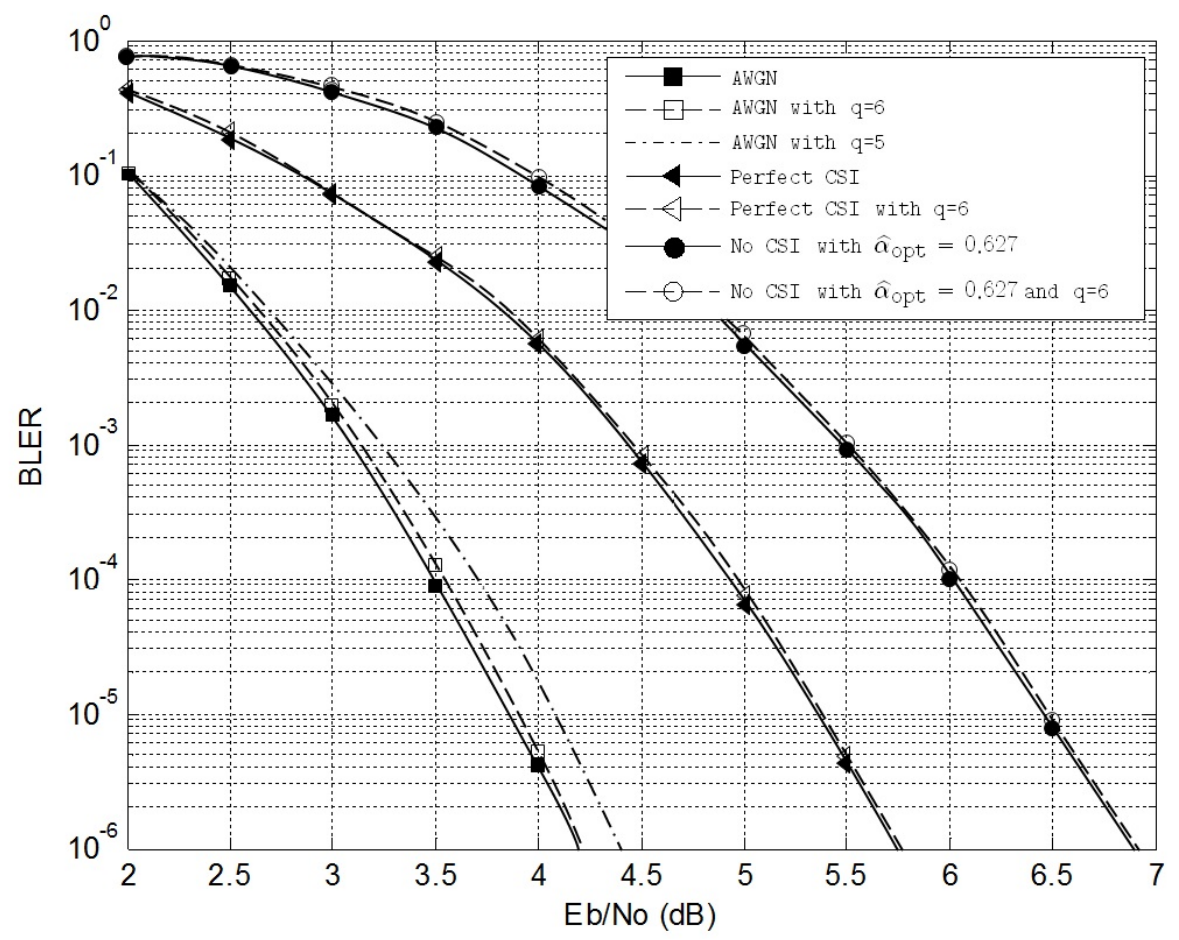

Figure 4.7: Simulated BLER for the length 1024 and rate 0.5 SC polar codes with different decoding scenarios.

Let $(q, f)$ represents a fixed-point number with $f$ fractional bits $(f=3$ beside 1 sign bit per data as well there are 2 integer bits so that there are $q=6$ bits in total). However, with the following quantizing rule the LUT inputs are lowered by half (Table 4.1).

With $\mathrm{q}=6$ and $\mathrm{f}=3$ we get

$$
\begin{array}{ll}
(6,3) \text { and } \Delta=0.03125 & \text { for } 0<x<1 \\
(5,2) \text { and } \Delta=0.0625 & \text { for } 1<x<2 \\
(4,1) \text { and } \Delta=0.125 & \text { for } 2<x<4 .
\end{array}
$$


CHAPTER 4. OPTIMIZED SC DECODER BASED APPROXIMATED LLRS 64

Table 4.1: Uniform and non-uniform quantization methods with $q=6$ and $f=3$

\begin{tabular}{|c|c|c|c|}
\hline \multirow[b]{2}{*}{ Input $=2^{q-1}$} & \multicolumn{3}{|c|}{ Output } \\
\hline & Decimal & Uniform Quantization & Method \\
\hline 00000 & 4.000 & 10000 & 10000 \\
\hline 00001 & 3.250 & 01101 & 01101 \\
\hline 00010 & 2.681 & 01010 & 01010 \\
\hline 00011 & 2.343 & 01001 & 01001 \\
\hline 00100 & 1.571 & 00110 & 00110 \\
\hline 00101 & 1.255 & 00101 & 00101 \\
\hline 00110 & 1.000 & 00100 & 00100 \\
\hline 00111 & 0.810 & 00011 & 00011 \\
\hline 01000 & 0.748 & 00010 & \multirow{2}{*}{00010} \\
\hline 01001 & 0.650 & 00010 & \\
\hline 01010 & 0.600 & 00010 & \multirow{2}{*}{00010} \\
\hline 01011 & 0.533 & 00010 & \\
\hline 01100 & 0.458 & 00001 & \multirow{2}{*}{00001} \\
\hline 01101 & 0.443 & 00001 & \\
\hline 01110 & 0.370 & 00001 & \multirow{2}{*}{00001} \\
\hline 01111 & 0.261 & 00001 & \\
\hline 10000 & 0.200 & 00000 & \multirow{4}{*}{00000} \\
\hline 10001 & 0.171 & 00000 & \\
\hline 10010 & 0.112 & 00000 & \\
\hline 10011 & 0.108 & 00000 & \\
\hline 10100 & 0.108 & 00000 & \multirow{4}{*}{00000} \\
\hline 10101 & 0.100 & 00000 & \\
\hline 10110 & 0.100 & 00000 & \\
\hline 10111 & 0.100 & 00000 & \\
\hline 11000 & 0.096 & 00000 & \multirow{4}{*}{00000} \\
\hline 11001 & 0.050 & 00000 & \\
\hline 11010 & 0.020 & 00000 & \\
\hline 11011 & 0.020 & 00000 & \\
\hline 11100 & 0.000 & $00000)$ & \multirow{4}{*}{00000} \\
\hline 11101 & 0.000 & 00000 & \\
\hline 11110 & 0.000 & 00000 & \\
\hline 11111 & 0.000 & 00000 & \\
\hline
\end{tabular}




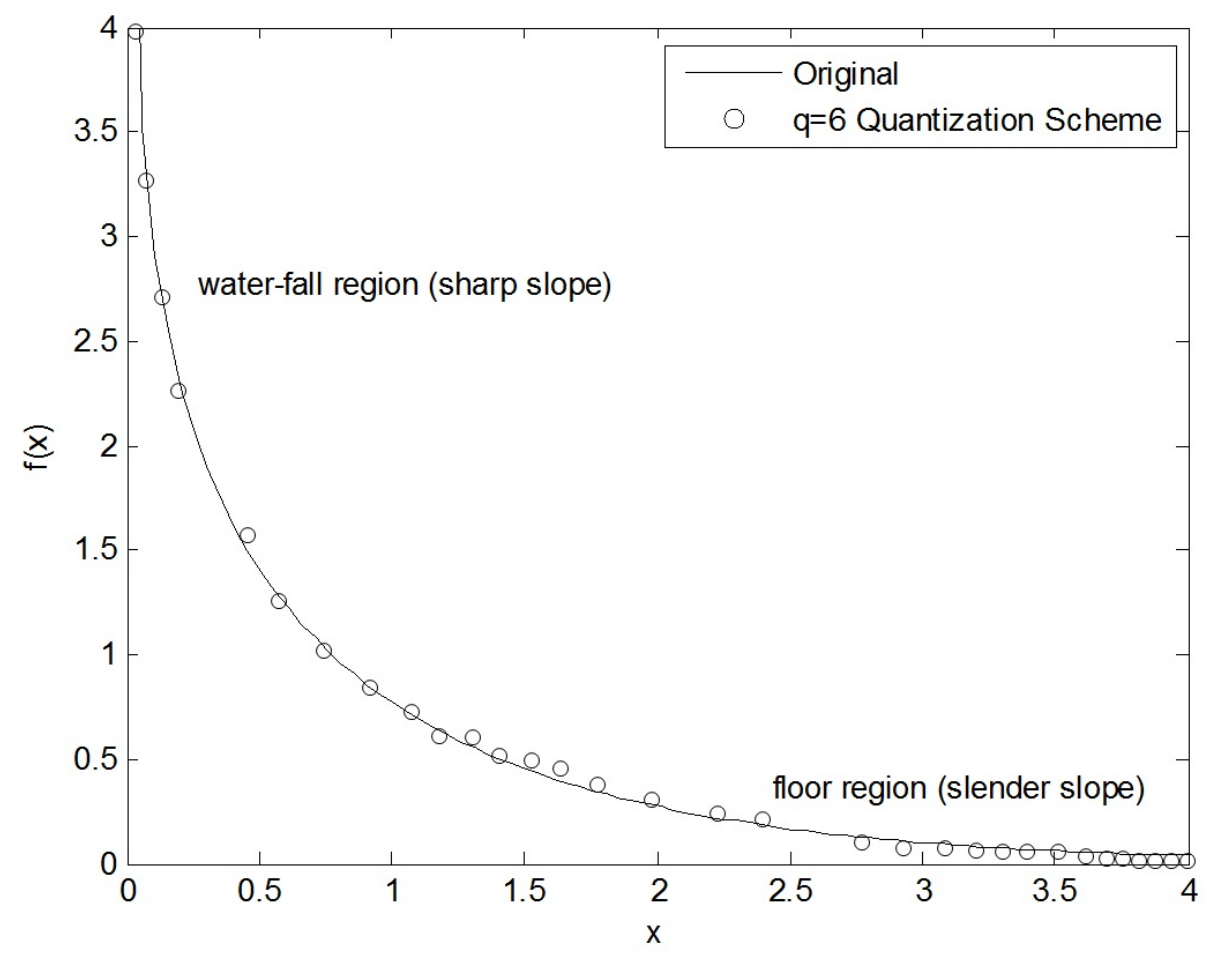

Figure 4.8: The nonlinear function $f(x)=\ln \frac{e^{x}+1}{e^{x}-1}, x>0$.

\subsection{Numerical Results}

We simulate the transmission of polar codes under BPSK modulation and over uncorrelated Rayleigh fading channel with no CSI at the receiver. We also generalize the test by assuming an AWGN channel and with perfect CSI. In Fig. 4.9, we depict the performance for the unquantized optimized linear LLR approximation algorithm when $\hat{\alpha}_{\text {opt }}=0.627$ and observe that the non-uniform quantization (NUQ) method achieves nearly the same performance as the uniform quantized one with $\mathrm{q}=6$. Thus, the NUQ SC polar decoder can be realized with low complication and with no considerable loss.

\subsection{Summary}

In this chapter, we presented a capacity maximization scheme for quantizer design of polar codes over Rayleigh fading channel without CSI at the receiver. Simulations show that a non-uniform quantizer is able to reach the floating point performance. From the numerical results the suggested method for polar decoder showed $50 \%$ 


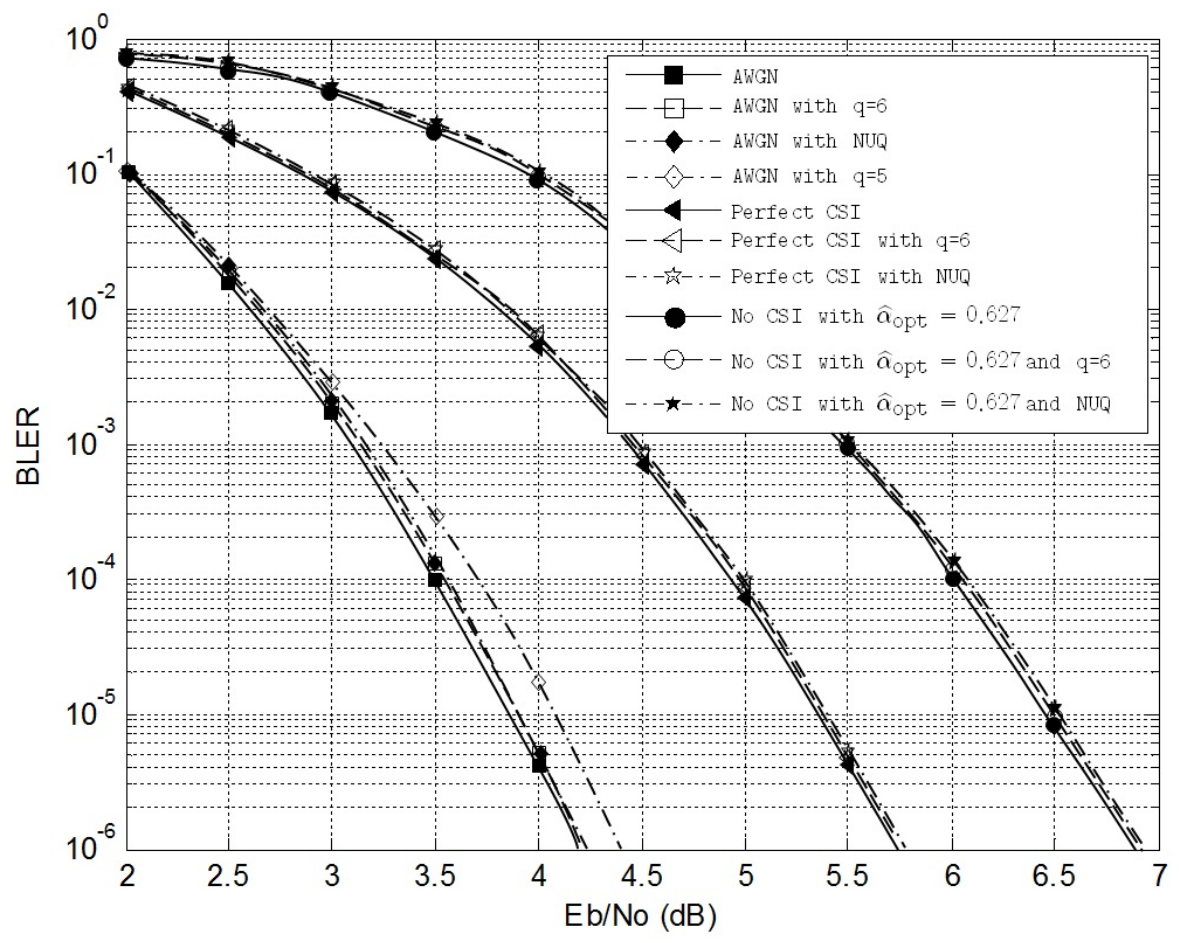

Figure 4.9: Simulated BLER for the length 1024 and rate 0.5 SC polar codes with different decoding scenarios. NUQ stands for non-uniform quantization.

lowering in hardware realization with no considerable loss in performance as compared with uniform quantized decoders. 


\section{Chapter 5}

\section{Low Computational Complexity of SC Polar Decoder in MIMO Fading Channel}

A significant amount of work has been reported to analyze the performance of multiple-input multiple-output (MIMO) systems in combination with countermeasures, such as error-correcting codes [70-74]. Among all the error-correcting codes, polar codes can accomplish the bit error rate (BER) performance approaching Shannon limit [75-79]. In this chapter, we discuss a concatenation scheme of polar codes and Alamouti encoder for space-time block codes. The channel capacity $\mathrm{C}_{\mathrm{M} \text {-QAM }}$ is defined as the maximum information rate found when quadrature amplitude constellation of size M (M-QAM) is applied. This information rate has upper limit due $\mathrm{C}$, the channel capacity, whereas the "reduction" amount in achievable rate between them can be referred due to the the logarithm of the constellation size and the channel SNR [80,81]. Accordingly, the channel capacity C which grows logarithmically diverges from the bounded $\mathrm{C}_{\mathrm{M}-\mathrm{QAM}}$ at high values of $\mathrm{SNR}$ while they are close together at low SNRs.

First, we try to amplify the transmitted signal amplitude by scaling the points with a factor $>0$ in order to approach $\mathrm{C}_{\mathrm{M} \text {-QAM }}$ with the channel capacity. Also, we characterize the optimal distribution through using Hermite polynomials theory under an even-moment constraint. Second, we show that the obtained optimal input distribution can be factored into two identical distributions, and then, as a result, apply the same optimization algorithm on the new input distribution. This step can hugely lower the complexity of optimization computations along the algorithm.

The contributions thus lie in the study of the capacity of this MIMO channel 
model, in which we show that 1) $\mathrm{C}_{\mathrm{M}-\mathrm{QAM}}$ is computed with two constraints, finitesize input signal constellation constraint and a maximum power constraint, 2) the capacity-achieving distribution, subject to an even-moment constraint $\mathrm{E}\left[x^{H} x\right] \leq P$ for some $P>0$, is shown to be optimal, Gaussian, and continuous, and 3) the optimal input symbol distribution, numerically indicated by the results of an optimization procedure based Hermite polynomials theory, is factored into Cartesian product of symmetrical distributions.

\subsection{System Model}

Consider a complex representation of a MIMO communication channel, which has $T$ transmitting antennas, satisfying a power constraint $P$, and $R$ receiving antennas given by:

$$
\mathbf{y}=\mathbf{H} \mathbf{x}+\mathbf{n}, \quad \mathrm{E}\left[\mathbf{x}^{\mathrm{H}} \mathbf{x}\right] \leqslant P .
$$

Where $\mathbf{y} \in \mathbb{C}^{R}$ represents the received signal, the column vector $\mathbf{x}=$ $\left(x_{1}, x_{2}, \ldots, x_{\mathrm{M}}\right)^{\mathrm{T}} \in \mathbb{C}^{T}$ is the transmitted signal with the constellation size of $\mathrm{M}$, and $\mathbf{H} \in \mathbb{C}^{T \times R}$ is the channel gain matrix of random coefficients $h_{i j}(i \in[1, \ldots, T], j \in$ $[1, \ldots, R])$ with zero mean and unit variance. The complex noise vector $\mathbf{n} \in \mathbb{C}^{R}$ has independent and identically distributed (i.i.d) zero-mean Gaussian random variables with variance of $\sigma_{n}^{2}$, and $P$ is the transmitted signal power. Additionally, it is assumed that the channel state information (CSI) is known at the receiver side.

Fig. 5.1 shows the signal constellation diagram of the quadrature amplitude modulation (QAM) with constellation size of $\mathrm{M}=16$ ( $\left.\mathcal{X}_{16-\mathrm{QAM}}\right)$. The conditional probability density function $(\mathrm{PDF})$ is given by:

$$
p(y \mid \mathbf{H} x)=\left(\frac{1}{\sqrt{2 \pi \sigma^{2}}}\right)^{R} e^{-\frac{\|y-h x\|^{2}}{2 \sigma_{n}^{2}}},
$$

and the channel capacity of the MIMO channel is defined as

$$
\mathrm{C}=\sup _{p} \mathrm{I}(\mathbf{x} ; \mathbf{y}) \quad \text { bits } / \mathrm{sec} / \mathrm{Hz}
$$




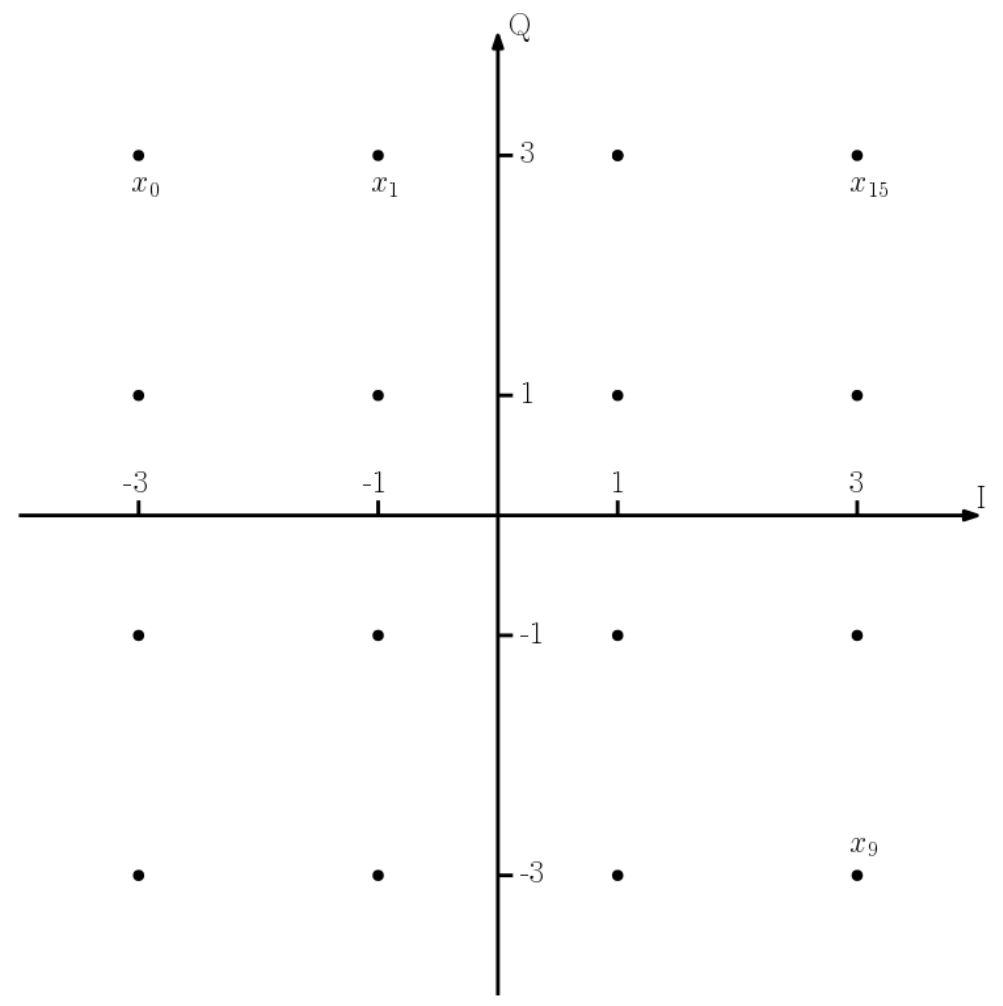

Figure 5.1: Signal constellation of $\mathcal{X}_{16-\mathrm{QAM}}$.

with $\mathrm{I}(\mathbf{x} ; \mathbf{y})$ is the mutual information that can be given as follows:

$$
\mathrm{I}(\mathbf{x} ; \mathbf{y})=\int p(y \mid \mathbf{H} x) \log _{2} \frac{p(y \mid \mathbf{H} x)}{p(y \mid \mathbf{H})} \mathrm{d} h \mathrm{~d} y
$$

and

$$
p(y \mid \mathbf{H})=\sum_{x_{i} \in \mathcal{X}_{16-\mathrm{QAM}}} p\left(x_{i}\right) p(y \mid \mathbf{H} x) .
$$

Now, let us first define the marginal output density of y corresponding to the input distribution $\mathrm{Q}(\mathrm{x})$ as

$$
p(y ; \mathrm{Q}(x))=\int p(y \mid \mathbf{H} x) \mathrm{dQ}(x) .
$$

Then with an input power constraint, the optimization problem can be formulated 
as

$$
\begin{aligned}
\mathrm{C}= & \sup _{\substack{\mathrm{Q} \in \mathcal{Q} \\
\mathrm{E}\left[\mathbf{x}^{\mathrm{H}} \mathbf{x}\right] \leqslant P}} \mathrm{I}(\mathrm{Q}(x)) \\
= & \sup _{\substack{\mathrm{Q} \in \mathcal{Q} \\
\mathrm{E}\left[\mathbf{x}^{\mathrm{H}} \mathbf{x}\right] \leqslant P}} \iint p(y \mid \mathbf{H} x) \log _{2} \frac{p(y \mid \mathbf{H} x)}{p(y ; \mathrm{Q}(x))} \mathrm{d} y \mathrm{dQ}(x)
\end{aligned}
$$

with $\mathcal{Q}$ is the set of all distributions that satisfy the average power constraint [82,83], i.e.,

$$
\mathcal{Q}=\mathrm{Q}: \int_{-\infty}^{\infty} x^{2} \mathrm{dQ}(x) \leqslant P .
$$

As it is not easy to solve the supremum of the last equation, convex optimization theory can be utilized to show that a unique variable $x^{*}$ with its input distribution CDF $\mathrm{Q}\left(x^{*}\right)$ attains the capacity $\mathrm{C}$ [84]. The required conditions are as the following:

$$
\mathrm{C} \geqslant \lambda\left(P-\|\mathbf{x}\|^{2}\right)+\iint p(y \mid \mathbf{H} x) \log _{2} \frac{p(y \mid \mathbf{H} x)}{p(y ; \mathrm{Q}(x))} \mathrm{d} y \mathrm{dQ}(x),
$$

for all $x$, then

$$
\mathrm{C}=\mathrm{I}\left(\mathrm{Q}\left(x^{*}\right)\right)=\lambda^{*}\left(P-\left\|\mathbf{x}^{*}\right\|^{2}\right)+\int p(y \mid \mathbf{H} x) \log _{2} \frac{p(y \mid \mathbf{H} x)}{p\left(y ; \mathrm{Q}\left(x^{*}\right)\right)} \mathrm{d} y,
$$

where $\lambda^{*}=\lambda(P) \geqslant 0$ denotes the optimal Lagrange multiplier.

\subsection{The Problem}

In this section the core results of the work are derived. The purpose here is to characterize the capacity-approaching input structure for the coherent Rayleigh fading channel with Gaussian noise.

Specifically, we will first explain the problem in solving (5.10) above and how can Hermite polynomials help to reach solving the integration. Following that, we will demonstrate that there exists a unique solution of the optimal input distribution and this distribution has Gaussian form. 


\subsubsection{Capacity-Achieving Distribution Characterization}

It is necessary to point out some properties of the mutual information, $\mathrm{I}\left(\mathrm{Q}\left(x^{*}\right)\right)$, where I $\left(\mathrm{Q}\left(x^{*}\right)\right)=\int p(y \mid \mathbf{H} x) \log _{2} \frac{p(y \mid \mathbf{H} x)}{p\left(y ; \mathrm{Q}\left(x^{*}\right)\right)} \mathrm{d} y$.

Lemma 6. For a Rayleigh fading channel giving in (5.1) with input distribution $\mathrm{Q}(\mathrm{x})$, the integration $\int p(y \mid \mathbf{H} x) \log _{2} \frac{p(y \mid \mathbf{H} x)}{p\left(y ; \mathrm{Q}\left(x^{*}\right)\right)} \mathrm{d} y$ satisfies the following properties:

a) $\lim _{x \rightarrow \infty} \int p(y \mid \mathbf{H} x) \log _{2} \frac{p(y \mid \mathbf{H} x)}{p\left(y ; \mathrm{Q}\left(x^{*}\right)\right)} \mathrm{d} y \approx-\log _{2}\left[p\left(y ; \mathrm{Q}\left(x^{*}\right)\right)\right]$.

b) $\int p(y \mid \mathbf{H} x) \log _{2} \frac{p(y \mid \mathbf{H} x)}{p\left(y ; \mathrm{Q}\left(x^{*}\right)\right)} \mathrm{d} y<-\log _{2}\left[p\left(y ; \mathrm{Q}\left(x^{*}\right)\right)\right]$.

Proof. For any finite noise variance $\sigma^{2}$, as $x \longrightarrow \infty$ the conditional PDF $p(y \mid \mathbf{H} x)$ tends to unity. This leads to

$$
\begin{aligned}
& \lim _{x \rightarrow \infty} \int p(y \mid \mathbf{H} x) \log _{2} \frac{p(y \mid \mathbf{H} x)}{p\left(y ; \mathrm{Q}\left(x^{*}\right)\right)} \mathrm{d} y \\
& =\lim _{x \rightarrow \infty} \int p(y \mid \mathbf{H} x) \log _{2} p(y \mid \mathbf{H} x) \mathrm{d} y-\lim _{x \rightarrow \infty} \int p(y \mid \mathbf{H} x) \log _{2} p\left(y ; \mathrm{Q}\left(x^{*}\right)\right) \mathrm{d} y \\
& \approx 0-\log _{2}\left[p\left(y ; \mathrm{Q}\left(x^{*}\right)\right)\right] \\
& =-\log _{2}\left[p\left(y ; \mathrm{Q}\left(x^{*}\right)\right)\right]
\end{aligned}
$$

To prove part $\mathbf{b}$, we notice that the integral $\int p(y \mid \mathbf{H} x) \log _{2} \frac{p(y \mid \mathbf{H} x)}{p\left(y ; \mathrm{Q}\left(x^{*}\right)\right)} \mathrm{d} y$ is always 
positive, $\int p(y \mid \mathbf{H} x) \log _{2} \frac{p(y \mid \mathbf{H} x)}{p\left(y ; \mathrm{Q}\left(x^{*}\right)\right)} \mathrm{d} y \geqslant 0$, then

$$
\begin{aligned}
& \int p(y \mid \mathbf{H} x) \log _{2} \frac{p(y \mid \mathbf{H} x)}{p\left(y ; \mathrm{Q}\left(x^{*}\right)\right)} \mathrm{d} y \\
& =-\int p(y \mid \mathbf{H} x) \log _{2} \frac{p\left(y ; \mathrm{Q}\left(x^{*}\right)\right)}{p(y \mid \mathbf{H} x)} \mathrm{d} y \\
& \geqslant-\log _{2} \int p(y \mid \mathbf{H} x) \frac{p\left(y ; \mathrm{Q}\left(x^{*}\right)\right)}{p(y \mid \mathbf{H} x)} \mathrm{d} y \quad \text { by Jensen's inequality } \\
& =-\log _{2} \int p(y \mid \mathbf{H} x) p\left(y ; \mathrm{Q}\left(x^{*}\right)\right) \mathrm{d} y \\
& =0 . \\
& \Longrightarrow \int p(y \mid \mathbf{H} x) \log _{2} \frac{p(y \mid \mathbf{H} x)}{p\left(y ; \mathrm{Q}\left(x^{*}\right)\right)} \mathrm{d} y \geqslant 0
\end{aligned}
$$

Or $\underbrace{\int p(y \mid \mathbf{H} x) \log _{2} p(y \mid \mathbf{H} x) \mathrm{d} y}_{<0}<-\int p(y \mid \mathbf{H} x) \log _{2} p\left(y ; \mathrm{Q}\left(x^{*}\right)\right) \mathrm{d} y$.

The entropy is bounded

$\Longrightarrow \int p(y \mid \mathbf{H} x) \log _{2} p(y \mid \mathbf{H} x) \mathrm{d} y<-\log _{2}\left[p\left(y ; \mathrm{Q}\left(x^{*}\right)\right)\right]$ by the logarithm of the alphabet size $\left(\left|\mathrm{Q}\left(x^{*}\right)\right|<\right.$ $\infty)[86]$

$\Longrightarrow \int p(y \mid \mathbf{H} x) \log _{2} \frac{p(y \mid \mathbf{H} x)}{p\left(y ; \mathrm{Q}\left(x^{*}\right)\right)} \mathrm{d} y<-\log _{2}\left[p\left(y ; \mathrm{Q}\left(x^{*}\right)\right)\right]$.

Now

$$
\begin{aligned}
\mathrm{C}= & \lambda^{*}\left(P-\left\|\mathbf{x}^{*}\right\|^{2}\right)+\left(\frac{1}{\sqrt{2 \pi \sigma_{n}^{2}}}\right)^{R} \int e^{\frac{-\|y-h x\|^{2}}{2 \sigma_{n}^{2}}} \log _{2} \frac{\left(\frac{1}{\sqrt{2 \pi \sigma_{n}^{2}}}\right)^{R} e^{\frac{-\|y-h x\|^{2}}{2 \sigma_{n}^{2}}}}{p\left(y ; \mathrm{Q}\left(x^{*}\right)\right)} \mathrm{d} y \\
= & \lambda^{*}\left(P-\left\|\mathbf{x}^{*}\right\|^{2}\right)-R \log _{2} \sqrt{2 \pi e \sigma_{n}^{2}} \\
& -\left(\frac{1}{\sqrt{2 \pi \sigma_{n}^{2}}}\right)^{R} \int e^{\frac{-\|y-h x\|^{2}}{2 \sigma_{n}^{2}}} \log _{2} p\left(y ; \mathrm{Q}\left(x^{*}\right)\right) \mathrm{d} y .
\end{aligned}
$$

Hermite polynomials combination is used to eapand the last integration $[87,88]$. 


\subsubsection{The Optimal Input}

Depending on the whole discussions above, the following Lemma holds.

Lemma 7. For an average power limited finite-capacity memoryless channel subject to additive white Gaussian noise (5.1), the capacity-achieving input distribution $p\left(x^{*}\right)$ is Gaussian.

The remainder of the section is dedicated to the proof of the Lemma.

Let $p(y)=p\left(y ; \mathrm{Q}\left(x^{*}\right)\right)$ and by assuming that $\sigma_{n}=1$ without losing the generality

$$
\begin{aligned}
\mathrm{C}= & \lambda^{*}\left(P-\left\|\mathbf{x}^{*}\right\|^{2}\right)-R \log _{2} \sqrt{2 \pi e} \\
& -\left(\frac{1}{\sqrt{2 \pi}}\right)^{R} \int e^{\frac{-\|y\|^{2}}{2}} e^{\frac{-\left\|h x^{*}\right\|^{2}}{2}+\left\|h x^{*}\right\| y^{\mathrm{H}} \log _{2} p(y) \mathrm{d} y .}
\end{aligned}
$$

According to Hermite polynomial properties (Appendix C), the generating function is

$$
e^{\frac{-\left\|h x^{*}\right\|^{2}}{2}+\left\|h x^{*}\right\| y^{\mathrm{H}}}=\sum_{n=0}^{\infty} \mathrm{H}_{n}(y) \frac{\left\|h x^{*}\right\|^{n}}{n !} .
$$

Since $p(y)$ is a continuous function of $y, \log _{2} p(y)$ is also continuous and can be written as

$$
\log _{2} p(y)=\sum_{n=0}^{\infty} \mathrm{c}_{n} \mathrm{H}_{n}(y)
$$

By substitution, we get

$$
\begin{aligned}
& \left(\frac{1}{\sqrt{2 \pi}}\right)^{R} \int e^{\frac{-\|y\|^{2}}{2}} e^{\frac{-\left\|h x^{*}\right\|^{2}}{2}}+\left\|h x^{*}\right\| y^{\mathrm{H}} \log _{2} p(y) \mathrm{d} y \\
& =\left(\frac{1}{\sqrt{2 \pi}}\right)^{R-1} \int \frac{1}{\sqrt{2 \pi}} e^{\frac{-\|y\|^{2}}{2}} \sum_{n=0}^{\infty} \mathrm{H}_{n}(y) \frac{\left\|h x^{*}\right\|^{n}}{n !} \log _{2} p(y) \mathrm{d} y \\
& =\left(\frac{1}{\sqrt{2 \pi}}\right)^{R-1} \sum_{n=0}^{\infty}\left[\frac{1}{\sqrt{2 \pi} n !} \int e^{\frac{-\|y\|^{2}}{2}} \log _{2} p(y) \mathrm{H}_{n}(y) \mathrm{d} y\right]\left\|h x^{*}\right\|^{n} \\
& =\left(\frac{1}{\sqrt{2 \pi}}\right)^{R-1} \sum_{n=0}^{\infty} \mathrm{c}_{n}\left\|h x^{*}\right\|^{n} .
\end{aligned}
$$


Therefore

$$
\mathrm{C}=\lambda^{*}\left(P-\left\|\mathbf{x}^{*}\right\|^{2}\right)-R \log _{2} \sqrt{2 \pi e}-\left(\frac{1}{\sqrt{2 \pi}}\right)^{R-1} \sum_{n=0}^{\infty} \mathrm{c}_{n}\left\|h x^{*}\right\|^{n} .
$$

Or

$$
\sum_{n=0}^{\infty} \mathrm{c}_{n}\left\|h x^{*}\right\|^{n}=(\sqrt{2 \pi})^{R-1}\left[\lambda^{*}\left(P-\left\|\mathbf{x}^{*}\right\|^{2}\right)-\mathrm{C}-R \log _{2} \sqrt{2 \pi e}\right]
$$

By identifying the polynomials of the last equation, one can determine first the values of the coefficients $\mathrm{c}_{n}$ and then $p(y)$. This identification yields

$$
\begin{aligned}
& \mathrm{c}_{0}=(\sqrt{2 \pi})^{R-1}\left[\lambda^{*} P-\mathrm{C}-R \log _{2} \sqrt{2 \pi e}\right], \\
& \mathrm{c}_{1}=0, \\
& \mathrm{c}_{2} \leqslant-(\sqrt{2 \pi})^{R-1} \frac{\lambda^{*}}{\|h\|^{2}}, \text { and } \\
& \mathrm{c}_{n}=0 \quad \text { for } n \geqslant 3 .
\end{aligned}
$$

Consequently,

$$
\begin{aligned}
\log _{2} p(y) & =\sum_{n=0}^{\infty} \mathrm{c}_{n} \mathrm{H}_{n}(y) \\
& =\mathrm{c}_{0} \mathrm{H}_{0}(y)+\mathrm{c}_{2} \mathrm{H}_{2}(y) \\
& =\mathrm{c}_{0}+\mathrm{c}_{2}\left(\|y\|^{2}-1\right) \\
& =\left(\mathrm{c}_{0}-\mathrm{c}_{2}\right)+\mathrm{c}_{2}\|y\|^{2} .
\end{aligned}
$$

Hence

$$
\begin{aligned}
p(y) & =e^{\ln 2\left[\left(\mathrm{c}_{0}-\mathrm{c}_{2}\right)+\mathrm{c}_{2}\|y\|^{2}\right]} \\
& =\mathrm{k} e^{\mathrm{c}_{2} \ln 2\|y\|^{2}}, \text { where } \mathrm{k}=e^{\left(\mathrm{c}_{0}-\mathrm{c}_{2}\right) \ln 2} .
\end{aligned}
$$

The appropriate input probability law $p\left(x^{*}\right)$ that induces such a Gaussian distributed 
output could be found as

$$
\begin{aligned}
p(y \mid \mathbf{H} x) & =\left(\frac{1}{\sqrt{2 \pi}}\right)^{R} e^{\frac{-\|y-h x\|^{2}}{2}} \quad\left(\sigma_{n}=1\right) \\
& =\left(\frac{1}{\sqrt{2 \pi}}\right)^{R} e^{\frac{-\|y\|^{2}}{2}} e^{\frac{-\left\|h x^{*}\right\|^{2}}{2}}+\left\|h x^{*}\right\| y^{\mathrm{H}} \\
& =\left(\frac{1}{\sqrt{2 \pi}}\right)^{R} e^{\frac{-\|y\|^{2}}{2}} \sum_{n=0}^{\infty} \mathrm{H}_{n}(y) \frac{\left\|h x^{*}\right\|^{n}}{n !}
\end{aligned}
$$

then

$$
\begin{aligned}
p\left(y ; \mathrm{Q}\left(x^{*}\right)\right) & =\int p(y \mid \mathbf{H} x) \mathrm{dQ}\left(x^{*}\right) \\
& =\int\left(\frac{1}{\sqrt{2 \pi}}\right)^{R} e^{\frac{-\|y\|^{2}}{2}} \sum_{n=0}^{\infty} \mathrm{H}_{n}(y) \frac{\left\|h x^{*}\right\|^{n}}{n !} \mathrm{dQ}\left(x^{*}\right) \\
& =p(y) \\
& =\mathrm{k} e^{\mathrm{c}_{2} \ln 2\|y\|^{2}}, \quad \text { due (5.25). }
\end{aligned}
$$

Hence

$$
\mathrm{k} e^{\mathrm{c}_{2} \ln 2\|y\|^{2}}=\left(\frac{1}{\sqrt{2 \pi}}\right)^{R-1} \sum_{n=0}^{\infty} \frac{1}{\sqrt{2 \pi} n !} \int e^{\frac{-\|y\|^{2}}{2}}\left\|h x^{*}\right\|^{n} \mathrm{dQ}\left(x^{*}\right) \mathrm{H}_{n}(y)
$$

Multiplying (5.28) by $\mathrm{H}_{n}(y)$ gives

$$
\begin{aligned}
& {\left[\mathrm{k} e^{\mathrm{c}_{2} \ln 2\|y\|^{2}}\right] \mathrm{H}_{n}(y)=} \\
& \left(\frac{1}{\sqrt{2 \pi}}\right)^{R-1} \sum_{n=0}^{\infty}\left[\frac{1}{\sqrt{2 \pi} n !} \int e^{\frac{-\|y\|^{2}}{2}}\left\|h x^{*}\right\|^{n} \mathrm{dQ}\left(x^{*}\right) \mathrm{H}_{n}(y)\right] \mathrm{H}_{n}(y) .
\end{aligned}
$$

The last result could be manipulated as the following:

$$
\int\left[\mathrm{k} e^{\mathrm{c}_{2} \ln 2\|y\|^{2}}\right] \mathrm{H}_{n}(y) \mathrm{d} y=\left(\frac{1}{\sqrt{2 \pi}}\right)^{R-1} \int\left\|h x^{*}\right\|^{n} \mathrm{dQ}\left(x^{*}\right) .
$$

The integration on the left hand side could be considered as the expectation operation of $\mathrm{H}_{n}(z)$ where $z$ is a complex random variable with normal distribution of zero mean and $\sigma_{z}$ standard deviation. 
Therefore

$$
\begin{aligned}
& \int\left[\mathrm{k} e^{\mathrm{c}_{2} \ln 2\|z\|^{2}}\right] \mathrm{H}_{n}(z) \mathrm{d} z=\mathrm{k} \sqrt{2 \pi \sigma_{z}^{2}} \int \mathrm{H}_{n}(z) \frac{e^{\frac{-\|z\|^{2}}{2 \sigma_{z}^{2}}}}{\sqrt{2 \pi \sigma_{z}^{2}}} \mathrm{~d} z \\
& =\mathrm{k} \sqrt{2 \pi \sigma_{z}^{2}} \mathrm{E}\left[\mathrm{H}_{n}(z)\right] \\
& =\mathrm{k} \sqrt{2 \pi \sigma_{z}^{2}} \mu^{n} \mathrm{H}_{n}(0), \quad \mu \text { is the expectation of } \mathrm{H}_{n}(z) \text {. } \\
& =\mathrm{k} \sqrt{2 \pi \sigma_{z}^{2}}\left(\sqrt{\mathrm{E}\left[z^{2}\right]-\sigma_{z}^{2}}\right)^{n} \mathrm{H}_{n}(0) \\
& =\mathrm{k} \sqrt{2 \pi \sigma_{z}^{2}}\left(\sqrt{(2-1) ! !-\sigma_{z}^{2}}\right)^{n} \mathrm{H}_{n}(0) \\
& =\mathrm{k} \sqrt{2 \pi \sigma_{z}^{2}}\left(\sqrt{1-\sigma_{z}^{2}}\right)^{n} \mathrm{H}_{n}(0) \\
& = \begin{cases}0 & \text { if } n \text { is odd } \\
\mathrm{k} \sqrt{2 \pi \sigma_{z}^{2}}\left(\sqrt{1-\sigma_{z}^{2}}\right)^{n} \mathrm{H}_{n}(0) & \text { if } n \text { is even, }\end{cases}
\end{aligned}
$$

where

$$
\sigma_{z}^{2}<\frac{\|h\|^{2}}{(\sqrt{2 \pi})^{R-1} \lambda^{*} 2 \ln 2} .
$$

Hence

$$
\left(\frac{1}{\sqrt{2 \pi}}\right)^{R-1} \int\left\|h x^{*}\right\|^{n} \mathrm{dQ}\left(x^{*}\right)=\mathrm{k} \sqrt{2 \pi \sigma_{z}^{2}}\left(\sqrt{1-\sigma_{z}^{2}}\right)^{n} \mathrm{H}_{n}(0) .
$$

Or

$$
\int\left\|h x^{*}\right\|^{n} \mathrm{dQ}\left(x^{*}\right)=\mathrm{k} \sqrt{(2 \pi)^{R} \sigma_{z}^{2}}\left(\sqrt{1-\sigma_{z}^{2}}\right)^{n} \mathrm{H}_{n}(0) .
$$

The last integral is kind of Riemann-Stieltjes integral applies to probability theory. It represents the $n$-th moment of the probability distribution. Thus, the moment 
generating function $(\mathrm{MGF}), \mathrm{M}_{x^{*}}(t)$, is

$$
\begin{aligned}
\mathrm{M}_{x^{*}}(t) & =\int\left\|h x^{*}\right\|^{n} \mathrm{dQ}\left(x^{*}\right) \\
& =\mathrm{E}\left[e^{t\left\|h x^{*}\right\|}\right] \\
& =\sum_{x^{*}} e^{t\left\|h x^{*}\right\|} \mathrm{k} \sqrt{(2 \pi)^{R} \sigma_{z}^{2}}\left(\sqrt{1-\sigma_{z}^{2}}\right)^{n} \mathrm{H}_{n}(0) \\
& =\sum_{x^{*}} \sum_{n=0}^{\infty} \frac{\left(t\left\|h x^{*}\right\|\right)^{n}}{n !} \mathrm{k} \sqrt{(2 \pi)^{R} \sigma_{z}^{2}}\left(\sqrt{1-\sigma_{z}^{2}}\right)^{n} \mathrm{H}_{n}(0) \\
& =\mathrm{k} \sqrt{(2 \pi)^{R} \sigma_{z}^{2}} \sum_{n=0}^{\infty}[\mathrm{H}_{n}(0) \frac{\left(\sqrt{1-\sigma_{z}^{2}} t\right)^{n}}{n !} \underbrace{\sum_{x^{*}}\left\|h x^{*}\right\|}_{=1 \text { for } n=0}] \\
& =\mathrm{k} \sqrt{(2 \pi)^{R} \sigma_{z}^{2}} e^{-\frac{1-\sigma_{z}^{2}}{2} t^{2}} .
\end{aligned}
$$

Consequently, it is obvious that the PDF of the continuous input $x^{*}$ is

$$
p\left(x^{*}\right)=\mathrm{k} \sqrt{(2 \pi)^{R} \sigma_{z}^{2}} e^{-\frac{1-\sigma_{z}^{2}}{2}\left\|x^{*}\right\|^{2}},
$$

which is valid whenever $\sigma_{z}^{2} \leqslant 1$ or

$$
\lambda^{*} \geqslant \frac{\|h\|^{2}}{(2 \pi)^{R-1} 2 \ln 2} .
$$

\subsection{Capacity Under Amplitude-Limited Inputs}

In the following, we concentrate on deriving the optimal power allocation strategy to achieve the maximum under the availability of channel information. For a given average transmit power over $T$ antennas, the optimization analysis discussed in the last section could be accomplished here assuming bounded range of symbol gain $(s)$ for an M-QAM set. Clearly, we now need to maximize I $(\mathbf{x} ; \mathbf{y})$ for all $s>0$. To do this, we must find the largest $s$, referred to $s^{*}$, such that the code still converges to zero error rate. Such $s^{*}$ could be found through binary search, noting that it is possible to limit the search space to

$$
s_{\min } \leqslant s \leqslant s_{\max },
$$


where $s_{\min }$ and $s_{\max }$ are the minimum and maximum respectively.

Lemma 8. There exists a bounded range for M-QAM signal constellation gains ( $s$ ) such that

$$
\sqrt{\frac{P}{2(\sqrt{\mathrm{M}}-1)^{2} T}}=s_{\min } \leqslant s \leqslant s_{\max }=\sqrt{\frac{P}{2 T}},
$$

where $P$ is transmitting power over $T$ antennas.

Proof. At the beginning, it is necessary to show how could the power constraint given by $s^{2} \mathrm{E}\left[\left\|x_{i}\right\|^{2}\right] \leqslant P, \quad \forall x_{i} \in$ M-QAM be replaced with equality (without loss of optimality). For the two scaling factors $s_{1}>0$ and $s_{2}>0$, assume that $\forall x_{i} \in$ M-QAM

$s_{1}^{2} \mathrm{E}\left[\left\|x_{i}\right\|^{2}\right] \leqslant P, \quad$ and $s_{2}^{2} \mathrm{E}\left[\left\|x_{i}\right\|^{2}\right]=P$.

It is being adequate to say that $\mathrm{I}\left(\mathrm{x} ; \mathrm{y}_{1}\right) \geqslant \mathrm{I}\left(\mathrm{x} ; \mathrm{y}_{2}\right)$ with $y_{1}$ and $y_{2}$ are the corresponding output random variables.

Thus,

$$
\begin{array}{rlrl}
\mathrm{I}\left(\mathrm{x} ; \mathrm{y}_{1}\right) & =h(\mathrm{x})-h\left(\mathrm{x} \mid \mathrm{y}_{1}\right) & \\
& \leqslant h(\mathrm{x})-h\left(\mathrm{x} \mid \mathrm{y}_{2}, \mathrm{y}_{1}\right) & \text { conditioning on an extra variable can decrease entropy } \\
& =h(\mathrm{x})-h\left(\mathrm{x} \mid \mathrm{y}_{2}\right) & p_{\mathrm{x} \mid \mathrm{yz}}(x \mid y, z)=p_{\mathrm{x} \mid \mathrm{y}}(x \mid y) \\
& =\mathrm{I}\left(\mathrm{x} ; \mathrm{y}_{2}\right) .
\end{array}
$$

Hence, $s^{2} \sum_{x_{i} \in \mathcal{X}_{\mathrm{M}-\mathrm{QAM}}}\left\|x_{i}\right\|^{2}=P$.

Secondly, in many $\mathcal{X}_{\mathrm{M}-\mathrm{QAM}}$ constellations, the power requires to transmit the highestamplitude symbol is $P_{\max }=2(\sqrt{\mathrm{M}}-1)^{2}$, and for $T$ transmit antennas it is

$$
P_{\max }=2(\sqrt{\mathrm{M}}-1)^{2} T
$$

where $\mathrm{M}=2^{m}$.

While the peak needs power approximately tends to vary with the number of $m$ as 
$P \propto 2^{m}$, then the minimum transmitted power over $T$ antennas is

$$
P_{\min }=2 T
$$

Consequently, from (5.39) and (5.40) we get

$$
s_{\max }=\sqrt{\frac{P}{P_{\min }}}=\sqrt{\frac{P}{2 T}} \text { and } \quad s_{\min }=\sqrt{\frac{P}{P_{\max }}}=\sqrt{\frac{P}{2(\sqrt{\mathrm{M}}-1)^{2} T}} .
$$

Algorithms 1 and 2 present in details an iterative procedure to perform the MQAM capacity computation problem.

\section{Algorithm $1 s^{*}, p\left(x^{*}\right)$, and $\mathrm{C}_{\mathrm{M}-\mathrm{QAM}}$ Calculations.}

1: Set $s=s_{\min }$.

2: For all $x \in \mathcal{X}_{\mathrm{M}-\mathrm{QAM}}$, compute

- $\mathrm{C}=\sup _{\mathrm{Q} \in \mathcal{Q}} \iint p(y \mid \mathbf{H} x) \log _{2} \frac{p(y \mid \mathbf{H} x)}{p(y ; \mathrm{Q}(x))} \mathrm{d} y \mathrm{dQ}(x), p(y ; \mathrm{Q}(x))=\mathrm{k} e^{\mathrm{c}_{2} \ln 2\|y\|^{2}}$.

$$
\begin{aligned}
& \text { - } p(x)=\mathrm{k} \sqrt{(2 \pi)^{R} \sigma_{z}^{2}} e^{-\frac{1-\sigma_{z}^{2}}{2}\|s x\|^{2}}, \mathrm{k}=e^{\left(\mathrm{c}_{0}-\mathrm{c}_{2}\right) \ln 2}, \mathrm{c}_{2} \leqslant-(\sqrt{2 \pi})^{R-1} \frac{\lambda}{\|h\|^{2}} \\
& \mathrm{c}_{0}=(\sqrt{2 \pi})^{R-1}\left[\lambda P-\mathrm{C}-R \log _{2} \sqrt{2 \pi e}\right] \text { and } \sigma_{z}^{2}<\frac{\|h\|^{2}}{(\sqrt{2 \pi})^{R-1} \lambda 2 \ln 2} .
\end{aligned}
$$

3: $\lambda$ is chosen to satisfy

$$
\begin{aligned}
& \text { - } \lambda>\frac{-\log _{2}[p(y ; \mathrm{Q}(x))]-\mathrm{C}}{\|s x\|^{2}-P}, \text { and } \\
& \text { - } \lambda \geqslant \frac{\|h\|^{2}}{(2 \pi)^{R-1} 2 \ln 2} .
\end{aligned}
$$

4: Repeat steps $2 \& 3$. When $p(x)$ does converge, call it $p\left(x^{*}\right)$.

5: Set $s=s_{\text {new }}$ (as in Algorithm 2). Go to step 2.

6: Compute $\mathrm{C}_{\mathrm{M}-\mathrm{QAM}}=\int p(y \mid \mathbf{H} x) \log _{2} \frac{p(y \mid \mathbf{H} x)}{p\left(y ; \mathrm{Q}\left(x^{*}\right)\right)} \mathrm{d} y$. 


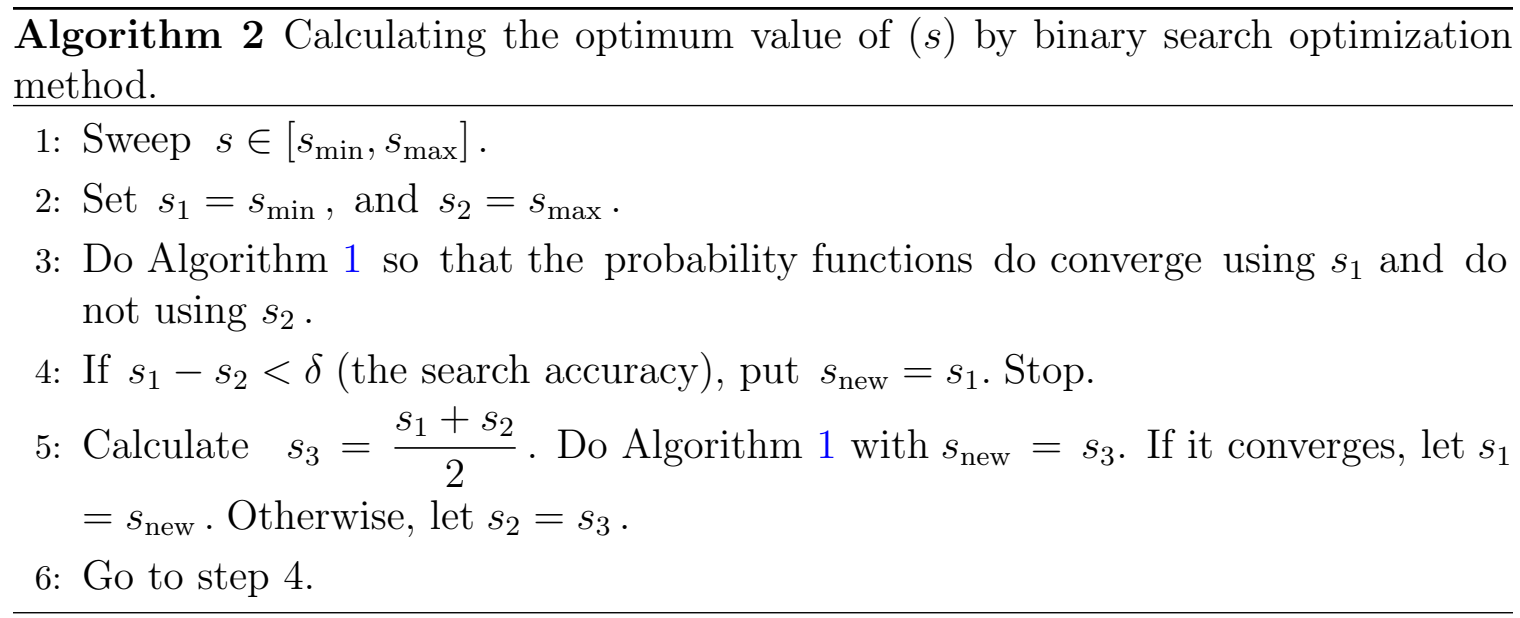

\subsection{Factorizing the Optimal QAM Distribution}

The system with $\left(\mathrm{M}^{T}-1\right)$ optimized variables over $\mathcal{X}_{\mathrm{M}-\mathrm{QAM}}$ constellation gets extremely high complexity and needs a solution that simplifies such presented algorithm (Algorithm 1). This solution focuses on the idea that QAM constellation could be performed of signal points placed symmetrically in (I-Q) plane and gives an amplitude-modulation of two orthogonal waveforms. Thus,

$$
\mathcal{X}_{\mathrm{M}-\mathrm{QAM}}=\mathcal{X}_{\sqrt{\mathrm{M}-\mathrm{PAM}}} \times \mathcal{X}_{\sqrt{\mathrm{M}-\mathrm{PAM}}}=\mathcal{X}_{\sqrt{\mathrm{M}-\mathrm{PAM}}}^{2}
$$

And

$$
\mathbf{x}=\left(x_{1 i}, x_{1 q}, x_{2 i}, x_{2 q}, \ldots, x_{\mathrm{M} i}, x_{\mathrm{M} q}\right) \in \mathcal{X}_{\sqrt{\mathrm{M}-\mathrm{PAM}}}^{2} \cdot
$$

We will denote $q(x)$ to be any valid probability function defined over $\mathcal{X}_{\sqrt{\mathrm{M}} \text {-PAM }}$. If all of the elements in (5.42) are supposed to be i.i.d. with $q(x)$, this means we are actually looking up for the distribution $q\left(x^{*}\right)$ that maximizes $\mathrm{C}$.

Accordingly, we make the following important conjectures.

Conjecture 1. The input distribution $p\left(x^{*}\right)$ that could maximize $\mathrm{C}$ over $\mathcal{X}_{\mathrm{M}-\mathrm{QAM}}$ realizes as

$$
p\left(x^{*}\right)=\prod_{m \in\{i, q\}} \prod_{n=1}^{T} q\left(x_{m n}^{*}\right) \quad \forall x_{m n}^{*} \in \mathcal{X}_{\sqrt{\mathrm{M}} \text {-PAM }}
$$

Conjecture 2. If $\boldsymbol{x}=\left(x_{1 i}, x_{1 q}, x_{2 i}, x_{2 q}, \ldots, x_{M i}, x_{M q}\right)$ and $p(x)$ has the form of (5.43), then

$$
\mathrm{E}\left[x_{m n}^{2}\right] \leqslant \frac{P}{2 T}
$$


As a result, Algorithm 3 presents the procedure for accomplishing this optimization task.

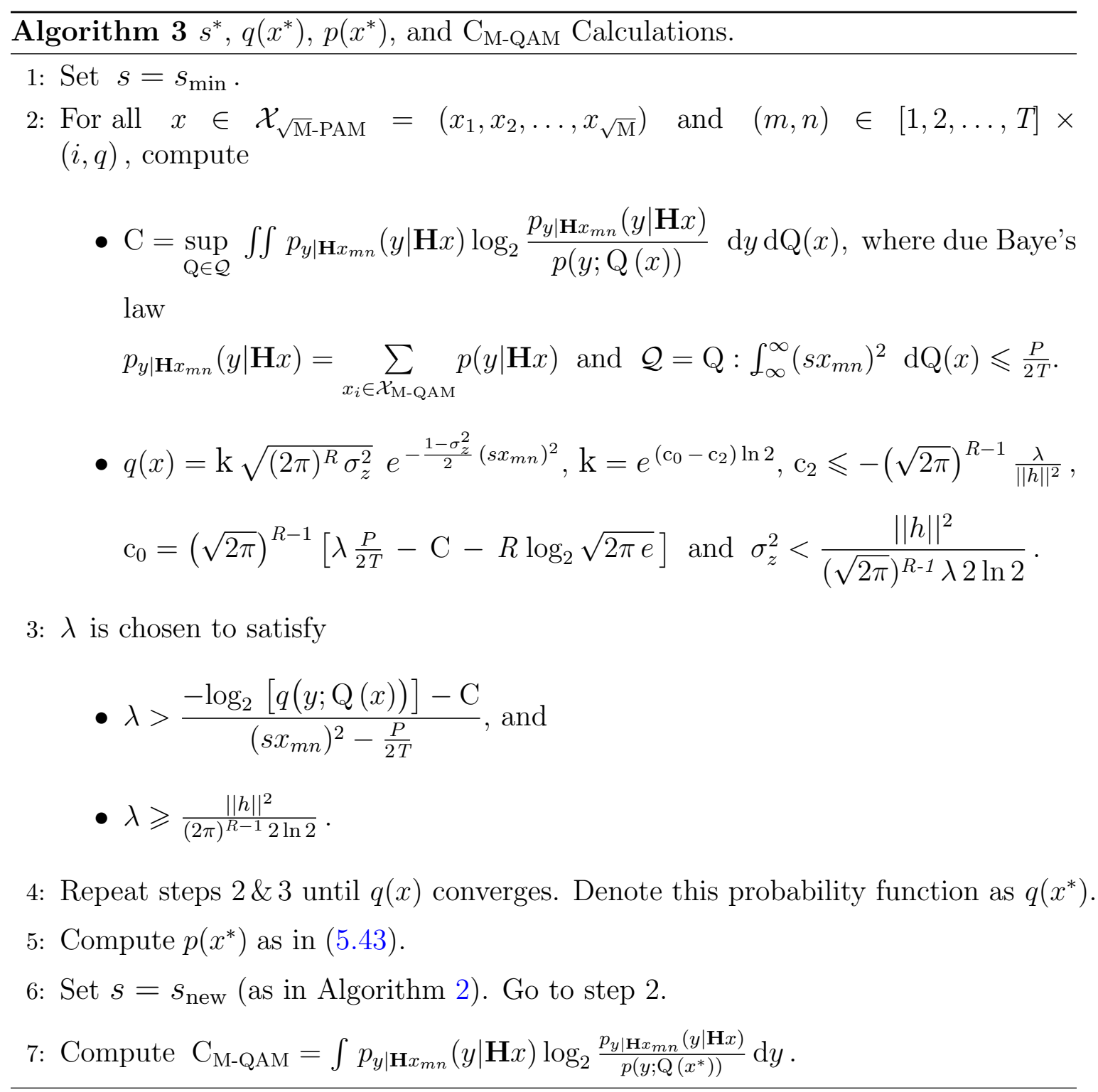

Finally, to examine the results, $\mathrm{C}_{\mathrm{M}-\mathrm{QAM}}$ is compared with the information rate achieved using independent and uniformly distributed (i.u.d) transmission $\left(\mathrm{C}_{\mathrm{M}-\mathrm{QAM} \text {-i.u.d. }}\right)$. We consider all signal points are equiprobable and therefore the constellation gain $s$ sets to $\sqrt{\frac{P}{\frac{\mathrm{M}^{2}-1}{12}}}$ and $p\left(x_{i}\right)=\frac{1}{\mathrm{M}^{T}}, i \in\left[1,2, \ldots, \mathrm{M}^{\mathrm{T}}\right]$. 


\subsection{Numerical Results}

Algorithm 3 is used to compute the achievable rate under Rayleigh fading channel with SC polar codes of 1024 code length and rate of $\frac{1}{2}$ coded the 16-QAM input signal constellation for a $(2 \times 2)$ system. For example, in Fig. 5.2 we can see that a rate of 1.3 bit per channel used is achieved at $9.298 \mathrm{~dB}$ when $\mathcal{X}_{\mathrm{M}-\mathrm{QAM}}$ constellation is transmitted over each antenna. By using $\mathcal{X}_{\sqrt{16} \text {-PAM }}^{2}$ constellation, this rate could be achieved at $9.357 \mathrm{~dB}$ by utilizing an optimum constellation gain of $s^{*}=0.5825$. Thus, $\mathrm{C}_{\mathrm{M}-\mathrm{QAM}}$ is within 0.059 $\mathrm{dB}$ from channel capacity limit at 1.3 bit per channel used; while $\mathrm{C}_{\mathrm{M} \text {-QAM-i.u.d. }}$ is about $0.349 \mathrm{~dB}$ from the limit, displaying a gap of $0.29 \mathrm{~dB}$ with $\mathrm{C}_{\mathrm{M}-\mathrm{QAM}}$.

Now, according to Algorithm 1 there are $16^{2}-1=255$ variables required to fulfill the optimization task; compared with $\sqrt{16}-1=3$ carried out according to Algorithm 3 .

Fig. 5.3 presents the numerically computed rates when Gaussian distribution is

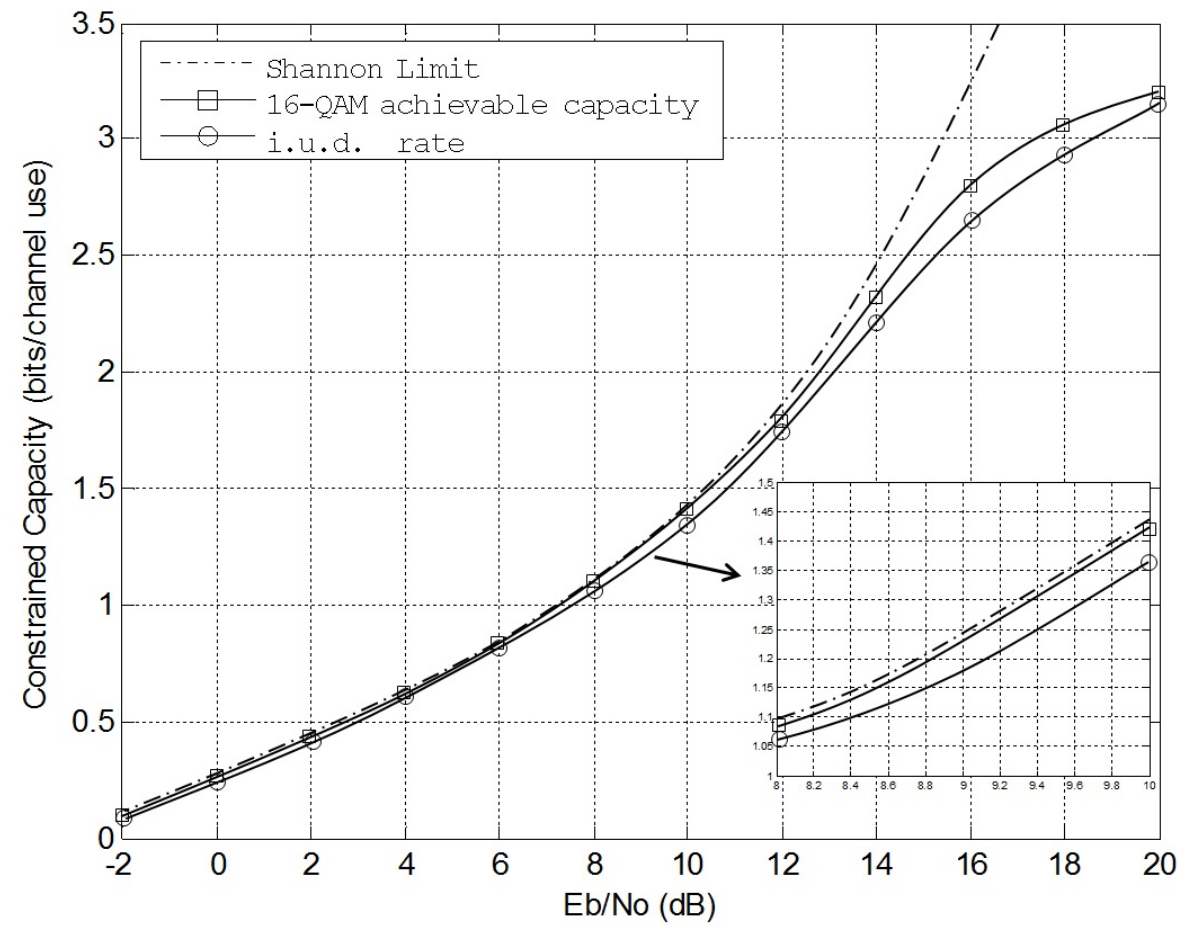

Figure 5.2: Achieved capacities under different transmission scenarios. The SC polar codes has code length of 1024 with half rate. 


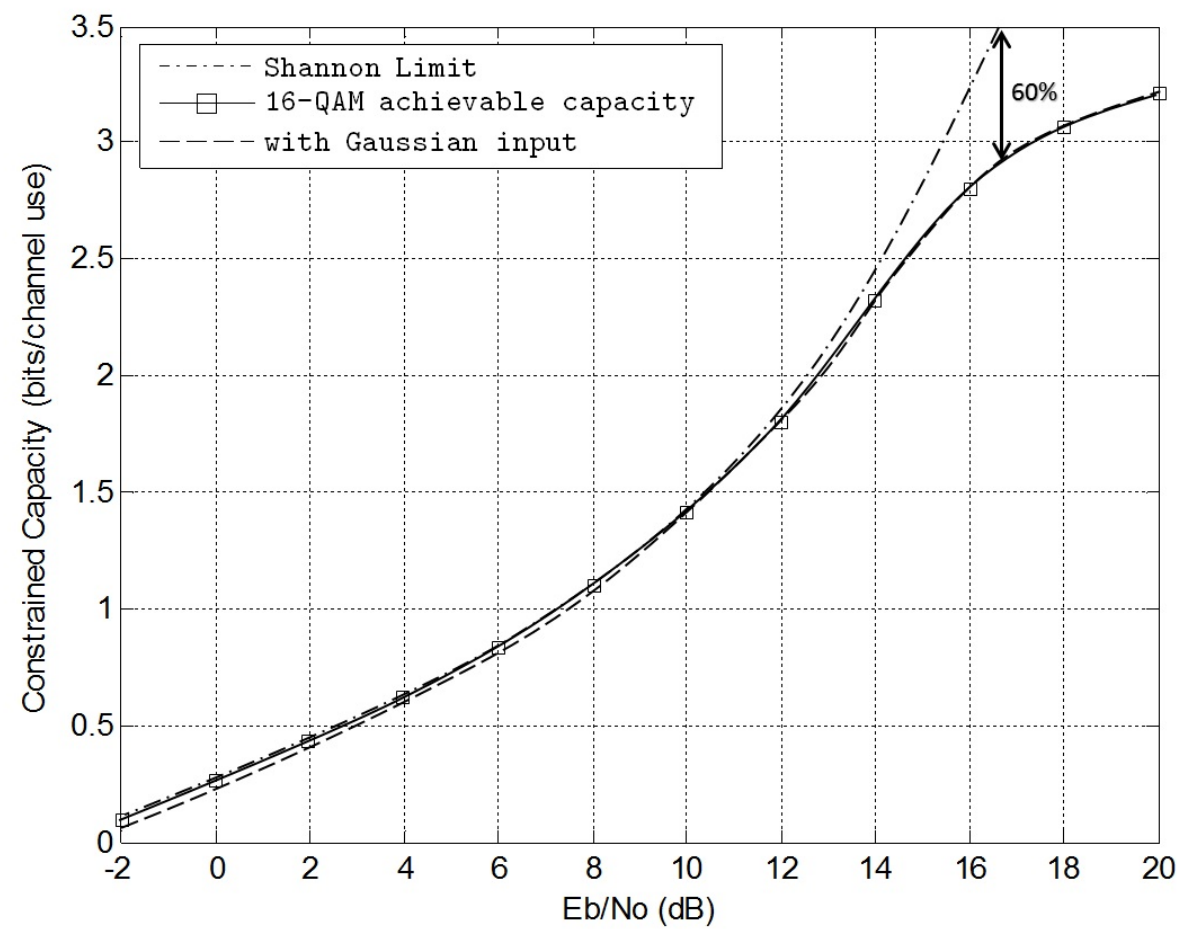

Figure 5.3: Achieved capacities under different transmission scenarios. The SC polar codes has code length of 1024 with half rate.

employed. It is shown that the use of the continuous optimal input can performs better than the Gaussian one with the fading channel. Also it is observed the cumulative detrimental effect of such channels linearity yields a rate lower as compared to Shannon limit by $60 \%$ at $16.7 \mathrm{~dB}$. Such rates degradation is found to be for low dBs as $4 \%$ in maximum.

\subsection{Summary}

An optimization method is introduced in this chapter for approaching the capacity of fading channel with multi-antennas. Under 16-QAM modulation with the condition $\mathrm{E}\left[x^{H} x\right] \leqslant P$ and the aid of Hermite polynomials, an optimum Gaussian distribution with its correction factor are determined. Moreover, we have proved that the complexity of the proposed algorithm could be much reduced by factoring the determined Gaussian distribution into a couple of 4-PAM signals. 


\section{Chapter 6}

\section{Conclusions and Future Work}

As discussed in Chapter 1 and Chapter 2, polar codes open a new door for reliable data transmission since they can achieve the capacity of B-DMCs with low encoding and decoding complexity. We have presented several information-theoretic results for polar codes and discussed some methods to improve the performance and computational complexity of polar codes.

In this chapter, we summarize all the contributions of this thesis and then discuss a few directions in which this work can be extended.

In Chapter 3, we reviewed both the MS and SP decoding algorithms. We then analyzed the corrected MS algorithm and concluded that compared to the optimum SP algorithm, its check node processing always produces outputs with the same signs but larger magnitudes. Thus we addressed this problem to improve the check node processing of the corrected MS algorithm by normalization. The simulation results show that with proper normalization factors, the corrected NMS algorithm can achieve near optimum decoding performance. Then we introduced an approach to determine the best normalization factors by DE technique; and with the derived formulas, we calculated the best possible asymptotic performance of the corrected NMS algorithm for the corresponding normalization factors.

A natural extension for future research is:

- To overcome the problem when $\operatorname{sgn}\left(\lambda_{0}\right) \neq \operatorname{sgn}\left(\lambda_{1}\right)$, or they are equal in some circumstances. Generally, we can identify three cases: 1) $\operatorname{sgn}\left(\lambda_{0}\right) \neq \operatorname{sgn}\left(\lambda_{1}\right)$; 2) $\operatorname{sgn}\left(\lambda_{0}\right)=\operatorname{sgn}\left(\lambda_{1}\right), \quad\left|\lambda_{0}\right|<\left|\lambda_{1}\right|$; and 3) $\operatorname{sgn}\left(\lambda_{0}\right)=\operatorname{sgn}\left(\lambda_{1}\right), \quad\left|\lambda_{0}\right| \geqslant\left|\lambda_{1}\right|$. Obviously, a number of simplifications need to be postulated in order to solve the problem. 
- To focus on SCL decoding. While the method proposed in Chapter 3 improve error-correction performance, it is less effective at doing so than the SCL algorithm. One avenue to pursue would be to increase parallelism beyond what current hardware implementations offer. Another would be to investigate more efficient memory sharing techniques to enable low-complexity decoders with a large list size.

- To devise reduced complexity decoding algorithms for non-binary polar codes.

- VLSI implementation of polar decoders (SC or SCL) with the corrected NMS algorithm.

In Chapter 4, the issue of improving the performance of SC polar decoders based on suboptimal calculation of the messages exchanged during the check node processing (LLR values) is addressed. It is well known in the literature that when CSI is not available at the receiver then a simple, yet very effective, way to improve the performance of suboptimal decoders is based on applying a linear approximation to the LLR values. Starting with a theoretical model based on the so-called consistency condition of a random variable, we suggest a methodology for correcting the LLR values that relies only on the distribution of the soft information exchanged in the check node process. From the viewpoint of channel capacity, this methodology gives a clear explanation of why the well-known linear approximation provides a very good performance. Additionally, a generic method to calculate the channel capacity of BPSK modulated Rayleigh fading channel subjected to non-uniform soft quantization is proposed, which offers a powerful criterion for optimal quantizer design. Numerical simulations show that for polar codes the approximated values found closely follow the true values, which translates to a close-to-optimal BER performance. Moreover, a 6bit uniform quantization and also a uniform quantization offer effective demodulation compared with unquantized one, losing less than $0.07 \mathrm{~dB}$ for both with and without CSI cases.

An interesting open problem is:

- To facilitate good LLR approximations for non-binary modulations (16-QAM for example) as the LLR computation for non-binary modulations over fading channels is complicated.

- To achieve more reduction in hardware complexity for LUT implementation by updating equation (4.16). Then number of LUT entries should be reduced 
accordingly which offers more hardware reduction compared to uniform quantization scheme.

In Chapter 5, we investigate the detailed characterization of the capacityachieving input signals for transmission over a wireless multiple antenna communication system operating in a Rayleigh flat-fading channel with Gaussian noise where both the transmitter and the receiver has the knowledge of fading coefficients. We obtain necessary and sufficient conditions for the capacity-achieving input distribution and show that, subject to the average power constraint, this distribution is unique input with Gaussian distribution that achieves the channel capacity. The optimal input distribution that achieves the channel capacity is derived through a methodology based on Hermite polynomials as a basis of expansions of various functions. The results also show that the computational complexity can be reduced by factorizing the optimal distribution into the product of symmetrical distributions.

Below is a few directions in which our work can be extended:

- Throughout the derivation of the optimal input (Subsection 5.2.2), $\sigma_{n}$ is assumed to be 1 which helps in getting a solution for the integral. This integral (5.13) may still be generalize for any value of $\sigma_{n}$.

- To address variety of memoryless nonlinear channels where the input of the channel is distorted according to some deterministic function $f(\mathrm{x})$ of the form: 1) $\left.f(\mathrm{x})=\mathrm{x}^{n}, n \in \mathbb{N} \backslash\{1\}, 2\right) f(\mathrm{x})=|\mathrm{x}|^{n}$, or 3) $f(\mathrm{x})=|\mathrm{x}|^{\frac{1}{n}}, n \in \mathbb{N} \backslash\{1\}$.

- To address the problem of power allocation to maximize average capacity subject to multiple power constraints for perfect CSI at both the transmitter and the receiver.

- To investigate the communication limits over rapid phase-varying channels and consider the capacity of a non (or partially) coherent AWGN channel under the average power constraint.

- An interesting direction is to extend this work for channels such as multiple access channels, broadcast channels and interference channels. 


\section{List of References}

[1] E. Arikan, "Channel polarization: A method for constructing capacity-achieving codes for symmetric binary-input memoryless channels," IEEE Transactions on Communications, vol. 55, no. 07, pp. 3051-3073, July 2009.

[2] D. Muller, "Application of boolean algebra to switching circuit design and to error detection," IRE Professional Group on Electronic Computers Transactions on Information Theory, vol. EC-3, no. 03, pp. 6-12, September 1954.

[3] I. Reed, "A class of multiple-error-correcting codes and the decoding scheme," IRE Professional Group on Electronic Computers Transactions on Information Theory, vol. 04, no. 04, pp. 38-49, September 1954.

[4] I. Tal and A. Vardy, "List decoding of polar codes," IEEE Transactions on Information Theory, vol. 61, no. 5, pp. 2213-2226, May 2015.

[5] U. Fayyaz and J. Barry, "Low-complexity soft-output decoding of polar codes," IEEE Journal on Selected Areas in Communications, vol. 32, no. 05, pp. 958-966, May 2014.

[6] A. Stimming, M. Parizi, and A. Burg, "LLR-based successive cancellation list decoding of polar codes," IEEE Transactions on Communications, vol. 63, no. 19, pp. 5165-5179, October 2015.

[7] S. Hashemi, C. Condo, and W. Gross, "Simplified successive-cancellation list decoding of polar codes," IEEE International Symposium on Information Theory (ISIT), pp. 815-819, August 2016.

[8] S. Hashemi, C. Condo, and W. Gross, "Fast and flexible successive-cancellation list decoders for polar codes," IEEE Transactions on Signal Processing, vol. 65, no. 21, pp. 5756-5769, August 2017.

[9] S. Hashemi, A. Stimming, P. Giard, C. Thibeault, and W. Gross, "Partitioned successive-cancellation list decoding of polar codes," IEEE International Conference on Acoustics, Speech and Signal Processing (ICASSP), pp. 957-960, May 2016.

[10] F. Ercan, C. Condo, S. Hashemi, and W. Gross, "On error-correction performance and implementation of polar code list decoders for 5G," IEEE 55th Annual Allerton Conference on Communication, Control, and Computing (Allerton), pp. 443-449, January 2018. 
[11] B. Zhang et al, "A 5G trial of polar code," IEEE Globecom Workshops (GC Wkshps), pp. 01-06, December 2016.

[12] W. Wang et al, "Field trial on TDD massive MIMO system with polar code," IEEE International Symposium on Personal, Indoor, and Mobile Radio Communication (PIMRC), pp. 01-06, October 2017.

[13] C. Yaacoub and M. Sarkis, "Systematic polar codes for joint source-channel coding in wireless sensor networks and the internet of things," International Conference on Future Networks and Communications (FNC), pp. 266-273, 2017.

[14] S. Hashemi, A. Stimming, P. Giard, C. Thibeault, and W. Gross, "Fast polar decoders: algorithm and implementation," IEEE Journal on Selected Areas in Communications, vol. 32, no. 5, pp. 946-957, May 2014.

[15] Y. Park, Y. Tao, S. Sun, and Z. Zhang, "A 4.68Gb/s belief propagation polar decoder with bit-splitting register file," IEEE Symposium on VLSI Circuits Digest of Technical Papers, pp. 01-02, July 2014.

[16] A. Ren, B. Yuan, and Y. Wang, "Design of high-speed low-power polar BP decoder using emerging technologies," IEEE International System-on-Chip Conference $(S O C C)$, pp. 312-316, September 2016.

[17] A. Cassagne, O. Aumage, C. Leroux, D. Barthou, and B. Gal, "Energy consumption analysis of software polar decoders on low power processors," 24th European Signal Processing Conference (EUSIPCO), pp. 642-646, December 2016.

[18] J. Zarate and M. Dohler, "Machine-to-machine (M2M) Communications: Architecture, Performance and Applications," Woodhead Publishing, 1st Edition, January 2015.

[19] Dylan Bushell-Embling, [Online] Available: http://en.ofweek.com/news/Huaweiachieves-27Gbps-5G-speeds-with-Polar-Code-42760.

[20] A. Hasan and I. Marsland, "SC of polar codes based normalized MS algorithm," IEEE Annual Information Technology, Electronics and Mobile Communication Conference (IEMCON), pp. 111-116, October 2017.

[21] A. Hasan and I. Marsland, "Performance of NMS decoding of SC polar codes based density evolution," IEEE Annual Information Technology, Electronics and Mobile Communication Conference (IEMCON), pp. 95-100, October 2017.

[22] A. Hasan and I. Marsland, "Corrected NMS-SC decoding of polar codes," under review in IEEE Transactions on Communications, April 2018.

[23] A. Hasan and I. Marsland, "Channel optimization and LLR approximation based SC of polar codes," IEEE Annual Information Technology, Electronics and Mobile Communication Conference (IEMCON), pp. 117-120, October 2017.

[24] A. Hasan and I. Marsland, "Non-uniform quantizers with SC polar and channeloptimized decoders," IEEE Annual Information Technology, Electronics and Mobile Communication Conference (IEMCON), pp. 101-104, October 2017. 
[25] A. Hasan and I. Marsland, "On non-uniform quantizers for SC polar decoders over fading channels," under review in IEEE Transactions on Communications, April 2018.

[26] A. Hasan and I. Marsland, "On optimal signalling distribution of SC Polar decoding based MIMO fading channels," IEEE Annual Information Technology, Electronics and Mobile Communication Conference (IEMCON), pp. 105-110, October 2017.

[27] A. Hasan and I. Marsland, "Low computational complexity of SC polar decoder in MIMO fading channel," IEEE Future Technologies Conference (FTC) Proceeding, November 2017.

[28] A. Hasan and I. Marsland, "On the capacity-achieving distribution of the coherent MIMO Rayleigh fading channels," under review in IEEE Transactions on Communications, April 2018.

[29] A. Hasan and I. Marsland, "Low complexity LLR metrics for polar coded QAM," IEEE Canadian Conference on Electrical and Computer Engineering (CCECE), pp. 01-04, June 2017.

[30] U. Fayyaz and J. Barry, "Polar codes design and decoding for magnetic recording," PhD dissertation, Georgia Institution of Technology, December 2014.

[31] J. Guo, A. Fábregas, and J. Sayir, "Fixed-threshold polar codes," IEEE International Symposium on Information Theory (ISIT), pp. 947-951, October 2013.

[32] J. Guo, M. Qin, A. Fábregas, and P. Siegel, "Enhanced belief propagation decoding of polar codes through concatenation," IEEE International Symposium on Information Theory (ISIT), pp. 2987-2991, August 2014.

[33] J. Guo and A. Fábregas, "Polar codes for reliable transmission: Theoretical analysis and applications," $\mathrm{PhD}$ dissertation, Cambridge university, June 2015.

[34] E. Arikan, "Systematic Polar Coding," IEEE Communications Letters, vol. 15, no. 08, pp. 860-862, August 2011.

[35] S. Korada, R. Urbanke, and N. Macris, "Polar codes for channel and source coding," PhD dissertation, Swiss Federal Institute of Technology at Lausanne (EPFL), June 2009.

[36] K. Niu, K. Chen, J. Lin, and Q. Zhang, "Polar codes: Primary concepts and practical decoding algorithms," IEEE Communications Magazine, vol. 52, no. 07, pp. 192-203, July 2014.

[37] N. Hussami, S. Korada, and R. Urbanke, "Performance of polar codes for channel and source coding," IEEE International Symposium on Information Theory (ISIT), pp. 1488-1492, July 2009.

[38] J. Chen, A. Dholakia, E. Eleftheriou, M. Fossorier, and X. Hu, "Reducedcomplexity decoding of LDPC codes," IEEE Transactions on Communications, vol. 53, no. 08, pp. 1288-1299, August 2005. 
[39] Y. Zhang, A. Liu, X. Pan, Z. Ye, and C. Gong, "A modified belief propagation polar decoder," IEEE Communications Letters, vol. 18, no. 07, July 2014.

[40] J. Zhang, M. Fossorier, and D. Gu, "Two-dimensional correction for min-sum decoding of irregular LDPC codes," IEEE Communications Letters, vol. 10, no. 03, March 2006.

[41] T. Richardson and R. Urbanke, "The capacity of low-density parity check codes," IEEE Transactions on Information Theory, vol. 47, no. 02, pp. 599-618, February 2001.

[42] S. Chung, T. Richardson, and R. Urbanke, "Analysis of sum-product decoding of low-density parity-check codes using a Gaussian approximation," IEEE Transactions on Information Theory, vol. 47, no. 02, pp. 657-670, February 2001.

[43] J. Chen and M. Fossorier, "Density evolution for two improved BP-based decoding algorithms of LDPC codes," IEEE Communications Letters, vol. 06, no. 05, May 2002.

[44] J. Hagenauer, E. Offer, and L. Papke "Iterative decoding of binary block and convolutional codes," IEEE Transactions on Information Theory, vol. 42, pp. 429-445, March 1996.

[45] X. Zhang and P. Siegel, "Quantized iterative message passing decoders with low error floor for LDPC codes," IEEE Transactions on Communications, vol. 62, no. 01, pp. 01-14, December 2013.

[46] S. Papaharalabos and P. Mathiopoulos, "Simplified sum-product algorithm for decoding LDPC codes with optimal performance," IET Electronics Letters, vol. 45, pp. 116-117, Jan. 2009.

[47] T. Clevorn and P. Vary, "Low-complexity belief propagation decoding by approximations with lookup-tables," International ITG Conference on Source and Channel Coding (SCC), pp. 211-215, January 2004.

[48] R. Gallager, "Low-density parity-check codes," MIT Press, Cambridge, 1963.

[49] T. Richardson, M. Shokrollahi, and R. Urbanke, "Design of capacity-approaching irregular low-density parity-check codes," IEEE Transactions on Information Theory, vol. 47, no. 02, pp. 619-637, February 2001.

[50] A. Anastasopoulos, "A comparison between the sum-product and the min-sum iterative detection algorithms based on density evolution," IEEE Global Telecommunications Conference (GLOBECOM), vol. 02, pp. 1021-1025 , November 2001.

[51] R. Mitchell, "Permanence of the log-normal distribution," Journal of the optical Society of America (OSA), vol. 58, no. 09, September 1968.

[52] N. Beaulieu and Q. Xie, "An optimal lognormal approximation to lognormal sum distributions," IEEE Transactions on Vehicular Technology, vol. 53, no. 02, pp. 479-489, March 2004. 
[53] N. Beaulieu, A. Abu-Dayya, and P. McLane, "Estimating the distribution of independent lognormal random variables," IEEE Transactions on Communications, vol. 43, no. 12, pp. 2869-2873, December 1995.

[54] H. Si, O. Koyluoglu, and S. Vishwanath, "Polar coding for fading channels: Binary and exponential channel cases," IEEE Transactions on Communications, vol. 62, no. 08, pp. 2638-2650, August 2009.

[55] À. Bravo-Santos, "Polar Codes for the Rayleigh fading channel," IEEE Communications Letters, vol. 17, no. 12, pp. 2352-2355, November 2013.

[56] M. Dijk, A. Janssen, and A. Koppelaar, "Correcting systematic mismatches in computed log-likelihood ratios," European Transactions on Telecommunications, vol. 14, no. 03, pp. 227-224, January 2003.

[57] G. Lechner and J. Sayir, "Improved sum-min decoding for irregular LDPC codes," Turbo Codes \& Related Topics, International ITG-Conference on Source and Channel Coding (TURBOCODING), pp. 01-06, April 2006.

[58] Leszek Szczecinski, "Correction of mismatched L-values in BICM receivers," IEEE Transactions on Communications, vol. 60, no. 11, pp. 2270-2276, November 2012.

[59] J. Hou, P. Siegel, and L. Milstein, "Performance analysis and code optimization of low density parity-check codes on Rayleigh fading channels," IEEE Journal on Selected Areas in Communications, vol. 19, no. 05, pp. 924-934, May 2001.

[60] L. Papke, P. Robertson, and E. Villebrun, "Improved decoding with the SOVA in a parallel concatenated (Turbo-code) scheme," IEEE Journal on Selected Areas in Communications, vol. 01, pp. 102-106, June 1996.

[61] G. Lechner, "Efficient decoding techniques for LDPC codes," PhD dissertation, Vienna University of Technology, Austria, July 2007.

[62] T. Nguyen and L. Lampe, "Bit-interleaved coded modulation with mismatched decoding metrics," IEEE International Conference on Communications (ICC), vol. 59, no. 02, pp. 437-447, February 2011.

[63] R. Yazdani and M. Ardakani, "Linear LLR approximation for iterative decoding on wireless channels," IEEE Transactions on Communications, vol. 57, no. 11, pp. 3278-3287, November 2009.

[64] M. Tuchler, "Design of serially concatenated systems depending on the block length," IEEE Transactions on Communications, vol. 52, no. 02, pp. 209-218, February 2004.

[65] U. Dasgupta and C. Georghiades, "Turbo decoding of quantized data," IEEE Transactions on Communications, vol. 50, no. 01, pp. 56-64, January 2002.

[66] Z. Shi and K. Niu, "On uniform quantization for successive cancellation decoder of polar codes," IEEE International Symposium on Personal, Indoor, and Mobile Radio Communication (PIMRC), pp. 545-549, September 2014. 
[67] Q. Xie, K. Peng, J. Song, C. Pan, and Z. Yang, "Soft-quantized demodulation for BPSK over discrete-time memoryless fading channel," IEEE International Conference on Communication Systems (ICCS), pp. 855-859, November 2008.

[68] R. Hu, J. Li (Tiffany), and E. Kurtas, "Quantization and quantization sensitivity of soft-output product codes for fast-speed applications," IEEE Sarnoff Symposium on Advances in Wired and Wireless Communications, pp. 175-178, April 2004.

[69] D. Oh and K. K. Parhi, "Low complexity implementations of sum-product algorithm for decoding low-density parity-check codes," IEEE Workshop on Signal Processing Systems Design and Implementation, pp. 262-267, October 2006.

[70] G. Foschini and M. Gans, "On limits of wireless communications in a fading environment when using multiple antennas," Wireless Personal Communications, vol. 55, no. 03, pp. 311-335, March 1998.

[71] A. Goldsmith and P. Varaiya, "Capacity of fading channels with channel side information," IEEE Transactions on Information Theory, vol. 43, no. 06, pp. 1986-1992, November 1997.

[72] S. Sandhu and A. J. Paulraj, "Space-time block codes: Capacity perspective," IEEE Communications Letters, vol. 04, no. 12, pp. 384-386, December 2000.

[73] M. Torabi, M. Soleymani, and S. Aissa, "On the performance of MIMO-OFDM systems with imperfect channel information," IEEE International Conference on Wireless Networks, Communications and Mobile Computing, vol. 01, pp. 600-605, December 2005.

[74] Y. Fang, G. Han, P. Chen, Y. Guan, and G. Bi, "Asymptotic performance analysis of protograph LDPC-coded STBC systems in fading channels," IEEE International Symposium on Personal, Indoor, and Mobile Radio Communication (PIMRC), pp. 132-137, December 2015.

[75] X. Wang, Z. Zhang, and L. Zhang, "On the polar codes for MIMO," IEEE International Conference on Wireless Communications and Signal Processing ( WCSP), pp. 01-05, December 2013.

[76] J. Yang, C. Zhang, W. Song, S. Xu, and X. You, "Joint detection and decoding for MIMO systems with polar codes," IEEE International Symposium on Circuits and Systems (ISCAS), pp. 161-164, August 2016.

[77] B. Feng, J. Jiao, S. Wang, S. Wu, and Q. Zhang, "Construction of polar codes concatenated to space-time block coding in MIMO system," IEEE Vehicular Technology Conference (VTC-Fall), pp. 01-05, September 2016.

[78] I. Telatar, "Capacity of multi-antenna Gaussian channels," European Transactions on Telecommunications, vol. 10, no. 06, pp. 585-595, November 1999.

[79] T. Chan, S. Hranilovic, and F. Kschischang, "Capacity-achieving probability measure for conditionally Gaussian channels with bounded inputs," IEEE Transactions on Information Theory, vol. 51, no. 06, pp. 2073-2088, June 2005. 
[80] J. Bellorado, S. Ghassemzadeh, and A. Kavčić, "Approaching the capacity of the MIMO Rayleigh flat-fading channel with QAM constellations, independent across antennas and dimensions," IEEE Transactions on Communications, vol. 05, no. 06, pp. 1322-1332, June 2006.

[81] D. Le, H. Vu, N. Tran, H. Dinh, and T. Karacolak, "Capacity-achieving signals of non-coherent Rayleigh fading channels with additive Gaussian mixture noise," IEEE International Conference on Communications, Management and Telecommunications (ComManTel), pp. 229-234, December 2015.

[82] S. Shamai (Shitz) and I. David, "The capacity of average and peak-power-limited quadrature Gaussian channels," IEEE Transactions on Information Theory, vol. 41, no. 04, pp. 1060-1071, July 1995.

[83] I. Faycal and S. Shamai (Shitz), "The capacity of discrete-time memoryless rayleigh-fading channels," IEEE Transactions on Information Theory, vol. 47, no. 04, pp. 1290-1301, May 2001.

[84] J. Smith, "The information capacity of peak and average power constrained scalar Gaussian channels," Information and Control, vol. 18, no.03, pp. 203-219, April 1971.

[85] S. Dragomir, "Operator Inequalities of the Jensen, Čebyšev and Grüss Type," Springer Briefs in Mathematics, 2012.

[86] R. Ash, "Information Theory," University of Illinois, Illinois, John Wiley \& Sons, 1965.

[87] X. Wang, E. Serpedin, and K. Qaraqe, "A variational approach for assessing the capacity of a memoryless nonlinear MIMO channel," IEEE Communications Letters, vol. 18, no. 08, pp. 1315-1318, August 2014.

[88] J. Fahs and I. Faycal, "Using Hermite bases in studying capacity-achieving distributions over AWGN channels," IEEE Transactions on Information Theory, vol. 58, no. 08, pp. 5302-5322, August 2012.

[89] F. Tosato and P. Bisaglia, "Simplified soft-output demapper for binary interleaved COFDM with application to HIPERLAN/2," IEEE International Conference on Communications (ICC), vol. 02, pp. 664-668, April 2002.

[90] E. Akay and E. Ayanoglu, "Low complexity decoding of bit-interleaved coded modulation for M-ary QAM," IEEE International Conference on Communications (ICC), vol. 02, pp. 664-668, June 2004.

[91] M. Arar, C. D'Amours, and A. Yongacoglu, "Simplified LLRs for the decoding of single parity check turbo product codes transmitted using 16QAM," Hindawi publishing corporation, research letters in communications, vol. 2007, pp. 1-4, 2007.

[92] M. S. Raju, R. Annavajjala, and A. Chockalingam, "BER analysis of QAM on fading channels with transmit diversity," IEEE Transaction on Wireless Communications, vol. 05, no. 03, March 2006. 
[93] A. Papoulis and S. U. Pillai, "Probability, random variables, and stochastic process," Polytechnic University, Fourth edition, 2002.

[94] G. Djordjević, "On some properties of generalized Hermite polynomials," University of Niš, Faculty of Technology, Yugoslavia, March 1994. 


\section{Appendix A}

\section{Low Computational Complexity LLR Metrics for Polar Coded QAM}

The need of soft decision decoders for robust error corecting codes pushes towards using complicated demappers in order to satisfy LLR metrics. The max-log-MAP demapper as an instance requires $2^{m}$ ( $m$ is the number of bits per symbol) comparisons for LLR computations; this complexity goes higher when high order modulations are applied. In this work we are interest on the approximation of Gray-mapping QAM demmapers. In [89], approximated LLR metrics were introduced for single antenna at both OFDM system sides. The authors in [90] show that the minimum distance for each bit LLR is lowered from $M$ points on a complex constellation to $\sqrt{M}$ points on a real line.

Our contribution here is to show that the soft decision bit metrics for the ML decoder can be significantly simplified to a scaled version of the demapper output where the resultant bit metrics need no computation of minimum distances and their comparisons. We show that the proposed low complexity bit metrics are essentially equivalent to the optimal ones when used for SC decoder of polar codes. We also tabulate the realization utilities of the proposed bit metrics compared to the original metrics in terms of multiplications, additions, and comparisons numbers required.

\section{A.1 System Model}

A 16-QAM constellation is shown in Fig. A.1 where $\log _{2} 16=4$ bits, $\left(b_{1}, b_{2}, b_{3}, b_{4}\right)$, are mapped onto a complex symbol $x=x_{I}+j x_{Q}$ then $x$ is sent over Rayleigh flat 


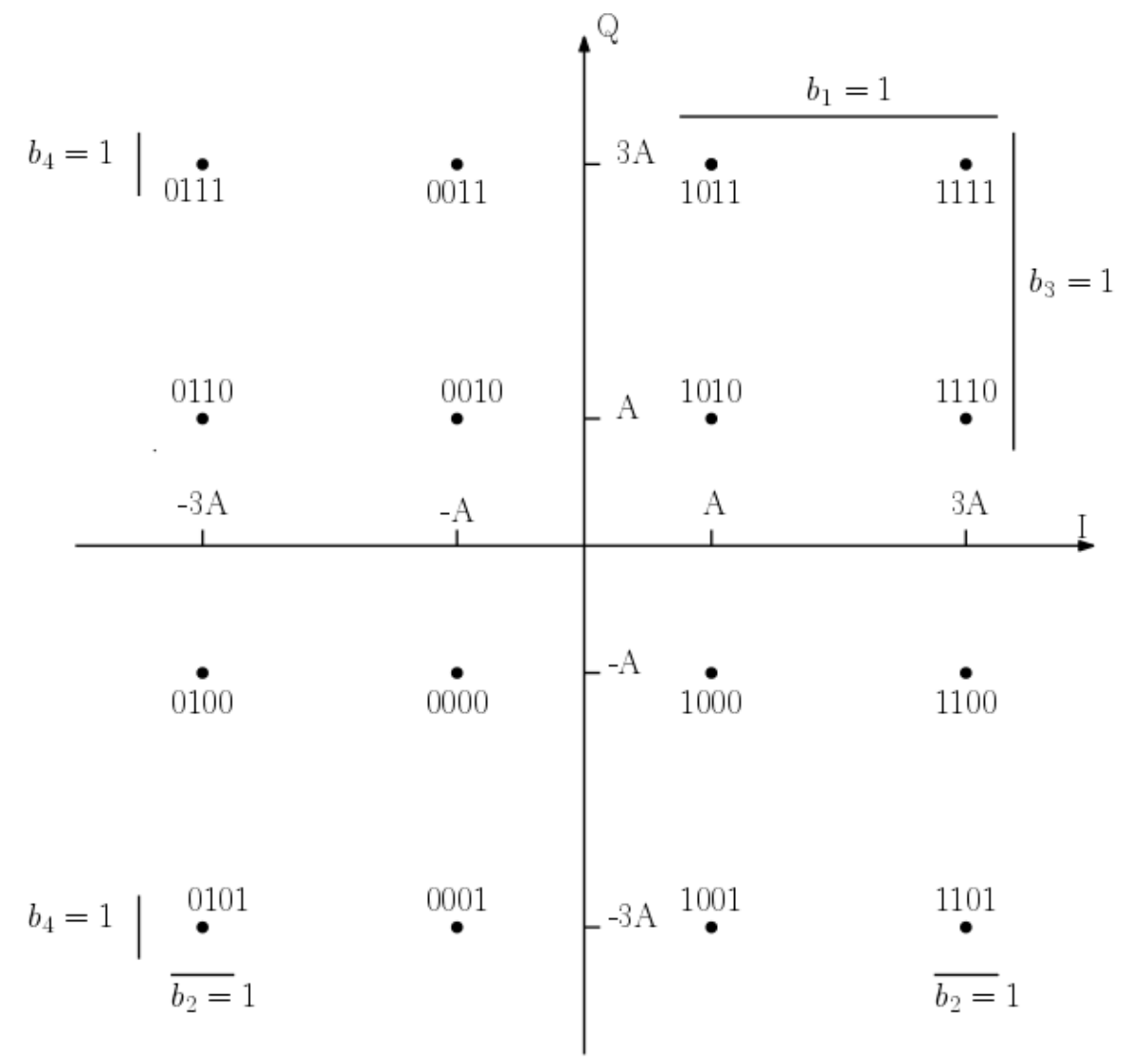

Figure A.1: Detection regions for 16-QAM constellation.

fading channel where the received signal $y$ is

$$
y=h x+n,
$$

$h$ is the complex fading channel coefficient with $\mathrm{E}\left[\|h\|^{2}\right]=1$ and the random variables $\|h\|$ are assumed to be i.i.d exponential distributed. The noise $n=n_{I}+j n_{Q}$ is a complex Gaussian r.v with zero mean and variance $\sigma^{2}$. For bit $b_{i}$ with $i=1,2,3,4$, the LLR of $b_{i}$ is defined as

$$
\lambda_{i}=\ln \frac{\operatorname{Pr}\left\{y \mid h, b_{i}=0\right\}}{\operatorname{Pr}\left\{y \mid h, b_{i}=1\right\}} .
$$

The optimum decision rule is to decide $\hat{b}_{i}=0$ if $\lambda_{i} \geqslant 0$, and 1 otherwise. Define two sets $\mathcal{T}_{i}(0)$ and $\mathcal{T}_{i}(1)$ such that $\mathcal{T}_{i}(k)$ comprises the symbols with $b_{i}=k$ in the 
constellation. Thus we have

$$
\lambda_{i}=\ln \frac{\sum_{x \in \mathcal{T}_{i}(0)} \operatorname{Pr}\{y \mid h, x\}}{\sum_{x \in \mathcal{T}_{i}(1)} \operatorname{Pr}\{y \mid h, x\}} .
$$

Assuming that all the symbols are equally likely and that fading is independent of the transmitted symbols, then according to Baye's rule we get

$$
\lambda_{i}=\ln \frac{\sum_{x \in \mathcal{T}_{i}(0)} f_{y \mid h, x}(y \mid h, x)}{\sum_{x_{i} \in \mathcal{T}_{i}(1)} f_{y \mid h, x}(y \mid h, x)} .
$$

Substituting

$$
f_{y \mid h, x}(y \mid h, x)=\frac{1}{\sqrt{2 \pi \sigma^{2}}} e^{-\frac{\|y-h x\|^{2}}{2 \sigma^{2}}}
$$

gives

$$
\lambda_{i}=\ln \frac{\sum_{x \in \mathcal{T}_{i}(0)} e^{-\frac{\left\|y-h x_{i}\right\|^{2}}{2 \sigma^{2}}}}{\sum_{x \in \mathcal{T}_{i}(1)} e^{-\frac{\|y-h x\|^{2}}{2 \sigma^{2}}}} .
$$

Finally, by using the approximation

$$
\ln \sum_{i} e^{-|x|} \approx-\min _{i}(|x|)
$$

we get

$$
\lambda_{i} \approx \frac{1}{2 \sigma^{2}}\left[\min _{x \in \mathcal{T}_{i}(0)}\|y-h x\|^{2}-\min _{x \in \mathcal{T}_{i}(1)}\|y-h x\|^{2}\right] .
$$

In order to find the bit metric given in the last equation, a Gray mapping is assumed where encoding of the 16-QAM inphase and quadrature parts of the symbols can be done independently. This can be done by separating the $m$ bits into two groups of $\frac{m}{2}$ bits for both the inphase and quadrature components of the symbol [90]. Then for $1 \leqslant i \leqslant \frac{m}{2}$ it is easy to see that for a fixed $i$ the quadrature values of $x \in\left(T_{i}(0)\right.$, 
$\left.T_{i}(1)\right)$ are the same. Hence

$$
\begin{aligned}
& \lambda_{1} \approx \frac{1}{2 \sigma^{2}}\left[\min _{x \in\left\{\frac{-\sigma}{\sqrt{10}}, \frac{-3 \sigma}{\sqrt{10}}\right\}}\|y-h x\|^{2}-\min _{x \in\left\{\frac{\sigma}{\sqrt{10}}, \frac{3 \sigma}{\sqrt{10}}\right\}}\|y-h x\|^{2}\right] \\
& \approx \frac{1}{2 \sigma^{2}} \times \begin{cases}\frac{8 \sigma}{\sqrt{10}}(h y)_{I}-\frac{8\|h\|^{2} \sigma^{2}}{10} & \text { if } \frac{2\|h\|^{2} \sigma}{\sqrt{10}}<(h y)_{I} \\
\frac{4 \sigma}{\sqrt{10}}(h y)_{I} & \text { if }\left|(h y)_{I}\right| \leqslant \frac{2\|h\|^{2} \sigma}{\sqrt{10}} \\
\frac{8 \sigma}{\sqrt{10}}(h y)_{I}+\frac{8\|h\|^{2} \sigma^{2}}{10} & \text { if }(h y)_{I}<\frac{-2\|h\|^{2} \sigma}{\sqrt{10}}\end{cases}
\end{aligned}
$$

where $(h y)_{I}$ is the real part of $(h y)$. The LLR of the second bit, $b_{2}$, is written as

$$
\begin{aligned}
\lambda_{2} & \approx \frac{1}{2 \sigma^{2}}\left[\min _{x \in \frac{-\sigma}{\sqrt{10}}, \frac{\sigma}{\sqrt{10}}}\|y-h x\|^{2}-\min _{x \in \frac{-3 \sigma}{\sqrt{10}}, \frac{3 \sigma}{\sqrt{10}}}\|y-h x\|^{2}\right] \\
& \left.\approx \frac{1}{2 \sigma^{2}}\left[\frac{4 \sigma}{\sqrt{10}}\left|(h y)_{I}\right|-\frac{8\|h\|^{2} \sigma^{2}}{10}\right)\right]
\end{aligned}
$$

$\lambda_{3}$ and $\lambda_{4}$ are similar to $\lambda_{1}$ and $\lambda_{2}$ respectively except that $(h y)_{I}$ is replaced by $(h y)_{Q}$ which is the quadrature phase (imaginary) part of $(h y)$.

\section{A.2 Simplified Bit Metrics}

Maher et al in [91] derived four simple approximate LLR expressions for decoding single parity check turbo product codes (SPC/TPCs). They applied the interpolation method across a specific segment of Fig. A.1 and, by simulation, the proposed method's BER performance for SPC/TPC is virtually identical to the one achieved when using the exact LLRs, which are more cumbersome to compute.

In our work, we take a similar approach and approximate the LLRs, but we do this based on different assumptions, which result in simpler LLR expressions. An LLR linear simplification is realized here per consideration threshold. This consideration threshold is just a value for which the corresponding LLR equals zero, and accordingly there is one or more thresholds for each LLR. For example, $\lambda_{1}$ which is expressed as a ratio of exponentials is

$$
\operatorname{LLR}\left(b_{1}\right)=\ln \frac{e^{-\frac{\left\|y-\frac{h \sigma}{\sqrt{10}}\right\|^{2}}{2 \sigma^{2}}}+e^{-\frac{\left\|y-\frac{3 h \sigma}{\sqrt{10}}\right\|^{2}}{2 \sigma^{2}}}}{e^{-\frac{\left\|y+\frac{h \sigma}{\sqrt{10}}\right\|^{2}}{2 \sigma^{2}}}+e^{-\frac{\left\|y+\frac{3 h \sigma}{\sqrt{10}}\right\|^{2}}{2 \sigma^{2}}}}
$$


It is an increasing function where $y$ and is equal to zero for $y=0$; i.e. has a single decision threshold for $y=0$. Therefore the slope of this LLR function at the decision threshold may be determined by differentiating the LLR function with respect to $y=0$ as follows

$$
\text { Slope }=\left.\frac{\partial \operatorname{LLR}\left(b_{1}\right)}{\partial y}\right|_{y=0}=\frac{4(h)_{I}}{\sqrt{10} \sigma}-\frac{2(h)_{I}}{\sqrt{10} \sigma} \cdot \frac{1-e^{-\frac{4\|h\|^{2}}{10}}}{1+e^{-\frac{4\|h\|^{2}}{10}}} .
$$

For high received SNR, this slope may be approximated as $\frac{2(h)_{I}}{\sqrt{10 \sigma}}$. Then linear approximation of the $\lambda_{1}$ is

$$
\lambda_{1} \approx \frac{2(h y)_{I}}{\sqrt{10} \sigma} .
$$

As shown in Fig. A.2, the last approximation shows a strong degradation with respect to the exact performance. It is impractical to replace such approximation by the formal piece-wise linear one. Instead, we can introduce a suboptimality by averaging over the three linear pieces in (A.9) which could reduce the complexity in terms of complex operations needed for each soft-decision bit metric. Hence

$$
\lambda_{1} \approx \frac{\frac{10}{3}(h y)_{I}}{\sqrt{10} \sigma} .
$$

The factor $\left(\frac{10}{3}\right)$ in (A.14) indicates less degradation than factor (2) in (A.13) as shown also in Fig. A.2.

$\lambda_{3}$ has the same approximated form of $\lambda_{1}$ except that $(h y)_{I}$ is $(h y)_{Q}$, while both $\lambda_{2}$ and $\lambda_{4}$ have no approximations and remain have the same previous forms. 


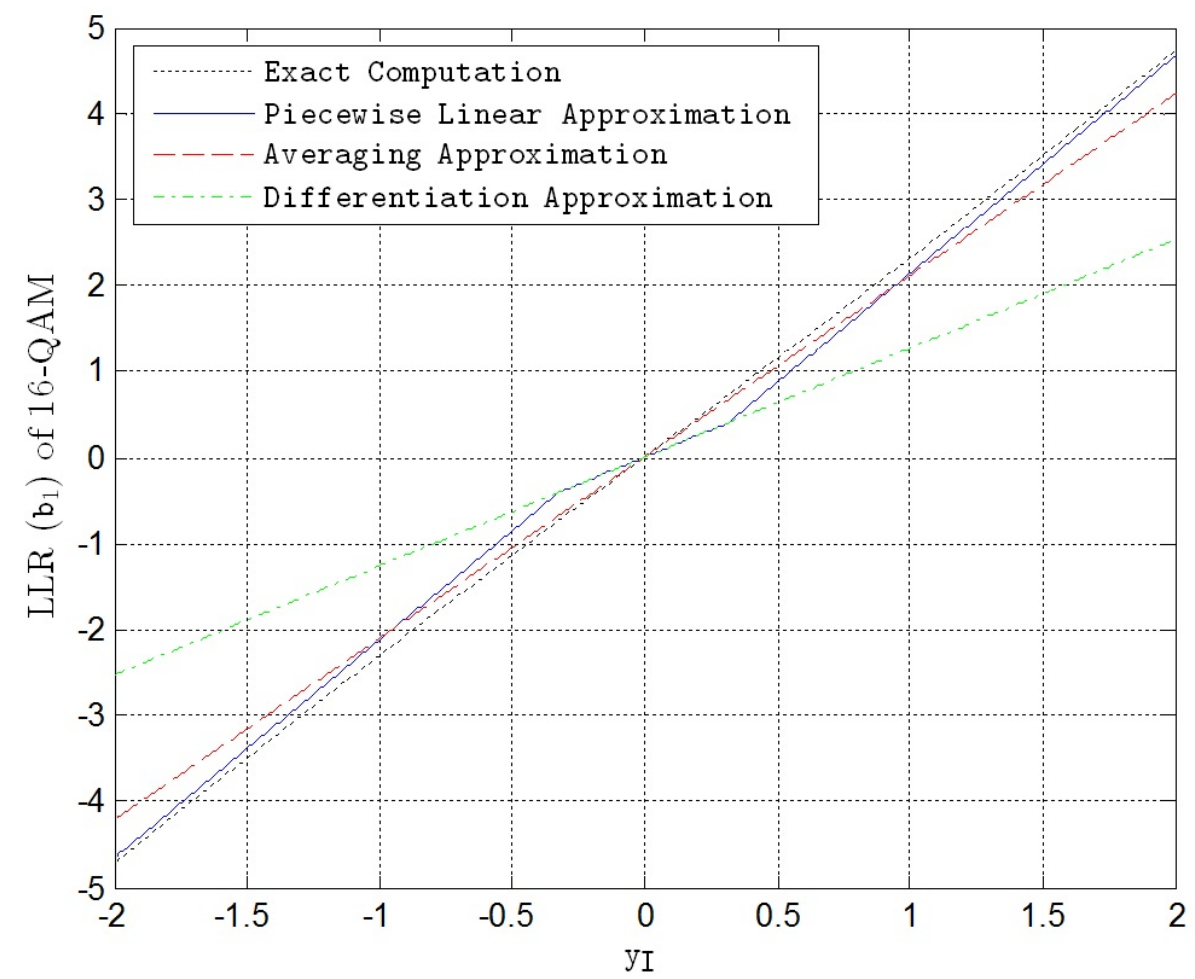

Figure A.2: Exact and approximated (piece-wise linear and by averaging and differentiation) LLR $\left(b_{1}\right)$ for 16-QAM with $|h|=1$ and $\sigma=0.5$.

Similarly for 64-QAM constellation,

$$
\begin{aligned}
& \lambda_{1} \approx \frac{1}{2 \sigma^{2}}\left[\min _{x \in\left\{\frac{-\sigma}{\sqrt{42}}, \frac{-3 \sigma}{\sqrt{42}}, \frac{-5 \sigma}{\sqrt{42}}, \frac{-7 \sigma}{\sqrt{42}}\right\}}\|y-h x\|^{2}-\min _{x \in\left\{\frac{\sigma}{\sqrt{42}}, \frac{3 \sigma}{\sqrt{42}}, \frac{5 \sigma}{\sqrt{42}}, \frac{7 \sigma}{\sqrt{42}}\right\}}\|y-h x\|^{2}\right] \\
& \approx \frac{1}{2 \sigma^{2}} \times \begin{cases}\frac{16 \sigma}{\sqrt{42}}(h y)_{I}-\frac{48\|h\|^{2} \sigma^{2}}{42} & \text { if } \frac{6\|h\|^{2} \sigma}{\sqrt{42}}<(h y)_{I} \\
\frac{12 \sigma}{\sqrt{42}}(h y)_{I}-\frac{24\|h\|^{2} \sigma^{2}}{42} & \text { if } \frac{4\|h\|^{2} \sigma}{\sqrt{42}}<(h y)_{I} \leqslant \frac{6\|h\|^{2} \sigma}{\sqrt{42}} \\
\frac{8 \sigma}{\sqrt{42}}(h y)_{I}-\frac{8\|h\|^{2} \sigma^{2}}{42} & \text { if } \frac{2\|h\|^{2} \sigma}{\sqrt{42}}<(h y)_{I} \leqslant \frac{4\|h\|^{2} \sigma}{\sqrt{42}} \\
\frac{4 \sigma}{\sqrt{42}}(h y)_{I} & \text { if }\left|(h y)_{I}\right| \leqslant \frac{2\|h\|^{2} \sigma}{\sqrt{42}} \\
\frac{8 \sigma}{\sqrt{42}}(h y)_{I}+\frac{8\|h\|^{2} \sigma^{2}}{42} & \text { if } \frac{-4\|h\|^{2} \sigma}{\sqrt{42}} \leqslant(h y)_{I}<\frac{-2\|h\|^{2} \sigma}{\sqrt{42}} \\
\frac{12 \sigma}{\sqrt{42}}(h y)_{I}+\frac{24\|h\|^{2} \sigma^{2}}{42} & \text { if } \frac{-6\|h\|^{2} \sigma}{\sqrt{42}} \leqslant(h y)_{I}<\frac{-4\|h\|^{2} \sigma}{\sqrt{42}} \\
\frac{16 \sigma}{\sqrt{42}}(h y)_{I}+\frac{48\|h\|^{2} \sigma^{2}}{42} & \text { if }(h y)_{I}<\frac{-6\|h\|^{2} \sigma}{\sqrt{42}}\end{cases} \\
& \approx \frac{\frac{38}{7}(h y)_{I}}{\sqrt{42} \sigma}
\end{aligned}
$$




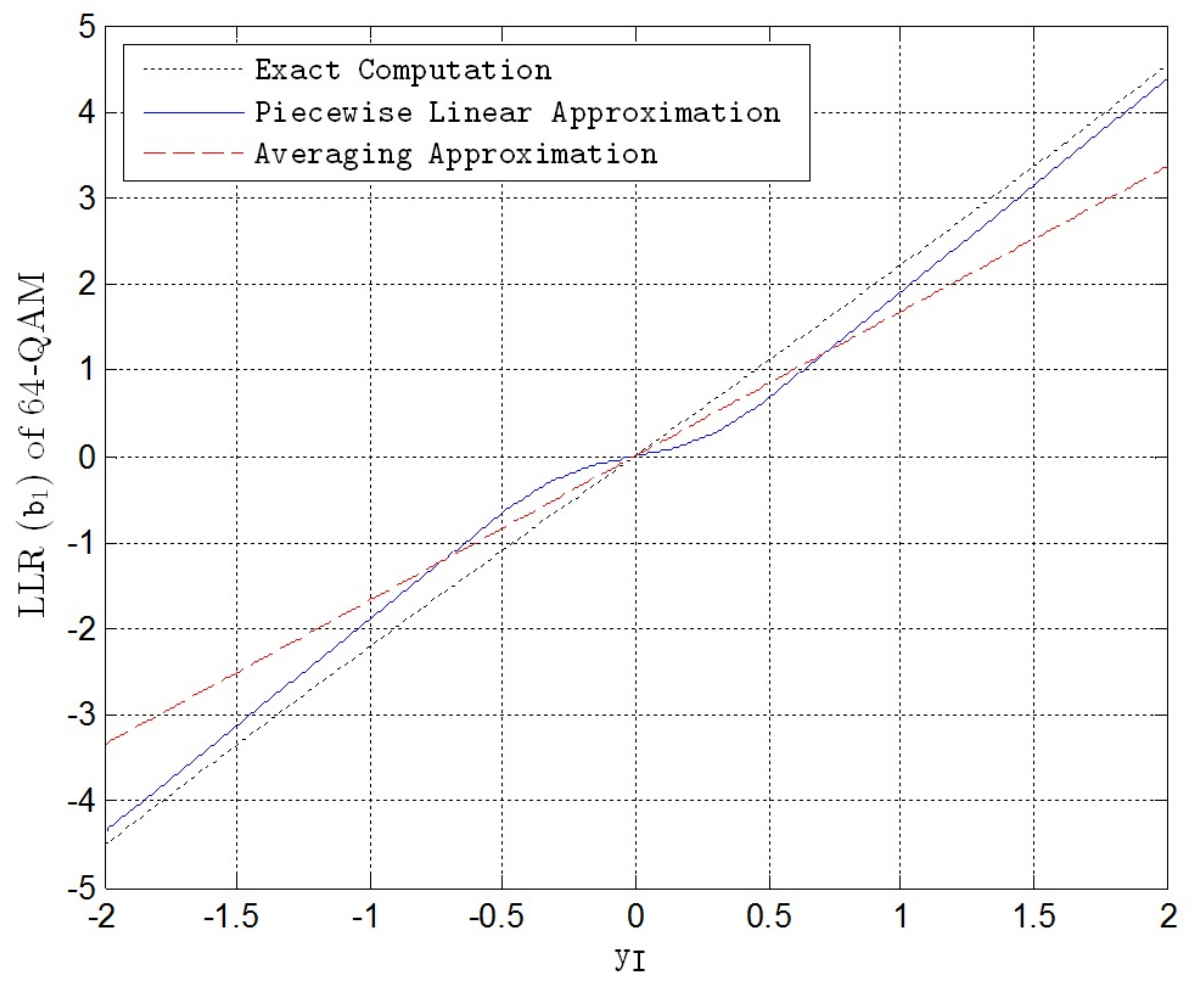

Figure A.3: Exact and approximated (piece-wise linear and by differentiation) $\operatorname{LLR}\left(b_{1}\right)$ for $64-$ QAM with $|h|=1$ and $\sigma=0.5$.

Again we have introduced a slight suboptimality in the last step by averaging over seven possible bit metrics as shown in Fig. A.3. Similarly for the second bit, LLR $\left(b_{2}\right)$ is written as

$$
\begin{aligned}
& \lambda_{2} \approx \frac{1}{2 \sigma^{2}}\left[\min _{x \in\left\{\frac{-\sigma}{\sqrt{42}}, \frac{-3 \sigma}{\sqrt{42}}, \frac{\sigma}{\sqrt{42}}, \frac{3 \sigma}{\sqrt{42}}\right\}}\|y-h x\|^{2}-\min _{x \in\left\{\frac{-5 \sigma}{\sqrt{42}}, \frac{-7 \sigma}{\sqrt{42}}, \frac{5 \sigma}{\sqrt{42}}, \frac{7 \sigma}{\sqrt{42}}\right\}}\|y-h x\|^{2}\right] \\
& \approx \frac{1}{2 \sigma^{2}} \times \begin{cases}\frac{8 \sigma}{\sqrt{42}}\left|(h y)_{I}\right|-\frac{40\|h\|^{2} \sigma^{2}}{42} & \text { if } \frac{6\|h\|^{2} \sigma}{\sqrt{42}}<\left|(h y)_{I}\right| \\
\frac{4 \sigma}{\sqrt{42}}\left|(h y)_{I}\right|-\frac{16\|h\|^{2} \sigma^{2}}{42} & \text { if } \frac{2\|h\|^{2} \sigma}{\sqrt{42}}<\left|(h y)_{I}\right|<\frac{6\|h\|^{2} \sigma}{\sqrt{42}} \\
\frac{8 \sigma}{\sqrt{42}}\left|(h y)_{I}\right|-\frac{24\|h\|^{2} \sigma^{2}}{42} & \text { if }\left|(h y)_{I}\right|<\frac{2\|h\|^{2} \sigma}{\sqrt{42}}\end{cases} \\
& \approx \frac{\frac{10}{3}\left|(h y)_{I}\right|}{\sqrt{42} \sigma}-\frac{\frac{40}{3}|| h \|^{2}}{42}
\end{aligned}
$$




$$
\begin{aligned}
\lambda_{3} & \approx \frac{1}{2 \sigma^{2}}\left[\min _{x \in\left\{\frac{-3 \sigma}{\sqrt{42}}, \frac{-5 \sigma}{\sqrt{42}}, \frac{3 \sigma}{\sqrt{42}}, \frac{5 \sigma}{\sqrt{42}}\right\}}\|y-h x\|^{2}-\min _{x \in\left\{\frac{-\sigma}{\sqrt{42}}, \frac{-7 \sigma}{\sqrt{42}}, \frac{\sigma}{\sqrt{42}}, \frac{7 \sigma}{\sqrt{42}}\right\}}\|y-h x\|^{2}\right] \\
& \approx \frac{1}{2 \sigma^{2}} \times \begin{cases}\frac{2 \sigma}{\sqrt{42}}\left|(h y)_{I}\right|-\frac{24\|h\|^{2} \sigma^{2}}{42} & \text { if } \frac{4\|h\|^{2} \sigma}{\sqrt{42}}<\left|(h y)_{I}\right| \\
\frac{-4 \sigma}{\sqrt{42}}\left|(h y)_{I}\right|+\frac{8\|h\|^{2} \sigma^{2}}{42} & \text { if }\left|(h y)_{I}\right|<\frac{4\|h\|^{2} \sigma}{\sqrt{42}}\end{cases} \\
& \approx\left|\frac{2\left|(h y)_{I}\right|}{\sqrt{42} \sigma}-\frac{\left.8|| h\right|^{2}}{42}\right|-\frac{4\|h\|^{2}}{42} .
\end{aligned}
$$

Finally, $\lambda_{4}, \lambda_{5}$ and $\lambda_{6}$ are similar to $\lambda_{1}, \lambda_{2}$ and $\lambda_{3}$ respectively with $(h y)_{I}$ being replaced by $(h y)_{Q}$.

\section{A.3 LLR in Transmit Diversity}

Consider a space-time block code system with two transmit antennas (Alamouti's scheme) [92]. We are going to analyze the case with two transmit antennas and one receive antenna. Assume that the channel remains constant over two consecutive symbol intervals, then the received signals are

$$
\begin{aligned}
& y_{1}=h_{1} x_{1}-h_{2} x_{2}^{*}+n_{1} \quad \text { and } \\
& y_{2}=h_{2} x_{1}^{*}+h_{1} x_{2}+n_{2},
\end{aligned}
$$

where $h_{1}$ and $h_{2}$ are complex fading coefficients with $\mathrm{E}\left[\left\|h_{1}\right\|^{2}\right]=\mathrm{E}\left[\left\|h_{2}\right\|^{2}\right]=1$, and $n_{1}$ and $n_{2}$ are two complex random variables of zero mean and $\sigma^{2}$ variance. Then with perfect CSI we can calculate

$$
\begin{aligned}
& \tilde{x}_{1}=h_{1}^{*} y_{1}+h_{2} y_{2}^{*}=\left(\left\|h_{1}\right\|^{2}+\left\|h_{2}\right\|^{2}\right) x_{1}+n_{1} h_{1}^{*}+n_{2}^{*} h_{2}, \\
& \tilde{x}_{2}=h_{1}^{*} y_{2}-h_{2} y_{1}^{*}=\left(\left\|h_{1}\right\|^{2}+\left\|h_{2}\right\|^{2}\right) x_{2}+n_{2} h_{1}^{*}-n_{1}^{*} h_{2} .
\end{aligned}
$$

Hence, for 16-QAM,

$$
\begin{aligned}
\operatorname{LLR}_{x_{j}}\left(b_{i}\right) & =\ln \frac{\operatorname{Pr}\left\{\tilde{x}_{j} \mid h_{1}, h_{2}, b_{i}=0\right\}}{\operatorname{Pr}\left\{\tilde{x}_{j} \mid h_{1}, h_{2}, b_{i}=1\right\}} \\
& =\ln \frac{\sum_{x_{j} \in \mathrm{T}_{i}(0)} f_{\tilde{x}_{j} \mid h_{1}, h_{2}, x_{j}}\left(\tilde{x}_{j} \mid h_{1}, h_{2}, x_{j}\right)}{\sum_{x_{j} \in \mathrm{T}_{i}(1)} f_{\tilde{x}_{j} \mid h_{1}, h_{2}, x_{j}}\left(\tilde{x}_{j} \mid h_{1}, h_{2}, x_{j}\right)}
\end{aligned}
$$


Table A.1: LLRs calculations complexity in terms of complex operations. $\mathrm{O}$ is Original and S is Simplified.

\begin{tabular}{ccccccc}
\hline & \multicolumn{2}{c}{ Multiplications } & \multicolumn{2}{c}{ Additions } & \multicolumn{2}{c}{ Comparisons } \\
M-ary & O & S & O & S & O & S \\
\hline 4 & 4 & 1 & 6 & 0 & 2 & 0 \\
16 & 16 & 2 & 24 & 0 & 8 & 0 \\
64 & 64 & 2 & 96 & 0 & 32 & 0 \\
\hline
\end{tabular}

where

$$
f_{\tilde{x}_{j} \mid h_{1}, h_{2}, x_{j}}\left(\tilde{x}_{j} \mid h_{1}, h_{2}, x_{j}\right)=\frac{1}{\sqrt{2 \pi \tilde{\sigma}^{2}}} e^{-\frac{\left\|\tilde{x}_{j}-\left(\left\|h_{1}\right\|^{2}+\left\|h_{2}\right\|^{2}\right) x_{j}\right\|^{2}}{2 \sigma^{2}}}
$$

and

$$
\tilde{\sigma}^{2}=\sigma^{2}\left(\left\|h_{1}\right\|^{2}+\left\|h_{2}\right\|^{2}\right) \quad \text { for } i=1,2,3,4 \text { and } j=1,2 .
$$

Following the same presented analysis,

$$
\begin{gathered}
\operatorname{LLR}_{x_{j}}\left(b_{1}\right) \approx \frac{\frac{10}{3}\left(\tilde{x}_{j}\right)_{I}}{\sqrt{10} \tilde{\sigma}} \quad \text { and } \\
\left.\operatorname{LLR}_{x_{j}}\left(b_{2}\right)=\frac{1}{2 \tilde{\sigma}^{2}}\left[\frac{4 \tilde{\sigma}}{\sqrt{10}}\left|\left(\tilde{x}_{j}\right)_{I}\right|-\frac{8\|h\|^{2} \tilde{\sigma}^{2}}{10}\right)\right]
\end{gathered}
$$

$\lambda_{3}$ and $\lambda_{4}$ have the same formulation of $\lambda_{1}$ and $\lambda_{2}$ respectively except that $\left(\tilde{x}_{j}\right)_{I}$ is replaced by $\left(\tilde{x}_{j}\right)_{Q}$ which is the quadrature phase (imaginary) part of $\left(\tilde{x}_{j}\right)$.

The complexity analysis of these bit metrics in terms of complex operations (complex multiplications, complex additions) is tabulated as in TABLE A.1.

\section{A.4 Numerical Results}

We consider the original ML bit metrics and the simplified metrics due averaging. We compare the performance of BER evaluated using the approximate LLRs presented 


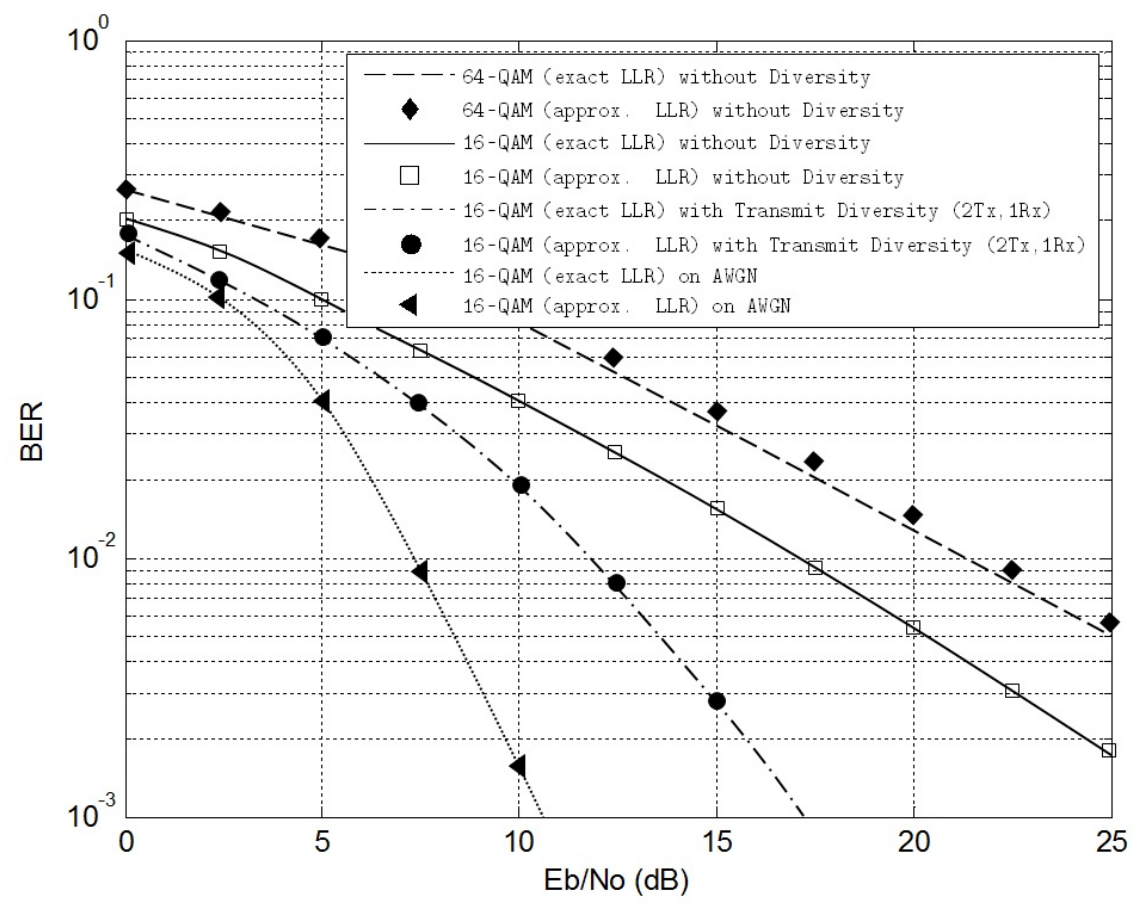

Figure A.4: BER performance of uncoded 16/64-QAM with/without approximation and with/without transmit diversity.

in the above versus with that observed by simulation using the LLRs without approximation, for 16-QAM and 64-QAM schemes on AWGN and flat Rayleigh fading channals with perfect CSI at the receiver. Fig. A.4 shows that the performance of the simplified bit metrics for 16-QAM is almost the same as the exact BER, indicating that the approximation to the LLRs results in insignificant difference between the approximated BER and the true one. While there is a slight degradation in the performance for 64-QAM case. It is imputed to four approximated bit metrics compared to two approximated bit metrics for 16-QAM. It also can be seen that when transmit diversity using Alamouti's scheme is employed, the approximated BER performance improves as expected.

We employed the LLRs as soft inputs to the polar decoder in a rate $\frac{1}{2}$ SC polar coded 16-QAM scheme in both AWGN and Rayleigh fading channals without and with LLRs simplification. Fig. A.5 shows BER performances of SC polar coded 16-QAM system using exact as well as simplified LLRs as soft inputs to the decoder. They are almost the same. 


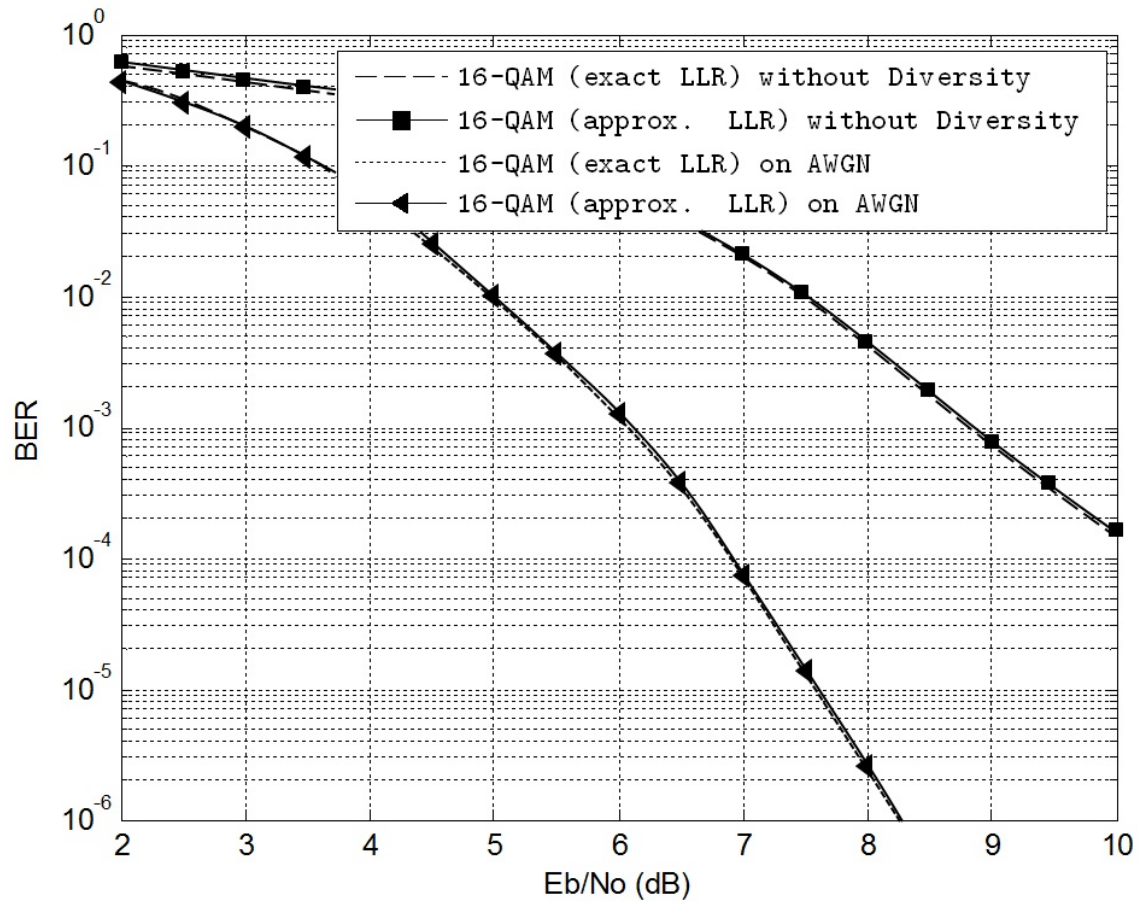

Figure A.5: BER performance of SC polar coded 16-QAM with/without approximation. $R=0.5$ and $N=1024$.

\section{A.5 Summary}

We analyzed the BER performance of 16/64-QAM schemes in AWGN and Rayleigh fading channels without and with transmit diversity. This analysis extends to the use of SC polar coded 16-QAM scheme, and both of BER performances are simulated with exact and approximated LLRs calculations. It is showed that for 16-QAM system the new simplified metrics have the same performance as the original ones while the complexity of a polar decoder can be significantly lowered without compromising the performance. The 64-QAM scheme exhibited a noticeable degradation. 


\section{Appendix B}

\section{Proofs and Derivation}

\section{B.1 Proof of Equation (3.36)}

For simplicity, suppose $x \in\{ \pm 1\}$ with equal a prior probability is transmitted over

an AWGN channel with double-sided noise power spectral density $\sigma^{2}=\frac{N_{o}}{2}$. The received sample, $y=x+n$, has a Gaussian likelihood function given $x$ transmitted of

$$
f_{Y}(y \mid x)=\frac{1}{\sqrt{2 \pi \sigma^{2}}} e^{-\frac{(y-x)^{2}}{2 \sigma^{2}}}
$$

and the PDF of $y$ is

$$
f_{Y}(y)=\frac{1}{2} f_{Y}(y \mid x=-1)+\frac{1}{2} f_{Y}(y \mid x=+1)
$$

Then the calculated LLR of the channel output is

$$
\lambda=\frac{2 y}{\sigma^{2}} .
$$

The CDF of $\lambda$ is

$$
\begin{aligned}
F_{\lambda}(\lambda) & =\operatorname{Pr}\left\{\frac{2 Y}{\sigma^{2}} \leqslant \lambda\right\} \\
& =\operatorname{Pr}\left\{Y \leqslant \frac{\sigma^{2}}{2} \lambda\right\} \\
& =F_{Y}\left(\frac{\sigma^{2}}{2} \lambda\right)
\end{aligned}
$$


But

$$
\begin{aligned}
F_{Y}(y) & =\operatorname{Pr}\{Y \leqslant y\} \\
& =\int_{-\infty}^{y} f_{Y}(\alpha) \mathrm{d} \alpha \\
& =\int_{-\infty}^{y}\left[\frac{1}{2} f_{Y \mid X}(\alpha \mid X=-1)+\frac{1}{2} f_{Y \mid X}(\alpha \mid X=+1)\right] \mathrm{d} \alpha \\
& =\frac{1}{2} F_{Y \mid X}(y \mid X=-1)+\frac{1}{2} F_{Y \mid X}(y \mid X=+1),
\end{aligned}
$$

where

$$
\begin{aligned}
F_{Y \mid X}(y \mid X=x) & =\int_{-\infty}^{y} f_{Y \mid X}(\alpha \mid X=x) \mathrm{d} \alpha \\
& =1-\int_{y}^{\infty} f_{Y \mid X}(\alpha \mid X=x) \mathrm{d} \alpha \\
& =1-\int_{y}^{\infty} \frac{1}{\sqrt{2 \pi \sigma^{2}}} e^{-\frac{(\alpha-x)^{2}}{2 \sigma^{2}}} \mathrm{~d} \alpha \\
& =1-\int_{\frac{y-x}{\sigma}}^{\infty} \frac{1}{\sqrt{2 \pi \sigma^{2}}} e^{-\frac{u^{2}}{2}} \mathrm{~d} u \\
& =1-\mathrm{Q}\left(\frac{y-x}{\sigma}\right) .
\end{aligned}
$$

By substituting (B.6) in (B.5) we get

$$
\begin{aligned}
F_{Y}(y) & =\frac{1}{2}-\frac{1}{2} \mathrm{Q}\left(\frac{y+1}{\sigma}\right)+\frac{1}{2}-\frac{1}{2} \mathrm{Q}\left(\frac{y-1}{\sigma}\right) \\
& =1-\frac{1}{2} \mathrm{Q}\left(\frac{y+1}{\sigma}\right)-\frac{1}{2} \mathrm{Q}\left(\frac{y-1}{\sigma}\right)
\end{aligned}
$$

And then substituting the last result in (B.4) gives

$$
\begin{aligned}
F_{\lambda}(\lambda) & =F_{Y}\left(\frac{\sigma^{2}}{2} \lambda\right) \\
& =1-\frac{1}{2} \mathrm{Q}\left(\frac{\frac{\sigma^{2}}{2} \lambda+1}{\sigma}\right)-\frac{1}{2} \mathrm{Q}\left(\frac{\frac{\sigma^{2}}{2} \lambda-1}{\sigma}\right) \\
& =1-\frac{1}{2} \mathrm{Q}\left(\frac{\lambda+\frac{2}{\sigma^{2}}}{\frac{2}{\sigma}}\right)-\frac{1}{2} \mathrm{Q}\left(\frac{\lambda-\frac{2}{\sigma^{2}}}{\frac{2}{\sigma}}\right) .
\end{aligned}
$$


Now, let $W=|\lambda|$, we have

$$
\begin{aligned}
F_{W}(w) & =\operatorname{Pr}\{W \leqslant w\} \\
& =\operatorname{Pr}\{|\lambda| \leqslant w\} \\
& =\operatorname{Pr}\{-w \leqslant \lambda \leqslant w\} \\
& =F_{\lambda}(w)-F_{\lambda}(-w) \\
& =F_{\lambda}(w)-\left[1-F_{\lambda}(w)\right] \\
& =2 F_{\lambda}(w)-1 .
\end{aligned}
$$

Finally, substituting (B.8) in (B.9) gives

$$
\begin{aligned}
F_{W}(w) & =2-\mathrm{Q}\left(\frac{w+\frac{2}{\sigma^{2}}}{\frac{2}{\sigma}}\right)-\mathrm{Q}\left(\frac{w-\frac{2}{\sigma^{2}}}{\frac{2}{\sigma}}\right)-1 \\
& =1-\mathrm{Q}\left(\frac{w+\frac{2}{\sigma^{2}}}{\frac{2}{\sigma}}\right)-\mathrm{Q}\left(\frac{w-\frac{2}{\sigma^{2}}}{\frac{2}{\sigma}}\right),
\end{aligned}
$$

which completes the proof.

\section{B.2 Derivation of Equation (3.51)}

In order to calculate $\sigma_{\ell}^{2}$ we need to find the expectation, $\mathrm{E}[$.$] , of \left|\tilde{\lambda}_{2}\right|^{2}$ where $\sigma_{\ell}^{2}=\mathrm{E}\left[\left|\tilde{\lambda}_{2}\right|^{2}\right]-\left(\mathrm{E}\left[\left|\tilde{\lambda}_{2}\right|\right]\right)^{2}$, and $\mathrm{E}\left[\left|\tilde{\lambda}_{2}\right|\right]$ is calculated as in Subsection (3.2.1); $\tilde{\lambda}_{2}$ is the corrected LLR.

Now, from (3.15) and (3.16) we have

$$
\begin{aligned}
\left|\tilde{\lambda}_{2}\right|= & \mid \operatorname{sgn}\left(\lambda_{0}\right) \operatorname{sgn}\left(\lambda_{1}\right) \cdot \min \left(\left|\lambda_{0}\right|,\left|\lambda_{1}\right|\right)+\max \left(\ln 2-0.5\left|\lambda_{0}+\lambda_{1}\right|, 0\right) \\
& \quad-\max \left(\ln 2-0.5\left|\lambda_{0}-\lambda_{1}\right|, 0\right) \mid
\end{aligned}
$$


Then

$$
\begin{aligned}
\left|\tilde{\lambda}_{2}\right|^{2}= & \mid \operatorname{sgn}\left(\lambda_{0}\right) \operatorname{sgn}\left(\lambda_{1}\right) \cdot \min \left(\left|\lambda_{0}\right|,\left|\lambda_{1}\right|\right)+\max \left(\ln 2-0.5\left|\lambda_{0}+\lambda_{1}\right|, 0\right) \\
& -\left.\max \left(\ln 2-0.5\left|\lambda_{0}-\lambda_{1}\right|, 0\right)\right|^{2} \\
= & {\left[\min \left(\left|\lambda_{0}\right|,\left|\lambda_{1}\right|\right)\right]^{2} } \\
& +2 \min \left(\left|\lambda_{0}\right|,\left|\lambda_{1}\right|\right) \cdot\left[\max \left(\ln 2-0.5\left|\lambda_{0}+\lambda_{1}\right|, 0\right)-\max \left(\ln 2-0.5\left|\lambda_{0}-\lambda_{1}\right|, 0\right)\right] \\
& +\left[\max \left(\ln 2-0.5\left|\lambda_{0}+\lambda_{1}\right|, 0\right)-\max \left(\ln 2-0.5\left|\lambda_{0}-\lambda_{1}\right|, 0\right)\right]^{2} \\
= & \min \left(\left|\lambda_{0}\right|,\left|\lambda_{1}\right|\right) \cdot \min \left(\left|\lambda_{0}\right|,\left|\lambda_{1}\right|\right) \\
& +2 \min \left(\left|\lambda_{0}\right|,\left|\lambda_{1}\right|\right) \cdot\left[\ln 2-0.5\left|\lambda_{0}+\lambda_{1}\right|-\ln 2+0.5\left|\lambda_{0}-\lambda_{1}\right|\right] \\
& +\left[\ln 2-0.5\left|\lambda_{0}+\lambda_{1}\right|-\ln 2+0.5\left|\lambda_{0}-\lambda_{1}\right|\right]^{2} \\
= & \min \left(\left|\lambda_{0}\right|,\left|\lambda_{1}\right|\right) \cdot \min \left(\left|\lambda_{0}\right|,\left|\lambda_{1}\right|\right)+\min \left(\left|\lambda_{0}\right|,\left|\lambda_{1}\right|\right) \cdot\left[\left|\lambda_{0}-\lambda_{1}\right|-\left|\lambda_{0}+\lambda_{1}\right|\right] \\
& +0.25\left[\left|\lambda_{0}-\lambda_{1}\right|-\left|\lambda_{0}+\lambda_{1}\right|\right]^{2} .
\end{aligned}
$$

Let

$$
\begin{gathered}
\lambda_{\alpha}=\min \left(\left|\lambda_{0}\right|,\left|\lambda_{1}\right|\right) \cdot \min \left(\left|\lambda_{0}\right|,\left|\lambda_{1}\right|\right), \\
\lambda_{\beta}=\min \left(\left|\lambda_{0}\right|,\left|\lambda_{1}\right|\right) \cdot\left[\left|\lambda_{0}-\lambda_{1}\right|-\left|\lambda_{0}+\lambda_{1}\right|\right],
\end{gathered}
$$

and

$$
\lambda_{\gamma}=0.25\left[\left|\lambda_{0}-\lambda_{1}\right|-\left|\lambda_{0}+\lambda_{1}\right|\right]^{2} .
$$

The expectation of $\left|\tilde{\lambda}_{2}\right|^{2}$ is calculated as the following

$$
\mathrm{E}\left[\left|\tilde{\lambda}_{2}\right|^{2}\right]=\int_{0}^{\infty} R_{\left|\tilde{\lambda}_{2}\right|^{2}}(\ell) \mathrm{d} \ell-\int_{-\infty}^{0} F_{\left|\tilde{\lambda}_{2}\right|^{2}}(\ell) \mathrm{d} \ell,
$$

where $F_{\left|\tilde{\lambda}_{2}\right|^{2}}(\ell)$ is the empirical distribution of $(\ell)$ [93] and

$$
R_{\left|\tilde{\lambda}_{2}\right|^{2}}(\ell)=1-F_{\left|\tilde{\lambda}_{2}\right|^{2}}(\ell)=\operatorname{Pr}\left\{\left|\tilde{\lambda}_{2}\right|^{2}>\ell\right\} .
$$


Obviously, to find $F_{\left|\tilde{\lambda}_{2}\right|^{2}}(\ell)$ we need to solve for the empirical CDFs of $\lambda_{\alpha}\left(F_{\lambda_{\alpha}}(\ell)\right)$, $\lambda_{\beta}\left(F_{\lambda_{\beta}}(\ell)\right)$, and $\lambda_{\gamma}\left(F_{\lambda_{\gamma}}(\ell)\right)$. Thus, $\lambda_{\alpha}$ in (B.12) above is simplified as

$$
\begin{aligned}
\lambda_{\alpha} & =\min \left(\left|\lambda_{0}\right|,\left|\lambda_{1}\right|\right) \cdot \min \left(\left|\lambda_{0}\right|,\left|\lambda_{1}\right|\right) \\
& = \begin{cases}\lambda_{0}^{2} & \text { if } \lambda_{0}<\lambda_{1} \\
\lambda_{1}^{2} & \text { otherwise }\end{cases}
\end{aligned}
$$

and hence

$$
\begin{aligned}
F_{\lambda_{\alpha}}(\ell) & =\operatorname{Pr}\left\{\lambda_{\alpha} \leqslant \ell\right\} \\
& =\operatorname{Pr}\left\{\lambda_{0}^{2} \leqslant \ell, \lambda_{0}<\lambda_{1}\right\}+\operatorname{Pr}\left\{\lambda_{1}^{2} \leqslant \ell, \lambda_{0} \geqslant \lambda_{1}\right\} \\
& =\operatorname{Pr}\left\{-\sqrt{\ell} \leqslant \lambda_{0} \leqslant \sqrt{\ell}, \lambda_{0}<\lambda_{1}\right\}+\operatorname{Pr}\left\{-\sqrt{\ell} \leqslant \lambda_{1} \leqslant \sqrt{\ell}, \lambda_{0} \geqslant \lambda_{1}\right\} .
\end{aligned}
$$

The shaded areas in Fig. B.1 show the regions satisfying the corresponding inequalities in each term of (B.18). Accordingly

$$
\begin{aligned}
F_{\lambda_{\alpha}}(\ell)= & 1-\operatorname{Pr}\left\{\lambda_{0}>\sqrt{\ell}, \lambda_{1}>\sqrt{\ell}\right\}-\operatorname{Pr}\left\{\lambda_{0} \leqslant-\sqrt{\ell}\right\}-\operatorname{Pr}\left\{\lambda_{1} \leqslant-\sqrt{\ell}\right\} \\
& +\operatorname{Pr}\left\{\lambda_{0} \leqslant-\sqrt{\ell}, \lambda_{1} \leqslant-\sqrt{\ell}\right\} \\
= & F_{\lambda_{0}}(\sqrt{\ell})+F_{\lambda_{1}}(\sqrt{\ell})-F_{\lambda_{0}}(\sqrt{\ell}) F_{\lambda_{1}}(\sqrt{\ell})-F_{\lambda_{0}}(-\sqrt{\ell})-F_{\lambda_{1}}(-\sqrt{\ell}) \\
& +F_{\lambda_{0}}(-\sqrt{\ell}) F_{\lambda_{1}}(-\sqrt{\ell})
\end{aligned}
$$

It is assumed in Subsection (3.2.1), equation (3.28) and beyond, that the densities of $\lambda_{0}$ and $\lambda_{1}$ are the same. Hence

$$
F_{\lambda_{\alpha}}(\ell)=2 F_{\lambda_{0}}(\sqrt{\ell})-F_{\lambda_{0}}^{2}(\sqrt{\ell})-2 F_{\lambda_{0}}(-\sqrt{\ell})+F_{\lambda_{0}}^{2}(-\sqrt{\ell}) .
$$




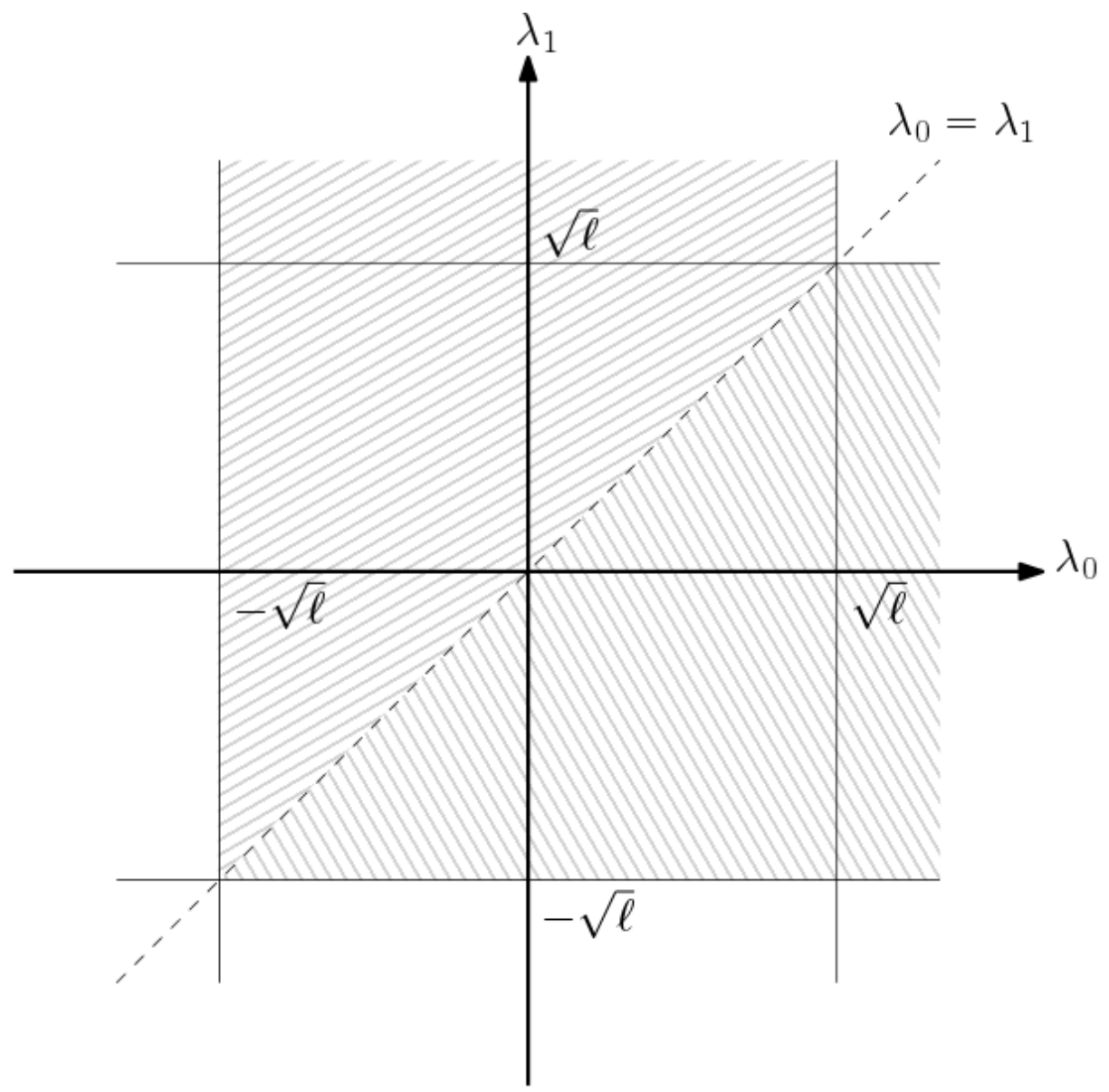

Figure B.1: $F_{\lambda_{\alpha}}$.

Similarly, to solve for $F_{\lambda_{\beta}}(\ell),($ B.13) can be simplified as

$$
\begin{aligned}
\lambda_{\beta} & =\min \left(\left|\lambda_{0}\right|,\left|\lambda_{1}\right|\right) \cdot\left[\left|\lambda_{0}-\lambda_{1}\right|-\left|\lambda_{0}+\lambda_{1}\right|\right] \\
& = \begin{cases}\lambda_{0}\left(-\lambda_{0}+\lambda_{1}-\lambda_{0}-\lambda_{1}\right) & \text { if } \lambda_{0}<\lambda_{1} \\
\lambda_{1}\left(\lambda_{0}-\lambda_{1}-\lambda_{0}-\lambda_{1}\right) & \text { if } \lambda_{1}<\lambda_{0} \\
-\lambda_{0}\left(-\lambda_{0}+\lambda_{1}+\lambda_{0}+\lambda_{1}\right) & \text { if } \lambda_{0} \geqslant \lambda_{1} \\
-\lambda_{1}\left(\lambda_{0}-\lambda_{1}+\lambda_{0}+\lambda_{1}\right) & \text { if } \lambda_{1} \geqslant \lambda_{0}\end{cases} \\
& = \begin{cases}-2 \lambda_{0}^{2} & \text { if } \lambda_{0}<\lambda_{1} \\
-2 \lambda_{1}^{2} & \text { if } \lambda_{1}<\lambda_{0} \\
-2 \lambda_{0} \lambda_{1} & \text { if } \lambda_{0} \geqslant \lambda_{1} \\
-2 \lambda_{0} \lambda_{1} & \text { if } \lambda_{1} \geqslant \lambda_{0}\end{cases}
\end{aligned}
$$




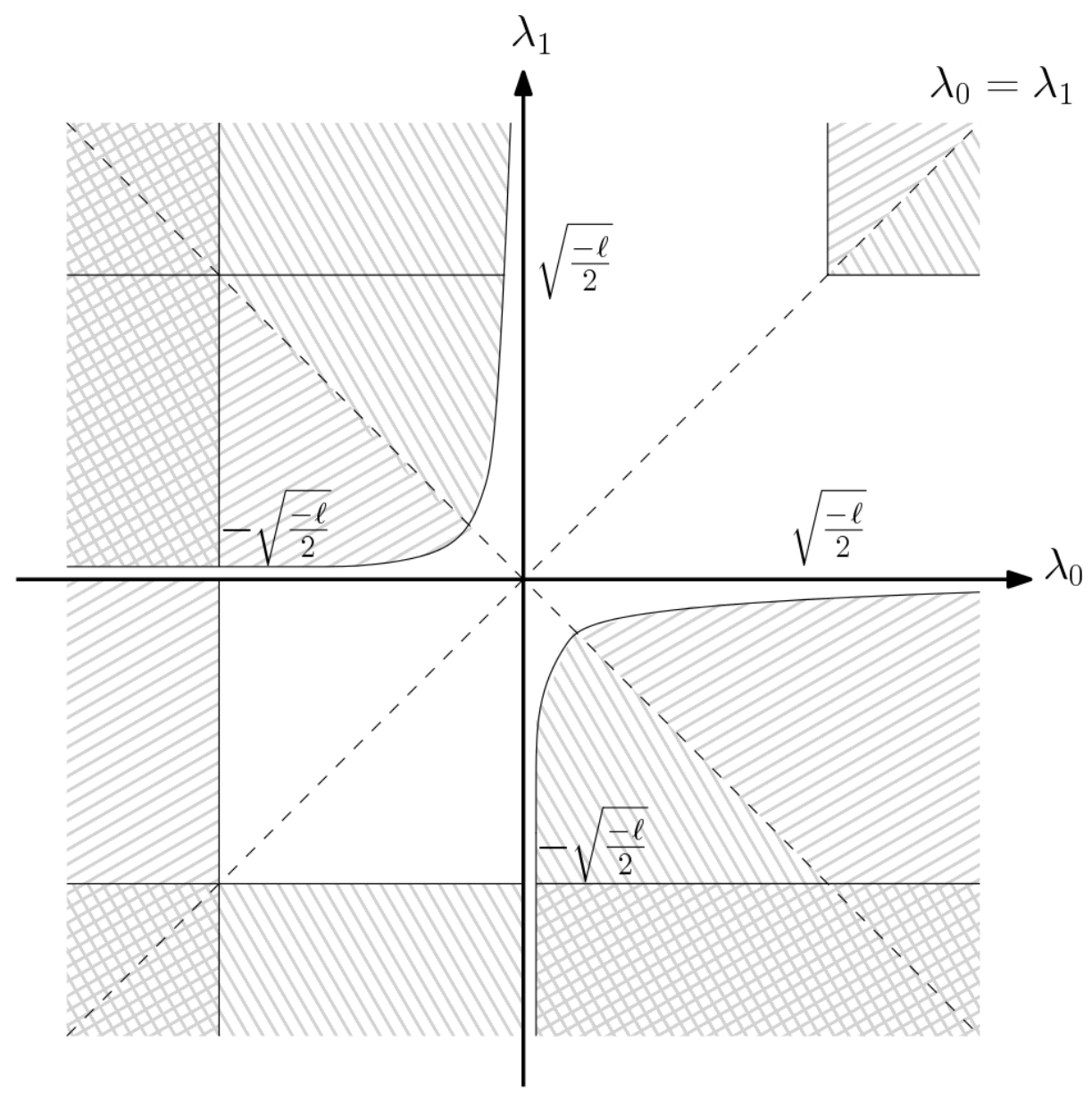

Figure B.2: $F_{\lambda_{\beta}}$.

and hence

$$
\begin{aligned}
F_{\lambda_{\beta}}(\ell)= & \operatorname{Pr}\left\{\lambda_{\beta} \leqslant \ell\right\} \\
= & \operatorname{Pr}\left\{-\lambda_{0}^{2} \leqslant \frac{\ell}{2}, \lambda_{0}<\lambda_{1}\right\}+\operatorname{Pr}\left\{-\lambda_{1}^{2} \leqslant \frac{\ell}{2}, \lambda_{1}<\lambda_{0}\right\} \\
& +\operatorname{Pr}\left\{-\lambda_{0} \leqslant \frac{\ell}{2 \lambda_{1}}, \lambda_{0} \geqslant \lambda_{1}\right\}+\operatorname{Pr}\left\{-\lambda_{1} \leqslant \frac{\ell}{2 \lambda_{0}}, \lambda_{1} \geqslant \lambda_{0}\right\} \\
= & 1-\operatorname{Pr}\left\{\lambda_{0}^{2}>\frac{-\ell}{2}, \lambda_{0}<\lambda_{1}\right\}-\operatorname{Pr}\left\{\lambda_{1}^{2}>\frac{-\ell}{2}, \lambda_{1}<\lambda_{0}\right\} \\
& -\operatorname{Pr}\left\{\lambda_{0}>\frac{-\ell}{2 \lambda_{1}}, \lambda_{0} \geqslant \lambda_{1}\right\}-\operatorname{Pr}\left\{\lambda_{1}>\frac{-\ell}{2 \lambda_{0}}, \lambda_{1} \geqslant \lambda_{0}\right\} .
\end{aligned}
$$

Once again, the shaded areas in Fig. B.2 show the regions satisfying these inequalities 
in (B.22).

Thus

$$
\begin{aligned}
F_{\lambda_{\beta}}(\ell)= & \operatorname{Pr}\left\{0 \leqslant \lambda_{0} \leqslant \infty, 0 \leqslant \lambda_{1} \leqslant \infty\right\} \\
& -\operatorname{Pr}\left\{\sqrt{\frac{-\ell}{2}} \leqslant \lambda_{0} \leqslant \infty, \sqrt{\frac{-\ell}{2}} \leqslant \lambda_{1} \leqslant \infty\right\} \\
& +\operatorname{Pr}\left\{-\sqrt{\frac{-\ell}{2}} \leqslant \lambda_{0} \leqslant 0,-\sqrt{\frac{-\ell}{2}} \leqslant \lambda_{1} \leqslant 0\right\} \\
= & {\left[1-\frac{1}{2}-\frac{1}{2}+\frac{1}{2} \cdot \frac{1}{2}\right] } \\
& -\left[1-F_{\lambda_{0}}\left(\sqrt{\frac{-\ell}{2}}\right)-F_{\lambda_{1}}\left(\sqrt{\frac{-\ell}{2}}\right)+F_{\lambda_{0}}\left(\sqrt{\frac{-\ell}{2}}\right) F_{\lambda_{1}}\left(\sqrt{\frac{-\ell}{2}}\right)\right] \\
& +\left[\frac{1}{2} \cdot \frac{1}{2}-\frac{1}{2} F_{\lambda_{0}}\left(-\sqrt{\frac{-\ell}{2}}\right)-\frac{1}{2} F_{\lambda_{1}}\left(-\sqrt{\frac{-\ell}{2}}\right)+F_{\lambda_{0}}\left(-\sqrt{\frac{-\ell}{2}}\right) F_{\lambda_{1}}\left(-\sqrt{\frac{-\ell}{2}}\right)\right] \\
= & \frac{1}{2}-\left[1-2 F_{\lambda_{0}}\left(\sqrt{\frac{-\ell}{2}}\right)-F_{\lambda_{0}}^{2}\left(\sqrt{\frac{-\ell}{2}}\right)\right]-\left[F_{\lambda_{0}}\left(-\sqrt{\frac{-\ell}{2}}\right)-F_{\lambda_{0}}^{2}\left(-\sqrt{\frac{-\ell}{2}}\right)\right] .
\end{aligned}
$$

For $F_{\lambda_{\gamma}}(\ell),($ B.14) can be written as

$$
\begin{aligned}
\lambda_{\gamma} & =0.25\left[\left|\lambda_{0}-\lambda_{1}\right|-\left|\lambda_{0}+\lambda_{1}\right|\right]^{2} \\
& =0.25 \times \begin{cases}{\left[\lambda_{0}-\lambda_{1}-\lambda_{0}-\lambda_{1}\right]^{2}} & \text { if } \lambda_{0}>\lambda_{1} \\
{\left[\lambda_{0}-\lambda_{1}+\lambda_{0}+\lambda_{1}\right]^{2}} & \text { if } \lambda_{1}<-\lambda_{0} \\
{\left[\lambda_{1}-\lambda_{0}-\lambda_{0}-\lambda_{1}\right]^{2}} & \text { if } \lambda_{1}>\lambda_{0} \\
{\left[\lambda_{1}-\lambda_{0}+\lambda_{0}+\lambda_{1}\right]^{2}} & \text { if } \lambda_{0}<-\lambda_{1}\end{cases} \\
& = \begin{cases}\lambda_{1}^{2} & \text { if } \lambda_{0}>\lambda_{1} \\
\lambda_{0}^{2} & \text { if } \lambda_{1}<-\lambda_{0} \\
\lambda_{0}^{2} & \text { if } \lambda_{1}>\lambda_{0} \\
\lambda_{1}^{2} & \text { if } \lambda_{0}<-\lambda_{1},\end{cases}
\end{aligned}
$$




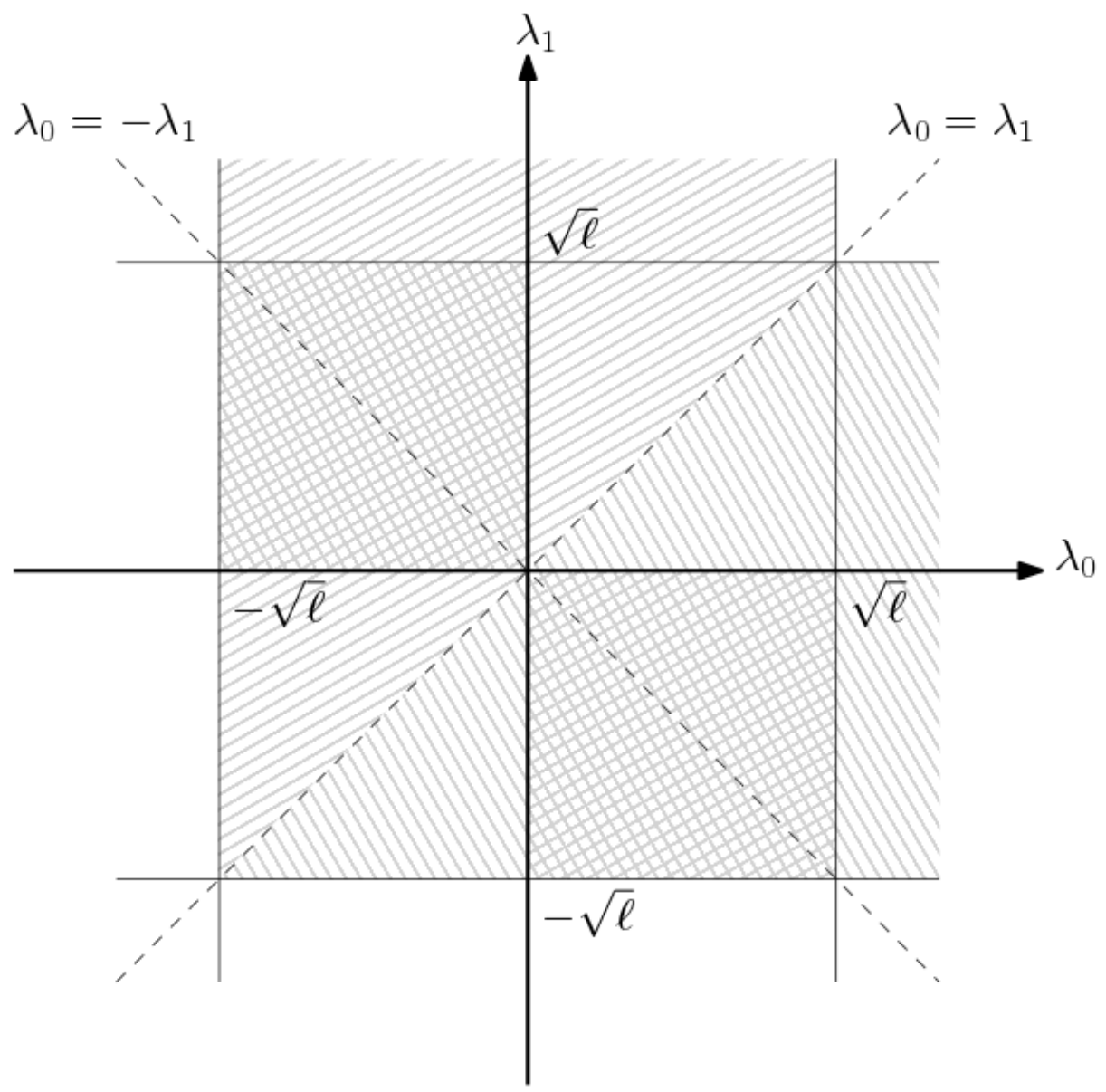

Figure B.3: $F_{\lambda_{\gamma}}$.

then

$$
\begin{aligned}
F_{\lambda_{\gamma}}(\ell)= & \operatorname{Pr}\left\{\lambda_{\gamma} \leqslant \ell\right\} \\
= & \operatorname{Pr}\left\{\lambda_{1}^{2} \leqslant \ell, \lambda_{0}>\lambda_{1}\right\}+\operatorname{Pr}\left\{\lambda_{0}^{2} \leqslant \ell, \lambda_{1}<-\lambda_{0}\right\} \\
& +\operatorname{Pr}\left\{\lambda_{0}^{2} \leqslant \ell, \lambda_{1}>\lambda_{0}\right\}+\operatorname{Pr}\left\{\lambda_{1}^{2} \leqslant \ell, \lambda_{0}<-\lambda_{1}\right\} \\
= & \operatorname{Pr}\left\{-\sqrt{\ell} \leqslant \lambda_{1} \leqslant \sqrt{\ell}, \lambda_{0}>\lambda_{1}\right\}+\operatorname{Pr}\left\{-\sqrt{\ell} \leqslant \lambda_{0} \leqslant \sqrt{\ell}, \lambda_{1}<-\lambda_{0}\right\} \\
& +\operatorname{Pr}\left\{-\sqrt{\ell} \leqslant \lambda_{0} \leqslant \sqrt{\ell}, \lambda_{1}>\lambda_{0}\right\}+\operatorname{Pr}\left\{-\sqrt{\ell} \leqslant \lambda_{1} \leqslant \sqrt{\ell}, \lambda_{0}<-\lambda_{1}\right\} .
\end{aligned}
$$

Again, the shaded areas in Fig. B.3 show the regions satisfying the inequalities in (B.25). 
Therefore

$$
\begin{aligned}
F_{\lambda_{\gamma}}(\ell)= & 1-\operatorname{Pr}\left\{\lambda_{0}>\sqrt{\ell}, \lambda_{1}>\sqrt{\ell}\right\}-\operatorname{Pr}\left\{\lambda_{0} \leqslant-\sqrt{\ell}\right\}-\operatorname{Pr}\left\{\lambda_{1} \leqslant-\sqrt{\ell}\right\} \\
& +\operatorname{Pr}\left\{\lambda_{0} \leqslant-\sqrt{\ell}, \lambda_{1} \leqslant-\sqrt{\ell}\right\} \\
= & F_{\lambda_{0}}(\sqrt{\ell})+F_{\lambda_{1}}(\sqrt{\ell})-F_{\lambda_{0}}(\sqrt{\ell}) F_{\lambda_{1}}(\sqrt{\ell})-F_{\lambda_{0}}(-\sqrt{\ell})-F_{\lambda_{1}}(-\sqrt{\ell}) \\
& +F_{\lambda_{0}}(-\sqrt{\ell}) F_{\lambda_{1}}(-\sqrt{\ell}) \\
= & 2 F_{\lambda_{0}}(\sqrt{\ell})-F_{\lambda_{0}}^{2}(\sqrt{\ell})-2 F_{\lambda_{0}}(-\sqrt{\ell})+F_{\lambda_{0}}^{2}(-\sqrt{\ell})
\end{aligned}
$$

which is the same result as in (B.20).

It is shown in (B.20) and (B.26) that the random variable takes only nonnegative values and takes negative values in (B.23); with the exception that the random variable in the last two terms of (B.22) may accept nonnegative values. To determine the expectation of $\left|\tilde{\lambda}_{2}\right|^{2}$, we substitute those distributions into (B.15) as the following:

$$
\begin{aligned}
\mathrm{E}\left[\left|\tilde{\lambda}_{2}\right|^{2}\right]= & \int_{0}^{\infty}\left[1-\left(2 F_{\lambda_{0}}(\sqrt{\ell})-F_{\lambda_{0}}^{2}(\sqrt{\ell})-2 F_{\lambda_{0}}(-\sqrt{\ell})+F_{\lambda_{0}}^{2}(-\sqrt{\ell})\right)\right] \mathrm{d} \ell \\
& +\int_{0}^{\infty} \frac{1}{2} \mathrm{~d} \ell \quad \begin{array}{c}
\text { due nonnegative random variable in the last two } \\
\text { terms of }(\text { B.22). See Fig. B.4. }
\end{array} \\
& -\int_{-\infty}^{0}\left[\frac{1}{2}-\left(1-2 F_{\lambda_{0}}\left(\sqrt{\frac{-\ell}{2}}\right)-F_{\lambda_{0}}^{2}\left(\sqrt{\frac{-\ell}{2}}\right)\right)-\left(F_{\lambda_{0}}\left(-\sqrt{\frac{-\ell}{2}}\right)-F_{\lambda_{0}}^{2}\left(-\sqrt{\frac{-\ell}{2}}\right)\right)\right] \mathrm{d} \ell \\
= & \int_{0}^{\infty}\left[1-\left(1-2 F_{\lambda_{0}}(\sqrt{-\ell})+F_{\lambda_{0}}^{2}(\sqrt{-\ell})\right)+\left(1-2 F_{\lambda_{0}}(\sqrt{\ell})+F_{\lambda_{0}}^{2}(\sqrt{\ell})\right)\right] \mathrm{d} \ell \\
& +\int_{-\infty}^{0}\left[\left(1-2 F_{\lambda_{0}}\left(\sqrt{\frac{-\ell}{2}}\right)-F_{\lambda_{0}}^{2}\left(\sqrt{\frac{-\ell}{2}}\right)\right)+F_{\lambda_{0}}\left(-\sqrt{\frac{-\ell}{2}}\right)-F_{\lambda_{0}}^{2}\left(-\sqrt{\frac{-\ell}{2}}\right)\right] \mathrm{d} \ell \\
= & \int_{0}^{\infty}\left[1-\left(1-F_{\lambda_{0}}(-\sqrt{\ell})\right)^{2}+\left(1-F_{\lambda_{0}}(\sqrt{\ell})\right)^{2}\right] \mathrm{d} \ell \\
& +\int_{-\infty}^{0}\left[\left(1-F_{\lambda_{0}}\left(\sqrt{\frac{-\ell}{2}}\right)\right)^{2}+F_{\lambda_{0}}\left(-\sqrt{\frac{-\ell}{2}}\right)-F_{\lambda_{0}}^{2}\left(-\sqrt{\frac{-\ell}{2}}\right)\right] \mathrm{d} \ell .
\end{aligned}
$$




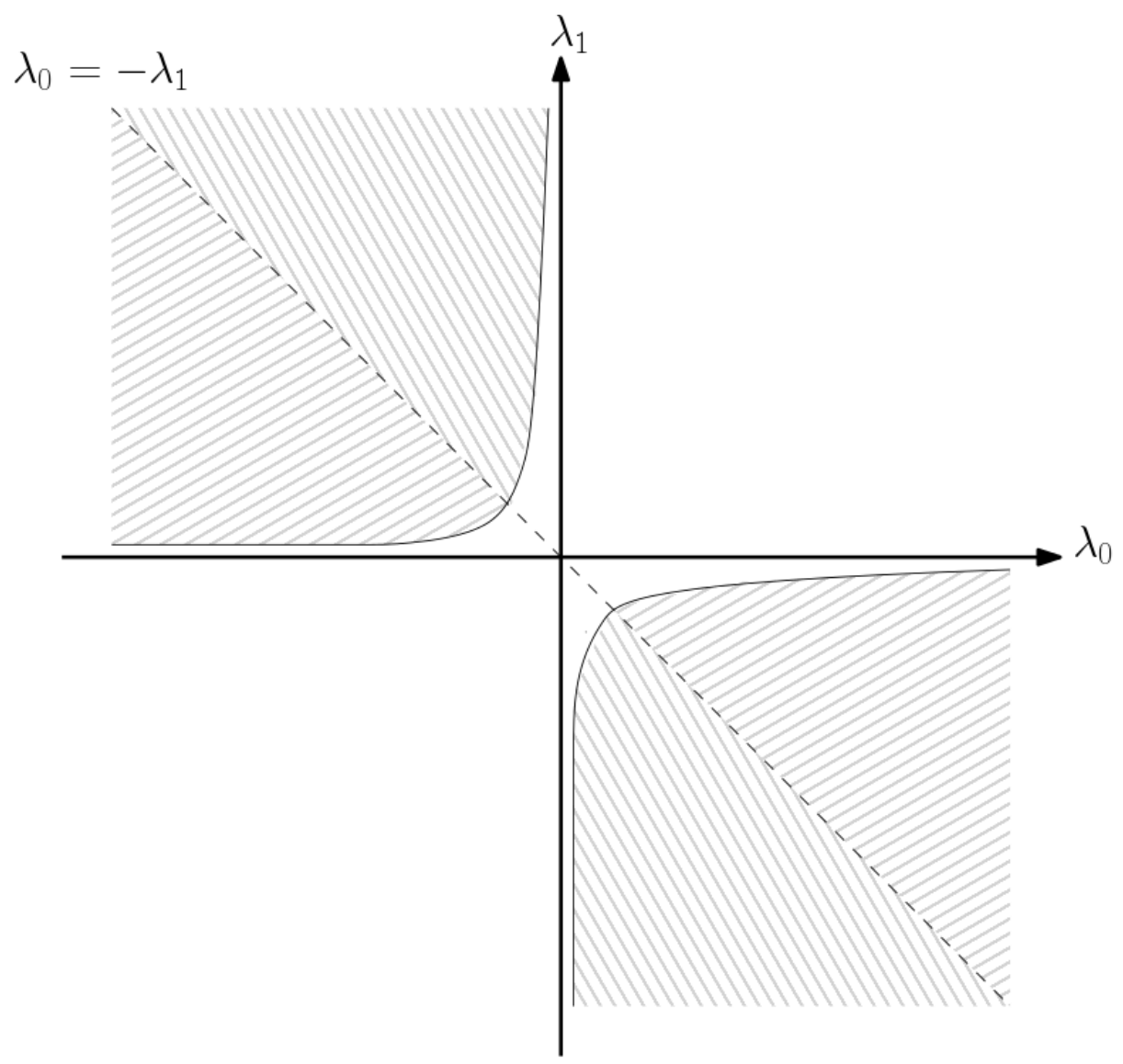

Figure B.4: $\operatorname{Pr}\left\{\lambda_{0}>\frac{-\ell}{2 \lambda_{1}}, \lambda_{0} \geqslant \lambda_{1}\right\}+\operatorname{Pr}\left\{\lambda_{1}>\frac{-\ell}{2 \lambda_{0}}, \lambda_{1} \geqslant \lambda_{0}\right\} \simeq \frac{1}{2}$.

It follows with $F_{\lambda}(\ell)=1-\mathrm{Q}\left(\frac{\ell+\mu_{\lambda}}{\sigma_{\lambda}}\right)-\mathrm{Q}\left(\frac{\ell-\mu_{\lambda}}{\sigma_{\lambda}}\right)$ that 


$$
\begin{aligned}
& \mathrm{E}\left[\left|\tilde{\lambda}_{2}\right|^{2}\right]=\int_{0}^{\infty}[1-\left(\mathrm{Q}\left(\frac{-\sqrt{\ell}+\mu_{\lambda_{0}}}{\sigma_{\lambda_{0}}}\right)+\mathrm{Q}\left(\frac{-\sqrt{\ell}-\mu_{\lambda_{0}}}{\sigma_{\lambda_{0}}}\right)\right)^{2} \\
&+\left.\left(\mathrm{Q}\left(\frac{\sqrt{\ell}+\mu_{\lambda_{0}}}{\sigma_{\lambda_{0}}}\right)+\mathrm{Q}\left(\frac{\sqrt{\ell}-\mu_{\lambda_{0}}}{\sigma_{\lambda_{0}}}\right)\right)^{2}\right] \mathrm{d} \ell \\
&+\int_{-\infty}^{0}\left[\left(\mathrm{Q}\left(\frac{\sqrt{\frac{-\ell}{2}}+\mu_{\lambda_{0}}}{\sigma_{\lambda_{0}}}\right)+\mathrm{Q}\left(\frac{\sqrt{\frac{-\ell}{2}}-\mu_{\lambda_{0}}}{\sigma_{\lambda_{0}}}\right)\right)^{2}\right. \\
&+1-\mathrm{Q}\left(\frac{-\sqrt{\frac{-\ell}{2}}+\mu_{\lambda_{0}}}{\sigma_{\lambda_{0}}}\right)-\mathrm{Q}\left(\frac{-\sqrt{\frac{-\ell}{2}}-\mu_{\lambda_{0}}}{\sigma_{\lambda_{0}}}\right) \\
&\left.-\left(1-\mathrm{Q}\left(\frac{-\sqrt{\frac{-\ell}{2}}+\mu_{\lambda_{0}}}{\sigma_{\lambda_{0}}}\right)-\mathrm{Q}\left(\frac{-\sqrt{\frac{-\ell}{2}}-\mu_{\lambda_{0}}}{\sigma_{\lambda_{0}}}\right)\right)^{2}\right] \mathrm{d} \ell
\end{aligned}
$$

with $\mathrm{Q}(\ell)=1-\mathrm{Q}(-\ell)$. Finally, simplification of (B.28) takes several steps and gives:

$$
\begin{aligned}
\mathrm{E}\left[\left|\tilde{\lambda}_{2}\right|^{2}\right]= & \int_{0}^{\infty} 1-4\left[\mathrm{Q}\left(\frac{\mu_{\lambda_{0}}+\sqrt{\ell}}{\sigma_{\lambda_{0}}}\right)-\mathrm{Q}\left(\frac{\mu_{\lambda_{0}}-\sqrt{\ell}}{\sigma_{\lambda_{0}}}\right)\right] \mathrm{d} \ell \\
& +\int_{-\infty}^{0} 1-3\left[\mathrm{Q}\left(\frac{\mu_{\lambda_{0}}+\sqrt{\frac{-\ell}{2}}}{\sigma_{\lambda_{0}}}\right)-\mathrm{Q}\left(\frac{\mu_{\lambda_{0}}-\sqrt{\frac{-\ell}{2}}}{\sigma_{\lambda_{0}}}\right)\right] \mathrm{d} \ell \\
= & \int_{0}^{\mu_{\lambda_{0}}^{2}} 1-4\left[\mathrm{Q}\left(\frac{\mu_{\lambda_{0}}+\sqrt{\ell}}{\sigma_{\lambda_{0}}}\right)-\mathrm{Q}\left(\frac{\mu_{\lambda_{0}}-\sqrt{\ell}}{\sigma_{\lambda_{0}}}\right)\right] \mathrm{d} \ell \\
& +\int_{-2 \mu_{\lambda_{0}}^{2}}^{0} 1-3\left[\mathrm{Q}\left(\frac{\mu_{\lambda_{0}}+\sqrt{\frac{-\ell}{2}}}{\sigma_{\lambda_{0}}}\right)-\mathrm{Q}\left(\frac{\mu_{\lambda_{0}}-\sqrt{\frac{-\ell}{2}}}{\sigma_{\lambda_{0}}}\right)\right] \mathrm{d} \ell .
\end{aligned}
$$

\section{B.3 Tangent Line Approximation}

In this section we are going to prove equation (3.67). 
Let

$$
y=f(x)
$$

as in Fig. B.5, it is approximately the same as its tangent line

$$
y=f\left(x_{0}\right)+f^{\prime}\left(x_{0}\right)\left[x-x_{0}\right]
$$

The tangent line approximates $f(x)$. It gives a good approximation near the tangent point $x_{0}$. Moving away from $x_{0}$, however, the approximation grows less accurate.

$$
f(x) \approx f\left(x_{0}\right)+f^{\prime}\left(x_{0}\right)\left[x-x_{0}\right] .
$$

As an example, let $f(x)=\ln x$. Then $f^{\prime}(x)=\frac{1}{x}$. Suppose the base point is $x_{0}=1$ because we can easily evaluate $\ln 1=0$. Note also that $f^{\prime}\left(x_{0}\right)=\frac{1}{1}=1$.

Then the formula for linear approximation tells us that:

$$
\begin{aligned}
f(x) & \approx f\left(x_{0}\right)+f^{\prime}\left(x_{0}\right)\left[x-x_{0}\right] \\
\ln x & \approx \ln 1+1[x-1] \\
\ln x & \approx 0+[x-1] \\
\ln x & \approx x-1 .
\end{aligned}
$$

The point of linear approximation is that the curve (in this case $y=\ln x$ ) is approximately the same as the tangent line $(y=x-1)$ when $x$ is close to the base point $x_{0}$. 


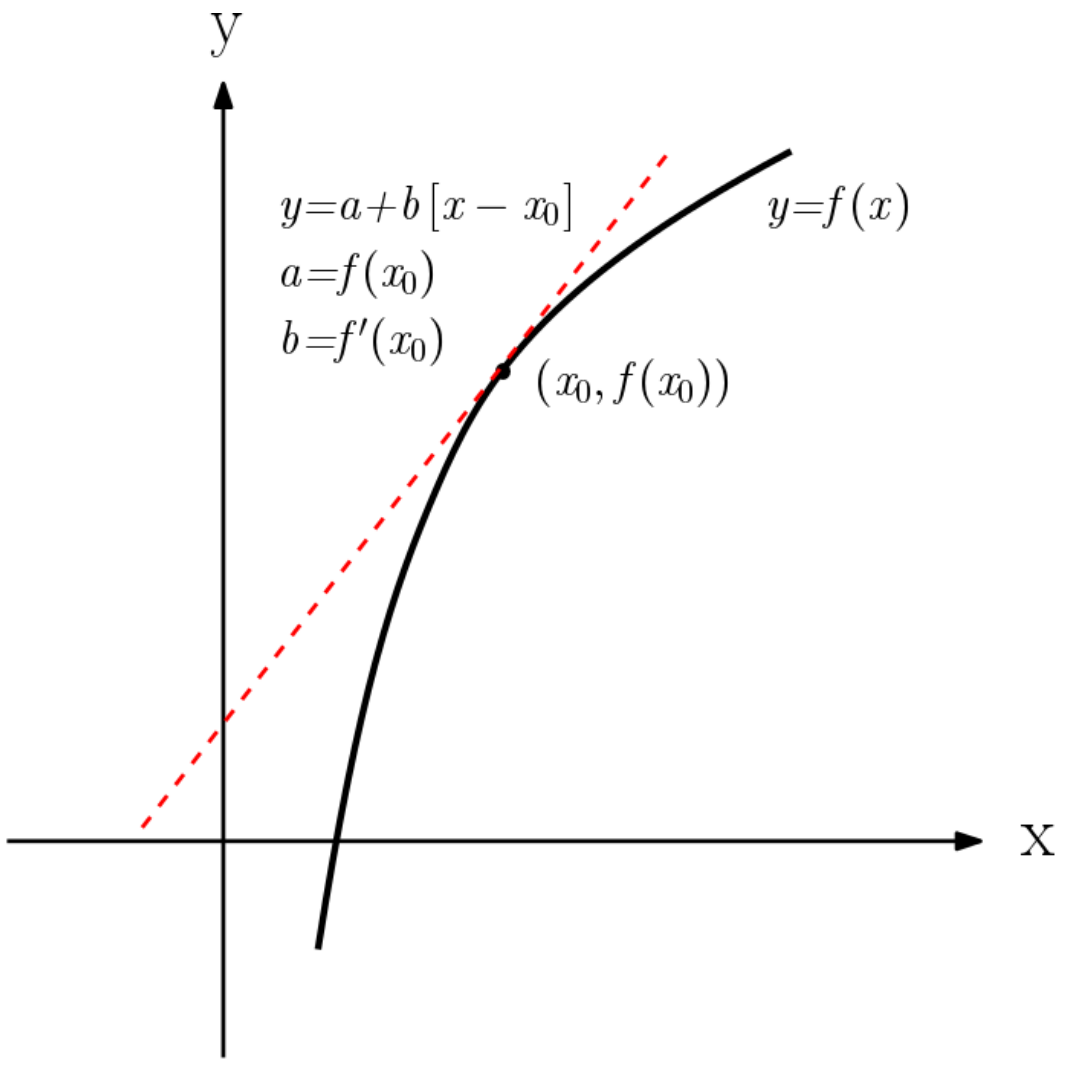

Figure B.5: Tangent as a linear approximation to a curve. 


\section{Appendix $\mathrm{C}$}

\section{Hermite Polynomials $\mathbf{H}_{n}(x)$ : Some Basics}

Hermite's differential equation could be written as

$$
\overline{\bar{y}}-2 x \bar{y}+2 n y=0,
$$

\section{Important Properties [94]}

- Rodrigues' Formula: Hermite polynomials $\mathrm{H}_{n}(x)$ is expressed using Rodrigues formula

$$
\mathrm{H}_{n}(x)=(-1)^{n} e^{\frac{x^{2}}{2}} \frac{\mathrm{d}^{n}}{\mathrm{~d} x^{n}}\left(e^{\frac{-x^{2}}{2}}\right), \quad n=0,1, \cdots
$$

- First Few Hermite Polynomials: The first three Hermite polynomials are

$$
\mathrm{H}_{0}(x)=1, \quad \mathrm{H}_{1}(x)=x, \quad \mathrm{H}_{2}(x)=x^{2}-1, \quad \mathrm{H}_{3}(x)=x^{3}-3 x, \cdots
$$

- Generating Function:

$$
e^{t x-\frac{t^{2}}{2}}=\sum_{n=0}^{\infty} \mathrm{H}_{n}(x) \frac{t^{n}}{n !}
$$

- Orthogonality: For $-\infty<x<\infty$ we have

$$
\int_{-\infty}^{\infty} e^{\frac{x^{2}}{2}} \mathrm{H}_{m}(x) \mathrm{H}_{n}(x) \mathrm{d} x= \begin{cases}0 & \text { if } m \neq n \\ \sqrt{2 \pi} n ! \delta_{m n} & \text { if } m=n .\end{cases}
$$

And a given function $f(x)$ is possible to be express as

$$
f(x)=\sum_{n=0}^{\infty} \mathrm{c}_{n} \mathrm{H}_{n}(x) \quad-\infty<x<\infty
$$


where

$$
\mathrm{c}_{n}=\frac{1}{\sqrt{2 \pi} n !} \int_{-\infty}^{\infty} e^{\frac{x^{2}}{2}} f(x) \mathrm{H}_{n}(x) \mathrm{d} x .
$$

- Even/Odd Functions: According to $\mathrm{H}_{n}(-x)=(-1)^{n} \mathrm{H}_{n}(x)$, we have

- $\mathrm{H}_{n}(x)$ is an even function when $n$ is even.

- $\mathrm{H}_{n}(x)$ is an odd function when $n$ is odd.

- Expected Values: For a random variable $x$ with $\mu$ is its expected value, 1 is its variance, and has a Gaussian distribution then

$$
\mathrm{E}\left[\mathrm{H}_{n}(x)\right]=\mu^{n}
$$

And the moment is

$$
\begin{aligned}
\mathrm{E}\left[x^{2 n}\right] & =(-1)^{n} \mathrm{H}_{2 n}(0) \\
& =(2 n-1) ! !
\end{aligned}
$$

where

$$
\begin{aligned}
& n ! !=\prod_{k=0}^{m}(n-2 k)=n(n-2)(n-4) \cdots \text { with } 0 ! !=1 \\
& m=\left\lceil\frac{n}{2}\right\rceil-1, \text { and } \\
& \mathrm{H}_{n}(0)= \begin{cases}0 & \text { when } n \text { is odd } \\
(-1)^{\frac{n}{2}} & \text { otherwise. }\end{cases}
\end{aligned}
$$

